UCRL-LR-123744

Distribution Category UC-910

\title{
Saturation of Langmuir Waves in \\ Laser-Produced Plasmas
}

\author{
Kevin Louis Baker
}

(Ph.D. Thesis)

Manuscript date: April 1996

LAWRENCE LIVERMORE NATIONAL LABORATORY

University of California • Livermore, California • 94551

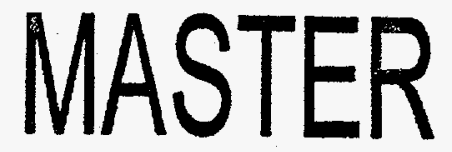




\section{DISCLAIMIER}

Portions of this document may be illegible in electronic image products. Images are produced from the best available original document. 


\title{
Saturation of Langmuir Waves in Laser-Produced Plasmas
}

\author{
By \\ Kevin Louis Baker \\ B.S. (Mississippi State University) 1989 \\ M.S. (University of California, Davis) 1991 \\ DISSERTATION
}

Submitted in partial satisfaction of the requirements for the degree of

DOCTOR OF PHILOSOPHY

in

Applied Science

in the

GRADUATE DIVISION

of the

UNIVERSITY OF CALIFORNIA

DAVIS

Approved:

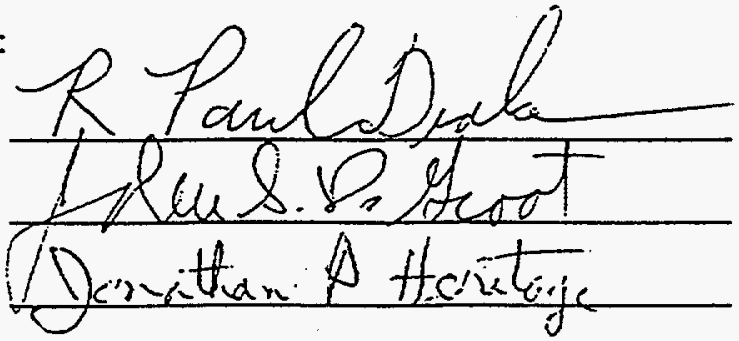

Committee in Charge

1996 


\section{Contents}

Acknowledgments

vi

List of Figures

viii

Abstract

$x v$

1 Introduction

1.1 Introduction 1

1.2 Plasma Basics 4

1.3 Parametric Instabilities $\quad 7$

$\begin{array}{lll}1.4 & \text { Layout of the Dissertation } & 14\end{array}$

2 Saturation Mechanisms $\quad 17$

2.1 Introduction 17

2.2 Saturation from Damping Effects 17

2.3 Saturation due to Plasma Inhomogeneities 19

2.4 Pump Depletion 29

2.5 Secondary Decay Processes 32

2.6 Modulational Instability and Collapse of the Langmuir $\begin{array}{ll}\text { Waves } & 37\end{array}$

2.7 Ponderomotive Detuning 43

2.8 Mode Coupling(Unstimulated Processes) 48 
a. Self-interaction Between Different Decay Triangles

b. Interaction Between Different Instabilities

2.9 Relativistic Detuning

2.10 Particle Trapping and Wave Breaking

3 Electromagnetic Decay Instability

3.1 Introduction

3.2 Electromagnetic Decay Instability from Two Plasmon Decay

3.3 Seeding of Simulated Brillouin Scattering and Saturation of the Ion Acoustic Decay Instability

3.4 Electromagnetic Decay Instability from Stimulated Raman Scattering

3.5 Summary

3.6 Future Work

4 Thomson Scattering

4.1 Introduction

4.2 Thomson Scattering in Homogeneous Plasmas

4.3 Thomson Scattering in Inhomogeneous Plasmas

5 Initial Thomson Scattering Experiments; Verification of Raman Scattering Models

5.1 Introduction 
5.2 Experimental Description $\quad 124$

5.3 Collective Thomson Scattering 129

$\begin{array}{lll}5.4 & \text { Plasma Characterization } & 131\end{array}$

5.5 Experimental Observations 136

$\begin{array}{lll}5.6 & \text { Discussion } & 140\end{array}$

5.7 Summary 143

6 Thomson Scattering Measurements of the Langmuir Wave Spectra Resulting from Stimulated Raman Scattering 146

$\begin{array}{lll}6.1 & \text { Introduction } & 146\end{array}$

$\begin{array}{lll}6.2 & \text { Experimental Layout } \quad 148\end{array}$

6.3 Plasma Characterization 154

6.4 Experimental Results 165

$\begin{array}{lll}6.5 & \text { Discussion } & 170\end{array}$

$\begin{array}{lll}6.6 & \text { Summary } & 174\end{array}$

$\begin{array}{lll}7 & \text { Summary } & 175\end{array}$

Appendix 2.1 Derivation of Equations Describing Stimulated Raman Scattering

Appendix 2.2 Rosenbluth Model for Parametric Instabilities in $\begin{array}{ll}\text { Inhomogeneous Plasmas } & 187\end{array}$

Appendix 2.3 K-space Resonance Width for Steady State 192

Appendix 2.4 Numerical Solution of Rosenbluth Equations 197 
Appendix 2.5 Saturation of Forward Raman Scattering Due to Ponderomotive Detuning

Appendix 2.6 Saturation of Forward Raman Scattering Due to Relativistic Detuning

Appendix 3.1 Numerical calculation of the scattering version of EDI from an inhomogeneous plasma

Appendix 4.1 Derivation of the Thomson Scattered Signal Using the Fluid Equations

Appendix 4.2 Derivation of the Thomson Scattering Form Factor in a Homogeneous Plasma

Appendix 4.3 Numerical Calculation of Thomson Scattering from an Inhomogeneous Plasma

Appendix 4.4 Graphical Solution of the Roots of the Dispersion Relation Corresponding to Ion Acoustic Waves

Appendix 4.5 Contour Integrations for Thomson Scattering 


\section{Acknowledgments}

I would first like to thank my dissertation advisor Paul Drake for suggesting the saturation of Langmuir waves as my dissertation topic. His support and guidance during my time as a graduate student is very much appreciated. I would like to thank my academic advisor John De Groot for first instilling in me an interest in plasma physics which set the foundation for my graduate work.

I would like to thank Kent Estabrook for his contributions, which included all of the LASNEX simulations of the experiments conducted for this dissertation. In addition, I had numerous discussions with him regarding the material presented in this dissertation. I would like to thank him most, however, for his support and encouragement throughout the course of this dissertation. I would also like to thank David Villeneuve for the time he spent teaching me a number of the experimental skills that I would use throughout the work presented in this dissertation. I would also like to thank him for providing me with experimental time on the LP2 laser system, as well as for a number of subsequent discussions. I thank Russ Evans for his help with the LabView programming used with one of the diagnostics. I would also like to thank Christine Labaune and Hector Baldis for the time they allowed me on the LULI laser system.

Over the course of my dissertation, I have had the opportunity to work with and learn from a number of people collaborating on experiments. These collaborators include: David Villeneuve, and Bruno Lafontaine from the 
National Research Council; Paul Drake, Keith Bradley, Bruno Bauer, Katsu Mizuno, Steven Batha, and Brad Sleaford from the Plasma Physics Research Institute; Sophie Baton, Nathalie Renard, Christine Labaune, Elisa Schifano, and Hector Baldis from Ecole Polytechnique; Wolf Seka and Ray Bahr from the Laboratory for laser Energetics; and Bob Watt from the Los Alamos National Laboratory.

During my graduate studies, I have profited from a number of discussions on topics related to my dissertation. In particular, I would like to thank Bedros Afeyan for countless discussions on parametric instabilities. I also had a great many discussions with Sasha Rubenchik which primarily focused on strong turbulence issues. My discussions with Linda Powers regarding numerical simulations were very beneficial in the development of most of the FORTRAN codes listed in the appendices. I had a number of conversations with Ed Williams on a wide range of topics, the most notable of which guided the development of the code used to solve for the roots of the plasma dispersion relation in multi-ion plasmas. I would also like to thank Denise Hinkel for her discussions concerning resonance absorption. I also profited from discussions with Martin Goldman, Tudor Johnston, Wojtek Rozmus, and Don DuBois.

I would like to express my appreciation to my parents and sisters for their moral support throughout my graduate experience, as well as the many preceding years. I would like to thank Christine Coverdale, my closest friend in graduate school, for her support and encouragement. I would also like to thank a number of my closest friends, Tony Correa, Russ Evans, and Christine Shannon, who provided an outlet for me over the years. 


\section{List of Figures}

1.3.1 Physical description of parametric instabilities illustrating how the wave interactions lead to instability.

1.3.2 Graph showing the corona region of a laser produced plasma.

1.3.3 Contour plots of the dispersion relation for stimulated Raman scattering.

2.2.1 Homogeneous damping stabilization of convective parametric instabilities.

2.3.1 Convective versus absolute parametric instabilities in laserproduced plasmas.

2.3.2 Numerical solution of the Rosenbluth equations for a linear phase mismatch between the interacting waves.

2.3.3 Measured amplification of the scattered electromagnetic wave associated with stimulated Raman forward scattering . . . . . .

2.5.1 Figure showing the amplitude of the Langmuir wave at the threshold for convective instability. 
2.6.1 Range of unstable ion wavenumbers, $\mathrm{kia}_{\mathrm{i}}$, driven by the modulational instability.

2.7.1 Comparison of the Langmuir wave amplitude at saturation predicted by the ponderomotive detuning model(dashed . . . .

2.8.1 Wavenumber resolved Thomson scatter spectrum from Langmuir waves and ion waves participating in . . . . . . .

2.10.1 Approximate Langmuir wave amplitude for which two plasmon decay goes below the convective damping . . . . . . .

2.10.2 Plot showing the amplitude at which wave breaking becomes important for Langmuir waves.

2.10.3 Flux of stimulated Raman scattering driven from a gold solid target.

3.2.1 Plot of the $\omega_{0} / 2$ emission observed from a solid target experiment.

3.2.2 Plot of the absolute magnitude of $3 \omega_{0} / 2$ emission and $\omega_{0} / 2$ emission as a function of incident laser intensity. 
3.2.3 Schematic representation of inverse resonance absorption in an inhomogeneous plasma.

3.3.1 Wavevector diagram showing the coupling process which leads to electromagnetic emission near the plasma frequency.

3.4.1 Finite spatial pump effects on the absolute and convective nature of parametric instabilities.

3.4.2 Schematic representation of absolute electromagnetic decay driven by stimulated Raman scattering.

4.2.1 Thomson scattering form factor for a $50: 50 \mathrm{CH}$ plasma at various values of $T_{i} / T_{e}$.

4.2.2 Dependence of the shape factor on the ratio of ion to electron temperature for a $\mathrm{CH}$ plasma.

4.2.3 Attributes of the Thomson scattering shape factor, $S(k, \omega)$, for a $\mathrm{CH}$ plasma.

4.2.4 Thomson scatter spectra in a $\mathrm{CH}$ plasma as a function of $\mathrm{k} \lambda_{\mathrm{De}}$ with $\mathrm{T}_{\mathrm{i}} / \mathrm{T}_{\mathrm{e}}$ fixed at 0.5 . 
4.2.5 Thomson shape factor for a Carbon plasma in which there exists a finite drift between the electrons and ions.

4.3.1 Numerical evaluation of the Thomson scattered vector potential for the case of a linear phase mismatch between . . . .

5.1.1 Model distribution of the electrons in a plasma containing a hot electron distribution.

5.2.1 Experimental layout showing the interaction and probe beams, along with the interaction chamber.

5.2.2 Schematic of the detector circuit used for the Au:Ge detector.

5.2.3 Signal attenuation as a function of round trip passes through the image dissector.

5.4.1 LASNEX model for the incident laser. The FWHM of the laser .

5.4.2 LASNEX prediction for the maximum electron density averaged over a $200 \mu \mathrm{m}$ radius cylinder as a function of . . . . .

5.4.3 LASNEX estimation for the electron temperature averaged over a $200 \mu \mathrm{m}$ radius cylinder as a function of time for $\mathrm{a}$. . . . 
5.4.4 Ion wave damping as a function of the ratio of ion temperature, $T_{i}$, to electron temperature, $T_{e}$, for a Collodium ..

5.5.1 Thomson scattering and stimulated Raman scattering observed from a $0.35 \mu \mathrm{m}$ Collodium thin foil.

5.5.2 Composite graph showing the Thomson scattered signal from the Langmuir waves driven in six different shots.

5.5.3 Figure 5.5.3 Range of unstable ion wavenumbers, $k_{i a}$, driven by the modulational instability. The horizontal axis represents . .

5.6.1 The $3 / 2 \omega_{0}$ emission obtained from a solid Carbon target which maintained a critical surface throughout the duration of . . . .

6.2.1 Experimental setup showing the Thomson scattering diagnostic, as well as the stimulated Raman Scattering . . . . .

6.2.2 Schematic of one of the detector circuits used for the Germanium diode array.

6.2.3 Oscilloscope trace showing the gate signal, lower trace, which was sent to the 2249 A charge integrator and the diode . . . . . . 
6.3.1 LASNEX predicted evolution of the maximum of the plasma density as a function of time.

6.3.2 LASNEX prediction for time evolution of the electron and ion temperatures present in the plasma.

6.3.3 Spectrum from the heater beam stimulated Raman scattering diagnostic.

6.3.4 Concept behind the use of stimulated Raman scattering as a temperature diagnostic.

6.3.5 Corresponding temperature of the plasma such that a Langmuir wave with a given $k \lambda_{\text {De product participates in } \mathrm{a} . \ldots} 162$

6.3.6 Thomson scattering spectrum taken with an interaction beam intensity of approximately $5 \times 10^{12} \mathrm{~W} / \mathrm{cm}^{2}$.

6.4.1 Thomson scattering measurement of the Langmuir wave spectrum using a f/3.3 probe beam (a) and the backscattered . . . 166

6.4.2 Thomson scattering measurement of the Langmuir wave spectrum using a $f / 25$ probe beam. 
A.2.4.1 Grid spacing used in the numerical program in the case of backscatter where the group velocity of the two waves are . . . 201

A.4.3.1 Thomson scattered vector potential produced in a plasma with a quadratic phase mismatch between the interacting . . 235

A.4.4.1 Graphical solution of the roots of the dispersion relation in a Carbon plasma.

A.4.4.2 Graphical solution of the roots of the dispersion relation in a Collodium plasma.

A.4.4.3 Graphical solution of the roots of the dispersion relation in a $\mathrm{CH}$ plasma.

A.4.4.4 Absolute magnitude of the ion wave damping normalized to the real part of the ion acoustic frequency for four different .

A.4.5.1 Contour used to evaluate $\int \exp \left(\mathrm{im}^{2}\right) \mathrm{dm}$.

A.4.5.2 Contours used to evaluate $\int \exp \left(\mathrm{im}^{3}\right) \mathrm{dm}$. 
Kevin Louis Baker

March 1996

Applied Science

\title{
Saturation of Langmuir Waves in Laser-Produced Plasmas
}

\begin{abstract}
This dissertation deals with the interaction of an intense laser with a plasma (a quasineutral collection of electrons and ions). During this interaction, the laser drives large-amplitude waves through a class of processes known as parametric instabilities. Several such instabilities drive one type of wave, the Langmuir wave, which involves oscillations of the electrons relative to the nearly-stationary ions. There are a number of mechanisms which limit the amplitude to which Langmuir waves grow. In this dissertation, these mechanisms are examined to identify qualitative features which might be observed in experiments and/or simulations. In addition, a number of experiments are proposed to specifically look for particular saturation mechanisms.

In a plasma, a Langmuir wave can decay into an electromagnetic wave and an ion wave. This parametric instability is proposed as a source for electromagnetic emission near half of the incident laser frequency observed from laser-produced plasmas. This interpretation is shown to be consistent with existing experimental data and it is found that one of the previous
\end{abstract}


mechanisms used to explain such emission is not. The scattering version of the electromagnetic decay instability is shown to provide an enhanced noise source of electromagnetic waves near the frequency of the incident laser.

In the experiments performed for this dissertation, a probe laser was scattered from the electrons present in the plasma to measure the spectrum of Langmuir waves driven in the plasma. This technique, known as Thomson scattering, was used to identify the mechanisms producing the Langmuir waves, as well as to identify mechanisms responsible for the saturation of these Langmuir waves. The Thomson-scattering measurements indicated that the Langmuir-wave spectrum resulted from stimulated Raman scattering, which is the decay of the laser into an electromagnetic wave and a Langmuir wave. In a related experiment, these measurements detected Langmuir waves which were driven by the Langmuir decay instability, which is the decay of a Langmuir wave into a second Langmuir wave and an ion acoustic wave. This measurement represents the first observation of the three-wave parametric instability known as the Langmuir decay instability in laser-produced plasmas. 


\section{Chapter 1}

\section{Introduction}

\subsection{Introduction}

This dissertation deals with the interaction of an intense laser with a plasma, which can loosely be called ionized matter. During this interaction, the laser can drive large amplitude waves in the plasma through a class of processes known as parametric instabilities. These are discussed in greater detail in section 1.3. This dissertation examines the mechanisms which limit the amplitude to which these waves can be driven by the incident laser. This work is primarily motivated by the saturation of Langmuir waves, which are also known as electron plasma waves. Consequently, the experimental work described below used a parametric instability known as stimulated Raman scattering to generate the Langmuir waves used to study these saturation mechanisms. Stimulated Raman scattering is the decay of an electromagnetic wave (the laser) into a scattered electromagnetic wave and an electron plasma wave or Langmuir wave. 
Raman scattering occurs in a number of plasma applications which include inertial confinement fusion, particle acceleration, current-drive in Tokamaks, and X-ray-laser plasmas, to name a few. In some of these applications, such as particle acceleration and current-drive in Tokamaks, Raman scattering is beneficial and it is desirable to drive large amplitude Langmuir waves to increase efficiency. In other applications such as inertial confinement fusion and $x$-ray lasers, stimulated Raman scattering is detrimental and it is important to limit the amplitude that the Langmuir waves are driven. It is therefore important to understand the saturation mechanisms which limit the growth of these parametric instabilities as a first step in the eventual process of controlling the amplitude to which these processes grow.

As stated above, in inertial confinement fusion and $X$-ray-laser plasmas, stimulated Raman scattering is a detrimental process. In inertial confinement fusion, lasers are used to compress deuterium and tritium targets to very high densities. Parametric instabilities which drive electromagnetic waves take energy away from the incident laser, thereby reducing the efficiency at which the energy is coupled into the target. In addition, parametric instabilities which drive Langmuir waves can lead to the generation of distributions of electrons with very high temperatures. These hot electrons can preheat the fuel in inertially confined plasmas which increases the energy required to compress the fuel to the necessary densities. In the case of $x$-ray laser schemes, stimulated Compton scattering can cause a heating of the background electron distribution resulting in a loss of efficiency[1]. Understanding the saturation mechanisms which limit the 
amplitude of the waves driven in these instabilities may lead to more efficient ways to control the growth of these instabilities.

As mentioned in the first paragraph, there are also several applications in which Raman scattering is beneficial. The Langmuir wave driven in Raman scattering can be used for particle acceleration[2-4]. In this case, a Langmuir wave with a phase velocity close to the speed of light is used to accelerate electrons to very high energy. The electrons traveling slightly slower than the wave see a nearly constant potential in the wave frame which allows them to be efficiently accelerated. Raman scattering can also be used for current drive in Tokamaks[5-8]. In this case Raman scattering is used to drive a Langmuir wave with a phase velocity several times the thermal velocity of electrons in a plasma. In this application near thermal electrons in the plasma are accelerated by the Langmuir wave in a preferential direction, generating a net current around the Tokamak. All of these applications are limited by the amplitude to which the Langmuir wave can be driven and an understanding of these applications requires detailed knowledge of the saturation mechanisms responsible for limiting the amplitude of these waves.

Secondary parametric instabilities can also be beneficial in many circumstances. In laser-produced plasmas, an instability can saturate when one of its daughter waves becomes large enough that it can become the pump wave for a secondary parametric instability[9-13]. Therefore, although the original parametric instability is detrimental, the secondary parametric instability is beneficial because it limits the amplitude that the original 
parametric instability can grow. This process is discussed in further detail in section 2.5 of chapter 2 .

The second section of this chapter reviews some of the basic principles of plasmas. This section also discusses the normal modes present in the plasma which make up the waves driven by parametric instabilities. The third section of chapter 1 introduces parametric instabilities and reviews the physical mechanism responsible for the growth of parametric instabilities. This section also gives a brief description of the parametric instabilities discussed in the rest of the dissertation along with the location where these instabilities occur. Finally, the fourth section of chapter 1 gives a brief outline of the subsequent chapters contained in this dissertation.

\subsection{Plasma Basics}

A plasma can be defined as an ionized gas consisting of electrons and ions in which the kinetic energy of its constituent particles is much greater than their potential energy[14,15]. Using the above definition for a plasma, it has been estimated that more than $99 \%$ of the universe is in the plasma state. Applications involving plasmas include the generation of fusion energy, thin-film deposition, manufacturing of microelectronics, particle accelerators, isotope separation, gas lasers, fluorescent bulbs, and gas treatment to name a few.

An important property of a plasma is Debye shielding. As a test charge is placed in a plasma the particles arrange themselves to screen out the potential formed by the test charge. The potential of a test particle at rest is 
given by $\Phi=\frac{q}{r} \exp \left(-\frac{r}{\lambda_{D e}}\right)[14,15]$. The Debye length, $\lambda_{\mathrm{De}}=\mathrm{vth} / \omega_{\mathrm{pe}}$, is then the distance over which the potential from the test charge has decreased by one exponentiation where $v_{t h}=\left(\kappa T_{e} / m_{e}\right)^{0.5}$ is the thermal velocity of the electrons and $\omega_{\mathrm{pe}}$ is the plasma frequency defined as $\omega_{\mathrm{pe}}=\left(4 \pi \mathrm{q}^{2} \mathrm{n}_{\mathrm{oe}} / \mathrm{m}_{\mathrm{e}}\right)^{0.5}$. The definition given for a plasma above can then be understood in terms of the number of particles located in a sphere of radius equal to the Debye length. The potential energy, $q \Phi$, being much less than the kinetic energy is easily expressed as $q \Phi=\frac{q^{2}}{r}<<\frac{1}{2} m v_{t h}^{2}$. where the inter-particle spacing, $r$, is approximately equal to the cube root of the electron density, $r \approx n_{\mathrm{oe}}^{-1 / 3}$. The above conditions imply that $1<<\frac{4}{3} \pi \frac{\mathrm{v}_{\mathrm{th}}^{3}}{\omega_{\mathrm{pe}}^{3}} \mathrm{n}_{\mathrm{oe}}=\Lambda$ where $\Lambda$ is the number of particles within a sphere of radius the Debye length, $\lambda_{\mathrm{De}}[14,15]$. The definition of a plasma given above then simply requires that the number of particles within the shielding cloud must be very large. This definition excludes strongly-coupled plasmas in which the number of particles within the Debye sphere is very small such as occurs in the overdense partially ionized region of solid target laser-plasma experiments and also in the relatively cold plasmas formed by short-pulse high-intensity lasers. A more inclusive definition of a plasma might simply be ionized matter.

Another important property of plasmas used throughout this dissertation is the plasma frequency, $\omega$ pe, defined above. The plasma frequency is the resonant frequency which the electrons in the plasma respond. If the ions in the plasma were displaced slightly from the electrons, the two slabs representing the electrons and ions, would oscillate like a 
harmonic oscillator. This oscillation frequency, $\omega$, would approximately be at the plasma frequency, $\omega^{2}=\omega_{\mathrm{pe}}^{2}+\omega_{\mathrm{pi}}^{2}$, where $\omega_{\mathrm{pi}}=\left(4 \pi \mathrm{q}^{2} \mathrm{n}_{\mathrm{oi}} / \mathrm{m}_{\mathrm{i}}\right)^{0.5}$ and $\omega_{\mathrm{pi}} \ll \omega_{\mathrm{pe}}$

In a homogeneous plasma with no externally imposed electromagnetic or magnetic fields, there are only three normal modes present; electromagnetic waves, electrostatic ion waves, and Langmuir waves, also known as electron plasma waves. These modes satisfy the following dispersion relations in a field free plasma:

$$
\begin{array}{ll}
\omega_{\mathrm{o}}^{2}=\omega_{\mathrm{pe}}^{2}+\mathrm{c}^{2} \mathrm{k}_{\mathrm{o}}^{2} & \text { for electromagnetic waves, } \\
\omega_{\mathrm{epw}}^{2}=\omega_{\mathrm{pe}}^{2}+3 \mathrm{v}_{\mathrm{th}}^{2} \mathrm{k}_{\mathrm{epw}}^{2} & \text { for Langmuir waves, and } \\
\omega_{\mathrm{ia}}-\overrightarrow{\mathrm{k}}_{\mathrm{ia}} \cdot \overrightarrow{\mathrm{u}}_{\mathrm{f}}=\mathrm{c}_{\mathrm{s}} \mathrm{k}_{\mathrm{ia}} & \text { for ion acoustic waves, }
\end{array}
$$

which are the more-common, long-wavelength regime of electrostatic ion waves. Here $\omega_{o}\left(k_{0}\right), \omega_{e p w}\left(k_{e p w}\right), \omega_{i a}\left(k_{i a}\right)$ are the frequency(wavenumber) of the electromagnetic wave, the Langmuir wave, and the ion acoustic wave, respectively, and $c$ is the speed of light, $v$ th is the electron thermal velocity, $c_{S}$ is the ion sound speed, and uf is the flow velocity of the plasma. Both the Langmuir waves and the ion acoustic waves are electrostatic waves with their electric fields parallel to their wavevectors while the electromagnetic waves are transverse waves with their electric field perpendicular to their wavevector. The Langmuir waves and the electromagnetic waves are high frequency waves whose frequency must be greater than the plasma frequency. 
This is simply due to the fact that the electrons in the plasma react on time scales dictated by the plasma frequency. When a Langmuir wave or electromagnetic wave has a frequency less than the plasma frequency, the electrons in the plasma react faster than the wave can push them around which results in a net damping of the Langmuir or electromagnetic wave. The point at which the plasma frequency is equal to the frequency of the electromagnetic wave, therefore defines the classical turning point for a normally incident electromagnetic wave. The density corresponding to this turning point is known as the critical density, $\mathrm{n}_{\mathrm{Cr}}$, and is defined as $n_{c r}=\left(m_{e} \omega_{0}^{2}\right)\left(4 \pi q^{2}\right)^{-1}$. The ion acoustic wave on the other hand has a frequency which is much less than the plasma frequency. The ion acoustic wave obeys the same dispersion relation as an acoustic wave in air, provided $\mathrm{k}_{\mathrm{ia}} \lambda_{\mathrm{De}}<<1$. The motion of acoustic waves in air result from the gradient in pressure between regions of compression and rarefaction. The motion of ion acoustic waves in plasmas result from both the gradient in ion pressure between the regions of rarefaction and compression and from a build up of charge in these regions due to the pressure of the electrons present in the plasma.

\subsection{Parametric Instabilities}

This dissertation deals primarily with parametric instabilities driven when a high-intensity laser interacts with a plasma. In this context, the laser intensity, $\mathrm{I}$, is high when $\mathrm{I}>10^{13} \mathrm{~W} / \mathrm{cm}^{2}$. A parametric instability occurs when there exists a wave in the plasma which has sufficient amplitude that it can in 
turn drive other waves in the plasma unstable. In this case, the wave driving the instability is known as the pump wave while the waves being driven are referred to as the daughter waves. Parametric instabilities present in laserproduced plasmas are often driven by the electromagnetic wave associated with the high-intensity laser incident on the plasma.

One example of a parametric instability clriven in laser-produced plasmas is stimulated Raman scattering. This represents the decay of an electromagnetic wave into a scattered electromagnetic wave and an electron plasma wave[16,17]. This instability is analogous to stimulated Raman scattering driven in nonlinear optical media where the incident electromagnetic wave scatters from quantized electronic, vibrational, molecular, or optical lattice phonon modes[18]. The mechanism of instability in the case of stimulated Raman scattering in laser-produced plasmas is illustrated in figure 1.3.1[19]. The oscillation velocity of the electrons, $u_{o s}$, in the plasma associated with the incident electromagnetic wave couples with a density fluctuation, $\delta n_{e p w}$, present in the plasma. This coupling produces a transverse scattered current, $j_{s}=q \delta n_{e p w} u_{o s}$, which acts as a source term to drive a scattered electromagnetic wave, of electric field $E_{s}$. The scattered electromagnetic wave beats with the incident electromagnetic wave resulting in a "ponderomotive force", pushing plasma out of regions of high field pressure, which in turn enhances the original density fluctuation. This process forms a closed loop leading to instability.

The term, "ponderomotive force" deserves a bit more explanation: The plasma tries to maintain a pressure balance between the particle pressure and the wave pressure. Therefore, regions of high wave-pressure push the plasma 
particles out so as to maintain a pressure balance. The corresponding "force", which is proportional to the gradient in the time-averaged pressure associated with high-frequency waves, is known as the ponderomotive force. This force may be produced by either electromagnetic or Langmuir waves, and may involve the pressure of a given wave or the low-frequency pressure associated with the beating of two waves. This ponderomotive force is responsible for pushing the plasma from regions of high field.
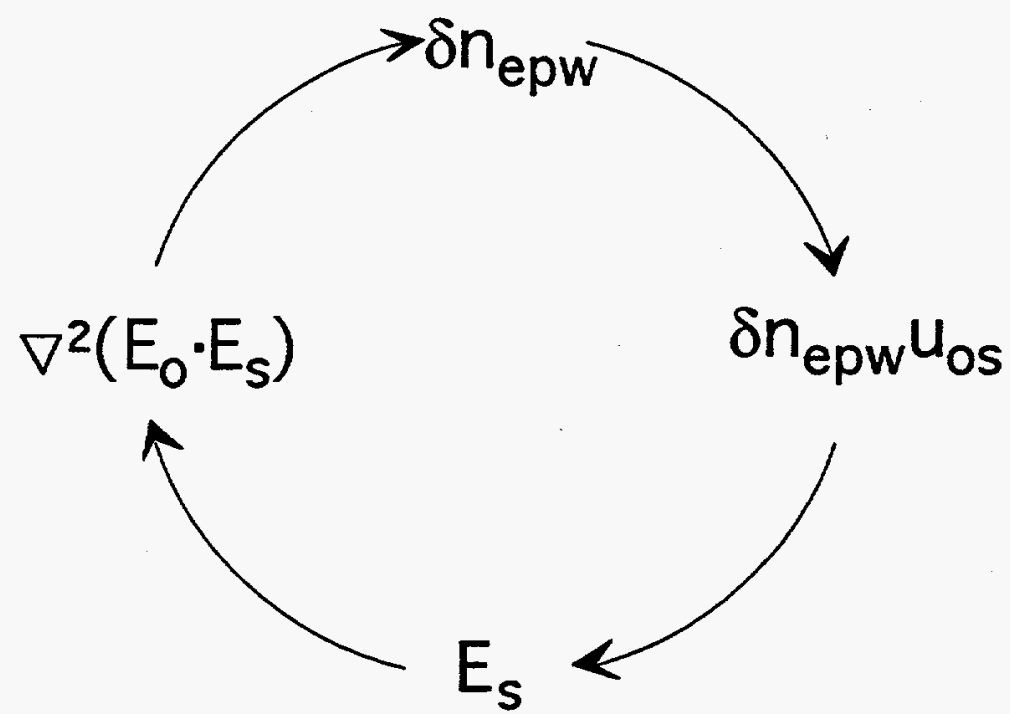

Figure 1.3.1 Physical description of parametric instabilities illustrating how the wave interactions lead to instability.

Parametric instabilities obey the frequency and wavenumber matching conditions,

$$
\begin{aligned}
& \omega_{\mathrm{o}}=\omega_{\mathrm{s}}+\omega_{\mathrm{epw}} \text { and } \\
& \overrightarrow{\mathrm{k}}_{\mathrm{o}}=\overrightarrow{\mathrm{k}}_{\mathrm{s}}+\overrightarrow{\mathrm{k}}_{\mathrm{epw}},
\end{aligned}
$$


where $\omega_{o}, \vec{k}_{0} ; \omega_{s}, \vec{k}_{s} ;$ and $\omega_{\text {epw }}, \vec{k}_{\text {epw }}$ are the frequency and wavenumber of the incident electromagnetic wave, the scattered electromagnetic wave, and the electron plasma wave respectively. These are the conditions required for a resonant interaction which, in the case of stimulated Raman scattering, allows the ponderomotive force associated with the beating of the two electromagnetic waves to remain in phase with the Langmuir wave density fluctuation such that the waves can interact over a long distance.

The parametric instabilities in which the pump wave is represented by the incident laser are shown in figure 1.3.2. The incident laser travels up to the reflection point at the critical density, $\mathrm{n}_{\mathrm{cr}}$. The parametric instabilities detailed in figure 1.3.2 take place at and below the critical surface in the plasma corona.

The oscillating two stream instability, which involves the decay of the incident electromagnetic wave into an electron plasma wave and a zerofrequency ion wave, occurs primarily near the critical surface. From the critical surface to approximately eighty percent of the critical surface, the incident laser can drive the ion acoustic decay instability. This instability involves the decay of the incident laser into an electron plasma wave and an ion acoustic wave. The low density cutoff for the ion acoustic decay instability occurs when the damping on the Langmuir wave becomes large enough that the instability goes below threshold for instability as discussed in section 2.2. From the quarter critical surface to approximately twenty percent of the critical surface, the incident laser drives two plasmon decay which is the decay of the electromagnetic wave into two Langmuir waves. Again the low density 
cutoff of this instability occurs when the damping on the two Langmuir waves becomes greater than the growth rate for the instability. Below the quarter critical surface, the incident laser drives stimulated Raman scattering which, as mentioned above, is the decay of the incident laser into an electromagnetic wave and a Langmuir wave(at the lowest densities stimulated Raman scattering evolves into stimulated Compton

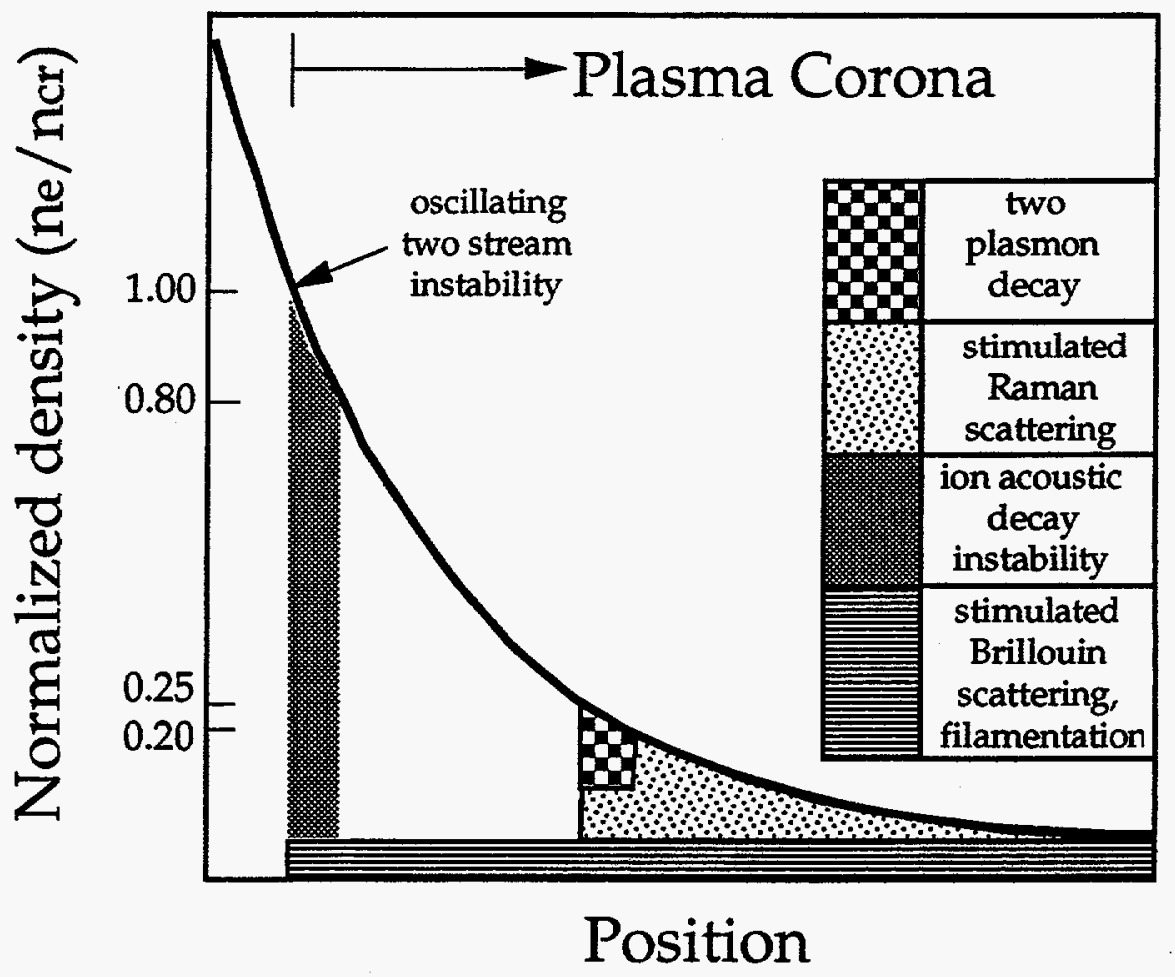

Figure 1.3.2 Graph showing the coronal region of a laser produced plasma. The vertical axis shows the density normalized to the critical density, the reflection point of normally incident light, while the horizontal axis shows the spatial region in front of the target. The parametric instabilities are then labeled showing the density region where each may occur. 
scattering)[20]. All throughout the plasma corona, the laser can drive stimulated Brillouin scattering and filamentation. Stimulated Brillouin scattering is the decay of the incident laser into an ion wave and a scattered electromagnetic wave and filamentation is the forward scattering version of stimulated Brillouin scattering.

As discussed above, stimulated Raman scattering is a parametric instability in which an incident electromagnetic wave drives a scattered electromagnetic wave and a Langmuir wave, also known as an electron plasma wave. The actual derivation of the equations describing stimulated Raman scattering is located in Appendix 2.1. In this Appendix, the equations describing stimulated Raman scattering are Fourier transformed, leading to the dispersion relation for stimulated Raman scattering.

Neglecting higher order harmonics, the dispersion relation describing stimulated Raman scattering is given by

$$
\begin{aligned}
\frac{\omega\left(\omega+\mathrm{i} v_{\mathrm{e}}\right)}{\omega_{\mathrm{pe}}^{2}}-1-3 \mathrm{k}^{2} \lambda_{\mathrm{De}}^{2} & =\frac{\mathrm{k}^{2} \mathrm{v}_{\mathrm{os}}^{2}}{4 \omega_{\mathrm{pe}}^{2}}\left[\frac{1}{\mathrm{D}\left(\omega-\omega_{0}, \overrightarrow{\mathrm{k}}-\overrightarrow{\mathrm{k}}_{\mathrm{o}}\right)}+\right. \\
& \left.\frac{1}{\mathrm{D}\left(\omega+\omega_{0}, \overrightarrow{\mathrm{k}}+\overrightarrow{\mathrm{k}}_{\mathrm{o}}\right)}\right]
\end{aligned}
$$

where $\mathrm{D}(\omega, \overrightarrow{\mathrm{k}})=\omega\left(\omega+\mathrm{i} v_{\mathrm{s}}\right) / \omega_{\mathrm{pe}}^{2}-1-c^{2} \mathrm{k}^{2} / \omega_{\mathrm{pe}}^{2}$. Figure 1.3.3 a and $\mathrm{b}$ are contour plots in which the dashed lines in the contour plot show the locations where the real part of the above dispersion relation is equal to zero and the solid lines show the locations where the imaginary part of the above dispersion relation is equal to zero. In figure 1.3.3 a, the damping is assumed to be zero 
and the remaining parameters are chosen such that the downscattered wave is a resonant mode, $\operatorname{Re}\left[\mathrm{D}\left(\omega-\omega_{0}, \overrightarrow{\mathrm{k}}-\overrightarrow{\mathrm{k}}_{\mathrm{o}}\right)\right]=0$. The intersection points between the real zero contours, dashed lines, and the imaginary zero contours, solid lines, represent some of the roots of the above sixth order dispersion relation. Figure 1.3.3 a shows that there exists one root which is unstable characterized by a positive imaginary component of the frequency.
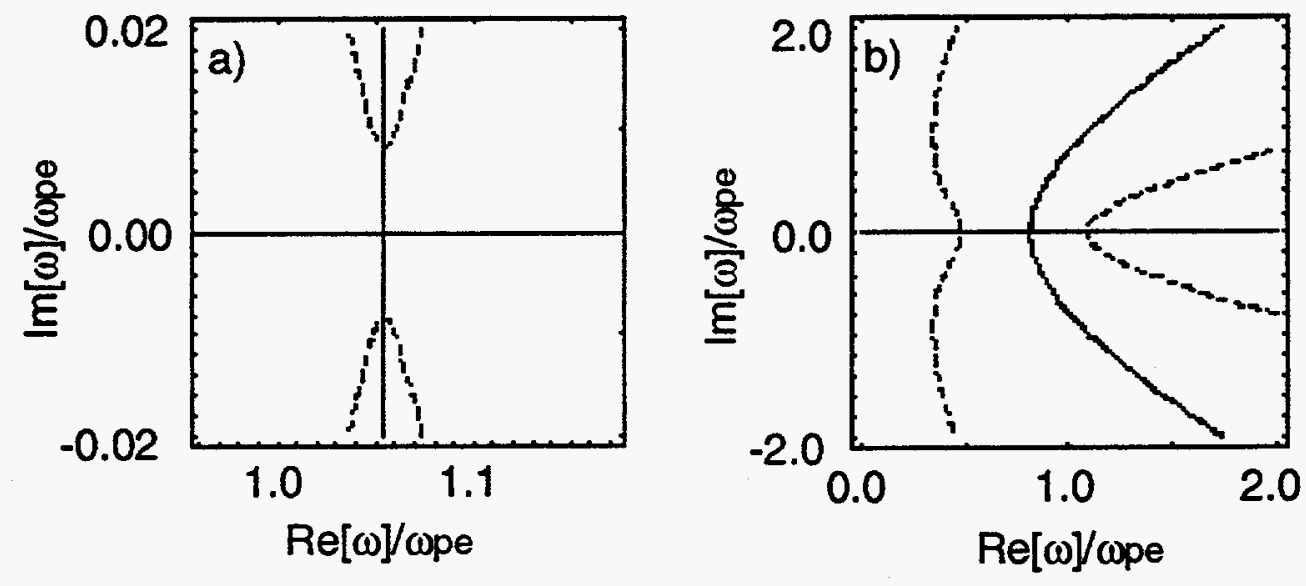

Figure 1.3.3 Contour plots of the dispersion relation for stimulated Raman scattering. Both the vertical and horizontal axes represent frequency normalized to the plasma frequency.

Although the dispersion relation shown in equation 1.3.1 contains upscattered terms in addition to downscattered terms, parametric instabilities are only unstable to the downscattering processes. This can be seen by looking at the zero contours shown in figure 1.3.3 b. In figure 1.3.3 $\mathrm{b}$ the parameters of equation 1 are chosen so that the upscattered component is a resonant mode, $\operatorname{Re}\left[\mathrm{D}\left(\omega+\omega_{0}, \overrightarrow{\mathrm{k}}+\overrightarrow{\mathrm{k}}_{\mathrm{o}}\right)\right] \approx 0$. The dispersion relation in this case is stable as 
characterized by the lack of a root containing a positive imaginary component.

\subsection{Layout of the dissertation}

The second chapter reviews a number of saturation mechanisms and attempts to give simple models to explain how these processes lead to saturation. After the introduction of some of these mechanisms, a section describing relevant experimental evidence for these saturation processes is given. This chapter also tries to address when each of these saturation mechanisms might become important. This chapter also proposes a number of experiments to look for the effects of particular parametric instabilities.

The third chapter considers the role which the electromagnetic decay instability(EDI) plays in laser-produced plasmas. This instability represents the decay of an electron plasma wave into an electromagnetic wave and an ion acoustic wave. The electromagnetic decay instability provides another channel, in addition to Langmuir decay through which parametric instabilities involving Langmuir waves can saturate. As a specific example, the conditions for which EDI is an absolute instability are found for the case where this instability is pumped by the Langmuir wave associated with stimulated Raman scattering. When EDI is pumped by the Langmuir waves associated with two plasmon decay, EDI presents an explanation for $\omega_{0} / 2$ emission from laser-produced plasmas which is consistent with experimental observations. In addition, the scattering of Langmuir waves off of ion acoustic waves near the critical surface is shown to provide an enhanced noise source 
from which stimulated Brillouin scattering can grow. In addition, this enhanced noise source can then appear as an apparent Brillouin signal.

The theory of Thomson scattering is reviewed in Chapter 4. Thomson scattering was the primary diagnostic used in the experiments performed for this dissertation which are described in chapters 5 and 6. Chapter 4 discusses the plasma parameters which can, in principle, be obtained through the use of this diagnostic. Section 4.2 discusses the homogeneous theory of Thomson scattering and presents calculated spectra which are used to infer various properties in the plasma. Section 4.3 looks at the inhomogeneous theory of Thomson scattering and proposes a diagnostic to measure the local flow gradient in a plasma.

The first experiment is then detailed in the fifth chapter. In this experiment Thomson scattering was used to look directly at the Langmuir wave spectrum associated with stimulated Raman scattering. These measurements provided a direct comparison between stimulated Raman scattering and a model known as "enhanced Thomson scattering"[21] as explanations for the electromagnetic emission between $\omega_{0}$ and $\omega_{0} / 2$ observed in laser-produced plasmas. The Thomson scattering measurements showed that the Langmuir wave spectrum resulted from stimulated Raman scattering and not from enhanced Thomson scattering.

The sixth chapter describes an experiment which was designed to look for the Langmuir decay instability. This experiment also used Thomson scattering to measure the Langmuir wave spectrum driven by stimulated Raman scattering. This experiment detected Langmuir waves with components both parallel and antiparallel to the incident laser's wavevector. 
Langmuir waves traveling parallel to the incident laser wavevector were attributed to stimulated Raman scattering, however, the Langmuir waves moving antiparallel to the incident laser, which cannot be explained by stimulated Raman scattering, were attributed to the Langmuir decay instability.

Finally chapter 7 presents a summary the work presented in chapters 2 through 6. Chapter 7 attempts to organize the material presented in the previous chapters. The Appendices contain the calculations discussed in the relevant chapters. 


\section{Chapter 2}

\section{Saturation Mechanisms}

\subsection{Introduction}

In this chapter, a number of saturation mechanisms are introduced and when appropriate simple models are given to explain how these mechanisms lead to saturation. In some cases a section describing relevant experimental evidence for these saturation processes is given. The text of this chapter will be concerned with using simple models to describe these saturation mechanisms with more accurate calculations being placed in the Appendices. This dissertation, and consequently this chapter, is primarily concerned with those saturation mechanisms which act specifically on Langmuir waves.

\subsection{Saturation from damping effects}

This section reviews the effects of damping on parametric instabilities. As discussed in chapter 1, stimulated Raman scattering is a parametric instability in which an incident electromagnetic wave drives a scattered 
electromagnetic wave and a Langmuir wave, also known as an electron plasma wave. The actual derivation of the equations describing stimulated Raman scattering is located in Appendix 2.1. It is necessary to look at the dispersion relation for the waves participating in stimulated Raman scattering to see the effect of damping on the instability. The dispersion relation for stimulated Raman scattering can be found by Fourier transforming the equations for this parametric process which are derived in Appendix 2.1, equations 2.1.6 and 2.1.10.

The dispersion relation for stimulated Raman scattering is given by

$$
\begin{aligned}
\frac{\omega\left(\omega+\mathrm{i} 2 v_{\mathrm{e}}\right)}{\omega_{\mathrm{pe}}^{2}}-1-3 \mathrm{k}^{2} \lambda_{\mathrm{De}}^{2}= & \frac{\mathrm{k}^{2} \mathrm{v}_{\mathrm{os}}^{2}}{4 \omega_{\mathrm{pe}}^{2}}\left[\frac{1}{\mathrm{D}\left(\omega-\omega_{\mathrm{o}}, \overrightarrow{\mathrm{k}}-\overrightarrow{\mathrm{k}}_{\mathrm{o}}\right)}+\right. \\
& \left.\frac{1}{\mathrm{D}\left(\omega+\omega_{\mathrm{o}}, \overrightarrow{\mathrm{k}}+\overrightarrow{\mathrm{k}}_{\mathrm{o}}\right)}\right]
\end{aligned}
$$

where $D(\omega, \vec{k})=\left\{\omega\left(\omega+i 2 v_{s}\right)-\omega_{p e}^{2}-c^{2} k^{2}\right\} \omega_{p e}^{-2}$. Figure 2.2.1a is a contour plot showing the zero contours of the real and imaginary parts of the dispersion relation. In this case the damping is assumed to be zero, and the remaining parameters are chosen such that the downscattered wave is a resonant mode, $\operatorname{Re}\left[D\left(\omega-\omega_{0}, \vec{k}-\vec{k}_{0}\right)\right]=0$. The intersection points between the real zero contours(dashed lines) and the imaginary zero contours(solid lines) represent some of the roots of the above sixth order dispersion relation. The plot shows that there exists one root which is unstable characterized by a positive imaginary component of the frequency. Figure 2.2.1b shows the identical case as figure 2.2.1a except that the product of the damping on the two daughter 
waves is now approximately equal to the square of the growth rate, $\gamma_{0}{ }^{2}=v_{s} v_{e}$. In this case the parametric instability is stable, characterized by a negative imaginary component of the frequency.
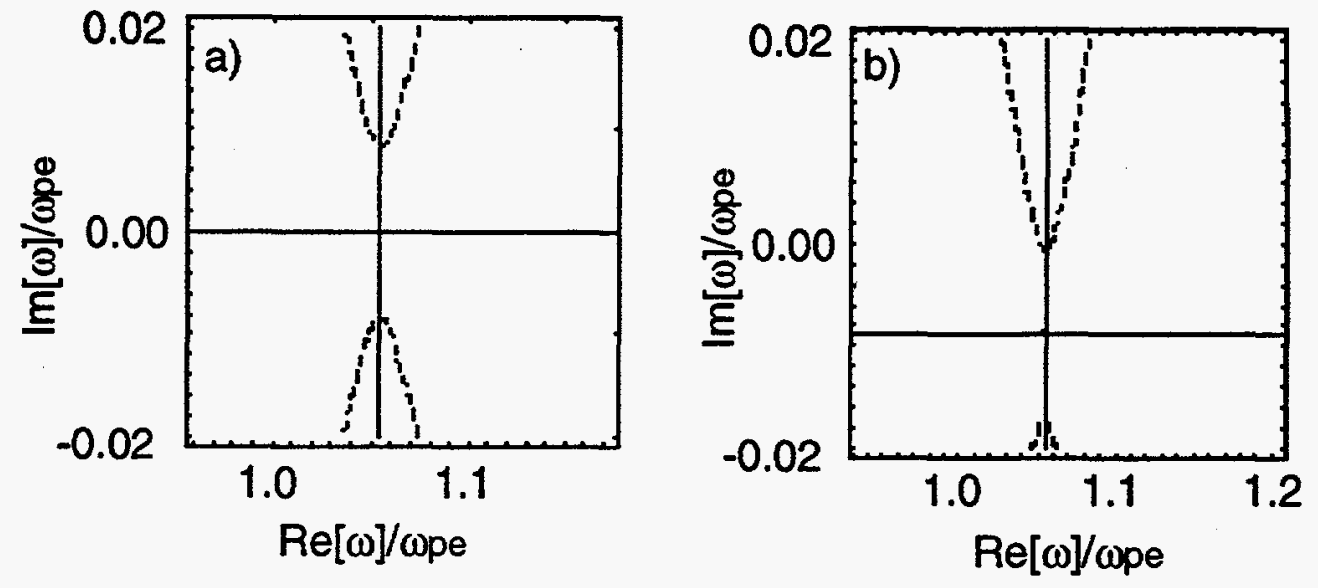

Figure 2.2.1 Homogeneous damping stabilization of convective parametric instabilities.

There have been several examples of damping stabilization of stimulated Raman scattering in laser-produced plasmas[22-24]. In these cases the combination of high $\mathrm{Z}$ and high density combine to increase the collisional damping on both daughter waves. In addition, several of the saturation processes discussed below can lead to saturation of parametric instabilities due to the nonlinear damping caused by the particular saturation mechanism. These processes are discussed for example in section 2.5, 2.8, and 2.10 below.

\subsection{Saturation due to plasma Inhomogeneities}


This section deals with saturation of parametric instabilities due to plasma inhomogeneities. Parametric instabilities can be convective or absolute in nature. Convective instabilities result in a finite gain at any spatial location in the plasma. Absolute instabilities on the other hand continue to grow at a given spatial location until the daughter wave amplitudes become large enough to saturate by some mechanism other than the initial density inhomogeneity itself, although growth of the instability might cause changes to the background density leading to saturation. A graphical representation of the above definitions is shown in figure 2.3.1. In this example the daughter waves have opposing but equal group velocities. Figure 2.3.1 was generated using the numerical code shown in Appendix 2.4. A delta function noise source was used in this example as assumed by Nicholson and Rosenbluth $[7,25]$. There are several conditions which must be satisfied for parametric instabilities to be driven absolute. The group velocity of the daughter waves, along the direction of plasma inhomogeneity, must be opposed. If the group velocity, along the plasma inhomogeneity, of the two daughter waves is in the same direction, then the instability will be convective since eventually both waves will pass any given spatial location producing only a finite gain[26]. Likewise, Rosenbluth showed that a linear phase mismatch between the three interacting waves produced a convective instability, except in the case of two plasmon decay, even when the group velocity of the two daughter waves travel in the opposite direction, this is the case in figure 2.3.1a[27]. Exceptions to this occur when the interaction beam is localized over a short distance or when one of the daughter waves travels perpendicular to the plasma inhomogeneity, in which case absolute 
instability is possible[28]. Plasma inhomogeneities include density, plasma flow, and temperature gradients. Density gradients effect electron plasma waves while plasma flow and temperature gradients primarily effect ion acoustic waves. In laser plasma interactions, plasma flow typically results in a linear phase mismatch between the waves participating in parametric instabilities.
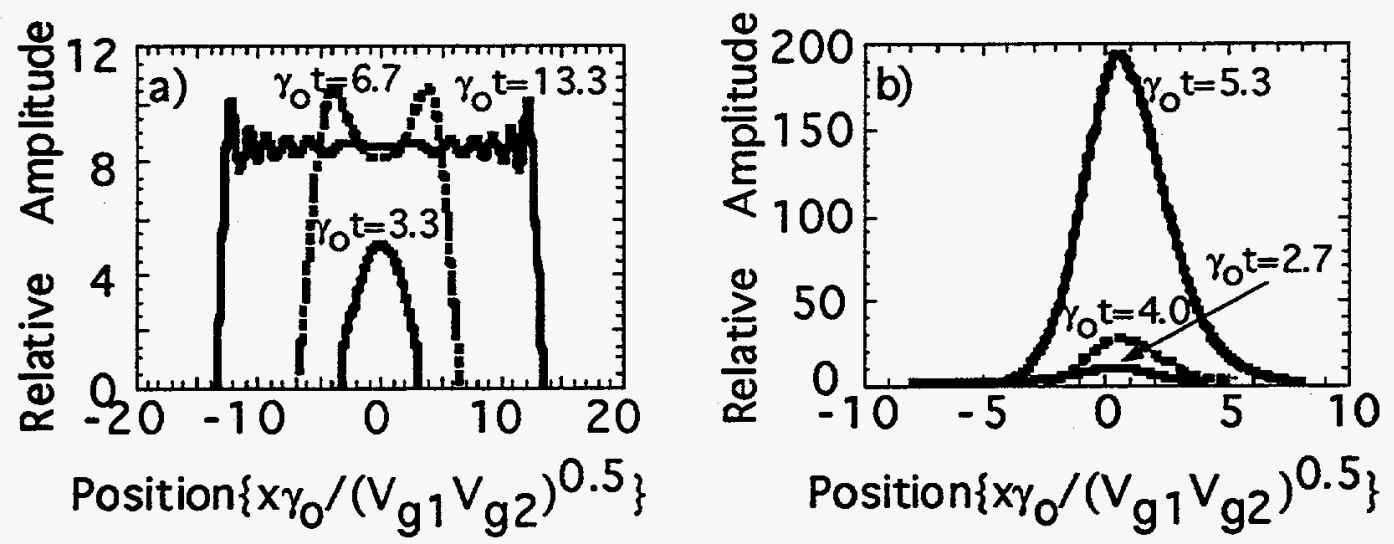

Figure 2.3.1 Convective vs. absolute parametric instabilities in laser-produced plasmas. The convective instability(a) arises from a linear phase mismatch between the interacting waves. In this example the two waves have opposing but equal velocity causing a symmetrical spreading about the perfect phase matching point. The absolute instability(b) arises from a parabolic phase mismatch between the interacting waves. In this case, the two daughter waves again have opposing but equal group velocities. In $b$, however, the quadratic phase mismatch cannot saturate the daughter wave amplitudes and the instability continues to grow.

The derivation of the equations describing stimulated Raman scattering are shown in Appendix 2.1 and follow closely from the derivation 
shown in Kruer 1988[16]. These equations represent the growth of the daughter Langmuir wave and electromagnetic wave participating in stimulated Raman scattering. Rosenbluth showed in 1972 that plasma inhomogeneities can cause these instabilities to have finite gain[27]. The formal derivation of this gain for linear and quadratic phase mismatches is shown in Appendix 2.2.

The parameter dependence obtained by Rosenbluth can be obtained by a qualitative analysis at the homogeneous equations describing the excitation of parametric instabilities in plasmas along with the dispersion relations for the waves in inhomogeneous plasmas. The steady-state homogeneous coupled equations for stimulated Raman scattering without damping,

$$
\begin{aligned}
& \mathrm{V}_{\mathrm{gepw}} \frac{\mathrm{d} \Psi_{\mathrm{epw}}}{\mathrm{dx}}=\gamma_{1} \Psi_{\mathrm{s}}^{*} \text { and } \\
& \mathrm{V}_{\mathrm{gs}} \frac{\mathrm{d} \Psi_{\mathrm{s}}^{*}}{\mathrm{dx}}=\gamma_{2} \Psi_{\mathrm{epw}},
\end{aligned}
$$

are easily solved upon inspection to yield the wave's homogeneous spatial growth rate, $\Psi_{\mathrm{s}}^{*} \alpha \exp \left[\gamma_{\mathrm{o}} \mathrm{x}\left(\mathrm{V}_{\mathrm{gepw}} \mathrm{V}_{\mathrm{gs}}\right)^{-0.5}\right]$, where $\gamma_{\mathrm{o}}=\sqrt{\gamma_{2} \gamma_{1}}$ is the homogeneous growth rate. The saturation level for convective instabilities can be obtained by treating the instability like a driven harmonic oscillator. A harmonic oscillator is only driven efficiently for frequencies which fall within a narrow resonance width. The wavenumber resonance width for parametric instabilities can be found by Fourier transforming the homogeneous equations, 2.3 .1 and 2.3.2, and looking for the full-width at 
half-maximum(FWHM) in wavenumber space. This is detailed in Appendix 2.3 including damping on both daughter waves. Using this prescription, the wavenumber resonance width, neglecting damping, for parametric instabilities is $\Delta \mathrm{k} \propto \gamma_{\mathrm{o}}\left(\mathrm{V}_{\mathrm{gepw}} \mathrm{V}_{\mathrm{gs}}\right)^{-0.5}$. Therefore, the instability is only driven efficiently as long as the wavenumber mismatch between the waves remains within this resonance width.

The length over which the instability can grow is determined by plasma inhomogeneities which dictate how fast the waves become detuned. The wavenumber mismatch,

$$
\begin{aligned}
& \Delta k(x)=\left[k_{o}(x)-k_{s}(x)-k_{e}(x)\right] \approx \\
& \Delta k\left(x_{0}\right)+\left.\left(x-x_{0}\right) \kappa^{\prime}\right|_{x_{0}}+\left.0.5\left(x-x_{0}\right)^{2} \kappa^{\prime \prime}\right|_{x_{0}}
\end{aligned}
$$

can be Taylor expanded about the perfect phase matching point, $x_{0}$, to show how the phase changes with distance[27]. The interaction length, $x-x_{0}$, is found by equating the wavenumber resonance width to the Taylor expanded wavenumber mismatch, $\left.\gamma_{0}\left(V_{\text {gepw }} V_{g s}\right)^{-0.5} \propto\left(x-x_{0}\right) \kappa^{\prime}\right|_{x_{0}}+\left.0.5\left(x-x_{0}\right)^{2} \kappa^{\prime \prime}\right|_{x_{0}}$. The interaction length, $x-x_{0}$, is then given as $x_{\text {int }}=x-x_{0}=\left(-\kappa^{\prime}+\sqrt{\kappa^{\prime 2}+\beta \gamma_{0} \kappa^{\prime \prime}\left(V_{\text {gepw }} V_{g s}\right)^{-0.5}}\right) \kappa^{\prime \prime-1} . \quad$ The overall convective gain for stimulated Raman forward scatter along a parabolic profile is found by inserting the above interaction length into the homogeneous spatial growth rate found above producing 


$$
\Psi_{s}^{*} \alpha \exp \left[\frac{\beta_{1} \gamma_{0}\left(-\kappa^{\prime}+\sqrt{\kappa^{\prime 2}+\beta_{2} \gamma_{o} \kappa^{\prime \prime} / \sqrt{V_{\text {gepw }} V_{g s}}}\right)}{\sqrt{V_{\text {gepw }} V_{g s} \kappa^{\prime \prime}}}\right],
$$

where $\beta_{1}$ and $\beta_{2}$ are constants chosen to match the Rosenbluth results shown in Appendix 2.2. When the perfect phase matching point is far from the top of the parabola, $\kappa^{\prime}>>K^{\prime \prime}$ and the energy gain reduces to $\Psi_{s}^{*} \alpha \exp \left[0.5 \beta_{1} \beta_{2} \gamma_{o}^{2}\left(\kappa^{\top} V_{\text {gepw }} V_{\mathrm{gs}}\right)^{-1}\right]$ which has the correct parameter dependence for a convective instability containing a linear phase mismatch with $\beta_{1} \beta_{2}=4 \pi$ giving the correct energy gain[27]. When the perfect phase mismatch point is at $x=0, K^{\prime}=0$ and the energy gain reduces to $\Psi_{\mathrm{s}}^{*} \alpha \exp \left[\beta_{1} \beta_{2}^{0.5} \gamma_{0}^{1.5}\left(\mathrm{~V}_{\mathrm{gepw}} \mathrm{V}_{\mathrm{gs}}\right)^{-0.75} \kappa^{\prime \prime-0.5}\right]$ which has the correct parameter dependence for a convective instability containing a quadratic mismatch with $\beta_{1} \beta_{2}^{0.5}=6.992$ yielding the correct energy gain[29]. Taking these two limits sets the value of $\beta_{1}$ to 3.89 and $\beta_{2}$ to 3.23. This prescription then allows for the calculation of the gain all along the parabolic profile smoothly going between the top of the parabola, where the phase mismatch is quadratic, and the sides, where the phase mismatch is linear. The wavenumber resonance width including damping can also be used to look at the effects of damping on the gain of convective instabilities which is shown in Appendix 2.3.

Equations 2.1 and 2.2 in Appendix 2.2 were solved numerically to observe the temporal evolution of the waves. The normalization of these equations along with the FORTRAN program written to solve these coupled partial differential equations is shown in Appendix 2.4. Figure 2.3.2 shows the results from four of the simulations performed with this program. In figure 
2.3.2, the initial amplitude and phase of the electron plasma waves were taken to be constant over a distance larger than the interaction length of the instability. The initial amplitude of the slowest wave(solid black line) in all four cases was chosen to be equal to 1.0. In figure 2.3.2 a, the parameter $\gamma_{0}^{2}\left(\kappa^{\top} \mathrm{V}_{\mathrm{gepw}} \mathrm{V}_{\mathrm{gs}}\right)^{-1}$ was set to 1.5 and the ratio of velocities, $\mathrm{V}_{\mathrm{gepw}} \mathrm{V}_{\mathrm{gs}}^{-1}$, was set to $\mathrm{V}_{\text {gepw }} \mathrm{V}_{\mathrm{gs}}^{-1}=1.0$. In figure 2.3.2 $\mathrm{b}$, the parameter $\gamma_{0}^{2}\left(\kappa^{\prime} \mathrm{V}_{\mathrm{gepw}} \mathrm{V}_{\mathrm{gs}}\right)^{-1}$ was set to 1.0 and the ratio of velocities, $\mathrm{V}_{\text {gepw }} \mathrm{V}_{\mathrm{gs}}^{-1}$, was set to $\mathrm{V}_{\mathrm{gepw}} \mathrm{V}_{\mathrm{gs}}^{-1}=1.0$. In figure 2.3.2 c, the parameter $\gamma_{o}^{2}\left(\kappa^{\top} \mathrm{V}_{\mathrm{gepw}} \mathrm{V}_{\mathrm{gs}}\right)^{-1}$ was set to 1.0 and the ratio of velocities, $\mathrm{V}_{\text {gepw }} \mathrm{V}_{\mathrm{gs}}^{-1}$, was set to $\mathrm{V}_{\text {gepw }} \mathrm{V}_{\mathrm{gs}}^{-1}=0.5$. In figure 2.3.2 $\mathrm{d}$, the parameter $\gamma_{o}^{2}\left(\kappa^{\prime} V_{\text {gepw }} V_{\text {gs }}\right)^{-1}$ was set to 1.0 and the ratio of velocities, $V_{\text {gepw }} V_{g s}^{-1}$, was set to $\mathrm{V}_{\mathrm{gepw}} \mathrm{V}_{\mathrm{gs}}^{-1}=0.25$.

The amplitude of the slow wave is simply given by noise level multiplied by the gain factor or $\Psi_{\text {epw }}($ sat $)=\Psi_{\text {epw }}(0) \exp \left(\pi \gamma_{0}^{2}\left(\kappa^{\top} \mathrm{V}_{\text {gepw }} \mathrm{V}_{\mathrm{gs}}\right)^{-1}\right)$. The amplitude of the fast wave is determined by first finding the scattering amplitude produced by the interaction beam scattering from a fixed amplitude slow wave above assuming that the slow wave does not change amplitude. The next step is to multiply this value by the convective gain which in the limit of small gain should reduce to the scattering amplitude found in the first step. The scattering amplitude can be found in a similar manner as the instability gain already covered. In the case of the scattering process, the resonance width is changed. The resonance width for the scattering problem is found by examining the equation

$$
\mathrm{V}_{\mathrm{gs}} \frac{\mathrm{d} \Psi_{\mathrm{s}}^{*}}{\mathrm{dx}}=\gamma_{\mathrm{o}} \Psi_{\mathrm{epw}}(0) \Pi\left(\frac{\mathrm{x}}{2 \mathrm{x}_{\mathrm{int}}}\right)
$$



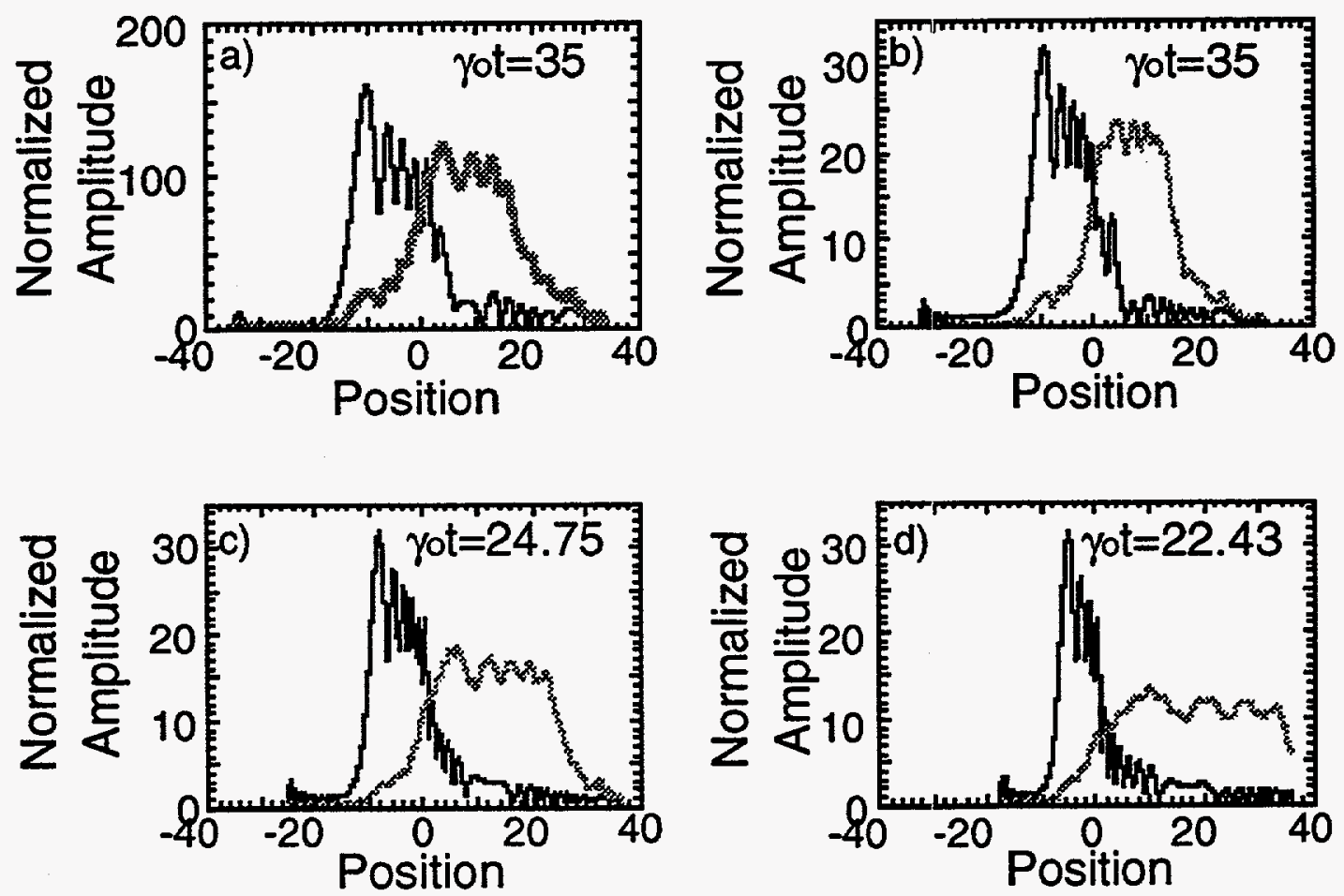

Figure 2.3.2 Numerical solution of the Rosenbluth equations for a linear phase mismatch between the interacting waves. This figure represents four graphs of the numerical solution to the equations 2.2.1 and 2.2.2 shown in Appendix 2.2. In each graph, the vertical axis represents the amplitude that the waves are driven and the horizontal axis represents position. The solid black line in all cases represents a slow wave traveling to the left, while the solid gray line represents a fast wave traveling to the right.

where $\Pi\left(0.5 x_{\text {int }}^{-1}\right)$ is unity between - $x_{\text {int }}$ and $x_{\text {int }}$ and zero elsewhere. $x_{\text {int }}$ represents the length over which the process is driven before saturation. The homogeneous solution to equation 2.3 .5 is simply $\Psi_{\mathrm{s}}^{*}=\Psi_{\mathrm{epw}}(0) \gamma_{\mathrm{o}} \times \mathrm{VV}_{\mathrm{gs}}^{-1}$. The resonance width is found by Fourier transforming equation 2.3.5 and looking 
for the FWHM in the same manner as described above. The Fourier transform of equation 2.3 .5 is

$$
\Psi_{s}^{*}(k)=\frac{i 2 \gamma_{0} \Psi_{\text {epw }}(0) x_{\text {int }}^{2}}{V_{g s}} \frac{\sin \left(k x_{i n t}\right)}{\left(k x_{i n t}\right)^{2}}
$$

It is apparent from equation 2.3.6 that the FWHM is proportional to the inverse of the interaction length or $\Delta \mathrm{k} \propto \mathrm{x}_{\mathrm{int}}^{-1}$. The interaction length is found by equating the resonance width, $\Delta k \propto x_{\text {int }}^{-1}$, with the change in wavenumber, $\Delta \mathrm{k}=\boldsymbol{\kappa}^{\prime} \mathrm{x}_{\mathrm{int}}$, found above. This yields an interaction length proportional to $x_{\text {int }} \propto x^{\prime-1 / 2}$. The scattering amplitude is found by inserting the interaction length into the homogeneous solution above, or

$$
\Psi_{s}^{*}=\sqrt{2 \pi} \Psi_{\text {epw }}(0) \gamma_{0} \kappa^{\prime-0.5} V_{\text {gs' }}^{-1}
$$

where $(2 \pi)^{0.5}$ is the correct numerical coefficient. The amplitude of the fast wave, as determined above, is given by $\Psi_{\mathrm{s}}^{*}=\eta \sqrt{\exp \left[2 \pi \gamma_{o}^{2}\left(\kappa^{\top} \mathrm{V}_{\text {gepw }} \mathrm{V}_{\mathrm{gs}}\right)^{-1}\right]-1}$ where $\eta$ is chosen such that when the term in the exponent is small the amplitude reduces to equation 2.3 .7 above. The amplitude of the fast wave is then given by

$$
\Psi_{\mathrm{S}}^{*}=\mathrm{V}_{\mathrm{gepw}}^{0.5} \mathrm{~V}_{\mathrm{gs}}^{-0.5} \sqrt{\exp \left[2 \pi \gamma_{0}^{2}\left(\kappa^{\prime} \mathrm{V}_{\mathrm{gepw}} \mathrm{V}_{\mathrm{gs}}\right)^{-1}\right]-1}
$$


Equation 2.3.8 agrees very well with the numerical results shown in figure 2.3.2 above.

There have been many experiments to verify the convective growth of parametric instabilities[30,31]. One of these experiments[30] involved looking at the growth of forward Raman scatter at low enough densities such that backward Raman scattering could not occur, thereby preventing coupling between these instabilities which could lead to absolute instability[32,33]. The results of this experiment are shown in figure 2.3.3 below. This figure shows the gain has the exponential dependence on the pump intensity expected

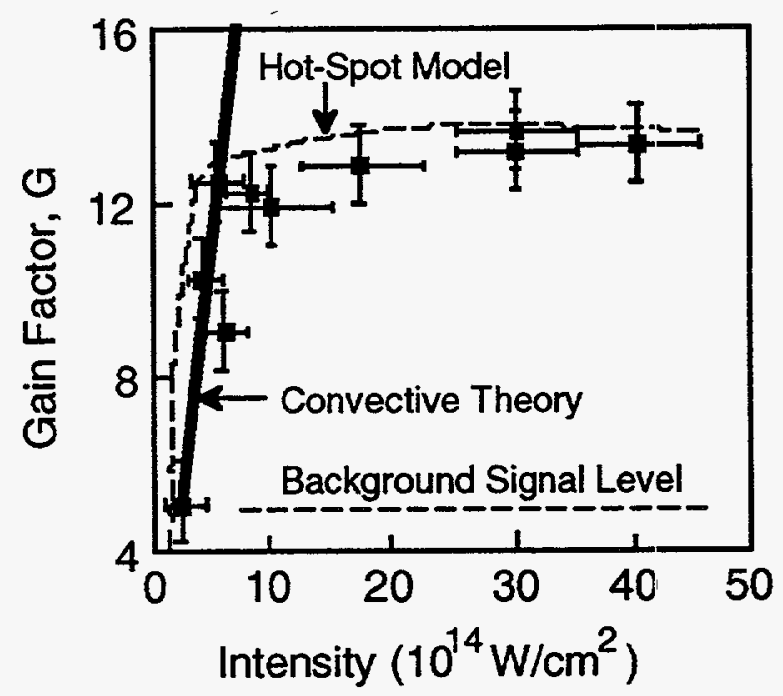

Figure 2.3.3 Measured amplification of the scattered electromagnetic wave associated with stimulated Raman forward scattering as a function of pump intensity. This graph has been reprinted from Batha et al.[34]. 
from convective saturation only for intensities less than approximately $7 \times 10^{14}$ $\mathrm{W} / \mathrm{cm}^{2}$. Above pump intensities of $7 \times 10^{14} \mathrm{~W} / \mathrm{cm}^{2}$, the Raman process is being saturated by some other mechanism.

The overall convective gain experienced by the daughter waves participating in stimulated Raman backscattering would predict an increase in gain with a decrease in the parameter $\mathrm{kepw}_{\mathrm{e}} \lambda_{\mathrm{De}}$ which is in contrast to experimental observations such as the one shown in figure 2.10.3. The only way to salvage the convective saturation is to assume that the noise level from which these instabilities grow decreases sufficiently with $\mathrm{k}_{\mathrm{epw}} \lambda_{\mathrm{De}}$ to explain the experimental spectrum which was not found to be the case[35]. This suggests that stimulated Raman backscattering is not being saturated due to the detuning as the waves travel along the plasma inhomogeneity.

\subsection{Pump depletion}

Pump depletion can occur when the daughter waves associated with a parametric instability grow to a sufficient amplitude that a significant fraction of the energy contained in the pump wave is transferred to the daughter waves. In this way, the amplitude of the daughter waves can saturate because of the corresponding reduction in the growth rate which is proportional to the pump intensity of the interaction beam. To account for pump depletion, the equation describing the pump beam must be included along with the equations describing each of the daughter waves. The pump beam equation then contains a term which provides a sizable damping to the pump beam 
when the daughter waves become large enough to remove a significant fraction of the pump beam's energy.

In many small scale experiments, the level of stimulated Raman scattering driven in the plasma is quite small, typically representing less than $10^{-3}$ to $10^{-5}$ of the incident lasers energy, however, the energy contained in stimulated Brillouin scattering can be on the order of $10^{-1}$. The effect of pump depletion in an inhomogeneous plasma has been looked at previously[36]. The following calculation will look at the effectiveness of stimulated Brillouin scattering in depleting the pump in a homogeneous plasma. The equations describing stimulated Brillouin scattering can be reduced to first order partial differential equations by making the slowly varying envelope approximation as shown in appendix 2.2. In addition by looking for a steadystate solution, the three equations describing stimulated Brillouin scattering are then reduced to three ordinary differential equations plus their complex conjugates. These equations can be combined to yield the Manley-Rowe relations

$$
V_{g s} \frac{d\left|\Psi_{s}(x)\right|^{2}}{d x}=-V_{g o} \frac{\omega_{o}}{\omega_{s}} \frac{d\left|\Psi_{o}(x)\right|^{2}}{d x}=V_{g i} \frac{\omega_{i}}{\omega_{s}} \frac{M_{i}}{Z m_{e}} \frac{\omega_{p e}^{2}}{k_{i}^{2} c^{2}} \frac{d\left|\Psi_{i}(x)\right|^{2}}{d x}
$$

The Manley-Rowe relations can then be integrated to provide the relationship between the pump wave, $\Psi_{\mathrm{o}}(\mathrm{x})$, and the ion wave, $\Psi_{\mathrm{i}}(\mathrm{x})$, in terms of the scattered wave, $\Psi_{s}(\mathrm{x})$. The pump wave can expressed as $\left|\Psi_{\mathrm{o}}(\mathrm{x})\right|^{2}=\phi_{o}^{2}-\frac{\omega_{\mathrm{s}} \mathrm{V}_{\mathrm{gs}}}{\omega_{\mathrm{o}} \mathrm{V}_{\mathrm{go}}}\left|\Psi_{\mathrm{s}}(\mathrm{x})\right|^{2}$, and the ion wave can be expressed as 
$\left|\Psi_{\mathrm{i}}(\mathrm{x})\right|^{2}=\frac{\omega_{\mathrm{s}} \mathrm{V}_{\mathrm{gs}} \mathrm{Zm} \mathrm{m}_{\mathrm{e}} \mathrm{k}_{\mathrm{i}}^{2} \mathrm{c}^{2}}{\omega_{\mathrm{i}} \mathrm{V}_{\mathrm{gi}} \mathrm{M}_{\mathrm{i}} \omega_{\mathrm{pe}}^{2}}\left|\Psi_{\mathrm{s}}(\mathrm{x})\right|^{2}$. The initial six ordinary differential equations can be combined with the above two relations to form a second order differential equation representing the growth of the scattered light wave

$$
\left.\frac{\mathrm{d}^{2}\left|\Psi_{s}(\mathrm{x})\right|^{2}}{\mathrm{dx} \mathrm{x}^{2}}=-\left.\left(\frac{6 \gamma_{0}^{2} \omega_{\mathrm{s}} \mathrm{V}_{\mathrm{gs}}}{\phi_{0}^{2} \mathrm{~V}_{\mathrm{gs}} \mathrm{V}_{\mathrm{gi}} \omega_{\mathrm{o}} \mathrm{V}_{\mathrm{go}}}\right)|| \Psi_{\mathrm{s}}(\mathrm{x})\right|^{2}\right]^{2}+\left|\Psi_{\mathrm{s}}(\mathrm{x})\right|^{2}\left(\frac{4 \gamma_{0}^{2}}{\mathrm{~V}_{\mathrm{gs}} \mathrm{V}_{\mathrm{gi}}}\right)
$$

Provided $\left|\Psi_{s}(x)\right|^{2} \ll \phi_{0}^{2}$, equation 2.4 .2 shows the correct gain for the scattered light of $\left|\Psi_{s}(x)\right|^{2} \propto \exp \left[2 \gamma_{0} x\left(V_{g i} V_{g s}\right)^{-0.5}\right]$. Saturation occurs when the scattered light wave has reached the value $\left|\Psi_{s}(x)\right|^{2}=\left(2 \omega_{0} V_{g o} / 3 \omega_{s} V_{g s}\right) \phi_{o}^{2}$. For backscattered stimulated Brillouin scattering, pump depletion saturates the parametric process when the scattered light wave has reached 67 percent of the incident wave.

As stated above, many small-scale experiments measure reflectivities of stimulated Brillouin scattering on the order of 10 percent. These absolute measurements are spatially and temporally integrated over the entire plasma. Therefore, the spatial and temporal region over which stimulated Brillouin scattering is being driven could be saturated by pump depletion and would therefore affect the other parametric instabilities as well. Also recent shortpulse Raman experiments have measured tens of percents of Raman reflectivities which might be in the range where pump depletion is operating[37]. In many short-pulse high-intensity experiments the laser is less than a picosecond in duration and consequently the daughter waves can grow to saturation levels faster than many of the saturation mechanisms 
involving ion waves described below can occur. In these cases pump depletion and particle trapping, discussed below in section 2.10, can become important saturation mechanisms.

\subsection{Secondary decay processes}

The experiments performed for this dissertation primarily cover saturation of parametric instabilities due to secondary decay processes. Secondary decay processes occur when the decay waves associated with a parametric instability become large enough that they can in turn become the pump wave for a secondary decay process. As the daughter waves driven by the secondary instability grow, the primary instability may saturate if enough energy is removed to pump the secondary instability. The secondary instability, therefore, introduces an effective "nonlinear" damping into the primary instability[9]. In addition, parametric instabilities may saturate due to the frequency shift resulting from the coupling to secondary instabilities and also due to the self consistent detuning caused by changes to the background density $[17,38]$.

The most widely studied of the secondary decay processes is the Langmuir decay instability $[9,12,13,39]$. The Langmuir decay instability involves the decay of a "pump" Langmuir wave into a second Langmuir wave and an ion acoustic wave. The experiments performed for this dissertation have implications primarily for this saturation mechanism. In the case of stimulated Raman scattering, the incident electromagnetic wave drives an electromagnetic wave and a Langmuir wave. When the Langmuir 
wave becomes large enough, it can overcome the damping threshold and drive a second Langmuir wave and an ion acoustic wave, the Langmuir decay instability. The experimental evidence for this saturation mechanism is shown primarily in Chapter 6 , but also in chapter 5 and in Villeneuve et al.,[40].

Although the Langmuir decay instability has received the most attention, there are a number of other possible decays. The Langmuir wave associated with stimulated Raman scattering can also decay into an electromagnetic wave and an ion acoustic wave, the electromagnetic decay instability,[41-45] which is discussed in detail in chapter 3. The threshold conditions for the Langmuir decay instability and the electromagnetic decay instability can be expressed as conditions on the amplitude, $\delta n / n$, of the pump Langmuir wave

$$
\begin{aligned}
& \left.\frac{\delta \mathrm{n}}{\mathrm{n}}\right|_{\mathrm{LDI}} \approx \frac{4 \mathrm{k}_{\mathrm{epw}} \lambda_{\mathrm{De}}}{\left(\hat{\mathrm{e}}_{\mathrm{epw}} \cdot \hat{\mathrm{e}}_{\mathrm{epw} 2}\right)} \sqrt{\frac{v_{\mathrm{ia}}}{\omega_{\mathrm{ia}}} \frac{v_{\mathrm{epw}}}{\omega_{\mathrm{epw}}}} \text { and } \\
& \left.\frac{\delta \mathrm{n}}{\mathrm{n}}\right|_{\mathrm{EDI}} \approx \frac{4 \mathrm{k}_{\mathrm{epw}} \lambda_{\mathrm{De}}}{\left(\hat{\mathrm{e}}_{\mathrm{epw}} \cdot \hat{\mathrm{e}}_{\mathrm{s}}\right)} \sqrt{\frac{v_{\mathrm{ia}}}{\omega_{\mathrm{ia}}} \frac{v_{\mathrm{s}}}{\omega_{\mathrm{s}}}}
\end{aligned}
$$

respectively[39,46]. In equation 2.5 .1 and 2.5 .2 above, $k_{e p w}$ is the wavenumber of the pump Langmuir wave, $\omega_{\mathrm{ia}}\left(v_{\mathrm{ia}}\right) \omega_{\mathrm{epw}}\left(v_{\mathrm{epw}}\right)$, and $\omega_{\mathrm{S}}\left(v_{\mathrm{S}}\right)$ are the frequencies(damping) of the daughter ion wave, Langmuir wave, and electromagnetic wave, respectively, and $\hat{e}_{\text {epw }}$ and $\hat{e}_{\text {epw2 }}$, are unit vectors in the direction of propagation for the pump Langmuir wave, $\hat{\mathrm{e}}_{\mathrm{epw}}$, and the daughter Langmuir, $\hat{e}_{\text {epw2 }}$, wave while $\hat{\mathrm{e}}_{\mathrm{s}}$ is the unit vector in the direction 
of the vector potential of the scattered electromagnetic wave. Figure 2.5.1 shows the threshold amplitude which the Raman generated Langmuir wave must reach before the threshold for convective instability is reached. This figure assumes a $\mathrm{CH}$ plasma with an electron temperature of $1 \mathrm{keV}$ and an incident laser wavelength of $351 \mathrm{~nm}$. The solid line represents the threshold corresponding to the Langmuir decay instability while the dashed line represents the threshold corresponding to the electromagnetic decay instability. This figure shows that the Langmuir decay instability has a slightly lower Langmuir-wave-amplitude threshold for $k \lambda_{D e}$ less than approximately. 0.2 , however, the electromagnetic decay instability has a substantially lower Langmuir-wave-amplitude threshold for $k \lambda_{D e}>0.2$. The daughter electromagnetic wave associated with the electromagnetic decay instability does not experience Landau damping, so when the Landau damping on the daughter Langmuir wave associated with the Langmuir decay instability becomes larger than the collisional damping, the threshold for the Langmuir decay instability rises exponentially due to the Landau damping on the daughter Langmuir wave. This results in a higher threshold for the Langmuir decay instability than the electromagnetic decay instability for $k \lambda_{\mathrm{De}}>0.2$. The threshold for the Langmuir decay instability qualitatively agrees with representative spectrum of stimulated Raman backscattering which can be seen in figure 2.10 .3 below. The threshold for LDI is highest for $k \lambda_{\mathrm{De}}>0.2$ when Landau damping on the daughter Langmuir wave is large, however, it falls quickly as $k \lambda_{\text {De }}$ decreases due to the exponential dependence on $k \lambda_{\text {De }}$ for the threshold condition which is similar to the spectrum shown 
in figure 2.10.3. This qualitative behavior was the basis for an explanation of the gap region in the Raman spectrum discussed in section $2.8 \mathrm{~b}$ [39].

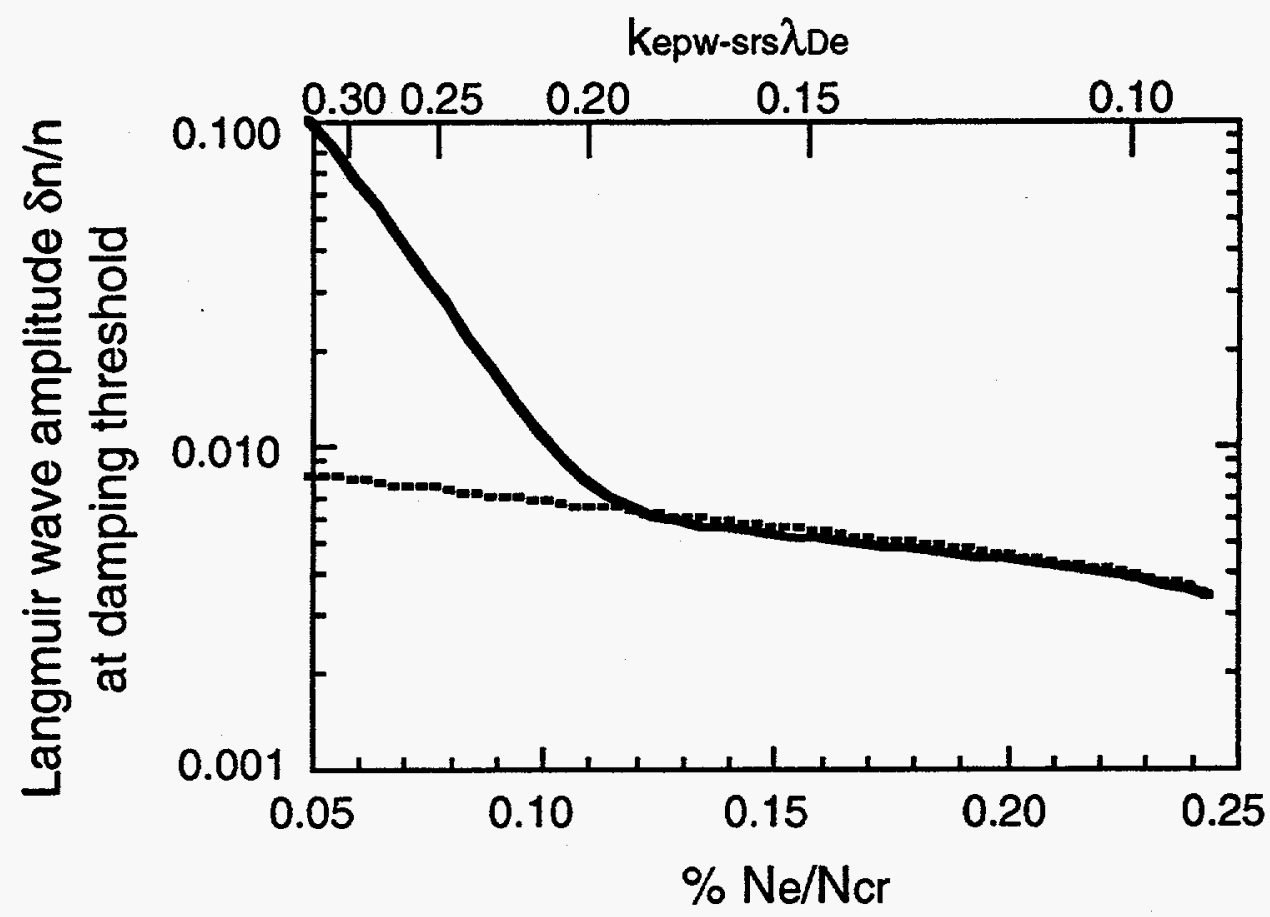

Figure 2.5.1 Figure showing the amplitude of the Langmuir wave at the threshold for convective instability. The solid line represents the threshold for the Langmuir decay instability while the dashed line represents the threshold for instability of the electromagnetic decay instability. The bottom horizontal axis shows the percent of critical density that the Raman process is occurring, while the top horizontal axis shows the corresponding value of $k \lambda_{\text {De }}$ for the direct backscattered Raman generated Langmuir wave.

The driven electromagnetic wave in stimulated Raman scattering can also undergo secondary decay. The range of densities over which Raman scattering is driven produces scattered electromagnetic waves which range 
from the scattered electromagnetic waves critical surface down to well below the quarter-critical-surface for the scattered electromagnetic wave. The secondary decay processes which may be driven by the scattered electromagnetic wave associated with stimulated Raman scattering therefore includes the oscillating two-stream instability at the quarter critical surface, the ion acoustic decay instability from $n_{e} / n_{c r}=0.25$ to 0.2 , two plasmon decay from $n_{e} / n_{c r}=0.09$ to 0.1 , stimulated Brillouin scattering below $n_{e} / n_{c r}=0.25$, and stimulated Raman scattering below $n_{e} / n_{c r}=0.1$ where these densities are in relation to the electromagnetic wave driving the stimulated Raman scattering process.

The easiest of these secondary processes, resulting from the scattered electromagnetic wave, to observe would be the decay of the scattered electromagnetic wave associated with stimulated Raman scattering into two Langmuir waves, two plasmon. decay. This should occur at approximately $n_{e} / n_{c r}=0.09$ to 0.1 with respect to the frequency of the interaction beam driving the stimulated scattering process. The secondary Langmuir waves would then be observed as the interaction beam Thomson scatters from the Langmuir waves driven by this process producing a scattered electromagnetic wave which should be easily diagnosed. The scattered electromagnetic spectrum should have the characteristic double peaked emission pattern associated with $3 / 2 \omega_{0}$ emission. In this case, however, the double peak pattern would be centered about $1.32 \omega_{\mathrm{o}}$. This experiment was proposed as a UC-LLNL collaborative experiment on the NOVA laser. This process could be important for holhraum experiments which have very long scalelengths and are typically filled with a gas mixture at about ten percent of the critical 
density for the interaction beam, the required density for this process to occur. Large levels of stimulated Raman scattering, $>10 \%$, have been observed in such experiments indicating that this particular secondary instability should be above the required threshold. If this experiment were done in a gas bag or holhraum configuration, the damping on the Langmuir waves could be easily varied by changing the ionic concentration of the gas present.

\subsection{Modulational instability and collapse of the Langmuir waves}

The modulational instability is another mechanism which could be considered a secondary decay mechanism mentioned above. In the modulational instability, a Langmuir wave decays into a purely growing density fluctuation plus a number of secondary Langmuir waves[47-49]. The equations, which represent a sufficient description of the modulational instability, are known as the Zakharov equations and are derived in Appendix 2.5 for the case of ponderomotive detuning. The low frequency Zakharov equation[50] is given by

$$
\left(\frac{\partial^{2}}{\partial t^{2}}-c_{s}^{2} \nabla^{2}\right) \frac{n_{e l}}{n_{o e}}=\frac{Z m_{e}}{2 M_{i}} \nabla^{2}\left|u_{e h}\right|^{2} .
$$

The high frequency Zakharov equation can be written as

$$
\left(\mathrm{i} \frac{\partial}{\partial \mathrm{t}}+\frac{3}{2} \omega_{\mathrm{pe}} \lambda_{\mathrm{pe}}^{2} \nabla^{2}\right) \mathrm{u}_{\mathrm{eh}}=0.5 \omega_{\mathrm{pe}} \frac{\mathrm{n}_{\mathrm{el}}}{\mathrm{n}_{\mathrm{oe}}} \mathrm{u}_{\mathrm{eh}}
$$


where the fast oscillation at the plasma frequency, $\omega_{\mathrm{pe}}$, has been removed from the equation. These two equations can be Fourier transformed yielding the dispersion relation for the modulational instability. Including both a stokes and anti-stokes daughter Langmuir wave, the dispersion relation for the modulational instability can be written as

$$
\begin{aligned}
{\left[\frac{\omega^{2}}{\omega_{\mathrm{pe}}^{2}}-\frac{\mathrm{c}_{\mathrm{s}}^{2}}{\mathrm{v}_{\mathrm{th}}^{2}} \mathrm{k}^{2} \lambda_{\mathrm{pe}}^{2}\right]=} & \frac{\mathrm{Zm} \mathrm{e}}{\mathrm{M}_{\mathrm{i}}} \frac{\mathrm{k}^{2} \mathrm{v}_{\mathrm{os}}^{2} \cos ^{2} \theta}{8 \omega_{\mathrm{pe}}^{2}} \\
& {\left[\left\{-\frac{\omega \omega_{0}}{\omega_{\mathrm{pe}}^{2}}+\frac{\omega^{2}}{2 \omega_{\mathrm{pe}}^{2}}-1.5 \lambda_{\mathrm{De}}^{2}\left(-2 \overrightarrow{\mathrm{k}}_{\mathrm{o}} \cdot \overrightarrow{\mathrm{k}}+\mathrm{k}^{2}\right)\right\}^{-1}+\right.} \\
& \left.\left\{\frac{\omega \omega_{0}}{\omega_{\mathrm{pe}}^{2}}+\frac{\omega^{2}}{2 \omega_{\mathrm{pe}}^{2}}-1.5 \lambda_{\mathrm{De}}^{2}\left(2 \overrightarrow{\mathrm{k}}_{\mathrm{o}} \cdot \overrightarrow{\mathrm{k}}+\mathrm{k}^{2}\right)\right\}^{-1}\right]
\end{aligned}
$$

This dispersion relation also describes the Langmuir decay instability, in which case the antistokes component is non-resonant and can be neglected. In the dipole limit of the pump Langmuir wave, $k_{0}=0$, a range of ion wavenumbers are unstable, causing a broadening in the Langmuir wave spectrum. For the experiments described in chapters 5 and 6 which saw a broadening in the Langmuir wave spectrum, the dipole approximation for the pump Langmuir wave is, however, not a good assumption since in these experiments $k_{0} \lambda_{D e} \approx 0.15$ to 0.3 . In this case, the finite pump wavenumber has a stabilizing effect on the modulational instability reducing the range of unstable wavenumbers as shown below in figure 2.6.1. The modulational instability might also play an important role in increasing the level which 
stimulated Raman scattering can grow. In section 2.3, it was the effects of inhomogeneity on the overall gain was reviewed. A simple model was constructed which showed that the gain was limited by the finite wavenumber resonance width driven by the instability. This finite resonance width limited the length over which stimulated Raman scattering could grow due to the detuning effects of the plasma inhomogeneity. The modulational instability, however, increases the resonance width which could lead to a larger interaction distance and a correspondingly larger gain.

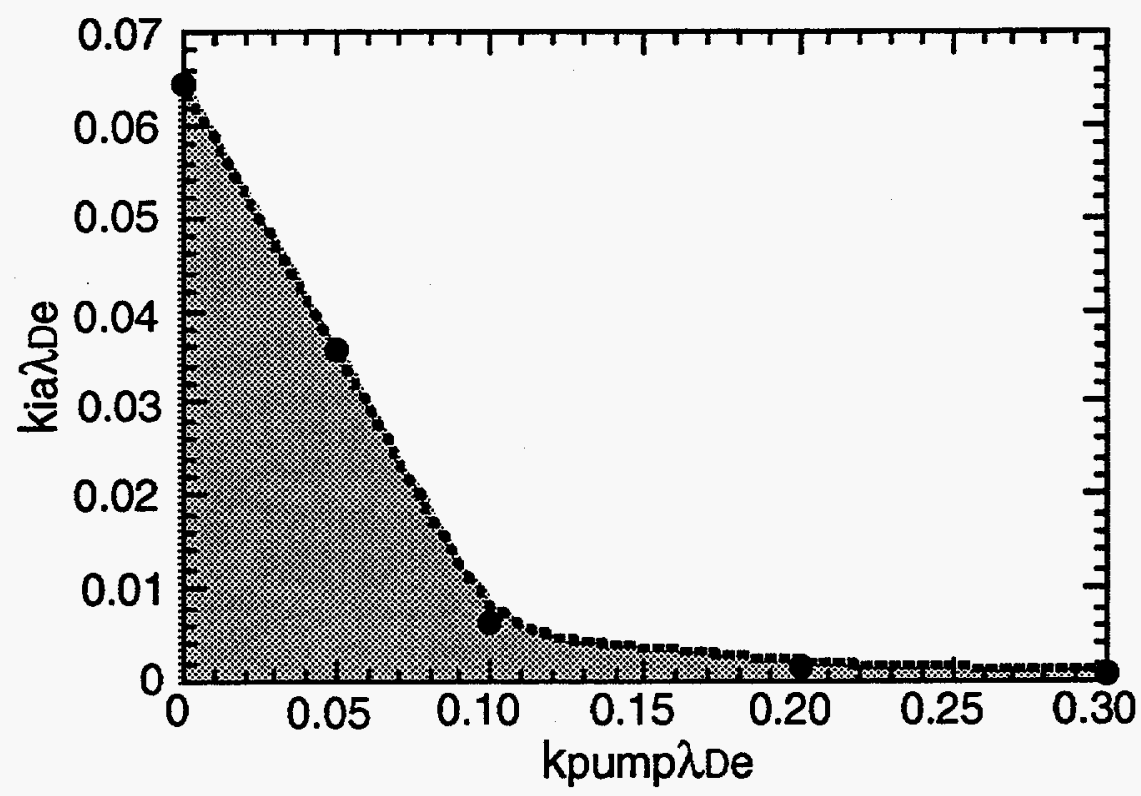

Figure 2.6.1 Range of unstable ion wavenumbers, $k_{i a}$, driven by the modulational instability. The horizontal axis represents the wavenumber of the pump Langmuir wave while the vertical axis represents the wavenumber of the ion wave. The shaded region shows the unstable ion wavenumbers. This graph was made assuming a pump Langmuir amplitude of $\delta n / n=0.01$. 
There have been several experiments which have looked at the effect of modulational instability in laser-produced plasmas[3,51]. The two experiments referenced above used Thomson scattering to observe the Langmuir wave and ion wave spectrum driven by two copropagating electromagnetic waves whose frequency difference was approximately equal to the plasma frequency. These experiments reported that the modulational instability was the mechanism responsible for the saturation of the amplitude of the Langmuir wave driven by these two copropagating electromagnetic waves.

When the amplitude of the Langmuir waves become large enough, the Langmuir waves can become trapped in the density hole, caviton, dug by the ponderomotive force associated with the Langmuir wave itself. The threshold amplitude for caviton formation can be understood by a simple pressure balance argument. The pressure associated with the electric field of the Langmuir wave pushes plasmas out of regions of high field in order to maintain a balance of pressure with the surrounding plasma. The threshold for caviton formation is the point at which the frequency of the Langmuir wave inside the caviton becomes equal to the electron plasma frequency outside of the caviton, at which point the Langmuir wave becomes trapped. Assuming that the frequency of the Langmuir wave is equal to the plasma frequency outside of the caviton, the ratio of the density inside the cavity to that outside the cavity is simply $n_{\text {inside }} / n_{\text {outside }}=\omega_{\mathrm{pe}}^{2} / \omega_{\text {epw }}^{2}$. Using the dispersion relation for a Langmuir wave, $\omega_{\mathrm{epw}}^{2}=\omega_{\mathrm{pe}}^{2}\left(1+3 \mathrm{k}_{\mathrm{epw}}^{2} \lambda_{\mathrm{De}}^{2}\right)$, this ratio may be written as $n_{\text {inside }} / n_{\text {outside }}=1-3 k_{\text {epw }}^{2} \lambda_{\text {De }}^{2}$. The pressure balance then 
equates the field, $E^{2} / 8 \pi$, and particle pressure, $n_{\text {inside }} K T_{\text {inside, }}$ inside the caviton with the particle pressure, $\mathrm{n}_{\text {outside }} \mathrm{kT}$ outside, outside the caviton,

$$
\mathrm{n}_{\text {inside }} K T_{\text {inside }}+E^{2} / 8 \pi=n_{\text {outside }} K T_{\text {outside }}
$$

Using the above relations and assuming that the electron temperature inside the caviton is equal to the temperature outside the caviton, the pressure balance can be written as $E^{2} /\left(8 \pi n_{\text {outside }} \kappa T\right)=3 k_{\text {epw }}^{2} \lambda_{\text {De }}^{2}$. Utilizing Gauss' law, $\nabla \cdot \mathrm{E}=4 \pi \rho$, the above pressure balance can be written in terms of the Langmuir wave amplitude, $\mathrm{n}_{\text {epw }} / \mathrm{n}_{\text {inside }}=\sqrt{6} \mathrm{k}_{\text {epw }}^{2} \lambda_{\text {De }}^{2}$.

Zakharov showed that in 3-dimensions equations 2.6.1 and 2.6.2 lead to the collapse of the Langmuir waves, resulting in a further localization of the Langmuir wave energy[50,52]. The self-accelerating collapse process continues until the caviton has reached the size where a near thermal electron can traverse the caviton in a half cycle of the Langmuir waves frequency. At this point the energy contained in the Langmuir wave is quickly returned to the electrons through transit time damping and the caviton "burns out"[47]. The saturation of the Langmuir wave amplitude in this case is determined by the amplitude of the Langmuir wave directly before the "burn out" stage of the collapse. The saturation of the Raman process, however, could occur before the saturation of the Langmuir wave itself. Although the amplitude of the Langmuir wave is growing, it is becoming localized over an ever decreasing volume. The energy density of the scattered light is proportional to the square of the amplitude of the density fluctuation multiplied by the square of the typical dimension of the caviton. In the self-similar solution to the Zakharov 
equations in 3-d, the amplitude of the Langmuir wave is proportional to the inverse square of the size of the caviton. This solution, however, is for the case of an undriven Langmuir wave. In that case, the total energy of scattered light from the caviton is proportional to the caviton size during collapse. The maximum scattered light detected in the experiment would then be determined from the amplitude of the Langmuir wave at the onset of collapse which was given above to be approximately $\mathrm{n}_{\text {epw }} / \mathrm{n}_{\text {inside }}=\sqrt{6} \mathrm{k}_{\mathrm{epw}}^{2} \lambda_{\mathrm{De}}^{2}$. This would predict a smaller amplitude Langmuir wave at saturation than the threshold for Langmuir decay instability discussed above only for $k_{e p w} \lambda_{D e}<0.03$. Again, this does not correspond to the saturation of the amplitude of the Langmuir wave which continues to grow over an ever decreasing volume until the "burn out" phase of the caviton. This also does not include the growth of the Langmuir wave due to the Raman process itself which could cause the increase scattering due to the higher Langmuir wave amplitude be more important than the reduction in scattering volume.

During the collapse of the caviton, the frequency of the Langmuir waves is also changing which can remove the Langmuir waves out of the resonance width driven by the stimulated Raman process. The detuning resulting from the amplitude dependent change in the frequency of the Langmuir wave is discussed in the following section. Once the Langmuir wave has been removed from the resonance, the growth of the Langmuir wave is determined by the dynamics of the collapsing caviton and the above estimations should be valid. 


\subsection{Ponderomotive detuning}

Ponderomotive detuning was proposed as a saturation mechanism for stimulated Raman forward scattering[38]. This mechanism uses the Zakharov equations described above and looks at the effect of the change in frequency of the Langmuir wave, caused by the growth of the Langmuir wave, on the growth of stimulated Raman forward scatter. In the paper introducing this mechanism, [38] the Zakharov equations were solved in steady-state using the quasi-static approximation with the further assumption that the spatial dependence could be written as a slowly varying component multiplied by a fast oscillation in the same manner as the convective instabilities in section 2.3. Solving these equations in steady-state precludes the possibility of Langmuir collapse. The modulational instability, which causes a broadening in the wavenumber spectrum of the Langmuir waves, might stabilize this saturation mechanism in the sense that although the spectrum is changing, if the overall spectrum is broad enough that there is an overlap with the original resonance width, the instability might continue to grow.

The effect of ponderomotive detuning can be viewed very simply by examining the equation describing the Langmuir wave participating in stimulated Raman scattering with the inclusion of the Zakharov low frequency response, 


$$
\begin{aligned}
& {\left[\frac{\partial^{2}}{\partial t^{2}}+\omega_{\mathrm{pe}}^{2}\left\{1-\frac{1}{2}\left(\frac{1}{k_{\mathrm{epw}}^{2} \lambda_{\mathrm{oe}}^{2}}\left|\frac{\mathrm{n}_{\mathrm{epw}}}{\mathrm{n}_{\mathrm{oe}}}\right|^{2}+\frac{\mathrm{c}^{2}}{\mathrm{v}_{\mathrm{th}}^{2}}\left|\frac{\mathrm{qA} \mathrm{A}_{\mathrm{scatt}}}{\mathrm{m}_{\mathrm{e}} \mathrm{c}^{2}}\right|^{2}\right)\right\}-\frac{3 \kappa \mathrm{T}_{\mathrm{e}}}{\mathrm{m}_{\mathrm{e}}} \nabla^{2}\right]} \\
& \frac{\mathrm{n}_{\mathrm{epw}}}{\mathrm{n}_{\mathrm{oe}}}=c^{2} \nabla^{2}\left(\frac{\mathrm{qA} \mathrm{A}_{\mathrm{scatt}}}{\mathrm{m}_{\mathrm{e}} \mathrm{c}^{2}} \cdot \frac{\mathrm{qA} \mathrm{A}_{\mathrm{pump}}}{\mathrm{m}_{\mathrm{e}} \mathrm{c}^{2}}\right),
\end{aligned}
$$

which is identical to the expression derived in Appendix 2.1 except for the nonlinear term on the left side of the equation which accounts for the effect of the Langmuir wave and scattered electromagnetic wave pressure on the plasma frequency. This process can be thought of as a detuning by removing the frequency of the wave from the resonance width driven by the instability. The instability is driven only over a narrow range of frequencies proportional to the homogeneous growth rate of the instability. Ignoring the electromagnetic pressure, the left hand side of the above equation can be expressed roughly as the dispersion relation

$$
\omega_{\text {epw }}^{2}+\omega_{\mathrm{pe}}^{2}\left(1-\frac{1}{2 k_{\mathrm{epw}}^{2} \lambda_{\mathrm{pe}}^{2}}\left|\frac{\mathrm{n}_{\mathrm{epw}}}{\mathrm{n}_{\mathrm{oe}}}\right|^{2}\right)+3 \mathrm{v}_{\mathrm{th}}^{2} \mathrm{k}_{\mathrm{epw}}^{2} \approx 0
$$

with the assumption that when $\mathrm{nepw}_{\mathrm{e}}\left(\mathrm{n}_{\mathrm{oe}}\right)^{-1}$ is small, $\omega_{\mathrm{epw}}^{2}+\omega_{\mathrm{pe}}^{2}+3 \mathrm{v}_{\mathrm{th}}^{2} \mathrm{k}_{\mathrm{epw}}^{2} \approx 0$ is satisfied. As the Langmuir wave grows in amplitude, the wavenumber of the Langmuir wave frequency changes, $\mathrm{k}_{\text {epw }} \Rightarrow \mathrm{k}_{\text {epw }}+\Delta \mathrm{k}$. By expanding the dispersion relation, this change in wavenumber is found to be proportional to $\Delta \mathrm{k} \propto\left|\mathrm{n}_{\mathrm{epw}}\left(\mathrm{n}_{\mathrm{oe}}\right)^{-1}\right|^{2} \omega_{\mathrm{pe}}\left[\left(\mathrm{k}_{\mathrm{epw}}^{2} \lambda_{\mathrm{De}}^{2}\right) \mathrm{V}_{\mathrm{epw}}\right]^{-1}$. Using the results from section 2.3, the wavenumber resonance width is proportional to $\Delta \mathrm{k} \propto \gamma_{\mathrm{o}}\left(\mathrm{V}_{\mathrm{epw}} \mathrm{V}_{\mathrm{s}}\right)^{-0.5}$. 
Once the wavenumber changes by a number of resonance widths, the instability is detuned and ceases to grow. Therefore, the amplitude of the Langmuir wave, $n_{\text {epw }}\left(n_{\text {oe }}\right)^{-1}$, saturates at $\left|\mathrm{n}_{\mathrm{epw}}\left(\mathrm{n}_{\mathrm{oe}}\right)^{-1}\right| \propto \mathrm{k}_{\mathrm{epw}} \lambda_{\mathrm{De}} \gamma_{\mathrm{oSRS}}^{0.5} \omega_{\mathrm{pe}}^{-0.5} \mathrm{~V}_{\mathrm{epw}}^{0.25} \mathrm{~V}_{\mathrm{s}}^{-0.25}$. Appendix 2.5 details the calculation and finds the numerical coefficient in front which is equal to 0.5 . Figure 2.7.1 below shows a comparison of the Langmuir wave amplitude predicted by the ponderomotive detuning model with the Langmuir wave amplitude at the damping threshold for the Langmuir decay instability. This figure was generated assuming a $\mathrm{CH}$ plasma at $1 \mathrm{keV}$ irradiated with a $351 \mathrm{~nm}$ interaction beam at $5 \times 10^{14} \mathrm{~W} / \mathrm{cm}^{2}$. As shown in the figure, the ponderomotive detuning can onset at much smaller values than the damping threshold for the Langmuir decay instability. However, as stated above this treatment assumes that the modulational instability does not broaden the Langmuir wave spectra which might prevent saturation of the Langmuir wave's amplitude by this mechanism. In addition, many of the simulations looking at the saturation of stimulated Raman scattering, which include the physics of the Langmuir decay instability and ponderomotive detuning, see the generation of Langmuir waves attributed to the Langmuir decay instability $[9,12,13]$.

This mechanism can also be applied to the problem of beat wave excitation of Langmuir waves[2]. In this case, the nonlinear dispersion relation remains the same, however, the wavenumber resonance width of the scattering process is different than the stimulated process above. Again, by expanding the nonlinear dispersion relation shown in 2.7.2, the change in wavenumber resulting from the ponderomotive force of the Langmuir wave 
is proportional to $\Delta \mathrm{k} \propto\left|\mathrm{n}_{\mathrm{epw}}\left(\mathrm{n}_{\mathrm{oe}}\right)^{-1}\right|^{2} \omega_{\mathrm{pe}}\left[\left(\mathrm{k}_{\mathrm{epw}}^{2} \lambda_{\mathrm{pe}}^{2}\right) \mathrm{V}_{\mathrm{epw}}\right]^{-1}$, the same as the stimulated process described above. The resonance width for the scattering

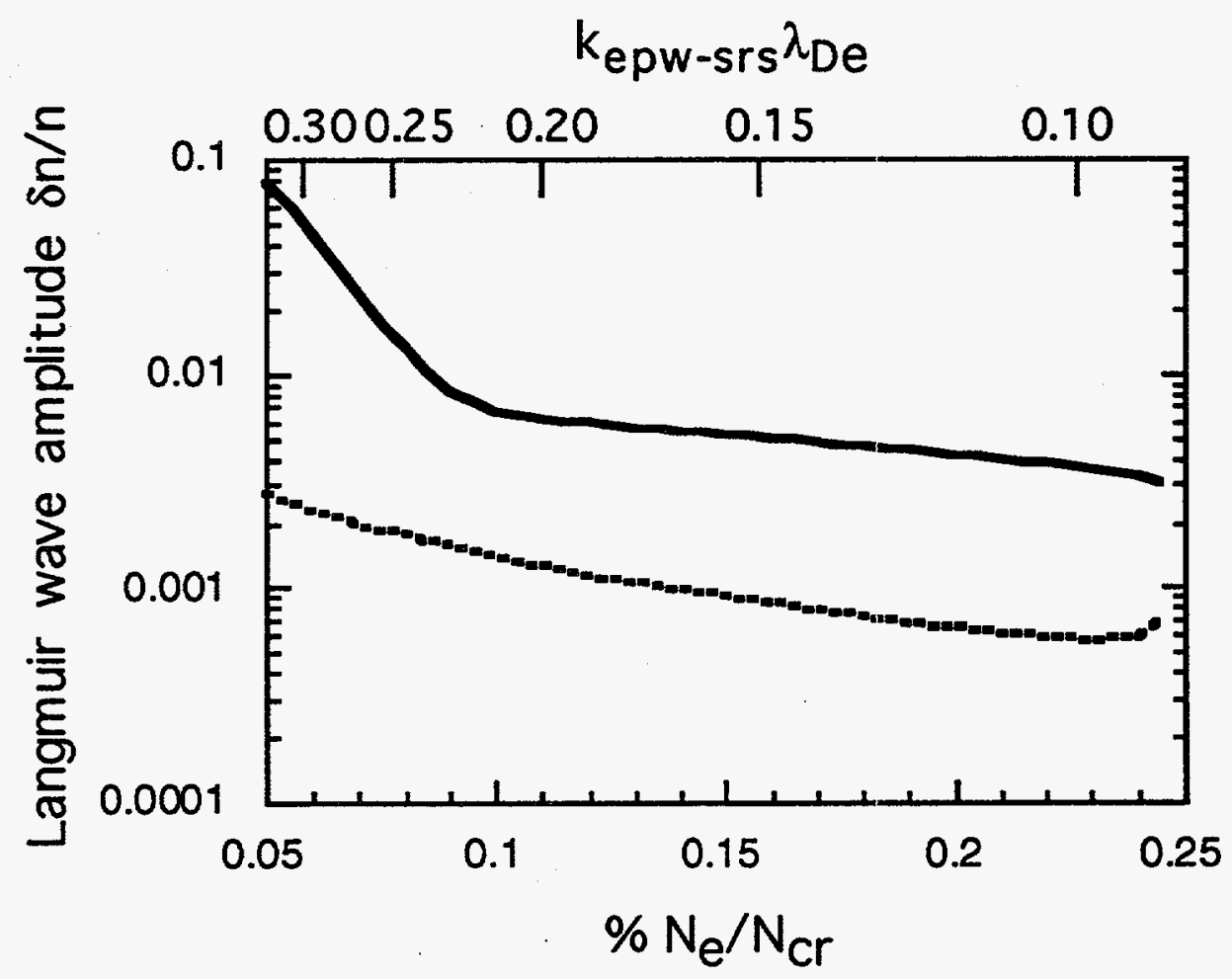

Figure 2.7.1 Comparison of the Langmuir wave amplitude at saturation predicted by the ponderomotive detuning model(dashed line) versus the Langmuir wave amplitude at threshold for the Langmuir decay instability(solid line).

problem is found by examining the linear version of equation 2.7.1 above. The assumption is made that the wave quantities in equation 2.7 .1 can be written as a slowly varying spatial component multiplied by a fast oscillating component in time and space. This reduces the above equation to 


$$
\frac{d \Psi_{e}}{d x}=\frac{-i c^{2} k_{e p w}^{2}}{4 V_{e p w} \omega_{e p w}} \Psi_{o} \Psi_{s} \Pi\left(\frac{x}{2 x_{\text {int }}}\right)
$$

where $\Pi\left[0.5 x_{\text {int }}^{-1}\right]$ is unity between - $x_{i n t}$ and $x_{i n t}$ and zero elsewhere. $x_{i n t}$ represents the length over which the process is driven before saturation. The solution to equation 2.7.3 is simply $\Psi_{\mathrm{e}}=\left[-0.25 \mathrm{ic}^{2} \mathrm{k}_{\mathrm{epw}}^{2} \Psi_{\mathrm{o}} \Psi_{\mathrm{s}} \mathrm{V}_{\mathrm{epw}}^{-1} \omega_{\text {epw }}^{-1}\right] \mathrm{x}$. The resonance width is found by Fourier transforming equation 2.7.3 and looking for the FWHM in the same manner as described in section 2.3. The Fourier transform of equation 2.7 .3 is

$$
\Psi_{e}(k)=\frac{-c^{2} k_{e p w}^{2} x_{\text {int }}^{2}}{2 V_{\text {epw }} \omega_{\text {epw }}} \Psi_{o} \Psi_{s} \frac{\sin \left(k x_{\text {int }}\right)}{\left(k x_{\text {int }}\right)^{2}}
$$

It is apparent from equation 2.7.4 that the FWHM is proportional to the inverse of the interaction length or $\Delta \mathrm{k} \propto \mathrm{x}_{\mathrm{int}}^{-1}$. The interaction length is found by equating the resonance width, $\Delta \mathrm{k} \propto \mathrm{x}_{\mathrm{int}}^{-1}$, with the change in wavenumber, $\left.\Delta \mathrm{k} \propto \mathrm{n}_{\mathrm{epw}}\left(\mathrm{n}_{\mathrm{oe}}\right)^{-1}\right|^{2} \omega_{\mathrm{pe}}\left[\left(\mathrm{k}_{\mathrm{epw}}^{2} \lambda_{\mathrm{pe}}^{2}\right) \mathrm{V}_{\mathrm{epw}}\right]^{-1}$, found above. This yields an interaction length proportional to $\mathrm{x}_{\text {int }} \propto\left(\mathrm{k}_{\mathrm{epw}}^{2} \lambda_{\mathrm{pe}}^{2} \mathrm{~V}_{\mathrm{epw}} \omega_{\mathrm{pe}}^{-1}\right)^{1 / 3}\left(\mathrm{c}^{2} \mathrm{k}_{\mathrm{epw}}^{2} \Psi_{\mathrm{o}} \Psi_{\mathrm{s}} \mathrm{V}_{\mathrm{epw}}^{-1} \omega_{\mathrm{epw}}^{-1}\right)^{-2 / 3}$. The saturation amplitude of the Langmuir wave is then

$$
\frac{\mathrm{n}_{\mathrm{epw}}}{\mathrm{n}_{\mathrm{oe}}} \propto\left(\mathrm{k}_{\mathrm{epw}} \lambda_{\mathrm{oe}}\right)^{2 / 3}\left(\mathrm{c}^{2} \mathrm{k}_{\mathrm{epw}}^{2} \Psi_{\mathrm{o}} \Psi_{\mathrm{s}} \omega_{\mathrm{epw}}^{-2}\right)^{1 / 3}
$$


It should be noted that this is lower than the value predicted by relativistic detuning for the beat wave process described below in section 2.9.

\subsection{Mode Coupling(unstimulated processes)}

\section{a. Self-interaction between different decay triangles}

The wave-wave saturation of stimulated Raman scattering has primarily dealt with the Langmuir decay instability, whereby the Langmuir wave decays into a secondary Langmuir wave and an ion acoustic wave. This section focuses on the effect of mode coupling between several decay triangles associated with stimulated Raman scattering. In this manner, energy is fed into both Langmuir waves directly from the stimulated Raman process itself. This process has been observed in the case of two plasmon decay through particle-in-cell simulations[53], as well as Thomson scattering experiments described below[54]. The ponderomotive force created by the coupling of these two Langmuir waves then drives an ion acoustic wave. Unlike the stimulated process of the Langmuir decay instability, mode coupling has no threshold to overcome, assuming stimulated Raman scattering is being driven.

When the Langmuir waves, associated with separate decay triangles of stimulated Raman scattering, drive the ion acoustic wave, the ion acoustic wave experiences secular growth. Because of the ion wave's relatively high ratio of damping to ion acoustic frequency, the amplitude that the ion acoustic waves are driven is determined primarily by damping rather than 
plasma inhomogeneity. An ion acoustic wave is driven due to the ponderomotive pressure of two Langmuir waves as shown by

$$
\begin{aligned}
& {\left[\left(\frac{\partial}{\partial t}-\overrightarrow{\mathrm{u}}_{\mathrm{f}} \cdot \frac{\partial}{\partial \mathrm{x}}\right)^{2}+2 v \frac{\partial}{\partial t}-\mathrm{c}_{\mathrm{s}}^{2} \frac{\partial^{2}}{\partial \mathrm{x}^{2}}\right] \frac{\mathrm{n}_{\mathrm{i}}}{\mathrm{n}_{\mathrm{oe}}}=} \\
& \frac{\mathrm{Zm}_{\mathrm{e}}}{\mathrm{M}_{\mathrm{i}}} \frac{\omega_{\mathrm{epw} 1} \omega_{\mathrm{epw} 2}}{\mathrm{k}_{\mathrm{epw} 1}^{2} \mathrm{k}_{\mathrm{epw} 2}^{2}}\left(\overrightarrow{\mathrm{k}}_{\mathrm{epw} 1} \cdot \overrightarrow{\mathrm{k}}_{\mathrm{epw} 2}\right) \frac{\partial^{2}}{\partial \mathrm{x}^{2}}\left(\frac{\mathrm{n}_{\mathrm{epw} 1}}{\mathrm{n}_{\mathrm{oe}}} \frac{\mathrm{n}_{\mathrm{epw} 2}}{\mathrm{n}_{\mathrm{oe}}}\right),
\end{aligned}
$$

where $\mathbf{Z}$ is the average charge state in the plasma, $\mathrm{m}_{\mathbf{e}}$ is the electron mass, $\mathrm{Mi}_{\mathbf{i}}$ is the ion mass, $\omega_{e p w 1}$ and $\omega_{e p w} 2$ are the Langmuir pump frequencies, kepw1 and kepw 2 are the Langmuir pump wavenumbers, nepw1/noe and $n_{\text {epw } 2 / n_{0 e}}$ are the pump Langmuir wave amplitudes, $c_{S}$ is the sound speed, and $n_{i a} / n_{o e}$ is the amplitude of the ion acoustic wave. The wave amplitudes are assumed to be of the form $\frac{\mathrm{n}_{\alpha}}{\mathrm{n}_{\mathrm{oe}}}=\frac{1}{2} \Psi_{\alpha} \exp \left[\mathrm{i}\left(\int \overrightarrow{\mathrm{k}}_{\alpha} \cdot \mathrm{d} \overrightarrow{\mathrm{x}}-\omega_{\alpha} \mathrm{t}\right)\right]+$ c.c.. For a resonant interaction, equation

2.8.1 can then be written in the form

$$
\begin{aligned}
& \left|\frac{\mathrm{n}_{\mathrm{ia}}}{\mathrm{n}_{\mathrm{oe}}}\right|^{2}=\left(\frac{\mathrm{Zm} \mathrm{e}}{\mathrm{M}_{\mathrm{i}}} \frac{\omega_{\mathrm{epw1}} \omega_{\mathrm{epw} 2} \mathrm{k}_{\mathrm{ia}}^{2}}{\mathrm{k}_{\mathrm{epw} 1} \mathrm{k}_{\mathrm{epw} 2}}\left(\hat{\mathrm{k}}_{\mathrm{epw} 1} \cdot \hat{\mathrm{k}}_{\mathrm{epw} 2}\right)\right)^{2} \\
& \left|\frac{\mathrm{n}_{\mathrm{epw} 1}}{\mathrm{n}_{\mathrm{oe}}}\right|^{2}\left|\frac{\mathrm{n}_{\mathrm{epw} 2}}{\mathrm{n}_{\mathrm{oe}}}\right|^{2}\left(\left(\left(\omega-\overrightarrow{\mathrm{k}}_{\mathrm{ia}} \cdot \overrightarrow{\mathrm{u}}_{\mathrm{f}}\right)^{2}-c_{\mathrm{s}}^{2} \mathrm{k}_{\mathrm{ia}}^{2}\right)^{2}+4 \omega_{\mathrm{ia}}^{2} v^{2}\right)^{-1} .
\end{aligned}
$$

It is interesting to note that mode coupling between these two decay triangles associated with stimulated Raman scattering produces long wavelength ion acoustic fluctuations. These fluctuations are close to the 
wavelength which Nicholson[25] found to be capable of causing absolute instability. Nicholson investigated the effect of a time-independent sinusoidal density perturbation superimposed on a linear density gradient. He found that the sinusoidal density perturbation could induce an absolute instability and that this effect was strongest when $\lambda_{\text {ion }} \approx 2 \pi \sqrt{V_{2} V_{1}} \gamma_{o}^{-1}$. This introduces the very interesting possibility that mode coupling between the different decay triangles driven by stimulated Raman scattering could induce absolute instability. This would then provide an explanation for the large levels of Raman seen in experiments in which the wavenumber mismatch is strictly linear and the Raman process should be a convective instability. There have been many experiments which have seen considerably larger levels of stimulated Raman scattering than would be predicted from the expected convective gain[55]. This gain is difficult to explain even invoking filamentation as a means of enhancing the local laser intensity. These ion waves could also induce an absolute instability for stimulated Brillouin scattering and thereby provide a mechanism whereby stimulated Raman scattering could indirectly produce large levels of stimulated Brillouin scattering. The apparent seeding of stimulated Brillouin scattering has been previously observed in Thomson scattering experinnents[56]. Mode coupling could also play a critical role in the saturation of the instability as well due to the modification of the background density by the ion acoustic waves. This detuning mechanism is discussed in section $2.8 \mathrm{~b}$.

A Thomson scattering experiment would be an ideal way of looking for the ion waves which would be produced by this mode-coupling process. The ion waves produced in this experiment would have very small 
wavenumbers resulting in small angle Thomson scattered signals. If the experiment were done with a thin exploding foil target, a $526 \mathrm{~nm}$ interaction beam could be used to drive the stimulated Raman scattering which would make it easily diagnosed with a streak camera. A $1 \mu \mathrm{m}$ probe beam could then be used which would result in a much larger angle scattering due to the smaller disparity between the probe wavenumber and the ion acoustic wavenumber. The primary concern would be in ensuring that the quartercritical surface is not present for the $526 \mathrm{~nm}$ interaction beam which would produce large levels of $1 \mu \mathrm{m}$ light from $\omega_{\mathrm{o}} / 2$ emission at the quarter-critical surface.

The growth of the ion waves and their effect on the Raman process could be looked at in detail using fluid codes in which the feedback process for the Langmuir decay instability and the modulational instability has been eliminated by taking out the coupling term in the Langmuir wave equation, representing the coupling between the ion waves and the Langmuir waves. With modifications, a code such as SATIN could be used for such a study[57]. The equations describing stimulated Raman scattering along with an ion wave equation driven by the Raman produced Langmuir waves could be solved in 2-D with a linear inhomogeneity to investigate the possibility that Raman could be driven absolute in a linear density gradient due to the ion fluctuations driven by the mode coupling of Langmuir waves from different decay triangles. The pressure associated with the ion waves would act on the plasma allowing the ion waves to alter the local plasma density so that the equations describing the Raman process see a plasma density modified by the ion wave fluctuations. This code would then allow the study of stimulated 
Raman scattering to see if absolute instability could ensue. The ion waves acting on the plasma density could affect the saturation of stimulated Raman scattering by modifying the background density. This then provides a means by which stimulated Raman scattering can be driven absolute in a linear density gradient thereby explaining the large levels of Raman seen in experiments. It also provides a means whereby the Raman process can be saturated, possibly at a lower value than that predicted by the Langmuir waves overcoming the damping threshold for the Langmuir decay instability. In any case, mode coupling is very important in determining the level at which the Langmuir and ion acoustic waves grow. It is also very important for determining what particular decay geometry of LDI wins, more so than which has the highest growth rate which is demonstrated below.

One of the clearest experimental evidence for mode coupling involved a Thomson scattering experiment with two plasmon decay[54]. Many of the first Thomson scattering experiments used $\mathrm{CO}_{2}$ lasers to interact with the plasma and drive Two Plasmon Decay[58]. These experiments utilized visible light to perform Thomson scattering off of the Langmuir waves and subsequent ion waves produced in Two Plasmon Decay. In one of these experiments, the simultaneous spectrum of Langmuir waves and ion acoustic waves were measured[54]. The experimentally measured wavenumber spectrum of electrostatic waves is shown in figure 2.8.1.

The magnitude of the wavenumbers driven in two plasmon decay are very sensitive to the electron temperature of the plasma. Many of the $\mathrm{CO}_{2}$ experiments had electron temperatures of less than $100 \mathrm{eV}$. As a consequence the Langmuir waves excited had wavenumber magnitudes much greater 
than the wavenumber of the incident pump. Because these wavenumbers were so much larger than the incident wavenumber, the magnitude of the

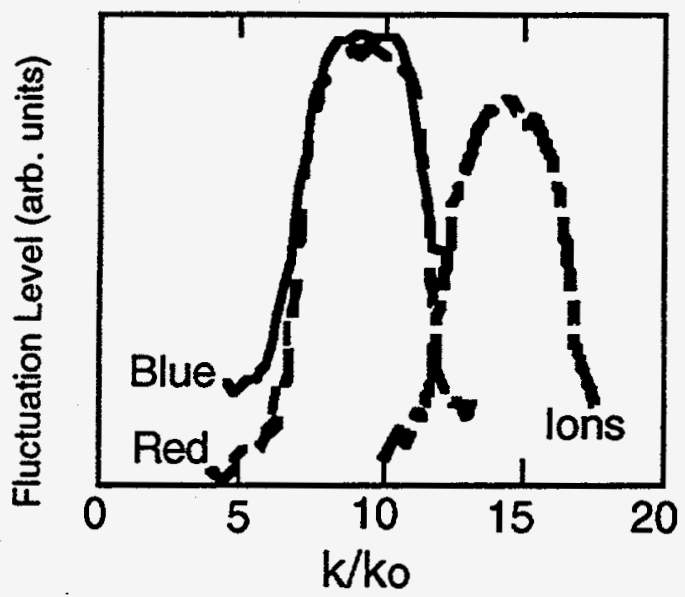

Figure 2.8.1 Wavenumber resolved Thomson scatter spectrum from Langmuir waves and ion waves participating in stimulated Raman scattering. This graph has been reprinted from Baldis et al. 1991, [17].

Langmuir wavenumbers are insensitive to their angle relative to the incident wavenumber. The wavenumbers are then sensitive primarily to the density and temperature of the plasma. In the experiment shown above the wavenumbers are roughly between 7 and 13 times the wavenumber of the incident electromagnetic wavenumber. Assuming the long wavelength cutoff of the waves correspond to a value of $k \lambda_{\mathrm{De}}=0.3$, the electron temperature is estimated at approximately $75 \mathrm{eV}$. If the long wavenumber cutoff is chosen to occur at a value of $k \lambda_{D e}=0.3$, then the short wavenumber cutoff can be estimated from the Thomson scattered data as approximately $k \lambda_{D e}=0.15$. It is 
interesting to note that this spectrum appears to have a gap for small $k \lambda_{D e}$ values, very similar to stimulated Raman scattering.

There are several mechanisms which can lead to the generation of ion acoustic waves. These mechanisms include the Langmuir decay instability in which a Langmuir wave decays into a Langmuir wave and an ion acoustic wave, the electromagnetic decay instability, the coupling of Langmuir waves to drive ion acoustic waves, and the modulational instability which is a four wave process. The ion waves shown in the data above have wavenumbers greater than the wavenumber belonging to the waves which precludes the modulational instability which drives near zero wavenumber ion waves. Likewise, the electromagnetic decay instability, described in chapter 3 , produces ion acoustic waves with wavenumbers approximately equal to the wavenumber of the Langmuir waves, which was not observed in the experiment. Because the scattering process(mode coupling) has no threshold to overcome, it is likely that this process will be the first to occur.

In the scattering process, Langmuir waves from different decay triangles couple together to drive ion acoustic waves. For this scattering process to be resonant, it must satisfy the frequency and wavenumber matching conditions $\omega_{\mathrm{ia}}=\omega_{\mathrm{epw} 1}+\omega_{\mathrm{epw} 3}$ and $\overrightarrow{\mathrm{k}}_{\mathrm{ia}}=\overrightarrow{\mathrm{k}}_{\mathrm{epw} 1}+\overrightarrow{\mathrm{k}}_{\mathrm{epw} 3}$ where $\omega_{\mathrm{ia}}\left(\overrightarrow{\mathrm{k}}_{\mathrm{ia}}\right), \omega_{\mathrm{epw} 1}\left(\overrightarrow{\mathrm{k}}_{\mathrm{epw1}}\right)$, and $\omega_{\mathrm{epw} 3}\left(\overrightarrow{\mathrm{k}}_{\mathrm{epw} 3}\right)$ are the frequency(wavenumber) for the scattered ion acoustic wave, the first Langmuir wave, and the third Langmuir wave respectively. The two Langmuir waves are from different decay triangles. The waves can possibly satisfy the matching conditions between an ion acoustic wave and both a red plasmon and a blue plasmon. The coupling of a blue(red) plasmon with another blue(red) plasmon 
produces smaller wavenumber ion acoustic waves and the coupling of a blue plasmon with a red plasmon produces ion acoustic waves with larger wavenumbers.

For the parameters of this experiment, the coupling between red and blue plasmons produce ion acoustic wavenumbers which vary from $1.5 \mathrm{kepw}$ near the higher wavenumber cutoff of the spectrum to approximately 1.56 at the lower wavenumber cutoff of the spectra. The magnitude of the ion wavenumber generated with this coupling is very insensitive to both angle and density. These wavenumber magnitudes agree extremely well with the Thomson scattered data shown in figure 2.8.1, which is in contrast with the analysis of the authors who assumed that the ion acoustic wavenumber should be at twice the wavenumber of the Langmuir wave, which is the ion wavenumber with the highest growth rate involved in the Langmuir decay instability. The shorter ion acoustic wavenumber, however, varies considerably with angle but is insensitive to density. In this case, to generate the shorter wavenumber ion acoustic fluctuation, a red(blue) plasmon at some angle to the incident wavenumber has to couple with a red(blue) plasmon scattered nearly perpendicular to the incident laser wavenumber. The absence of the shorter wavenumber ion acoustic waves is then likely due to the absence of the appropriate waves to couple to. When the two large amplitude Langmuir waves drive the ion acoustic wave, the ion acoustic wave experiences secular growth.

Another interesting possibility is the coupling between Langmuir waves associated with two plasmon decay to drive an electromagnetic wave. This mechanism is identical to a mechanism operating at the critical surface 
which produces second harmonic emission. I first submitted an experimental proposal to study this mechanism at the quarter critical surface to the University of Rochester in 1992. This process was studied independently by Russel et al.[59] using numerical simulations. In this process, Langmuir waves from different decay triangles associated with two plasmon decay couple together driving an electromagnetic wave which satisfies the wavenumber and frequency matching conditions

$$
\begin{aligned}
& \omega_{\text {epw } 1}+\omega_{\text {epw } 2}=\omega_{\mathrm{s}} \text { and } \\
& \overrightarrow{\mathrm{k}}_{\mathrm{epw} 1}+\overrightarrow{\mathrm{k}}_{\mathrm{epw} 2}=\overrightarrow{\mathrm{k}}_{\mathrm{s}}
\end{aligned}
$$

where $\omega_{\text {epw } 1}\left(k_{e p w 1}\right), \omega_{\text {epw }}\left(k_{\text {epw }}\right)$, and $\omega_{S}\left(k_{S}\right)$ are the frequency (wavenumber) of the first Langmuir wave, the second Langmuir wave, and the driven electromagnetic wave respectively. The equation describing the amplitude of the electromagnetic wave produced by the coupling of two Langmuir waves is

$$
\left(\frac{\partial^{2}}{\partial t^{2}}+\omega_{p e}^{2}-c^{2} \nabla^{2}\right) \frac{q A_{s}}{m_{e} c^{2}}=\omega_{p e}^{2}\left(\frac{n_{e p w 1}}{n_{o e}} \frac{u_{e p w 2}}{c}\right)_{\text {transverse }}
$$

where $\mathrm{qA}_{\mathrm{s}} / \mathrm{mec}^{2}, \mathrm{n}_{\mathrm{epw}} / \mathrm{n}_{\mathrm{oe}}, \mathrm{u}_{\mathrm{epw}} / \mathrm{c}$ is the amplitude of the electromagnetic wave, the amplitude of the first Langmuir wave, and the oscillation velocity of the electrons in the second Langmuir wave, respectively. Using the procedure outlined in chapter 4 , the amplitude of the electromagnetic wave produced in a linear profile will be 


$$
\left|\Psi_{s}\right|=\frac{\sqrt{2 \pi}}{4} \frac{\omega_{\mathrm{pe}}^{2}}{\omega_{s} v_{\mathrm{gs}} \sqrt{\kappa^{\prime}}}\left|\Psi_{\text {epw1 } 1}\right|\left|\Psi_{\text {epw } 2}\right|
$$

The process of driving the electromagnetic wave takes energy out of the Langmuir waves providing a damping mechanism which can help to saturate the instability. The electromagnetic wave can take a significant amount of energy out of the Langmuir waves, while the ion wave discussed above does not take a significant amount of energy from the waves but rather acts to transfer energy between the different decay triangles. The large level ion wave can, however, modify the background density and detune the parametric instabilities, as well as cause profile steepening. Another of the important possibilities for this mechanism is that the electromagnetic wave produced in this process could act as an enhanced noise source from which stimulated Brillouin scattering could grow. Stimulated Brillouin scattering is a parametric instability in which an electromagnetic wave drives a scattered electromagnetic wave and an ion acoustic wave.

An experiment to look for this scattering process will be plagued by the need to eliminate the occurrence of stimulated Brillouin scattering. An experiment could involve using a gas bag target or capillary discharge which would be filled with a mixture of Helium and Hydrogen which results in large damping of the ion acoustic wave driven by stimulated Brillouin scattering. A plot of the damping as a function of the ratio of the ion temperature to the electron temperature can be seen in figure 4.4 .5 in Appendix 4.4. Secondly, the scattered light would be collected which had polarization rotated 90 degrees from the interaction beam which should 
greatly reduce the effects of stimulated Brillouin scattering whose gain exponent is proportional to the square of the cosine of the polarization angle between the incident and scattered light. The gas fill in the targets could be varied from below around ten percent of critical to above quarter critical to look for a change in the electromagnetic emission near the laser frequency as the two plasmon decay is turned on when densities around twenty to twenty five percent are present. Another possibility would be to use an exploding foil target to let the peak density change from above quarter critical to below twenty percent of critical while monitoring the level of "SBS like" emission which was the original design of the experiment submitted to Rochester, however, the enhanced damping obtainable with gas mixtures should help to reduce the stimulated Brillouin scattering signal.

\section{b. Interaction between different instabilities}

The most widely studied interaction between different instabilities involves the interaction between stimulated Raman scattering and stimulated Brillouin scattering[11,56,60,61]. One way stimulated Brillouin scattering can saturate the Raman process is through quasi-resonant mode coupling[4,61-63]. In this process a Langmuir wave can couple with an ion wave generating Langmuir waves which have a wavevector, $\mathrm{k}_{\mathrm{L}}$, equal to the original Langmuir wavevector, $k_{e p w}$, plus an integer number of the ion wave's wavevector or $\vec{k}_{\mathrm{L}}=\vec{k}_{\text {epw }} \pm n k_{\text {ion }} \hat{x}$. For this process to be efficient the resultant mismatch between the Langmuir waves must be less than the frequency variation, $\Delta \omega$, produced by the ion wave, $\Delta \omega / \omega_{\mathrm{pe}}=0.5 \mathrm{n}_{\mathrm{ia}} / \mathrm{n}_{\mathrm{oi}}[62]$. 
Therefore, this quasi-resonant mode coupling typically requires large amplitude ion acoustic waves before this process becomes efficient. The source of ion waves typically proposed for this process is stimulated Brillouin scattering[64]. If ion waves are produced which satisfy the above criteria, then energy may be efficiently transferred from the Raman driven Langmuir wave into secondary Langmuir waves. The generation of these coupled modes can introduce a large nonlinear damping into the original Raman process thereby saturating stimulated Raman scattering[4]. As pointed out above in 2.8 a, the Langmuir waves from different decay triangles can couple together driving ion waves which could then undergo quasi-resonant mode coupling if the ion waves were driven to sufficient amplitude.

Stimulated Brillouin scattering can also saturate stimulated Raman scattering due to the detuning effects associated with the modifications of the background density profile caused by the ion waves[11,65]. The Langmuir wave equation for stimulated Raman scattering is modified by the density fluctuation as shown in equation 2.7.6 where the low frequency fluctuation $\mathrm{n}_{\mathrm{el}} / \mathrm{n}_{\mathrm{oe}}$ represents the ion wave. Stimulated Raman scattering is detuned when the wavenumber change induced by the density fluctuation becomes larger than the resonance width of the instability shown above to be proportional to $\Delta \mathrm{k} \propto \gamma_{\mathrm{o}}\left(\mathrm{V}_{\mathrm{epw}} \mathrm{V}_{\mathrm{s}}\right)^{-0.5}$. The change in wavenumber induced by the ion wave is given by $\Delta \mathrm{k} \propto\left(\mathrm{n}_{\mathrm{el}} / \mathrm{n}_{\mathrm{oe}}\right)\left(\mathrm{k}_{\mathrm{epw}} \lambda_{\mathrm{De}}^{2}\right)^{-0.5}$. The Raman process can then become detuned when the ion wave amplitude, $n_{e l} / n_{o e}$, has reached a value $\left(\mathrm{n}_{\mathrm{el}} / \mathrm{n}_{\mathrm{oe}}\right) \propto\left(\mathrm{v}_{\mathrm{os}} / \mathrm{c}\right) \mathrm{k}_{\mathrm{epw}} \lambda_{\mathrm{De}} \sqrt{\mathrm{k}_{\mathrm{epw}} \mathrm{k}_{\mathrm{s}}^{-1}}$. A distribution of ion waves, however, will likely reduce the stabilization effect on the Raman process. These ion waves could be produced by a number of sources. As pointed out 
above in 2.8 a, the Langmuir waves from different decay triangles can couple together driving ion waves which could lead to the detuning of the Raman process. Also the Langmuir waves could become large enough to form cavitons as discussed in section 2.6[11]. The density fluctuations resulting from these cavitons can then produce enhanced levels of ion waves which can both seed stimulated Brillouin scattering and reduce the level of stimulated Raman scattering[11]. Stimulated Brillouin scattering could produce large levels of ion waves which could detune the Raman process $[60,65,66]$. Forward Brillouin scatter can also be seeded by two crossed laser beams. Eventually this technique could be used in laser-driven fusion to control stimulated Raman scattering through the generation of large amplitude ion waves. These ion waves would take a minimal amount of energy from the driver beams with all but a very small fraction of the energy going into the forward direction.

In many experiments, a gap in the spectrum of stimulated Raman scattering is observed[39]. This gap typically ranges from slightly below $\mathrm{n}_{\mathrm{e}} / \mathrm{n}_{\mathrm{cr}}=0.25$ to approximately $\mathrm{n}_{\mathrm{e}} / \mathrm{n}_{\mathrm{cr}}=0.2$. This is also the range of densities over which two plasmon decay is driven. As discussed in 2.8 a above, the Langmuir waves associated with two plasmon decay can couple together driving large amplitude ion acoustic waves[53,54]. In addition, the Langmuir waves driven by two plasmon decay can undergo decay by several of the secondary processes discussed in section 2.5 driving large amplitude Langmuir waves. Additionally the Langmuir waves can undergo collapse as discussed in section 2.6 leading to the generation of ion fluctuations. These ion waves can then detune the Raman process as discussed above. This 
represents one possible explanation of the Raman gap although there are several other explanations $[39,67,68]$. The relation given above for the detuning by ion waves, $\left(\mathrm{n}_{\mathrm{el}} / \mathrm{n}_{\mathrm{oe}}\right) \propto\left(\mathrm{v}_{\mathrm{os}} / \mathrm{c}\right) \mathrm{k}_{\mathrm{epw}} \lambda_{\mathrm{De}} \sqrt{\mathrm{k}_{\mathrm{epw}} \mathrm{k}_{\mathrm{s}}^{-1}}$, suggests that the gap should begin to disappear when the temperature of the plasma is increased sufficiently, which consequently raises the value of $k_{e p w} \lambda_{\text {De }}$ in the gap region. An interesting experiment to look at the effects of the ion dependent saturation mechanisms on the gap would involve looking at stimulated Raman scattering from a solid target utilizing a high-intensity short-pulse laser. If stimulated Raman scattering is driven hard enough that it grows faster than the ion waves can saturate the instability, then the gap should not be present in the case of these high-intensity short-pulse experiments. In addition, the high $v_{o s} / c$ should reduce the stabilizing effect of the ion waves.

\subsection{Relativistic detuning}

This section examines relativistic effects on the detuning of parametric instabilities involving waves. Relativistic detuning become important when the oscillation velocity of the electrons become relativistic. Recent short pulse experiments have achieved high enough intensities that the oscillation velocity of the electrons in the electromagnetic field is relativistic. Likewise there are conditions where the oscillation velocity of the electrons can become relativistic due to the electrostatic field of the Langmuir wave itself. The continuity equation, 


$$
\frac{\partial \mathrm{n}}{\partial t}+\nabla \cdot(\mathrm{n} \overrightarrow{\mathrm{u}})=0
$$

provides insight into where relativistic effects are important in the latter case. By assuming a plane wave solution and linearizing the density into zero order and first order components, the continuity equation can be written as

$$
\frac{\mathrm{u}_{\mathrm{os}}}{\mathrm{c}} \approx \frac{\omega_{\mathrm{epw}}}{\mathrm{ck}_{\mathrm{epw}}} \frac{\delta \mathrm{n}}{\mathrm{n}_{\mathrm{oe}}}
$$

This shows that the oscillation velocity is relativistic primarily for Langmuir waves with very small wavenumbers. Therefore, the instabilities most affected by this detuning mechanism should be the oscillating two stream instability at the critical surface and to a lesser degree forward stimulated Raman scattering.

The saturation of waves by relativistic detuning was first considered by Rosenbluth and Lui[7]. The specific case which they considered was a Langmuir wave driven by two electromagnetic waves, which is unstimulated Raman forward scattering. Because the oscillation velocity of the electrons is proportional to the amplitude of the electron plasma wave, as the wave grows the oscillation velocity of the electrons changes causing the frequency of the wave to change and the scattering process to eventually become detuned. For stimulated processes, the frequency resonance width of the instability is proportional to the growth rate so that when the Langmuir wave's frequency changes by an amount equal to some number of growth 
rates of the instability, the resonance width, the instability detunes itself and does not experience further growth.

Recently, the case of stimulated Raman forward scattering has been looked at[69]. For stimulated Raman forward scatter, the wavenumber of the wave is approximately $\mathrm{kepw}_{\mathrm{ep}}=\omega_{\mathrm{pe}} / \mathrm{c}$. This calculation is detailed in Appendix 2.7. The equation describing the growth of the Langmuir wave participating in Raman scattering may be written as

$$
\begin{aligned}
& \frac{\ddot{\zeta}}{\left[1-(\dot{\zeta} / \mathrm{c})^{2}\right]^{1.5}}+\left(\omega_{\mathrm{pe}}^{2}-3 \mathrm{v}_{\mathrm{th}}^{2} \nabla^{2}\right) \zeta= \\
& \frac{-\mathrm{q}^{2}}{\mathrm{~m}_{\mathrm{e}}^{2} \mathrm{c}^{2}} \sqrt{1-(\dot{\zeta} / \mathrm{c})^{2}} \nabla\left(\overrightarrow{\mathrm{A}}_{\text {scatt }} \cdot \overrightarrow{\mathrm{A}}_{\text {pump }}\right),
\end{aligned}
$$

where the Langmuir wave amplitude, $n_{1 e} / n_{o e}$, is equal to $n_{1 e} / n_{o e}=|\nabla \zeta|$. The left hand side of the above equation can be expressed roughly by the following dispersion relation

$$
\omega_{\mathrm{epw}}^{2}\left[1+1.5(\dot{\zeta} / \mathrm{c})^{2}\right]+\omega_{\mathrm{pe}}^{2}+3 \mathrm{v}_{\mathrm{th}}^{2} \mathrm{k}_{\mathrm{epw}}^{2} \approx 0
$$

with the assumption that when $(\dot{\zeta} / \mathrm{c})$ is small, $\omega_{\mathrm{epw}}^{2}+\omega_{\mathrm{pe}}^{2}+3 \mathrm{v}_{\mathrm{th}}^{2} \mathrm{k}_{\mathrm{epw}}^{2} \approx 0$ is satisfied. As the Langmuir wave grows in amplitude, the wave's frequency changes, $\omega_{\text {epw }} \Rightarrow \omega_{\text {epw }}+\delta$. By expanding the dispersion relation, this change in frequency is found to be proportional to $\delta \propto \omega_{\text {pe }}(\dot{\zeta} / \mathrm{c})^{2}$. Once the frequency changes by a number of growth rates, the instability is detuned and ceases to grow. The Langmuir wave amplitude at saturation is then proportional to 
$\mathrm{n}_{1 \mathrm{e}} / \mathrm{n}_{\mathrm{oe}} \propto\left(\mathrm{k}_{\mathrm{epw}} \mathrm{c} \omega_{\mathrm{pe}}^{-1}\right) \sqrt{\gamma_{\mathrm{o}} \omega_{\mathrm{pe}}^{-1}}$, which is similar, although higher, to the saturation amplitude for ponderomotive detuning discussed above. The numerical coefficient in front is approximately 2 as shown in appendix 2.6.

Equation 2.9.4 above is the same approximate "dispersion relation" used to describe the scattering process whereby two fixed amplitude lasers are used to drive a Langmuir wave. This scattering process, however, has a different resonance width. The resonance width for the scattering process is proportional to the inverse time over which the process grows, $\delta \propto \mathrm{t}_{\mathrm{int}}^{-1}$, as described in section 2.7 above. From the non-relativistic form of equation 2.9.3 above, the spatial fluid displacement at early times grows at the homogeneous growth rate, $\zeta \propto \beta$ t where $\beta=c^{2} k_{\text {epw }} \Psi_{\mathrm{o}} \Psi_{\mathrm{s}} \omega_{\mathrm{epw}}^{-1}$. Therefore, by combining the resonance width, $\delta \propto \mathrm{t}_{\text {int }}^{-1}$, of the scattering process with the frequency mismatch, $\delta \propto \omega_{\mathrm{pe}}\left(\omega_{\mathrm{epw}} \zeta c^{-1}\right)^{2}$, caused by the growing Langmuir wave and the amplitude of the fluid displacement itself, $\zeta \propto \beta t_{\text {int }}$, the interaction time is found to be proportional to $t_{\text {int }} \propto\left(c^{2} \omega_{\mathrm{pe}}^{-3}\right)^{1 / 3} \beta^{-2 / 3}$. The saturation amplitude which the wave grows is found simply by plugging the interaction time into the homogeneous growth rate. This results in a wave amplitude which is proportional to $\mathrm{n}_{\mathrm{epw}}\left(\mathrm{n}_{\mathrm{oe}}\right)^{-1} \propto\left(\mathrm{ck}_{\mathrm{epw}} \omega_{\mathrm{epw}}^{-1}\right)^{2 / 3}\left(\mathrm{c}^{2} \mathrm{k}_{\mathrm{epw}}^{2} \Psi_{\mathrm{o}} \Psi_{\mathrm{s}} \omega_{\mathrm{epw}}^{-2}\right)^{1 / 3}$ at saturation which is in agreement with previous results[7]. Relativistic detuning of the beatwave process predicts a higher saturation amplitude for the Langmuir waves by the factor $\left(\mathrm{cv}_{\mathrm{th}}^{-1}\right)^{2 / 3}$ over that predicted by the ponderomotive detuning of the beatwave process. It should be noted that both the relativistic detuning, as 
well as the ponderomotive detuning, neglect broadening of the frequency spectrum by the modulational instability.

\subsection{Particle trapping and wave breaking}

Another mechanism which can saturate the amplitude of a Langmuir wave is particle trapping. Particle trapping is a wave-particle interaction which leads to the saturation of parametric instabilities due to the induced nonlinear damping[16,70]. When particles in the plasma are traveling at approximately the phase velocity of a given electrostatic wave, they can be efficiently accelerated or decelerated by that wave. When the amplitude of the electrostatic wave becomes large, the electrostatic field of that wave can accelerate the thermal electrons in the plasma up to the phase velocity of the wave resulting in a large damping. The effects of particle trapping are best studied with particle-in-cell simulations or Vlasov simulations[16,70].

The effect of the finite amplitude of the Langmuir wave is to accelerate the background electrons closer to the phase velocity of the wave. This can be modeled roughly as an effective reduction in the phase velocity of the wave or consequently as an effective increase in the wavenumber, $k_{e f f}$, of the Langmuir wave. The effective increase in the wavenumber of the Langmuir wave can be approximated as $\mathrm{k}_{\text {eff }} \lambda_{\mathrm{De}}=\mathrm{k}_{\mathrm{epw}} \lambda_{\mathrm{De}}\left[1+\mathrm{k}_{\mathrm{epw}} \lambda_{\mathrm{De}}-\sqrt{\mathrm{k}_{\mathrm{epw}}^{2} \lambda_{\mathrm{De}}^{2}+2 \delta \mathrm{n} / \mathrm{n}}\right]^{-1}$. The Langmuir wave with its effective phase velocity is then treated as a small amplitude wave in which the analytic expression for the Landau damping is valid. The next step 
is to solve for the required value of $k_{e f f} \lambda_{D e}$ such that Landau damping is large enough to cause the instability to go below the convective damping threshold. Once this effective phase velocity of the wave is found, the amplitude of the Langmuir wave can be solved for using the above relation for the effective wavenumber of the Langmuir wave in terms of the actual wavenumber and the Langmuir wave amplitude. As an example, two plasmon decay is driven above the convective threshold discussed in section 2.2 when the growth rate is larger than the square root of the product of the damping of the two daughter waves which in the case of two plasmon decay is approximately $\gamma_{0}>v_{e p w}$. In the case of Langmuir waves, the analytic expression for the effective Landau damping of the Langmuir wave is given by $\omega_{\mathrm{i}} \omega_{\mathrm{epw}}^{-1} \approx \sqrt{\pi} \exp \left[-1.5-0.5\left(\mathrm{k}_{\mathrm{eff}} \lambda_{\mathrm{De}}\right)^{-2}\right]\left(2 \mathrm{k}_{\mathrm{eff}} \lambda_{\mathrm{De}}\right)^{-3}$ which is valid for $k_{\text {eff }} \lambda_{\mathrm{De}}<0.4$. The effective wavenumber can then be found in the case of two plasmon decay by equating the growth rate to the effective Landau damping and solving for $k_{\text {eff }} \lambda_{\mathrm{De}}$. This rough approximation can then be used to approximate the saturation amplitude of the Langmuir waves such that the damping induced by particle trapping causes the parametric instability to go below the convective damping threshold through the relation $\delta \mathrm{n} / \mathrm{n}=0.5\left[\left(1+\mathrm{k}_{\mathrm{epw}} \lambda_{\mathrm{De}}-\mathrm{k}_{\mathrm{epw}} \lambda_{\mathrm{De}} \mathrm{k}_{\mathrm{eff}}^{-1} \lambda_{\mathrm{De}}^{-1}\right)^{2}-\mathrm{k}_{\mathrm{epw}}^{2} \lambda_{\mathrm{De}}^{2}\right]$. As an example, for a typical irradiation intensity of $5 \times 10^{14} \mathrm{~W} / \mathrm{cm}^{2}$, the growth rate divided by the plasma frequency is approximately $9 \times 10^{-3}$. The corresponding value of $k_{\text {eff }} \lambda_{D e}$ such that the instability is below threshold is $k_{\text {eff }} \lambda_{D e}=0.28$. The resulting amplitude of the Langmuir waves at saturation is shown in figure 2.10.1 below as a function of the Langmuir waves actual $k_{e p w} \lambda_{D e}$. It is 
qualitatively clear that particle trapping predicts that the saturation amplitude of the Langmuir wave should increase with decreasing $k_{\text {epw }} \lambda_{\text {De }}$.

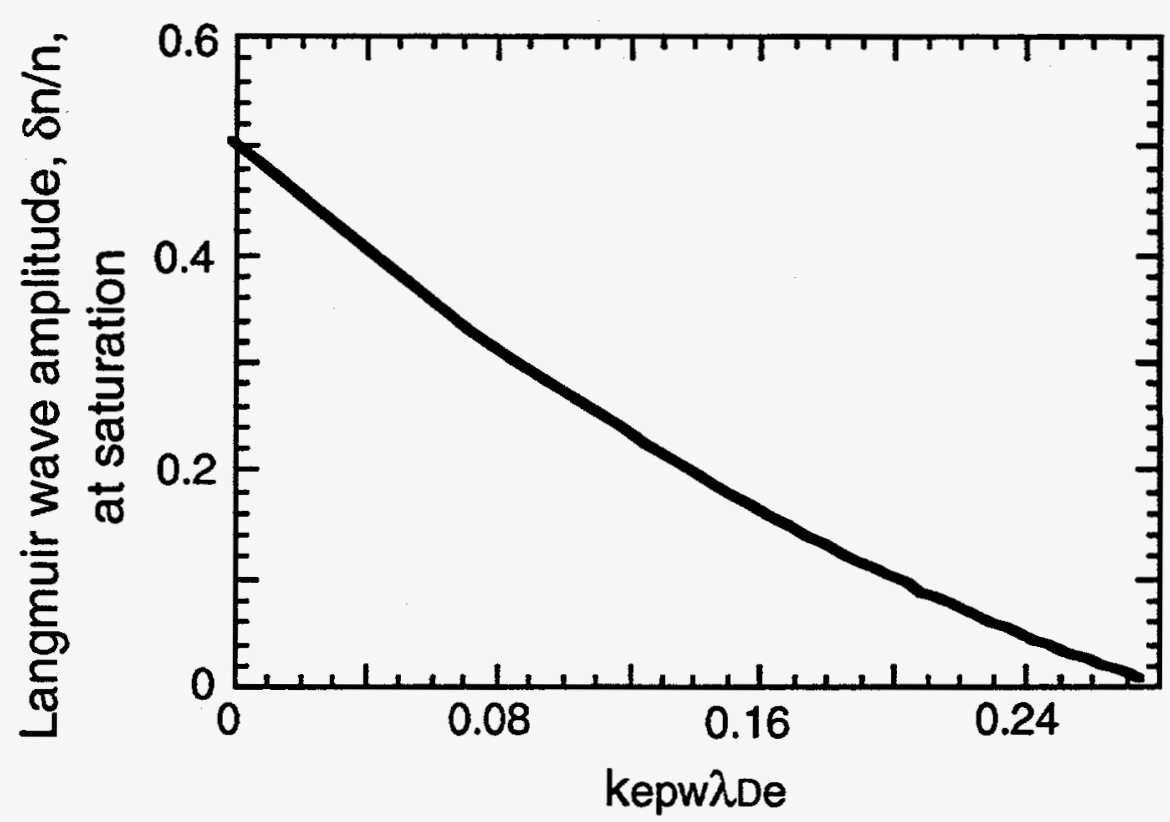

Figure 2.10.1 Approximate Langmuir wave amplitude for which two plasmon decay goes below the convective damping threshold as a function of the parameter kepw $\lambda_{\text {De }}$

Another possible saturation mechanism for Langmuir waves is wave breaking. In 1971, Coffey showed that a stationary Langmuir wave in a plasma could only exist in steady-state up to a fixed amplitude[71]. Figure 2.10.2 is the resulting graph from this paper showing the maximum amplitude a stationary Langmuir wave can reach in a plasma as a function of the product of the wavenumber of the Langmuir wave with the Debye length, $\lambda_{\mathrm{De}}$. The amplitude at the wave breaking limit is much greater than the threshold amplitude for secondary decay processes which can be seen by comparing 
figure 2.10 .2 below with figure 2.5 .1 in section 2.5 until $k \lambda_{\text {De }}$ becomes very large, greater than 0.5 .

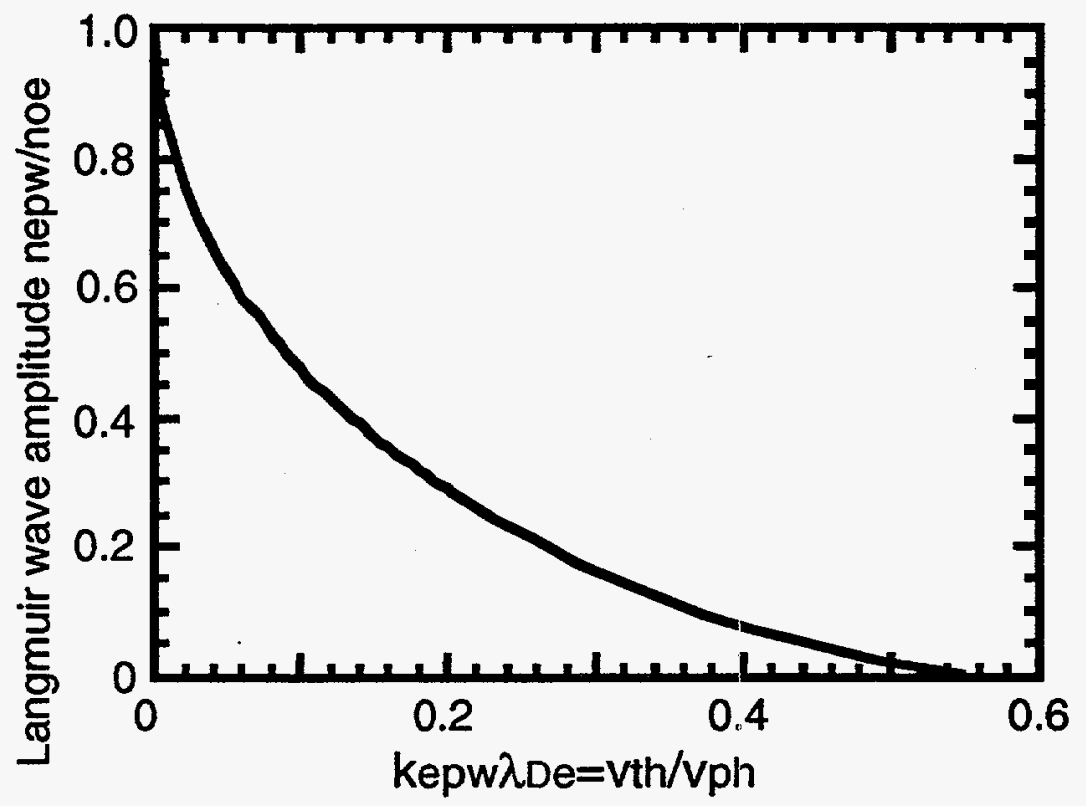

Figure 2.10.2 Plot showing the amplitude at which wave breaking becomes important for Langmuir waves. This plot was modified slightly from a paper by Coffey[71].

The main problem with this model, as well as particle trapping, is that it predicts that the saturation level of waves should increase with increasing phase velocity. Figure 2.10 .3 below shows a stimulated Raman spectrum taken from a solid target experiment[55]. This figure shows, as do most stimulated Raman spectra, that the stimulated Raman spectrum peaks where the phase velocity of the Langmuir waves is approximately three times the thermal velocity and decreases with increasing phase velocity. This scaling is in contrast to the predictions of either particle trapping or wave breaking. 
This is not too surprising considering the high amplitudes required of the Langmuir waves before these mechanisms become important. Particle-in-cell simulations typically see a reduction in the level of stimulated Raman scattering when the ions are mobile[72,73]. In the case of Langmuir waves, the mobility of the ions should not affect the saturation induced by either wave breaking or particle trapping. These simulations, as well as the experimental spectrum shown below would suggest that these mechanisms are probably not limiting the amplitude of the Langmuir waves for small values of $k \lambda_{\text {De }}$.

Both particle trapping and wave breaking are much more likely to affect the saturation of Langmuir waves which are driven with large values of $k \lambda_{\text {De }}$. These mechanisms could be responsible for the saturation of Langmuir waves driven with $k \lambda_{\mathrm{De}}>0.4$ with other mechanisms such as the Langmuir decay instability and other secondary decay processes causing saturation for smaller values of $k \lambda_{\text {De }}$. This is in fact consistent with the spectrum shown in figure 2.10.3 which shows the Raman spectrum being cut off for $k \lambda_{\mathrm{De}}<0.6$. This cutoff is usually attributed to Landau damping, however, in many cases stimulated Raman scattering remains above the convective threshold for large values of $k \lambda_{D e}$ due to the decrease in the collisional damping of the scattered electromagnetic wave as the density decreases.

Wave breaking and particle trapping are much more likely in shortpulse high-intensity experiments because the waves can grow on a time scale faster than many of the ion wave saturation mechanisms described above can occur. Therefore, the waves are likely to grow to much higher amplitudes 
than in the long pulse experiments where the growth rate is comparable to the growth rate for many of the ion wave saturation mechanisms. There is

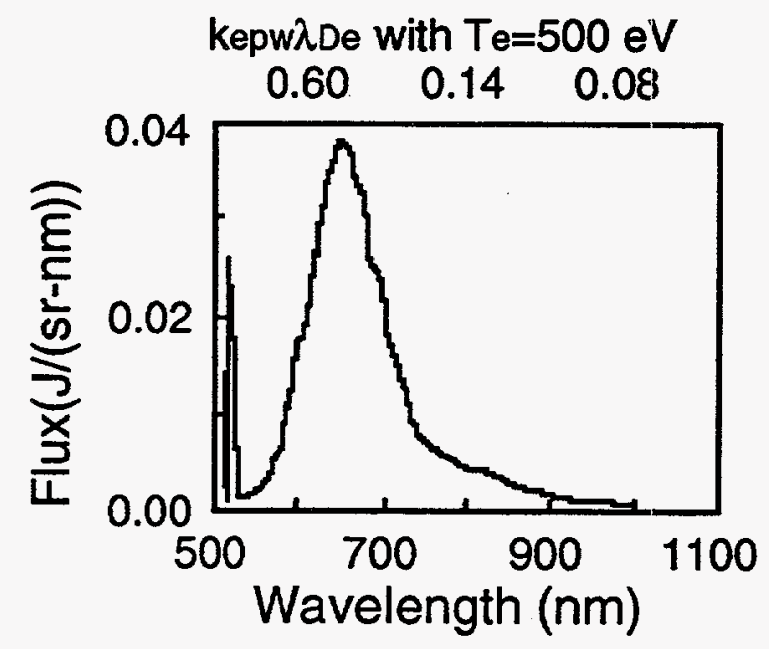

Figure 2.10.3 Flux of stimulated Raman scattering driven from a gold solid target. This graph was reproduced from a paper by Shepard et al.[55]. The bottom horizontal axis represents the wavelength of the scattered light while the top horizontal axis represents the corresponding value of $k \lambda_{D e}$ of the Raman generated Langmuir wave. This figure shows that the Raman flux peaks at an intermediary value of $k \lambda_{\text {De }}$ and then decreases as $k \lambda_{\text {De }}$ decreases.

evidence that these short-pulse lasers can drive significantly larger reflectivity levels of Raman than their long-pulse counterparts with equivalent plasma parameters[37,74]. The increased reflectivity would occur even if ion wave saturation mechanisms were active due to the significant increase in the growth rate for the Raman process resulting from the higher intensity interaction beam. An interesting and rather simple experiment or simulation would be to see if the spectral saturation of Raman scattering in short-pulse 
high-intensity experiments actually increased with decreasing values of $k \lambda_{\text {De }}$ in contrast with long-pulse experiments but in accordance with the assumption that the saturation is due to particle trapping or wave breaking. This experiments would best be performed from a solid target experiment in which all densities are present. The plasma could be preformed with a longpulse laser and then on different shots a high-intensity short-pulse laser and a long-pulse laser could be used to drive stimulated Raman scattering and their resulting spectrum compared. This would then only require measuring the reflectivity at discrete wavelengths of the Raman scattered signal, preferably in a solid target experiment where all densities are present. If ion wave mechanisms are not contributing to the saturation of stimulated Raman scattering, then wave breaking and particle trapping would produce a unique spectrum which would be quite different than many of the ion wave saturation mechanisms described in the previous sections. 


\section{Chapter 3}

\section{Electromagnetic decay instability}

\subsection{Introduction}

This chapter examines the electromagnetic decay instability(EDI) and its role in laser-produced plasmas. The electromagnetic decay instability provides another channel through which parametric instabilities involving Langmuir waves can saturate. As a specific example, the conditions for which EDI is an absolute instability are found for the case where this instability is pumped by the Langmuir wave associated with stimulated Raman scattering. In the case where EDI is pumped by the Langmuir waves associated with two plasmon decay, EDI presents an explanation for $\omega_{\mathrm{o}} / 2$ emission from laser-plasmas which is consistent with experimental observations. In addition, the scattering of Langmuir waves off of ion acoustic waves near the critical surface is shown to provide an enhanced noise source from which stimulated Brillouin scattering can grow. This enhanced noise source can then appear as an apparent Brillouin signal. 
Controlling parametric instabilities has been a major focus of the inertial confinement fusion community for many years[16]. A key step in this process is to understand the saturation mechanisms which limit the amplitude of the waves participating in these instabilities. This chapter addresses one particular saturation mechanism in which a Langmuir wave decays into an electromagnetic wave and an ion acoustic wave. This saturation mechanism can directly or indirectly affect every three-wave parametric instability present in laser-produced plasmas.

When one of the daughter waves driven by a particular instability becomes large enough, it can become the pump for a secondary decay process. As the daughter waves driven by the secondary instability grow, the primary instability may saturate if enough energy is removed to pump the secondary instability. The secondary instability, therefore, introduces an effective "nonlinear" damping into the primary instability[9]. In addition, parametric instabilities may saturate due to the frequency shift resulting from the coupling to secondary instabilities and also due to the self consistent detuning caused by changes to the background density $[17,38]$.

The electromagnetic decay instability(EDI), which is the subject of this chapter, involves the decay of a Langmuir wave into an electromagnetic wave and an ion acoustic wave. Because the ion acoustic wave is a low frequency wave, the frequency of the electromagnetic daughter wave is very close to the frequency of the Langmuir wave pump. This has led to the use of EDI as an explanation for electromagnetic emission near the plasma frequency observed in laboratory electron beam instability experiments[41], type III solar bursts[42,43], and recently in ionospheric heating 
experiments[44]. The simultaneous evolution of EDI with the decay of the Langmuir wave into another Langmuir wave and an ion acoustic wave has also been studied[45]. In the case of type III solar bursts, electrons stream from the sun during solar flares and excite Langmuir waves via the bump-on-tail instability. These Langmuir waves can then undergo a decay into an electromagnetic wave and an ion acoustic wave (EDI). EDI then provides one of several explanations for the radio emission recorded during type III solar bursts[42].

Most of the parametric instabilities present in laser-produced plasmas posses daughter Langmuir waves which can drive EDI. In particular, the Langmuir waves generated in stimulated Raman scattering, two plasmon decay, ion acoustic decay, oscillating two stream instability, and the Langmuir decay instability $[16,46,75]$ may all decay through EDI. In the case of stimulated Brillouin scattering, EDI may still play a role by creating an enhanced noise level from which stimulated Brillouin scattering may grow.

To be resonant, EDI must satisfy frequency and wavenumber matching conditions. The frequency matching condition is given by $\omega_{\mathrm{epw}}=\omega_{\mathrm{s}}+\omega_{\mathrm{ia}}$ and the wavenumber matching condition by $\vec{k}_{\text {epw }}=\vec{k}_{s}+\vec{k}_{i a}$, where $\omega_{\mathrm{epw}}, \overrightarrow{\mathrm{k}}_{\mathrm{epw}} ; \omega_{\mathrm{s}}, \overrightarrow{\mathrm{k}}_{\mathrm{s}} ; \omega_{\mathrm{ia}}, \overrightarrow{\mathrm{k}}_{\mathrm{ia}}$ are the frequency and wavenumber for the Langmuir wave, the electromagnetic wave, and the ion acoustic wave respectively. The frequency matching condition in conjunction with the dispersion relations imply that the wavenumber of the electromagnetic wave is much less than that of the Langmuir wavenumber or, more precisely $\left|\mathbf{k}_{\mathrm{s}}\right| \approx \sqrt{3} \frac{\mathrm{v}_{\text {th }}}{\mathrm{c}}\left|\mathrm{k}_{\mathrm{epw}}\right|$, where $\mathrm{v}_{\text {th }}$ is the electron thermal velocity and $\mathrm{c}$ is the speed of light. 
The homogeneous growth rate for EDI, as with all parametric instabilities,[16] can be calculated by Fourier transforming the fluid equations and Maxwell's equations, neglecting non-resonant terms. This procedure assumes kinetic effects are not dominant. The homogeneous growth rate calculated by this method is[41]

$$
\gamma_{\mathrm{EDI}} \approx \frac{1}{4}\left(\frac{\mathrm{n}_{\mathrm{epw}}}{\mathrm{n}_{\mathrm{oe}}}\right) \frac{\omega_{\mathrm{pi}}}{\left(1+\mathrm{k}_{\mathrm{ia}}^{2} \lambda_{\mathrm{De}}^{2}\right)}\left(\frac{\mathbf{k}_{\mathrm{ia}}^{2}}{\mathrm{k}_{\mathrm{epw}}^{2}}\right) \frac{\omega_{\mathrm{epw}}\left(\hat{\mathrm{e}}_{\mathrm{A}} \cdot \hat{\mathrm{e}}_{\mathrm{epw}}\right)}{\sqrt{\mathrm{k}_{\mathrm{ia}} \mathrm{c}_{\mathrm{s}} \sqrt{\omega_{\mathrm{s}}}}}
$$

where $\omega_{\mathrm{pi}}, \omega_{\mathrm{epw}}, \omega_{\mathrm{s}}$ are the ion plasma frequency, the Langmuir wave frequency and the scattered light wave frequency respectively; $c_{s}$ and $\lambda_{D e}$ are the sound speed and the electron Debye length; $n_{e p w} / n_{o e}$ is the amplitude of the Langmuir wave pump; and $\left(\hat{\mathrm{e}}_{\mathrm{A}} \cdot \hat{\mathrm{e}}_{\mathrm{epw}}\right)$ is the dot product of the electric field vectors for the Langmuir wave and the scattered light wave. The growth rate is maximum when the electric fields of the Langmuir wave and the electromagnetic wave are aligned. This corresponds to direct sidescatter of the electromagnetic wave in a plane perpendicular to the propagation direction of the Langmuir wave.

The following sections discuss some specific cases where EDI can be driven in laser produced plasmas. Section 3.2 discusses the effects of EDI when it is driven by two plasmon decay and compares this to previous experimental observations. Section 3.3 examines the scattering version of EDI at the critical surface and its consequences for stimulated Brillouin scattering, as well as the ion acoustic decay instability. In section 3.4 , the conditions in 
which EDI can be an absolute instability when driven by the Langmuir wave associated with stimulated Raman scattering are found.

\subsection{Electromagnetic decay instability from two plasmon decay}

EDI can be pumped by the Langmuir waves associated with two plasmon decay, a parametric instability involving the decay of an incident electromagnetic wave into two Langmuir waves. Since the frequency of these two Langmuir waves is approximately half the laser frequency, radiation scattered at odd half-harmonics of the laser has been attributed to two plasmon decay. Because EDI involves the decay of a Langmuir wave into an electromagnetic wave and an ion acoustic wave, it provides a potential explanation for the electromagnetic emission near half the laser frequency observed in laser-plasma experiments.

Figure 3.2.1 shows a typical spectrum of light near half the laser harmonic. This spectrum has been reproduced from Seka et al.[76] with permission of the first author Wolf Seka. The spectrum shows two broad peaks $\left(\frac{\Delta \omega}{\omega} \approx 0.02\right)[76,77]$ which are $\operatorname{red}(c)$ and blue(b) shifted from half the laser frequency and one narrow peak $\left(\frac{\Delta \omega}{\omega} \approx 0.004\right)[76,77]$ which is $\operatorname{red}(\mathrm{a})$ shifted by approximately half the amount of the broad red peak(c). This narrow feature(a) has been attributed to the high frequency mixed polarization instability, $[76,77]$ whose theory has been developed by Afeyan[78].

The frequency shifts of the two Langmuir waves associated with two plasmon decay can be calculated from the dispersion relation. When plasma 
flow is included in the calculation and assumed to be antiparallel to the incident pump wavenumber, the plasma waves are up and down shifted from half the laser frequency by[79] $\Delta \omega= \pm\left(\frac{\vec{k}_{\mathrm{epw}} \cdot \overrightarrow{\mathrm{k}}_{\mathrm{o}}}{\mathrm{k}_{\mathrm{o}}}-\frac{1}{2} \mathrm{k}_{\mathrm{o}}\right)\left(\frac{3 \sqrt{3}}{2} \frac{\mathrm{v}_{\mathrm{th}}^{2}}{\mathrm{c}}-\mathrm{u}_{\mathrm{f}}\right)$, where $\overrightarrow{\mathbf{k}}_{\mathrm{epw}}$ is the wavenumber of the blue plasmon, $\overrightarrow{\mathbf{k}}_{\mathbf{o}}$ is the wavenumber of the incident wavenumber, $v_{t h}$ is the thermal electron velocity, and $u_{f}$ is the flow velocity of the plasma.

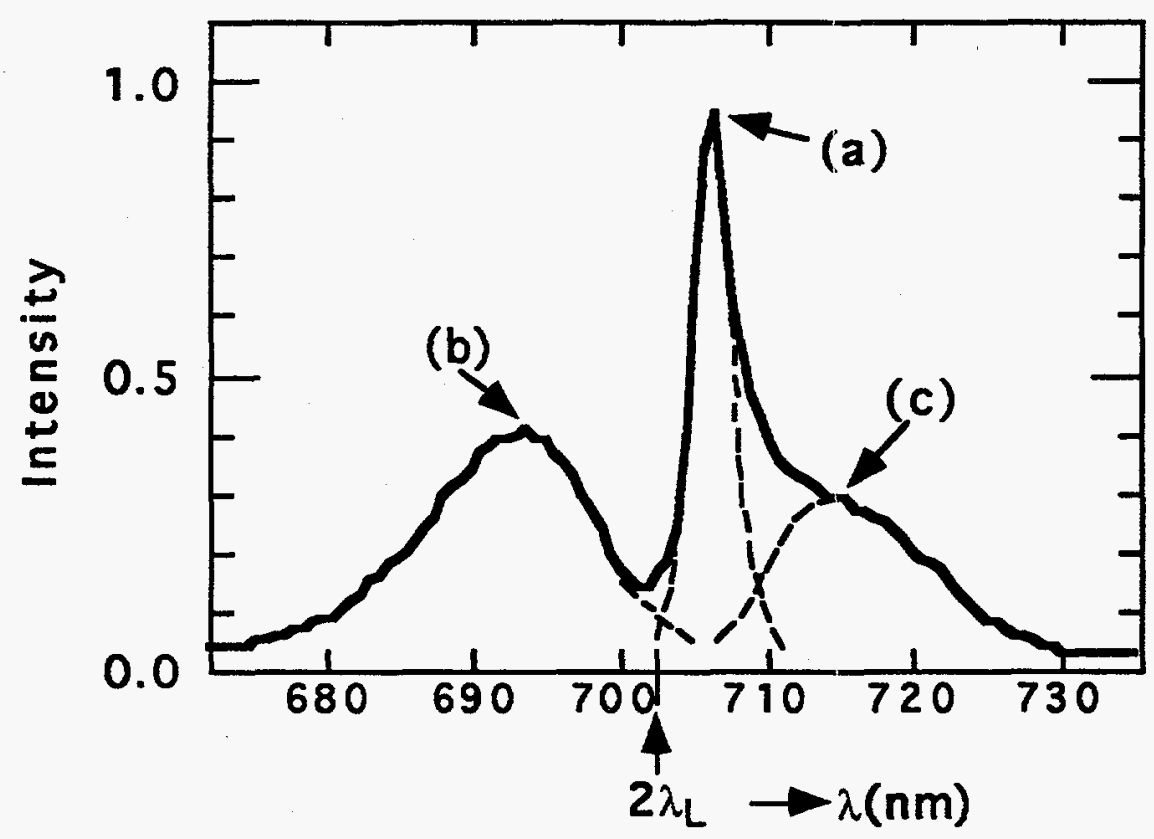

Figure 3.2.1 Plot of the $\omega_{0} / 2$ emission observed from a solid target experiment. The vertical axis shows the relative emission while the horizontal axis shows the wavelength of the scattered light. This graph has been reprinted with permission of Wolf Seka.

Two explanations have appeared in the literature for the broad features shown in figure $3 \cdot 2 \cdot 1[76,77,80]$. The first explanation involves Thomson scattering of the incident laser off of the electron plasma waves associated 
with two plasmon decay. The red shifted peak has been attributed to Thomson scattering of the incident laser from the blue shifted plasmons associated with two plasmon decay, while the blue shifted peak has been attributed to Thomson scattering of reflected light from the critical surface off of the red plasmons associated with two plasmon decay. The second explanation involves the inverse resonance absorption of the Langmuir waves associated with two plasmon decay. In inverse resonance absorption, $[81,82]$ the electric field of a Langmuir wave, at its turning point, tunnels up the density gradient where some fraction of its energy excites electric and magnetic fields at the critical surface. These fields travel down the density gradient and drive an electromagnetic wave at its turning point which then leaves the plasma. Recently a third explanation has been used to describe this electromagnetic emission[59]. This explanation involves the unstimulated coupling of Langmuir waves and ion acoustic waves to drive electromagnetic waves and is the scattering analog of EDI.

The collisional damping on the Langmuir waves limit how far the waves can travel and still retain a significant amount of their energy. Therefore, any explanation for the electromagnetic emission which requires propagation of the Langmuir waves must consider their subsequent damping. The collisional damping of the Langmuir wave's energy as it travels up the density gradient to its turning point can be estimated using the WKB method[16] to be approximately $\exp \left[\frac{-2 \mathbf{k}_{\mathrm{epw}} \lambda_{\mathrm{De}} v_{\mathrm{ei}} \mathrm{L}}{\mathrm{v}_{\mathrm{th}}}\left(1-\frac{\mathbf{k}_{\mathbf{y}}}{\mathbf{k}_{\mathrm{epw}}}\right)\right]$, where $v_{\mathrm{ei}}$ is the electron-ion collision frequency and $k_{y}$ is the Langmuir wavenumber perpendicular to the density gradient. For the experiments discussed in this chapter,[76,77,83,84] a Langmuir wave driven at an angle of 10 degrees to the 
density gradient would retain less than 6 percent of its energy when it reaches its turning point.

There are several problems with the Thomson scattering explanation of $\omega_{0} / 2$ emission from plasmas. Electromagnetic emission near $3 \omega_{0} / 2$ has been measured simultaneously with the $\omega_{0} / 2$ emission and both of these features have been attributed to Thomson scattering of the pump beam off of the Langmuir waves associated with two plasmon decay. The $3 \omega_{0} / 2$ features would correspond to Thomson upscattering, while the $\omega_{\mathrm{o}} / 2$ features would correspond to the Thomson downscattering. As shown in figure 3.2.2, the $\omega_{0} / 2$ feature, which is damped more than the $3 \omega_{0} / 2$ feature, can contain nearly four orders of magnitude more energy than the $3 \omega_{0} / 2$ feature. Figure 3.2.2 has also been reprinted from Seka et al.[76]. Using the models developed in section 3.3 and $4.3[7,80,85]$, the ratio of intensities of the Thomson downscattering process, $I_{1 / 2}$, to the Thomson upscattering process, $I_{3 / 2}$, which is responsible for the $3 \omega_{0} / 2$ features, is the smaller of $\left\{\mathrm{I}_{1 / 2} / \mathrm{I}_{3 / 2}=0.33 \mathrm{k}_{3 / 2} \mathrm{k}_{1 / 2}^{-1}, \mathrm{I}_{1 / 2} / \mathrm{I}_{3 / 2} \approx 28\left(\omega_{0}^{-1} \mathrm{~L}_{\mathrm{n}}^{-1} \mathrm{c}\right)^{0.13}\left(\mathrm{~L}_{\mathrm{n}}^{2} \kappa_{1 / 2}^{\prime}\right)^{0.35}\left(\kappa_{3 / 2}^{\prime} \kappa_{1 / 2}^{\prime-1}\right)\right\}$, which for $k_{e p w} \lambda_{D e}<0.1$ is less than approximately 330 . The relative magnitudes between the $3 \omega_{0} / 2$ features and the $\omega_{0} / 2$ features is then inconsistent with the interpretation that the $\omega_{0} / 2$ feature is the result of Thomson scattering.

One apparent solution to the difference in magnitude of the $3 \omega_{0} / 2$ and $\omega_{0} / 2$ features would be to assume that the $3 \omega_{0} / 2$ feature resulted from Thomson scattering, while the $\omega_{0} / 2$ feature was the result of stimulated Raman scattering. Specifically, if the Langmuir waves associated with two 
plasmon decay became resonant with stimulated Raman scattering, through refraction or scattering, they could be amplified, resulting in a much higher

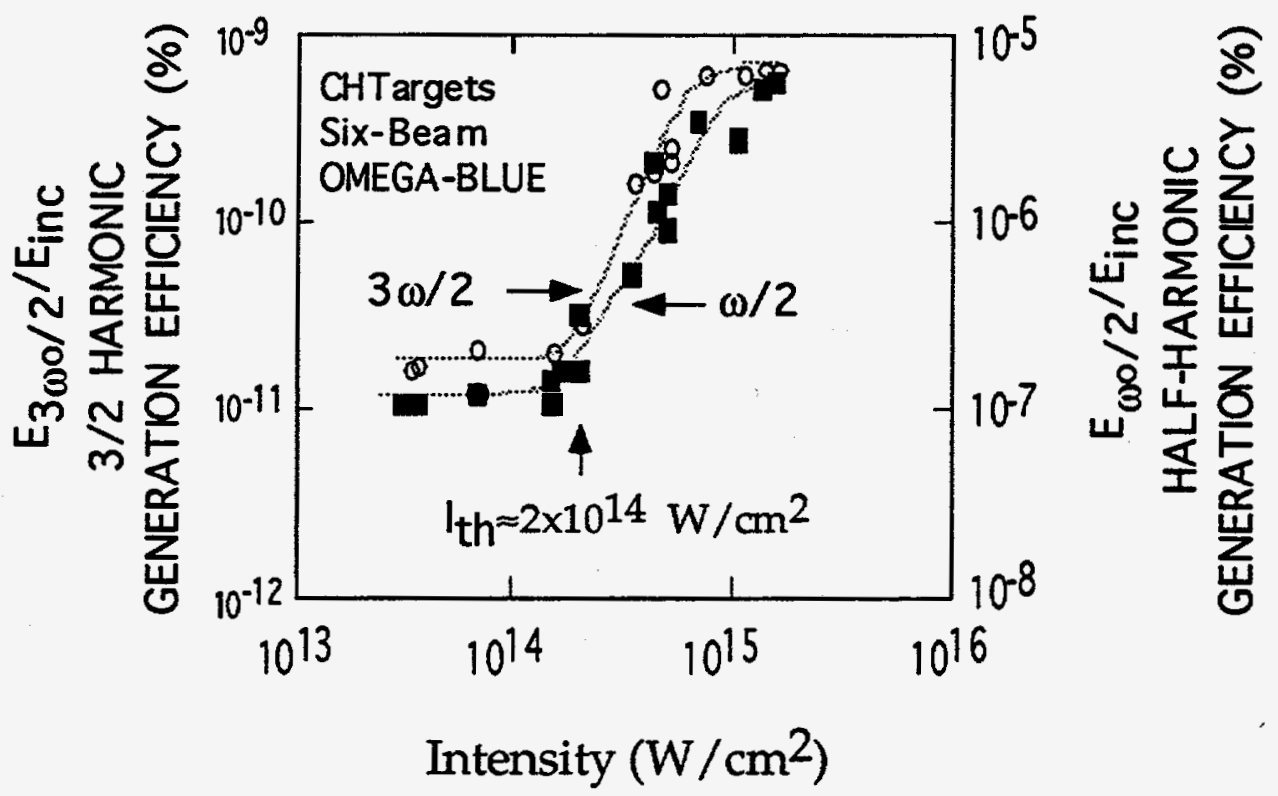

Figure 3.2.2 Plot of the absolute magnitude of $3 \omega_{0} / 2$ emission and $\omega_{0} / 2$ emission as a function of incident laser intensity. The left vertical axis shows the absolute $3 \omega_{0} / 2$ emission and the right vertical axis shows the absolute $\omega_{\mathrm{o}} / 2$ emission while the horizontal axis shows the intensity of the incident laser. This graph has been reprinted with permission of Wolf Seka.

emission of $\omega_{0} / 2$ light than $3 \omega_{0} / 2$ light. One of the problems with this explanation can be seen in the spectrum shown in figure 3.2.1. The $\omega_{0} / 2$ emission consists of two broad peaks which are red(c) and blue(b) shifted from the laser. Although the red peak(c) could be amplified by stimulated Raman scattering, the blue peak(b), as discussed in chapter 2, could not. The resulting spectrum should then only show the red peak(c) due to the limited dynamic range of the streak camera. Figure 3.2.1 shows that the broad blue peak(b) 
contains in fact approximately twice the energy as the broad red peak(c). The similarity in magnitude of the two broad features precludes stimulated Raman scattering as the explanation for the two broad peaks observed in the experiments.

The second explanation of the $\omega_{0} / 2$ feature, which appears in the literature, is the conversion of Langmuir energy into electromagnetic energy by inverse resonance absorption. The conversion efficiency for inverse resonance absorption is expressed in terms of the parameter $q=\left(\frac{\omega_{e p w} L}{c}\right)^{0.67} \sin ^{2} \theta$, where $\omega_{e p w}$ is the Langmuir wave frequency and the electromagnetic wave frequency, $\mathrm{L}$ is the density scalelength, $\mathrm{c}$ is the speed of light, and $\theta$ is the angle between the density gradient and the converted electromagnetic wavenumber at the plasma vacuum boundary[16,81,82]. For the process to be greater than one percent efficient, the parameter q must be less than approximately 2.5 which limits the perpendicular wavenumber to $\mathrm{k}_{\mathrm{y}}=\frac{\mathrm{k}_{\mathrm{o}}}{\sqrt{3}} \sqrt{\mathrm{q}}\left(\frac{\omega_{\mathrm{epw}} \mathrm{L}}{\mathrm{c}}\right)^{-0.33} \leq 0.115 \mathrm{k}_{0}$, where $\mathrm{k}_{0} \approx \sqrt{3} \frac{\omega_{\mathrm{pe}}}{\mathrm{c}}$ is the wavenumber of the incident laser at the quarter critical surface. The perpendicular component of the Langmuir wave can be related to the electromagnetic wave angle through the relation $\mathrm{k}_{\mathrm{y}}=\frac{\omega_{\mathrm{epw}}}{\mathrm{c}} \sin \theta=\frac{\omega_{\mathrm{epw}}}{\mathrm{c}} \sqrt{\mathrm{q}}\left(\frac{\omega_{\mathrm{epw}} \mathrm{L}}{\mathrm{c}}\right)^{-0.33}$. This shows that a Langmuir wave with a perpendicular component of its wavenumber greater than the Langmuir wave frequency divided by the speed of light cannot undergo inverse resonance absorption because the required electromagnetic wave would not be a normal mode of the plasma. 


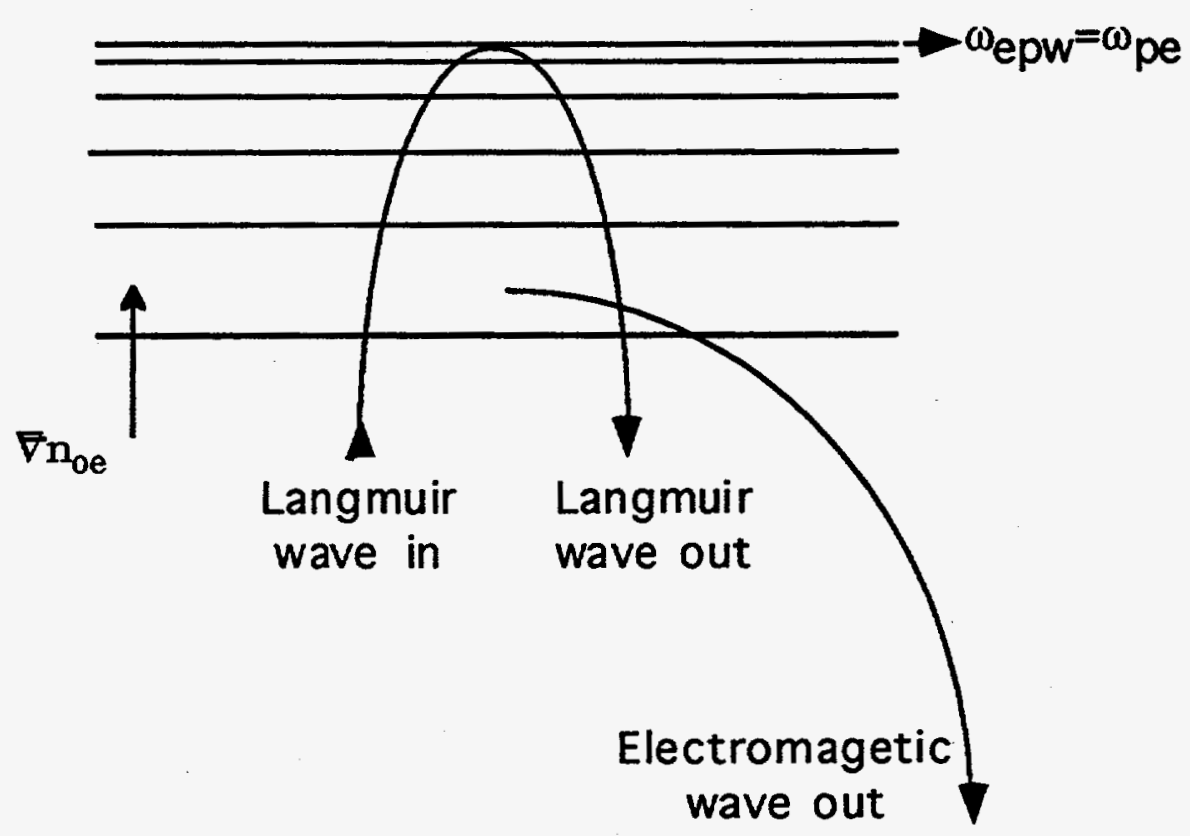

Figure 3.2.3 Schematic representation of inverse resonance absorption in an inhomogeneous plasma. The electric field then tunnels up the density gradient and back, driving a Langmuir wave and an electromagnetic wave which have the same perpendicular component to their wavenumber as the original Langmuir wave.

Because of the restrictions on the perpendicular component of the wavenumber, the phase space of plasmons available for efficient conversion into electromagnetic radiation is very small. Even with considerable scattering of the Langmuir waves, only those plasmons traveling up the density gradient with the specified perpendicular component of their wavenumber would undergo appreciable conversion into electromagnetic waves. Likewise, most of their energy would be lost before they reached their critical surface. Without significant perturbations to the background density, 
inverse resonance absorption does not present itself as a likely candidate to explain the emission observed in experiments[76,77].

There are several ways in which the background density could be perturbed to increase the amount of inverse resonance absorption. If two plasmon decay occurs in laser hotspots, then the hotspots could provide a broader angular range for the density gradient $[76,86]$. The ponderomotive force of the laser hotspot would cause a density cavity in which the two plasmon decay Langmuir waves could become trapped. These waves could then undergo inverse resonance absorption on the walls of the cavity. Assuming a simple pressure balance in a homogeneous plasma, the hotspot intensity divided by the average intensity, $\alpha$, required to trap a Langmuir wave is

$$
\alpha=1+\frac{\left(3 k_{\mathrm{epw}}^{2} \lambda_{\mathrm{De}}^{2}\right)\left(k \mathrm{~T}_{\mathrm{e}} \mathrm{n}_{\mathrm{oe}}\right)}{\mathrm{I}_{\mathrm{av}}}\left(\frac{\sqrt{3} \mathrm{c}}{2}\right),
$$

where $k T_{e} n_{o e}$ is the plasma pressure and $I_{a v}$ is the average pump intensity. $\alpha$ would have a value of 4 assuming $\mathrm{k}_{\mathrm{epw}} \lambda_{\mathrm{De}}=0.2, \mathrm{I}_{\mathrm{av}}=8 \times 10^{14} \mathrm{~W} / \mathrm{cm}^{2}, \mathrm{~T}_{\mathrm{e}}=1$ $\mathrm{keV}$, and $\mathrm{n}_{\mathrm{oe}}=2.25 \times 10^{21} \mathrm{~cm}^{-3}$. The reduced local scalelength inside the cavity increases the range of angles for efficient resonance absorption.

Inverse resonance absorption can also occur due to changes in the background density caused by ion acoustic waves. Large amplitude ion acoustic waves can cause the local electron density to become overdense to the Langmuir waves. These Langmuir waves can then undergo inverse resonance absorption on the ion acoustic wave itself. This eliminates the need for the Langmuir waves to travel large distances, and the need for the 
red plasmons to undergo decay or be scattered up the density gradient. The amplitude of the ion acoustic waves, $n_{i a} / n_{o i}$, must be greater than $3\left(k_{\text {epw }} \lambda_{\mathrm{De}}\right)^{2}$. Therefore, it is less likely that this process can be used to convert Langmuir waves with large $k_{e p w} \lambda_{D e}$, however, ion acoustic waves with amplitudes of $25 \%$ have been reported near the quarter critical surface in $\mathrm{CO}_{2}$ experiments[17]. This process could even take place in an overall homogeneous plasma, relying only on the local density gradients resulting from the ion fluctuations. In this process, the "local" scalelength is replaced by the wavelength of the ion acoustic fluctuation which leads to a much larger angular range since q, which must be less than 2.5 for efficient conversion, is proportional to $\mathrm{L}^{2 / 3}$. In the case of conversion on ion acoustic waves, much of the electric field will tunnel through the ion wave converting a small percentage of the Langmuir wave energy into electromagnetic waves on either side of the density fluctuation.

Both hotspots and large level ion waves provide a means whereby a larger spectrum of Langmuir waves can undergo inverse resonance absorption. The resulting electromagnetic waves will be approximately symmetrically displaced about half the frequency of the incident laser. The spectrum shown in figure 3.2.1, however, shows a slight red shift of the spectrum about half the laser frequency which is not predicted by inverse resonance absorption.

It has been shown that electromagnetic emission near $\omega_{0} / 2$ can result from inverse plasmon-phonon decay which is the coupling of Langmuir waves and ion acoustic waves which then radiate electromagnetic waves[59]. However, this scattering process requires the ion wave amplitudes to be 
much larger than thermal levels to explain the signals observed. These experiments indicated that nearly twenty five percent of the energy contained in the hot electrons attributed to the Langmuir waves was converted into electromagnetic emission near $\omega_{0} / 2$. The ion acoustic wavenumbers must also be approximately the same magnitude and direction as the Langmuir wave it is scattering from. Therefore, relying on this scattering process requires that the appropriate ion waves be part of the ion acoustic spectra generated by the coupling, decay, or collapse of the two plasmon decay and stimulated Raman scattering Langmuir waves[59]. EDI can lead to much stronger emission and greatly enhanced ion waves when compared to scattering.

As a result of EDI, the Langmuir waves generated by two plasmon decay can produce electromagnetic emission near $\omega_{\mathrm{O}} / 2$. In the subsequent decay of these Langmuir waves into electromagnetic waves and ion acoustic waves, the scattered light frequencies of the secondary daughter waves, assuming the contribution from inhomogeneity is ignorable, would be

$$
\begin{aligned}
& \omega_{\text {sblue }}=\frac{\omega_{0}}{2}+\left(\frac{\vec{k}_{\text {epw }} \cdot \vec{k}_{0}}{\mathbf{k}_{0}}-\frac{1}{2} \mathbf{k}_{0}\right)\left(\frac{3 \sqrt{3}}{2} \frac{v_{\text {th }}^{2}}{c}\right)-c_{s} k_{\text {epw } 1}+\frac{u_{\mathrm{f}} \mathbf{k}_{0}}{2} \text { and } \\
& \omega_{\text {sred }}=\frac{\omega_{0}}{2}-\left(\frac{\vec{k}_{\text {epw }} \cdot \vec{k}_{0}}{\mathbf{k}_{0}}-\frac{1}{2} \mathbf{k}_{0}\right)\left(\frac{3 \sqrt{3}}{2} \frac{v_{\text {th }}^{2}}{c}\right)-c_{\mathrm{s}} k_{\text {epw } 2}+\frac{u_{\mathrm{f}} \mathbf{k}_{0}}{2}
\end{aligned}
$$

where $\omega_{\text {sblue }}$ is the scattered light frequency resulting from the blue plasmon and $\omega_{\text {sred }}$ is the scattered light frequency produced by the red plasmon. Equations 3.2.2 and 3.2.3 show that EDI does allow for an overall red shift of 
the $\omega_{0} / 2$ spectrum. The maximum energy transferred to electromagnetic emission is limited by the total energy contained in the Langmuir waves associated with two plasmon decay. Previous experiments have inferred from the measured $x$-ray emission that approximately $10^{-4}$ of the laser energy is contained in hot electrons attributed to two plasmon decay[83,84]. These experiments found a close correlation between the relative levels of hot electrons and scattered $3 \omega_{0} / 2$ emission observed allowing them to conclude that the electrons resulted from two plasmon decay. In similar experiments $5 \times 10^{-6}$ of the laser energy was found in $\omega_{0} / 2$ emission[76]. When the damping of the $\omega_{0} / 2$ emission is accounted for, the energy contained in the $\omega_{0} / 2$ light represents approximately $25 \%$ of the energy observed in the hot electrons. This illustrates that there is enough energy contained in the Langmuir waves themselves to explain the level of $\omega_{0} / 2$ emission seen in experiments. It is also interesting to note that stimulated Brillouin scattering, which shares the same daughter waves as EDI, typically has reflectivity levels close to that inferred from the experiments described above. As an instability, EDI amplifies both the ion acoustic waves and the electromagnetic waves from which it grows. Therefore, unlike inverse plasmon-phonon decay, EDI does not require other processes to enhance and broaden the ion acoustic wave spectrum.

\subsection{Seeding of stimulated Brillouin scattering and saturation of the ion acoustic decay instability}


Stimulated Brillouin scattering, which is an instability in which an electromagnetic wave drives a scattered electromagnetic wave and an ion acoustic wave, can be seeded by nonthermal electromagnetic waves generated near the critical surface. Near the critical surface of the plasma, the incident laser can excite the ion acoustic decay instability. The ion acoustic decay instability, also known as plasmon-phonon decay, is a parametric instability in which an incident electromagnetic wave decays into a Langmuir wave and an ion acoustic wave. This coupling process is illustrated in figure 3.3.1. The Langmuir wave and ion acoustic wave spectra resulting from the ion acoustic decay instability can then couple together generating a transverse current. This current drives an electromagnetic wave close to the frequency of the laser (which initially excited the ion acoustic decay instability). This coupling process generates electromagnetic waves which can be many orders of magnitude above thermal level. These electromagnetic waves, wherever they are locally resonant, provide an enhanced noise source from which stimulated Brillouin scattering can grow. This can be much larger than the electromagnetic noise source from which stimulated Brillouin scattering grows, which is set by Thomson scattering of the pump from thermal ion waves. This process can also give the appearance of an enhanced level of stimulated Brillouin scattering.

A model can be constructed which shows the electromagnetic wave amplitude, near its turning point, produced by the scattering of a Langmuir wave from an ion acoustic wave[7,85]. The electromagnetic wave is driven 


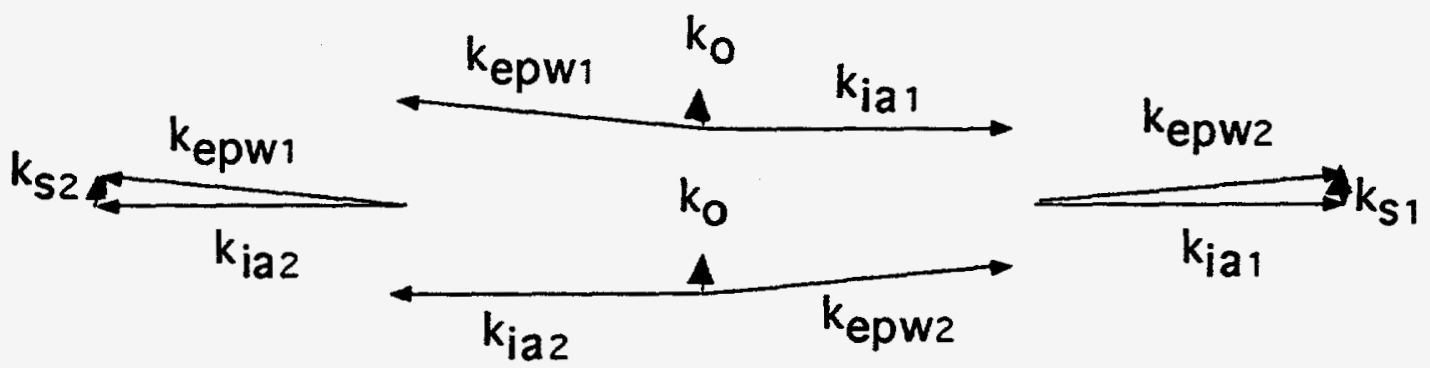

Figure 3.3.1 Wavevector diagram showing the coupling process which leads to electromagnetic emission near the plasma frequency. The two wavevector diagrams in the center represent two possible decay triangles belonging to the ion acoustic decay instability. The ion acoustic wave from one of these decay triangles can couple to the Langmuir wave of the second decay triangle and drive an electromagnetic wave close to the Langmuir wave frequency.

due to the transverse current formed by the coupling of a Langmuir wave and an ion acoustic wave which is given by

$$
\left(\frac{\partial^{2}}{\partial \mathrm{t}^{2}}+\omega_{\mathrm{pe}}^{2}\left(1+\frac{\mathrm{x}}{\mathrm{L}_{\mathrm{n}}}\right)-c^{2} \frac{\partial^{2}}{\partial \mathrm{x}^{2}}\right) \frac{\mathrm{qA}}{\mathrm{m}_{\mathrm{ec}} \mathrm{c}^{2}}=-\omega_{\mathrm{pe}}^{2} \frac{\mathrm{n}_{\mathrm{ia}}}{\mathrm{n}_{\mathrm{oe}}}\left(\frac{\omega_{\mathrm{epw}}}{c \mathrm{k}_{\mathrm{epw}}} \frac{\mathrm{n}_{\mathrm{epw}}}{\mathrm{n}_{\mathrm{oe}}}\right)
$$

where nepw/noe and $n_{i a} / n_{o e}$ are the pump Langmuir wave amplitude and the ion acoustic amplitude, respectively and $A_{S C}$ is the vector potential of the scattered light wave. The pump Langmuir wave and ion acoustic wave amplitudes are assumed to be of the form $\frac{\mathrm{n}_{\alpha}}{\mathrm{n}_{\mathrm{oe}}}=\frac{1}{2} \Psi_{\alpha}(\mathrm{x}) \exp \left[\mathrm{i}\left(\int \overrightarrow{\mathrm{k}}_{\alpha} \cdot \mathrm{d} \overrightarrow{\mathrm{x}}-\omega_{\alpha} \mathrm{t}\right)\right]+$ c.c.. The scattered vector potential is assumed to be of the form $\frac{\mathrm{qA}_{\mathrm{sc}}}{\mathrm{m}_{\mathrm{e}} \mathrm{c}^{2}}=\frac{1}{2} \Psi_{s}(\mathrm{x}) \exp \left[-i \omega_{\mathrm{s}} t\right]+c . c$. . The steady state form of equation 3.3.1 can be written as 


$$
\left(\frac{\mathrm{d}^{2}}{\mathrm{dx^{2 }}}-\frac{\omega_{\mathrm{pe}}^{2} \mathrm{x}}{\mathrm{c}^{2} \mathrm{~L}_{\mathrm{n}}}\right) \Psi_{s}(\mathrm{x})=\frac{\omega_{\mathrm{pe}}^{2} \omega_{\mathrm{epw}}}{2 \mathrm{c}^{3} \mathrm{k}_{\mathrm{epw}}}\left(\Psi_{\mathrm{epw}} \Psi_{\mathrm{ia}}\right) \exp \left[\frac{\mathrm{i} \mathrm{k}^{\prime} \mathrm{x}^{2}}{2}\right]
$$

where the wavenumber mismatch of the pump Langmuir wave and the ion acoustic wave has been Taylor expanded, $\left(\vec{k}_{i a}(x)+\vec{k}_{\text {epw }}(x)\right) \cdot d \vec{x} \approx K^{\prime} x$, about the perfect phase matching point. Equation 3.3.2 was solved numerically as shown in Appendix 3.1. The resultant scattered vector potential, at the perfect phase matching point, is

$$
\Psi_{\mathrm{s}}(\mathrm{x})=3.3\left[\frac{\omega_{\mathrm{pe}}^{2} \omega_{\mathrm{epw}}}{2 \mathrm{c}^{3} \mathrm{k}_{\mathrm{epw}} \kappa_{\mathrm{edi}}}\left(\Psi_{\mathrm{epw}} \Psi_{\mathrm{ia}}\right)\right]\left(\frac{\omega_{\mathrm{pe}}^{2}}{c^{2} \mathrm{~L}_{\mathrm{n}} \kappa_{\mathrm{edi}}^{1.5}}\right)^{-0.45} .
$$

The scattered vector potential, at the plasma-vacuum interface, is approximately $0.85\left(\omega_{\mathrm{pe}} \mathrm{L}_{\mathrm{n}} \mathrm{c}^{-1}\right)^{-1 / 6}$ times the scattered vector potential, at the turning point shown in equation 3.3.3.

The scattered vector potential resulting from Thomson scattering of the pump beam from thermal ion acoustic waves can be calculated as shown in section 5.3, assuming a linear phase mismatch. This calculation yields a scattered vector potential, at the turning point, of

$$
\left|\Psi_{\text {sc }}\right|_{\text {Thomson }}=\frac{\sqrt{2 \pi}}{\sqrt{\kappa^{\prime} \text { Thomson }} 4} \frac{\omega_{\mathrm{pe}}^{2}}{\mathrm{c}^{2} \mathrm{k}_{\mathrm{sc}}}\left|\Psi_{\mathrm{o}}\right|\left|\Psi_{\text {ia }}\right|_{\text {thernal }}
$$

The ratio of the intensities is then equal to 


$$
\begin{aligned}
& \frac{I_{s c}}{I_{s c_{\text {Thomson }}}}=\left(\frac{c}{v_{g_{\text {Thomson }}}}\right)\left|\frac{A_{s c(\text { in vacuum) }}}{A_{s c_{\text {Thomson }}}}\right|^{2} \approx \\
& 5\left(\frac{\omega_{o}}{\omega_{p e}}\right)^{4}\left(\frac{c}{\omega_{o} L_{n}}\right)^{2.13}\left(L_{n}^{2} \kappa^{\prime}{ }_{\text {edi }}\right)^{1.35}\left|\frac{n_{e p w}}{n_{o e}}\right|^{2}\left(\frac{c}{v_{o s}}\right)^{2} e^{G_{\text {IADI }}} \\
& \frac{\boldsymbol{\kappa}_{\text {Thomson }}^{\prime}}{\kappa_{\text {edi }}^{\prime 2}} \frac{\omega_{\mathrm{o}}^{3} \mathrm{k}_{\mathrm{sc}}}{\mathrm{c}^{3} \mathrm{k}_{\mathrm{epw}}^{2}} \text {, }
\end{aligned}
$$

where $e^{G}$ IADI is the energy gain experienced by the ion acoustic waves participating in the ion acoustic decay instability. The scattered vector potential produced by the coupling of Langmuir waves and ion acoustic waves driven by the ion acoustic decay instability can be several orders of magnitude greater than the vector potential produced by Thomson scattering of the pump off of thermal level ion acoustic waves.

\subsection{Electromagnetic decay instability from stimulated Raman scattering}

There are several circumstances where EDI can be an absolute instability. When parametric instabilities are driven by a spatially localized pump, they can be driven absolute even with a linear wavevector mismatch[28,87]. Therefore, EDI is expected to be an absolute instability when its pump Langmuir wave is sufficiently localized in space. In the case of stimulated Raman backscattering driven near the quarter critical surface or on top of a parabolic profile, the daughter waves will be spatially localized[78]. In 
particular, when stimulated Raman backscattering is driven at the apex of a parabolic profile, the Langmuir wave is localized over a region

$$
1_{\gamma} \approx\left(\frac{\mathrm{v}_{\mathrm{th}}}{\mathrm{c}}\right)^{\frac{1}{3}}\left(\frac{\mathrm{L}_{2}}{\lambda_{\mathrm{o}}}\right)^{\frac{2}{3}}\left(\frac{\omega_{\mathrm{pe}}}{\omega_{0}}\right)^{\frac{-2}{3}}\left(\frac{\mathrm{c}}{\omega_{\mathrm{o}}}\right) \mathrm{f}\left(\frac{\omega_{\mathrm{pe}}}{\omega_{0}}\right)
$$

where $L_{2}$ is the plasma scalelength $\left(n_{o e} \propto \hat{n}\left(1-x^{2} / L_{2}^{2}\right)\right), \lambda_{0}$ is the vacuum wavelength of the pump, and $f\left(\frac{\omega_{\mathrm{pe}}}{\omega_{\mathrm{o}}}\right)=\left[\sqrt{1-2 \frac{\omega_{\mathrm{pe}}}{\omega_{\mathrm{o}}}}\left(\sqrt{1-\left(\frac{\omega_{\mathrm{pe}}}{\omega_{0}}\right)^{2}}+\sqrt{1-2 \frac{\omega_{\mathrm{pe}}}{\omega_{\mathrm{o}}}}\right)\right]^{\frac{1}{6}} \sim 1$. The distance over which the Raman daughter waves are localized is then independent of the Raman growth rate provided the instability is not close to the quarter critical surface where equation 3.4.1 is no longer valid. For EDI to be absolute due to a localized pump, its growth rate must be sufficiently large to satisfy the condition $\frac{\sqrt{3}}{4} \kappa^{\prime} l_{c}^{2}<\kappa^{\prime} l_{\gamma} l_{c}<\sqrt{8 e},[28,87]$ where $\kappa^{\prime}=\frac{d}{d x}\left(k_{\text {epw }}-k_{s}-k_{\text {ion }}\right)$, $l_{c}=\sqrt{V_{s} V_{i o n}} / \gamma_{0}$, and $l_{\gamma}$ is the localization distance given in equation 3.4.1. For $\mathrm{L}_{2}=700 \lambda_{0}, \mathrm{~T}_{\mathrm{e}}=1 \mathrm{keV}, \omega_{\mathrm{pe}} / \omega_{\mathrm{o}}=0.3, \mathrm{kepw}_{\mathrm{De}}=0.2$, the Langmuir wave amplitude must be greater than approximately $2 \%$ for absolute instability to ensue, neglecting damping. This is illustrated below in figure 3.4.1. The program describe in section 2.3 and Appendix 2.4 was used to look at the absolute instability condition shown above. In these figures, the daughter waves were given the same magnitude but opposite group velocities. The Rosenbluth gain was set to $\gamma_{0}^{2} /\left(\kappa^{\prime} V_{1} V_{2}\right)=1.0$ in figures 3.4.1 a-d. Figure 3.4.1 a represents the case where the pump is uniform over space, $l_{\gamma} / l_{C}=\infty$. The 
daughter wave amplitude is shown to saturate at an amplitude of approximately 9.0 with the wave convecting out at the group velocity. Figure $3.4 .1 \mathrm{~b}$ represents the case in which the pump is localized but not yet meeting the condition for absolute instability with $1_{\gamma} / 1_{c}=6.67$. In this case the amplitude saturates at a value of approximately 11.0 with the daughter wave traveling to the right at the group velocity. In figure $3.4 .1 \mathrm{c}$, the condition for
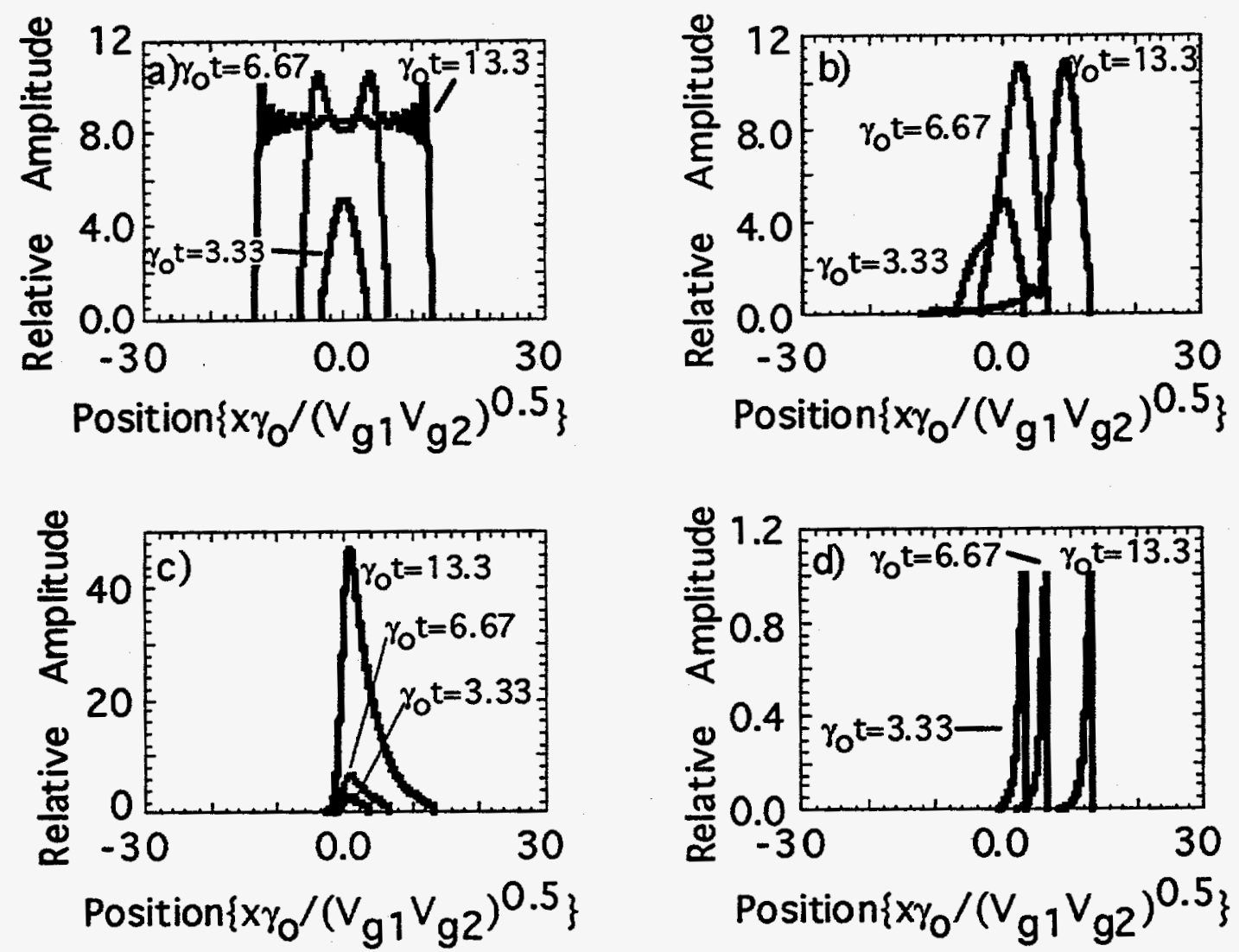

Figure 3.4.1 Finite spatial pump effects on the absolute and convective nature of parametric instabilities. The vertical axes in all graphs represent the relative amplitude while the horizontal axis represents position. Each graph shows the daughter wave amplitude at three different times with the parameter $\gamma_{0}{ }^{2} /\left(\kappa^{\prime} V_{1} V_{2}\right)=1.0$ in figures 3.4.1 a-d. Figure 3.4.1 a represents the 
case where the pump is uniform over space, $l_{\gamma} / l_{\mathfrak{c}}=\infty$. Figure $3.4 .1 \mathrm{~b}$ represents the case in which the pump is localized but not yet meeting the condition for absolute instability with $1_{\gamma} / 1_{\mathrm{c}}=6.67$. In figure $3.4 .1 \mathrm{c}$, the condition for absolute instability is met with $l_{\gamma} / l_{\mathcal{C}}=0.67$. In figure 3.4.1 d, the pump is too localized for absolute instability to ensue with $l_{\gamma} / l_{C}=0.40$.

absolute instability is met with $l_{\gamma} / l_{c}=0.67$. The daughter wave does not saturate due to plasma inhomogeneities and continues to grow in time at a growth rate which is approximately 0.3 times the homogeneous growth rate. In figure 3.4.1 $\mathrm{d}$, the pump is too localized for absolute instability to ensue with $l_{\gamma} / l_{C}=0.40$.

EDI can also become absolute when its daughter electromagnetic wave travels in a perpendicular direction to the density gradient. Figure 3.4.2 illustrates this geometry in the case of stimulated Raman backscattering, where the pump wave for the secondary process is a Langmuir wave traveling along the density gradient. The daughter waves are then an electromagnetic wave perpendicular to the density gradient and an ion acoustic wave which is nearly aligned with the density gradient. EDI is only driven over the distance, perpendicular to the density gradient, in which the Langmuir pump wave is coherent, $\mathrm{L}_{\mathrm{coh}}$. EDI grows for the transit time, $t_{c o h}=L$ coh $/ v g s$, of the electromagnetic wave across this coherence length. Therefore, the maximum energy amplification of the daughter electromagnetic wave is $e^{2 \gamma_{0} t_{c o h}}$, where $\gamma_{0}$ is the homogeneous growth rate given in equation 3.1.1. Assuming a $1 \mathrm{keV}$ plasma with a $0.351 \mu \mathrm{m}$ pump, a Langmuir wave with an amplitude of $n_{e p w} / n_{0 e}=0.05$ driven at ten percent of 
the critical density would require a perpendicular coherence length of nearly $60 \mu \mathrm{m}$ to amplify the daughter electromagnetic wave by $\mathrm{e}^{2 \pi}$.

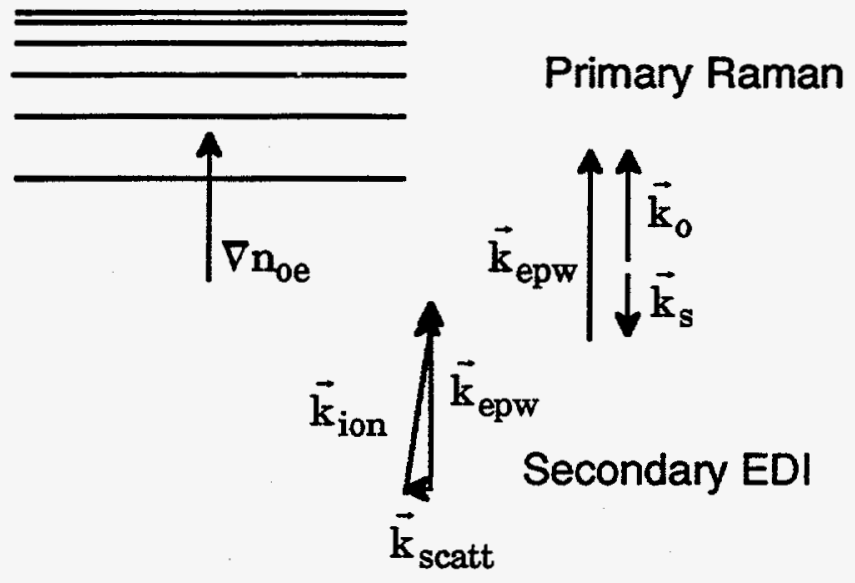

Figure 3.4.2 Schematic representation of absolute electromagnetic decay driven by stimulated Raman scattering. Assuming the laser is incident along the density gradient, stimulated Raman backscatter produces Langmuir waves which also travel in the direction of the density gradient. These Langmuir waves can then decay into a scattered light wave traveling perpendicular to the density gradient causing the instability to be absolute in the direction of the density gradient.

Instabilities can also grow absolutely due to reflections coupling energy from the daughter waves back into the interaction region[29]. This is easily achievable by the same mechanism discussed in section 3.2 regarding hotspots. For hotspot intensities slightly above the average intensity of the laser( $\left(I_{h o t} / I_{a v}<4\right)$, the electromagnetic waves formed by the electromagnetic decay instability can become trapped in the density cavern formed by the hotspot. This scenario, therefore, allows the scattered electromagnetic wave to 
couple back to the interaction region driving the instability absolute. This process also does not require the electromagnetic decay instability to be above the homogeneous threshold for absolute instability.

\subsection{Summary}

The most compelling evidence that the electromagnetic decay instability(EDI) occurs in laser-produced plasmas comes from $\omega_{0} / 2$ emission observed in experiments. This emission has been shown above to be inconsistent with previous interpretations involving Thomson scattering. The observed ratio of $\omega_{0} / 2$ emission to $3 \omega_{0} / 2$ emission implies that Thomson scattering is not responsible for the measured value of $\omega_{0} / 2$ emission. Also it was shown that the broad red shifted feature could not be explained as a resonant scattering process of the incident laser from the blue plasmons even with refraction. Because the red and blue peaks are within a factor of two in energy, it is unlikely that Thomson scattering could explain the observed features. It was also shown that the phase space of blue plasmons capable of undergoing greater than one percent inverse resonance absorption was very small. Additionally these waves would lose a significant amount of their energy due to collisional damping as they traveled to their turning point. Inverse resonance absorption, therefore, requires hotspots or large level ion waves to convert a larger phase space of Langmuir waves into electromagnetic emission. The observed $\omega_{0} / 2$ spectra shows a slight red shift which is not predicted by inverse resonance absorption. Nonetheless, experiments measuring hot electrons have shown that enough energy is 
contained in the two plasmon decay daughter waves to account for the $\omega_{0} / 2$ emission. By using EDI to explain $\omega_{\mathrm{o}} / 2$ emission, the relative levels of $3 \omega_{\mathrm{o}} / 2$ emission and $\omega_{0} / 2$ emission and the amount of energy found in the $\omega_{0} / 2$ feature can be accounted for. EDI also allows for a slight red shifting of the spectrum which can be seen in figure 3.2.1.

Near the critical surface, Langmuir waves and ion acoustic waves can generate transverse currents which drive electromagnetic waves close to the frequency of the incident laser. A model was constructed which shows the ratio of the vector potential resulting from the coupling between Langmuir and ion acoustic waves driven by the ion acoustic decay instability relative to the vector potential driven by Thomson scattering of the incident pump from thermal level ion acoustic waves. These electromagnetic waves can be several orders of magnitude higher than Thomson scattering from thermal levels. They can, therefore, provide an enhanced noise source from which stimulated Brillouin scattering can grow.

The conditions under which EDI could be driven absolute were investigated. EDI can be driven absolute when its daughter electromagnetic wave is driven perpendicular to the density gradient. EDI can also be driven absolute when the pump Langmuir wave is sufficiently localized. For the case of a Raman generated Langmuir wave, these conditions were obtained from previous calculations of the Langmuir wave localization distance[78] and the general condition for absolute instability given a localized pump[28,87]. EDI can also be driven absolute due to the coupling of the daughter wave energy back into the interaction region. If the Raman process is being driven primarily by hotspots in the laser, then for very small hotspot intensities, the 
daughter electromagnetic wave can be trapped in the resultant density cavern and consequently couple back into the interaction region driving the instability absolute.

\subsection{Future Work}

I proposed an experiment to Los Alamos to study Langmuir wave saturation by observing the electromagnetic emission from Langmuir waves. I delayed working on this experiment, however, in the interest of finishing my dissertation. In the proposed experiment, stimulated Raman scattering(SRS) was to be used to generate the Langmuir plasma waves to be studied. In Stimulated Raman Scattering an incident electromagnetic wave decays into an electron plasma wave and a scattered electromagnetic wave. Stimulated Raman Scattering can occur below the quarter critical surface defined by the pump electromagnetic wave. Because this electromagnetic radiation is near the plasma frequency, SRS must be driven with $350 \mathrm{~nm}$ light so that the scattered light can be detected with an S-1 streak camera. The electromagnetic emission from SRS produced Langmuir waves would range from $700 \mathrm{~nm}$ to approximately $1600 \mathrm{~nm}$. With an S-1 streak camera, the range from $700 \mathrm{~nm}$ to approximately $1100 \mathrm{~nm}$, corresponding to stimulated Raman emission from the quarter critical surface down to the tenth critical surface, would be detectable.

In the experiment, I proposed to vary the transverse scalelength to observe the effect this had on the level of stimulated Raman backscattering which has not yet been reported in the literature. According to linear theory, 
the transverse scalelength should not affect stimulated Raman backscatter, assuming the backscatter is along the primary density gradient, however, it could greatly affect secondary instabilities such as the electromagnetic decay instability thereby changing the reflectivity of stimulated Raman backscatter. Likewise, different random phase plates on the interaction beam would affect the hotspot statistics which should effect the electromagnetic decay instability if it is being driven absolute from coupling of the daughter electromagnetic wave back into the interaction region due to trapping caused by the ponderomotive force associated with the hotspot. 


\section{Chapter 4}

\section{Thomson Scattering}

\subsection{Introduction}

Thomson scattering is the primary diagnostic used in the experiments described in the following chapters. This chapter, therefore, presents a review of the theory of Thomson scattering and later discusses the plasma parameters which can, in principle, be obtained through the use of this diagnostic. However, there are limitations due to plasma inhomogeneities which will be discussed in section 4.2. The information given in this chapter is drawn from a number of reviews on Thomson scattering[88-93]. Some of the longer derivations are placed in Appendices. In the following discussion, it will be assumed that there are no zero-order external magnetic fields.

Thomson scattering from plasmas involves the scattering of incident photons by electrons and ions present in the plasma. An incident electromagnetic wave accelerates electrons and ions in the plasma which in 
turn emit radiation that is Doppler shifted due to the thermal velocities of the electrons and ions participating in the scattering process. Because the mass to charge ratio of the electrons is much less than the ions present in the plasma, their acceleration is greater than that of the ions by the ratio $M_{i} / Z_{e}(\geq 1837)$. Consequently the amplitude of the scattered electromagnetic wave resulting from the electrons is greater than the radiation scattered by the ions by this same ratio, $M_{i} / Z_{e}$. The radiation emitted by the plasma particles is incoherent when they are uncorrelated, however, when the electrons participate in collective oscillations in the plasma, they are correlated and the radiation emitted from the electrons participating in the collective oscillation adds coherently. Therefore, the scattered spectrum contains an incoherent component, as well as coherent peaks representing the electrostatic normal modes present in the plasma. These electrostatic normal modes are the Langmuir waves and ion acoustic waves present in the plasma. The ions do play an important role in determining the shape of the scattered spectrum due to the plasma's quasi-neutrality. The electrons shield the ions and hence the coherent scattering observed from ion waves comes from the electrons which are helping to shield the ions. When the wavelength of the ion wave becomes less than the shielding distance, the electron Debye radius, the electrons cannot completely shield the ion charge and hence the Thomson scattering signal from these waves is reduced. This effect would be most apparent in the stimulated Brillouin scattering where the exponential gain is reduced due to the incomplete shielding of the electrons.

Thomson scattering was used in the experiments performed for this dissertation primarily to observe the Langmuir wave spectrum driven by 
parametric instabilities. The next section discusses the calculation of the expected Thomson scattering spectra from thermal level waves in a homogeneous plasma and presents calculated spectra from two of the ionspecies plasmas used in these experiments. This section also reviews the characteristics of the Thomson scattered spectrum which are used to determine various plasma parameters and discusses the ambiguities which inhomogeneities introduce in the interpretation of the Thomson scattered spectra. The final section addresses the issue of Thomson scattering from electrostatic waves in inhomogeneous plasmas and the effect these inhomogeneities have on the Thomson scattered spectrum.

\subsection{Thomson Scattering in Homogeneous Plasmas}

The calculation of the Thomson scattered shape factor is shown in appendix 4.2. This calculation treats each individual particle in the plasma as a test particle and calculates the electric field generated by the remaining particles which are shielding the test particle. The sum of the electric fields representing the shielding of each of the test particles, along with the electric field of the test particle electrons themselves, represent the electric field present in the plasma due to the thermal level density fluctuations. The spectral density fluctuations are then found simply by integrating the firstorder distribution function using the total electric field calculated from the test-particle approach. The scattered form factor obtained in this appendix is given by 


$$
\mathrm{s}(\overrightarrow{\mathrm{k}}, \omega)=\frac{2 \pi}{\mathrm{n}_{\mathrm{e}} \mathrm{k}}\left[\left|1-\frac{\chi_{\mathrm{e}}(\omega, \overrightarrow{\mathrm{k}})}{\varepsilon(\omega, \overrightarrow{\mathrm{k}})}\right|^{2} \mathrm{f}_{1 \mathrm{e}}(\omega / \mathrm{k})+\left|\frac{-\chi_{\mathrm{e}}(\omega, \overrightarrow{\mathrm{k}})}{\varepsilon(\omega, \overrightarrow{\mathrm{k}})}\right|^{2} \sum_{\alpha} \mathrm{z}_{\alpha}^{2} \mathrm{f}_{1 \mathrm{i} \alpha}(\omega / \mathrm{k})\right]
$$

where $\varepsilon(\omega, \overrightarrow{\mathrm{k}})=1+\chi_{\mathrm{e}}(\omega, \overrightarrow{\mathrm{k}})+\sum_{\alpha} \chi_{\mathrm{i} \alpha}(\omega, \overrightarrow{\mathrm{k}})$

The experiments performed for this dissertation involved primarily $\mathrm{CH}$ and Collodium plasmas. The $\mathrm{CH}$ plasmas had equal parts of Carbon and Hydrogen while the Collodium plasmas had $23.1 \%$ Carbon, $30.8 \%$ Hydrogen, $36.5 \%$ Oxygen, and 9.6\% Nitrogen. Figure 4.2.1 shows the scattering form factor, $S(\vec{k}, \omega)$, for a $\mathrm{CH}$ plasma assuming $k \lambda_{D e}=0.1$. The two peaks represent the scattering from ion acoustic waves present in the plasma. This figure shows how the Thomson scattering shape factor changes as the ratio of the ion to electron temperature changes.

Thomson scattering from electrostatic waves obeys the frequency and wavenumber matching conditions[88],

$$
\begin{aligned}
& \omega_{\text {scatt }}=\omega_{\mathrm{pr}} \pm \omega_{\mathrm{ia}} \text { and } \\
& \overrightarrow{\mathrm{k}}_{\text {scatt }}=\overrightarrow{\mathrm{k}}_{\mathrm{pr}} \pm \overrightarrow{\mathrm{k}}_{\mathrm{ia}}
\end{aligned}
$$

where $\omega_{\text {scatt }}\left(k_{s c a t t}\right), \omega_{p r}\left(k_{p r}\right), \omega_{i a}\left(k_{i a}\right)$ are the frequency (wavenumber) of the Thomson scattered wave, the probe beam, and the electrostatic wave respectively. These matching conditions are required for the waves to stay in phase with each other so that they may interact over a greater distance. In the case of ion acoustic waves, the ion acoustic frequency is much less than the 

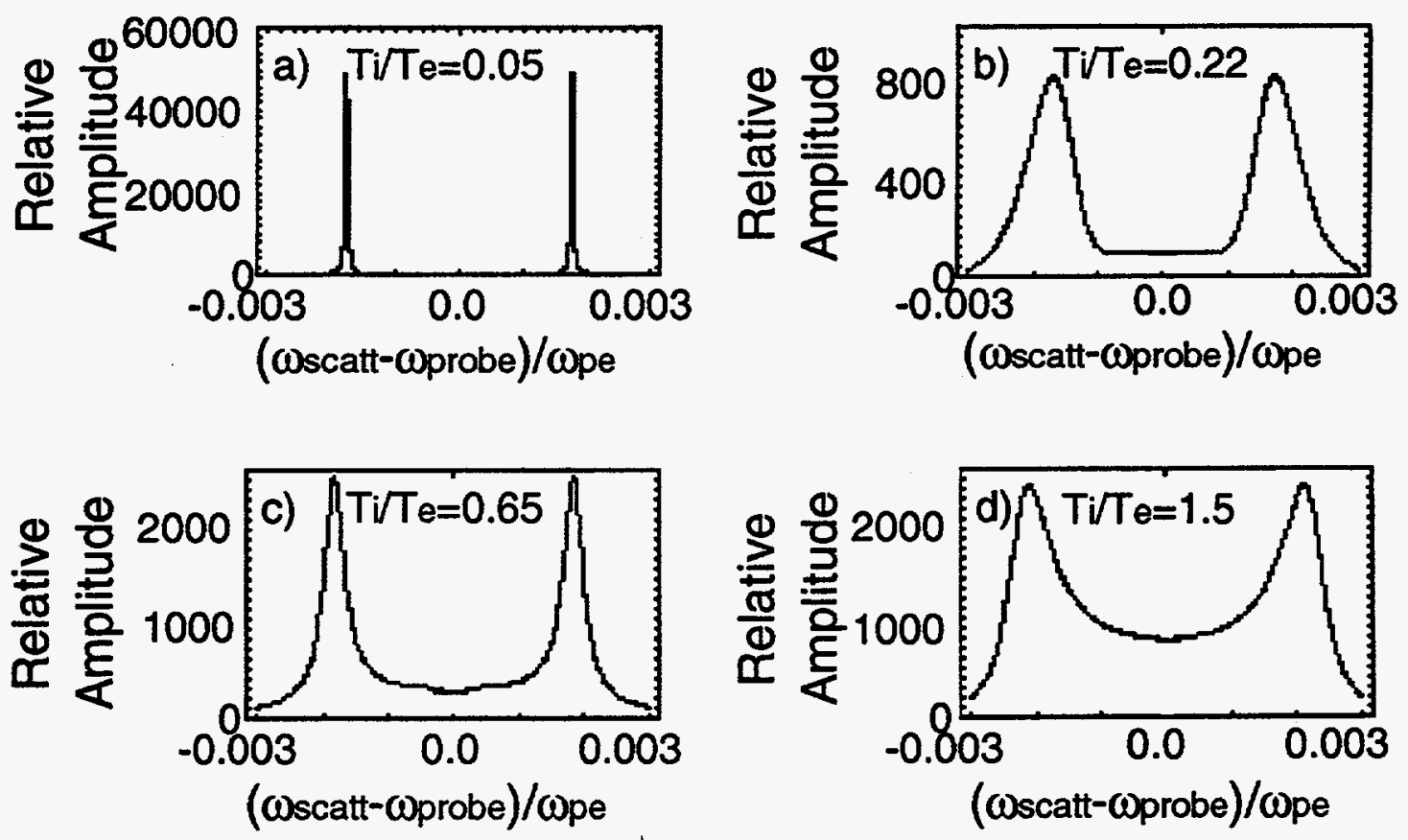

Figure 4.2.1 Thomson scattering form factor for a 50:50 $\mathrm{CH}$ plasma at various values of $T_{i} / T_{e}$. Figure 4.2.1a-d show the form factor at four different values of the ratio of the ion temperature to the electron temperature. In all four cases, the wavenumber multiplied by the electron Debye length is fixed at $\mathrm{k} \lambda_{\mathrm{De}}=0.1$ and the ions are assumed to be fully stripped. The vertical axis represents the amplitude of the scattered form factor, while the horizontal axis shows the form factor as a function of frequency. In all four cases the peaks are separated by twice the ion wave frequency which is approximately 0.002 wpe.

frequency of the electromagnetic waves and consequently the wavenumbers of the electromagnetic waves are approximately the same magnitude. Therefore, in an experiment the wavenumber of the ion acoustic waves, kia, 
being probed is determined primarily by the geometry of the Thomson scattering experiment[88],

$$
\left|k_{\mathrm{ia}}\right|=2\left|k_{\mathrm{pr}}\right| \sin \frac{\theta}{2}
$$

where $\mathrm{kpr}_{\mathrm{pr}}$ is the wavenumber of the Thomson probe beam and $\theta$ is the angle between the Thomson probe beam and the scattered electromagnetic wave.

The frequency separation between the scattered peaks is twice the ion wave frequency. This is proportional to the square root of the electron temperature, $T_{e}$, provided $k_{\mathrm{ia}} \lambda_{\mathrm{De}}<<1$ and may be expressed as

$$
\omega_{\text {sep }}=\alpha\left(\mathrm{T}_{\mathbf{i}}\right) \mathrm{k}_{\mathrm{ia}} \sqrt{\mathrm{T}_{\mathrm{e}}}
$$

where $\alpha\left(T_{i}\right)$ is a function of the ion temperature which must be calculated numerically from the scattering form factor, $s(\vec{k}, \omega)$, of the plasma. The relationship between the parameters of equation 4.2 .5 is demonstrated in figure 4.2.2 which shows the peaks of the Thomson scattered form factor. As the parameter $k_{i a} \lambda_{D e}$ is changed from 0.1 in $4.2 .2 \mathrm{a}$ to 0.2 in $4.2 .2 \mathrm{~b}$, the separation between the peaks doubles accordingly. The behavior of the separation as a function of $\mathrm{T}_{\mathrm{i}} / \mathrm{T}_{\mathrm{e}}$ remains unchanged, however, as stated in equation 4.2.5. The separation between the peaks is the primary feature which is used to determine the electron plasma temperature, $T_{e}$. The frequency of the Thomson scattered peak yields the ion wave frequency while the geometry of the experiment is used to determine the magnitude of $k_{i a}$, the wavenumber of the ion wave. The numerical solution of the Thomson shape 
factor is then used to infer the electron temperature. The primary source of uncertainty in the electron temperature is due to the unknown ion temperature which changes the separation between the peaks depending on its value relative to the electron temperature as shown in figure 4.2.2 a and $\mathrm{b}$.
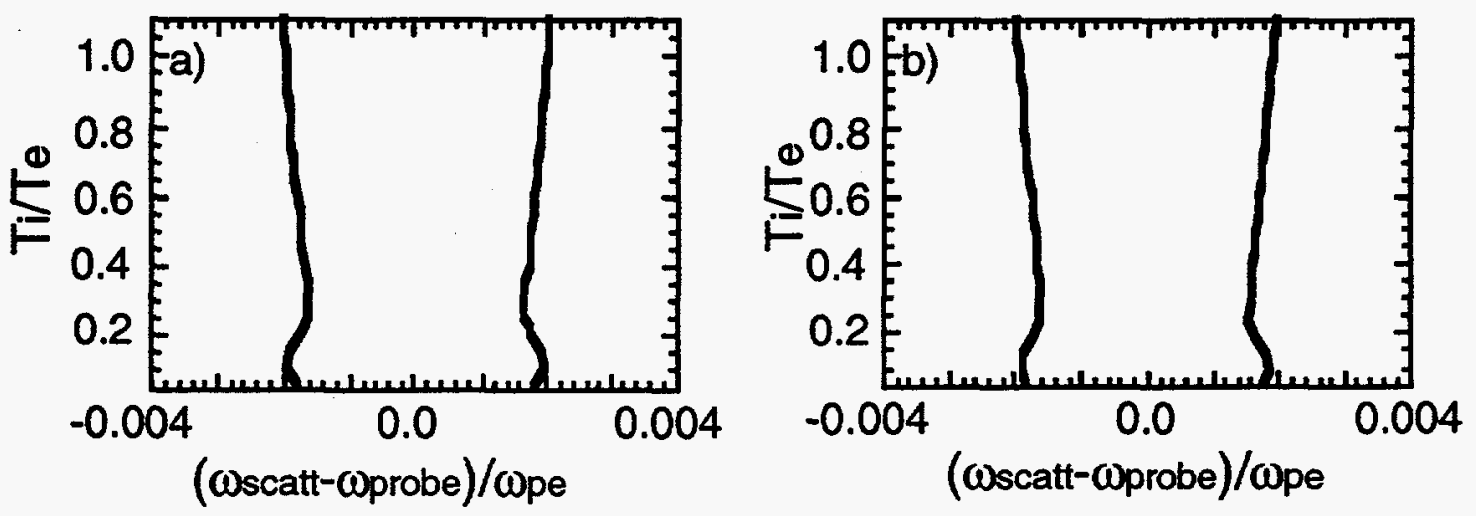

Figure 4.2.2 Dependence of the shape factor on the ratio of ion to electron temperature for a $\mathrm{CH}$ plasma. Figure 4.2.2a shows the maximum of the scattered peaks present in the form factor as a function of the ratio of the ion temperature to electron temperature with $k \lambda_{D e}=0.1$. Figure $4.2 .2 \mathrm{~b}$ again shows the maximum of the scattered peaks present in the form factor with $k \lambda_{D e}=0.2$ in this case.

Several of the attributes which are used to determine plasma parameters are shown in figure 4.2.3. Figure 4.2.3a shows the relative damping of the least damped ion wave mode in a $\mathrm{CH}$ plasma as a function of the ratio of $\mathrm{T}_{\mathrm{i}}$ to $\mathrm{T}_{\mathrm{e}}$. This represents the solution of the dispersion relation, $\varepsilon(\omega, \overrightarrow{\mathrm{k}})=1+\chi_{\mathrm{e}}(\omega, \overrightarrow{\mathrm{k}})+\sum_{\alpha} \chi_{\mathrm{i} \alpha}(\omega, \overrightarrow{\mathrm{k}})=0$. The graphical roots to the plasma 
dispersion function along with plots of the least damped modes can be seen in appendix 2.4 for several different ion species. Figure $4.2 .3 \mathrm{~b}-\mathrm{d}$ are taken
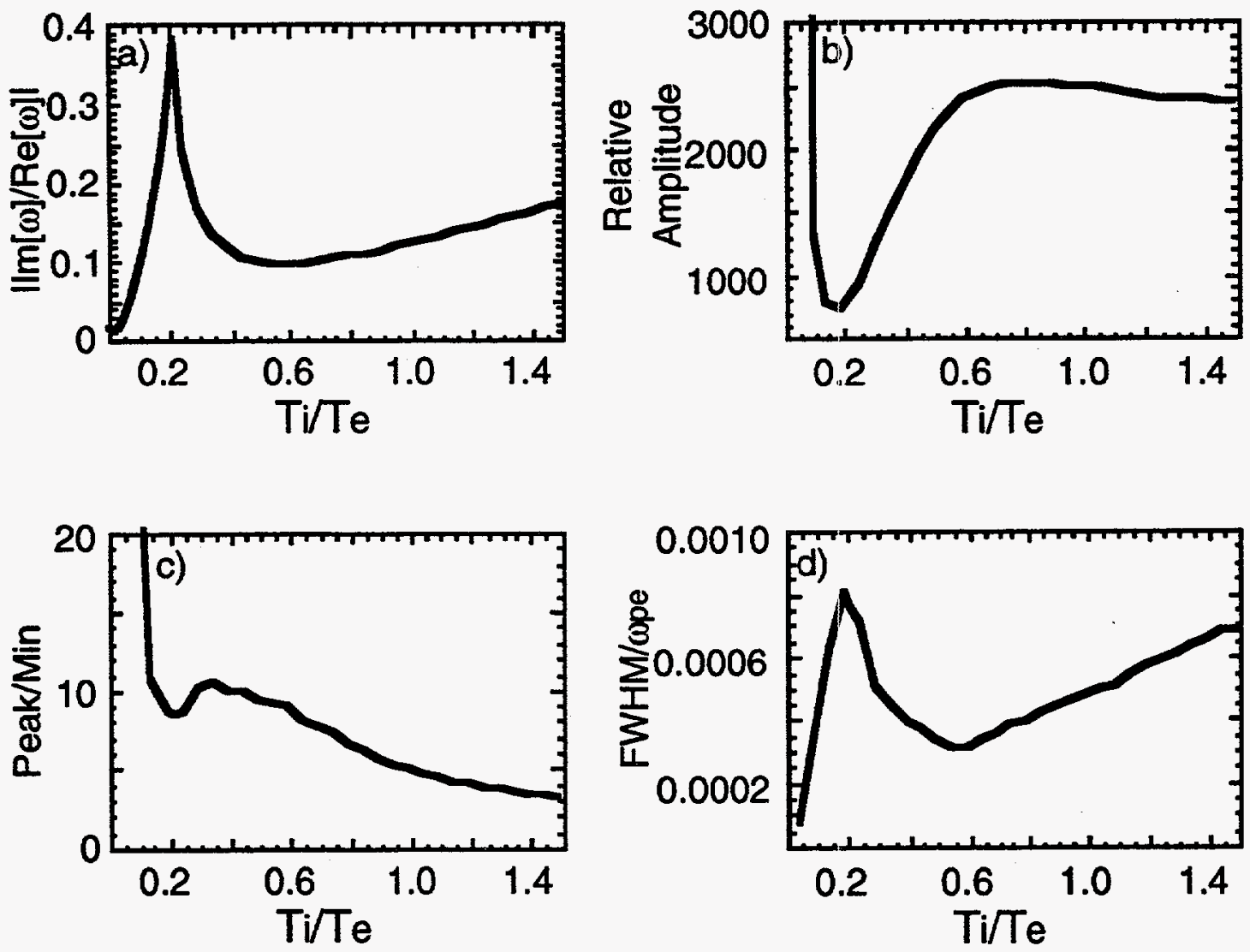

Figure 4.2.3 Attributes of the Thomson scattering shape factor, $S(k, \omega)$, for a $\mathrm{CH}$ plasma. The horizontal axis in figure 4.2.3 a-d represent the ratio of the ion to electron temperature present in the plasma. The vertical axis in figure 4.2.3a shows the relative damping of the ion wave expressed as the absolute magnitude of the imaginary component of the frequency divided by the real part of the frequency. The vertical axis in figure $4.2 .3 \mathrm{~b}$ represents the relative magnitude of the Thomson shape factor for a $\mathrm{CH}$ plasma. The vertical axis in figure $4.2 .3 \mathrm{c}$ represents the ratio of the peak to valley in the Thomson 
scattered shape factor and the vertical axis in figure 4.2.3d represents the frequency width of the Thomson scattered peaks normalized to the plasma frequency.

from the Thomson scattered form factor shown in figure 4.2.1 a-d. Figure 4.2.3b shows the relative magnitude of the scattered peaks as a function of the ratio of $T_{i}$ to $T_{e}$. Figure $4.2 .3 \mathrm{c}$ shows the ratio of the peak to valley as the ratio of $T_{i}$ to $T_{e}$ is varied. The valley is defined as the value of the shape factor where $\omega_{\text {scatt }}=\omega_{\text {probe }}$. Figure $4.2 .3 \mathrm{~d}$ shows the frequency width of the peaks in the Thomson scattered spectra as $T_{i} / T_{e}$ is varied. In all these figures, $k \lambda_{D e}$ is equal to 0.1 .

The ion temperature relative to the electron temperature is typically determined by the either the frequency width of the scattered peaks as depicted in figure $4.2 .3 \mathrm{~d}$ or by the ratio of the peak to valley in the Thomson scattered form factor as shown in figure $4.2 .3 \mathrm{c}$. Although these functions are typically monotonically increasing functions of the ratio of ion temperature to electron temperature for the frequency width and monotonically increasing for the case of the peak to valley measurement, in multi-species plasmas these features become multi-valued as is apparent in figure $4.2 .3 \mathrm{c}$ and $\mathrm{d}$. Both of these features follow qualitatively the damping of the ion acoustic waves as can be seen by comparing figure $4.2 .3 \mathrm{c}$ and $\mathrm{d}$ to figure 4.2.3 a.

As mentioned in section 2.1, when the wavelength of the ion wave becomes less than the shielding distance, the electron Debye radius, the electrons cannot completely shield the ion charge and hence the Thomson 
scattering signal from these waves is reduced. Figure 4.2 .1 a-d shows the Thomson scatter spectra for a $\mathrm{CH}$ plasma with $\mathrm{T}_{\mathrm{i}} / \mathrm{T}_{\mathrm{e}}$ maintained at 0.5 as a function of $k \lambda_{\text {De }}$. The magnitude of the Thomson shape factor decreases with increasing values of $k \lambda_{\text {De. }}$.
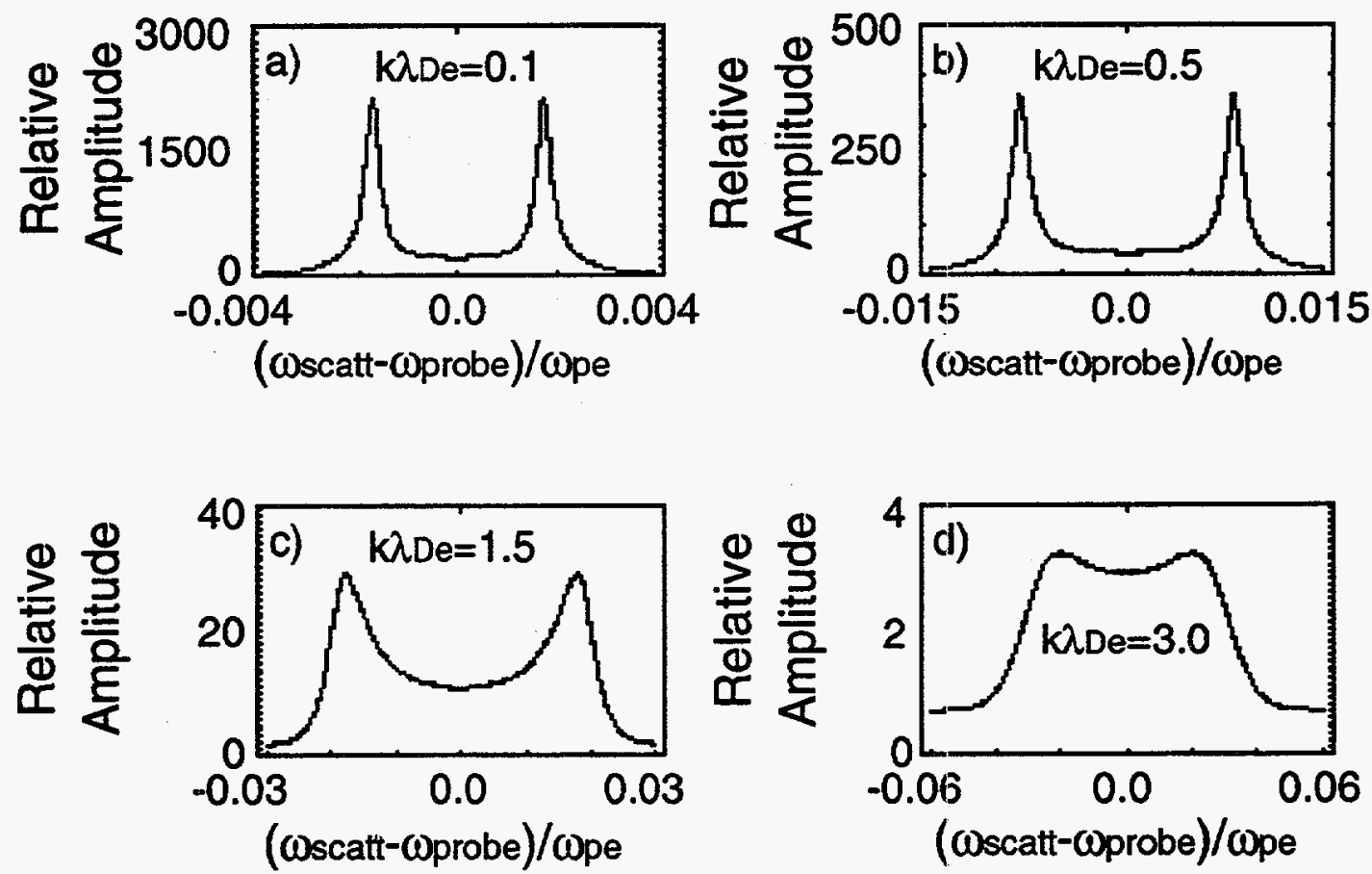

Figure 4.2.4 Thomson scatter spectra in a $\mathrm{CH}$ plasma as a function of $\mathrm{k} \lambda$ De with $\mathrm{T}_{\mathrm{i}} / \mathrm{T}_{\mathrm{e}}$ fixed at 0.5 . The vertical axes in all four figures represent the relative amplitude of the Thomson scattered signals while the horizontal axes are normalized frequency. Figure 4.2.4 a-d represent the Thomson shape factor with $k \lambda_{D e}=0.1, k \lambda_{D e}=0.5, k \lambda_{D e}=1.5$, and $k \lambda_{D e}=3.0$, respectively.

The Thomson scattering form factor can also be used to determine if there is a relative drift between the electrons and ions in the plasma. Figure 4.2.5 compares the Thomson scattering form factor for the case in which a 
Carbon plasma has no drift between the electrons and ions, $4.2 .5 \mathrm{a}$, to the case where the electrons are drifting with a relative drift of approximately 0.5 cs relative to the Carbon ions $4.2 .5 \mathrm{~b}$. If the electrons and ions are drifting relative to one another by an amount greater than the phase velocity of the ion wave, then the ion waves are unstable and may be driven to large amplitude. In multi-ion plasmas, there is also the possibility that there can be a drift between different ion species, which, in the case of low- $Z$ ion species where the damping is dominated by the ion distributions, can have a more important effect on the asymmetry of the peaks than a relative drift between the ions and electrons.
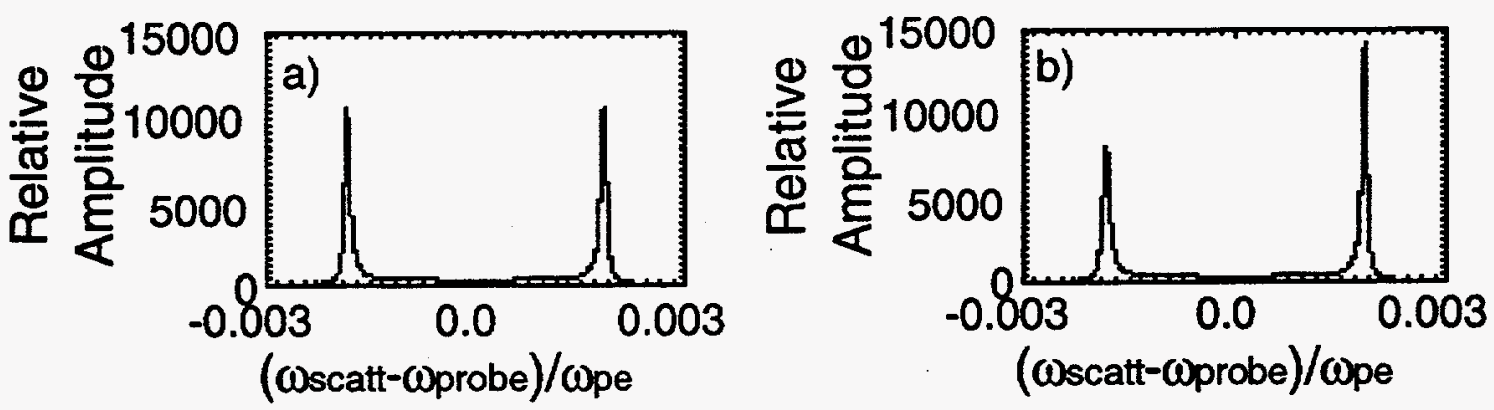

Figure 4.2.5 Thomson shape factor for a Carbon plasma in which there exists a finite drift between the electrons and ions. The horizontal axes shows the scattered frequency which the signal is scattered while the vertical axes show the relative amplitude. Figure 4.2.5a represents the case with zero drift between the electrons and ions. Figure $4.2 .5 \mathrm{~b}$ represents the case in which there is a relative drift between the ions and electrons of approximately 0.5 cs.

When measuring the plasma temperature, it is important to minimize the angular spread in the Thomson probe beam and collection optics to 
reduce the uncertainty in the magnitude of ion wavenumber being probed. The Thomson scattering data is typically fit to the scattering form factor to extract the electron temperature, the ion temperature, and the relative drift between the electrons and the ions. Although in a homogeneous plasma, a fit can be made between the width of the Thomson scattered peaks and their separation to determine the ion and electron temperature, this is much more difficult in an inhomogeneous plasma. In inhomogeneous laser-produced plasmas, the flow gradient scalelengths are typically on the order of the spot size of the probe beam. This causes the probe to effectively see a range of flow velocities which are much broader than the homogeneous FWHM of the scattered peaks. Due to differences in the temperature gradients, the probe beam can also sample a range of temperature ratios. These flow gradients and temperature gradients can make the measurement of $T_{i}$ difficult. The flow gradients can significantly broaden the spectrum making any estimation of $T_{i}$ from the width of the scattered peaks meaningless. Instrumental effects and flow gradients can also fill in the valley of the Thomson shape factor relative to the maximum of the scattered peaks causing an overestimation of the ratio of ion to electron temperature present in the plasma.

A simple criteria can be established for the validity of using the homogeneous FWHM of the Thomson scattered spectra. The dispersion relation for an ion acoustic wave in an inhomogeneous plasma is given by

$$
\omega_{i a}=\vec{k}_{i a} \cdot \vec{u}_{f}\left(1-\frac{x}{L_{v}}\right)+c_{s} k_{i a}
$$


where $\omega_{\mathrm{ia}}\left(\mathrm{k}_{\mathrm{ia}}\right)$ are the frequency(wavenumber) of the ion acoustic wave and $c_{s}, u_{f}, L_{V}$ are the sound speed, the flow velocity, and the flow velocity scalelength respectively. $L_{V}$ can be defined as $c_{S}\left(d u_{f} / d x\right)^{-1}$. As a rough approximation, the ion acoustic frequency changes by an amount equal to it's value at $x=0$ over a distance approximately equal to the flow velocity scalelength. The FWHM of the scattered peaks is roughly equal to the damping of the ion acoustic wave divided by the ion acoustic frequency. Therefore, in most cases the focal spot of the probe beam divided by the flow velocity scalelength must be less than the damping on the ion acoustic wave divided by the ion acoustic frequency in order for a measurement of the FWHM of the spectra to yield useful results. For most small-scale laserplasma experiments this is not the case, however, for large-scale laser-plasma experiments such as found in exploding foil targets, gas jet experiments, backfilled chambers, or gas bag experiments, flow velocity scalelengths of over a thousand wavelengths can exist. In this case the broadening due to flow velocity inhomogeneity could be a small percentage of the broadening due to damping on the ion wave itself, particularly in the case that there is heavy damping on the ion wave. For high- $Z$ ion species where the damping is a few percent of the ion wave frequency, the probe beam area would have to be close to one percent of the flow velocity scalelength which is unlikely even for many large-scale plasmas. The above criteria assumes that the scattering occurs uniformly throughout the focal spot size of the probe beam. Narrowing can occur due to the distribution of intensities in the focal spot(hot spots) and due to the dependence on density present in the scattering formula. Improvements are made by going to larger scalelength plasmas and 
smaller focal spot diameters and higher $f$ numbers for the Thomson probe beam.

\subsection{Thomson Scattering in Inhomogeneous Plasmas}

All of the calculations presented in the section above and in the relevant appendices have assumed a homogeneous plasma. In laser-produced plasmas, however, inhomogeneities are very important in limiting the amplitude which the collective Thomson scattered signals are driven. In the homogeneous case, the coherent peaks in the form factor are determined by the damping on the electrostatic waves which are scattering the Thomson probe beam. When inhomogeneity is included, the amplitude of the coherent peaks can be determined by the plasma inhomogeneities rather than the damping on the electrostatic wave, particularly in the case of Langmuir waves. The incoherent part of the spectrum is not affected by the inhomogeneity. This can change the peak to valley in the scattered form factor, as well as the full width half maximum of the scattered peaks. More importantly, the broad range of flow velocities sampled by the probe beam can cause a large broadening of the scattered peaks making any estimation of the width of the scattered peaks meaningless.

A model can be constructed that shows the electromagnetic wave amplitude produced by the Thomson scattering of a electromagnetic wave from a fixed amplitude electrostatic wave in an inhomogeneous plasma[ $[7,27,80]$. This model is useful in predicting the magnitude of the signal 
generated from Thomson upscattering off of Langmuir or ion waves when this is not a stimulated process. Stimulated scattering is then just a special case of collective Thomson scattering. The vector potential of the scattered light wave, $A_{s c}$, is driven due to the transverse current formed by the coupling of the incident electromagnetic wave, Apr, and the ion acoustic wave, $n_{\text {epw }} / n_{0 e}$, which is given by

$$
\left(\frac{\partial^{2}}{\partial t^{2}}+\omega_{p e}^{2}-c^{2} \frac{\partial^{2}}{\partial x^{2}}\right) \frac{q A_{s c}(x)}{m_{e} c^{2}}=-\omega_{p e}^{2} \frac{n_{e p w}(x)}{n_{o e}} \frac{q A_{p r}(x)}{m_{e} c^{2}}
$$

where $\omega_{\mathrm{pe}}$ is the plasma frequency, $\mathrm{c}$ is the speed of light, $\mathrm{m}_{\mathrm{e}}$ and $\mathrm{q}$ are the mass and charge of an electron respectively. The wave amplitudes can be expressed in the form $\frac{\mathrm{qA}_{\alpha}(\mathrm{x})}{\mathrm{m}_{\mathrm{e}} \mathrm{c}^{2}}=\frac{1}{2} \Psi_{\alpha}(\mathrm{x}) \exp \left[\mathrm{i}\left(\int \vec{k}_{\alpha} \cdot \mathrm{d} \overrightarrow{\mathrm{x}}-\omega_{\alpha} \mathrm{t}\right)\right]+$ c.c.. Equation 4.3.1 is reduced to a first order differential equation using the slowly varying envelope assumption that the wave amplitudes are slowly varying with respect to the wavenumber, $k_{\alpha}$, and frequency, $\omega_{\alpha}$, of the wave, or $\mathrm{k}_{\alpha} \Psi_{\alpha}(\mathrm{x}) \ll<\frac{\mathrm{d} \Psi_{\alpha}(\mathrm{x}, \mathrm{t})}{\mathrm{dx}}$, and $\omega_{\alpha} \Psi_{\alpha}(\mathrm{x}) \ll \frac{\mathrm{d} \Psi_{\alpha}(\mathrm{x}, \mathrm{t})}{\mathrm{dt}}$. In the case of ion acoustic waves of amplitude $\Psi_{\mathrm{ia}}$ in a linear profile, the steady state form of equation 4.3.1 can be written as

$$
\frac{\mathrm{d} \Psi_{\mathrm{sc}}}{\mathrm{dx}}=\frac{\omega_{\mathrm{pe}}^{2}\left(\hat{\mathrm{e}}_{\mathrm{pr}} \cdot \hat{\mathrm{e}}_{\mathrm{sc}}\right)}{4 \mathrm{ic}^{2} \mathrm{k}_{\mathrm{sc}}}\left(\Psi_{\mathrm{ia}} \Psi_{\mathrm{pr}}\right) \exp \left[\frac{\mathrm{i} \kappa^{\prime} \mathrm{x}^{2}}{2}\right]
$$

where the wavenumber mismatch, $\left(\vec{k}_{s c}(x)-\vec{k}_{p r}(x) \pm \vec{k}_{i a}(x)\right) \cdot d \vec{x} \approx \kappa^{\prime} x$, has been Taylor expanded about the perfect phase matching point. Contour 
integration can be used to obtain the magnitude of the scattered vector potential as shown in Appendix 4.5. The resultant scattered vector potential, assuming a linear phase mismatch, is

$$
\left|\Psi_{\mathrm{sc}}\right|=\frac{\sqrt{2 \pi}}{\sqrt{\kappa^{\prime}} 4} \frac{\omega_{\mathrm{pe}}^{2}\left(\hat{\mathrm{e}}_{\mathrm{pr}} \cdot \hat{\mathrm{e}}_{\mathrm{sc}}\right)}{\mathrm{c}^{2} \mathrm{k}_{\mathrm{sc}}}\left|\Psi_{\mathrm{ia}}\right|\left|\Psi_{\mathrm{pr}}\right| .
$$

Figure 4.3.1a shows the amplitude of the scattered vector potential from a plasma containing a linear phase mismatch. This situation is found when Thomson scattering from Langmuir waves in a linear profile and in a parabolic profile when the waves are not at the top of the parabola. The initial conditions are that at $t=0$, the amplitude of the scattered vector potential is zero everywhere is space. In this figure, the Thornson scattered signal has reached the saturation amplitude in figure 4.3.1 a and the signal is traveling to the left at the group velocity in figures $4.3 .1 \mathrm{~b}$ and $4.3 .1 \mathrm{c}$. The dashed line in figure $4.3 .1 \mathrm{c}$ shows the amplitude of the steady state signal calculated in equation 4.3.3 above. Figure 4.3.1 d shows the growth of the scattered vector potential at the perfect phase matching point, $x=0$. This graph shows the amplitude growing linearly in time at approximately the homogeneous growth rate, $\Psi_{\text {scatt }}=$ t $\omega_{\text {pe }}^{2} \Psi_{\text {probe }} \Psi_{\text {epw }} / 4 \omega_{\text {s }}$ with $\omega_{\text {pe }}^{2} \Psi_{\text {probe }} \Psi_{\text {epw }} / \omega_{\text {s }}=1$ used in the simulation.

In the case of Thomson downscattering, the scattering process can be a stimulated process known as stimulated Brillouin. scattering, provided the square of the growth rate for stimulated Brillouin scattering is greater than the product of the damping on the daughter waves[16]. In this case the Thomson downscattered peak can be driven higher than the upscattered 
peak, causing an asymmetry in the Thomson scattered peaks. This asymmetry can also be caused by a relative drift between the ions and electrons which can
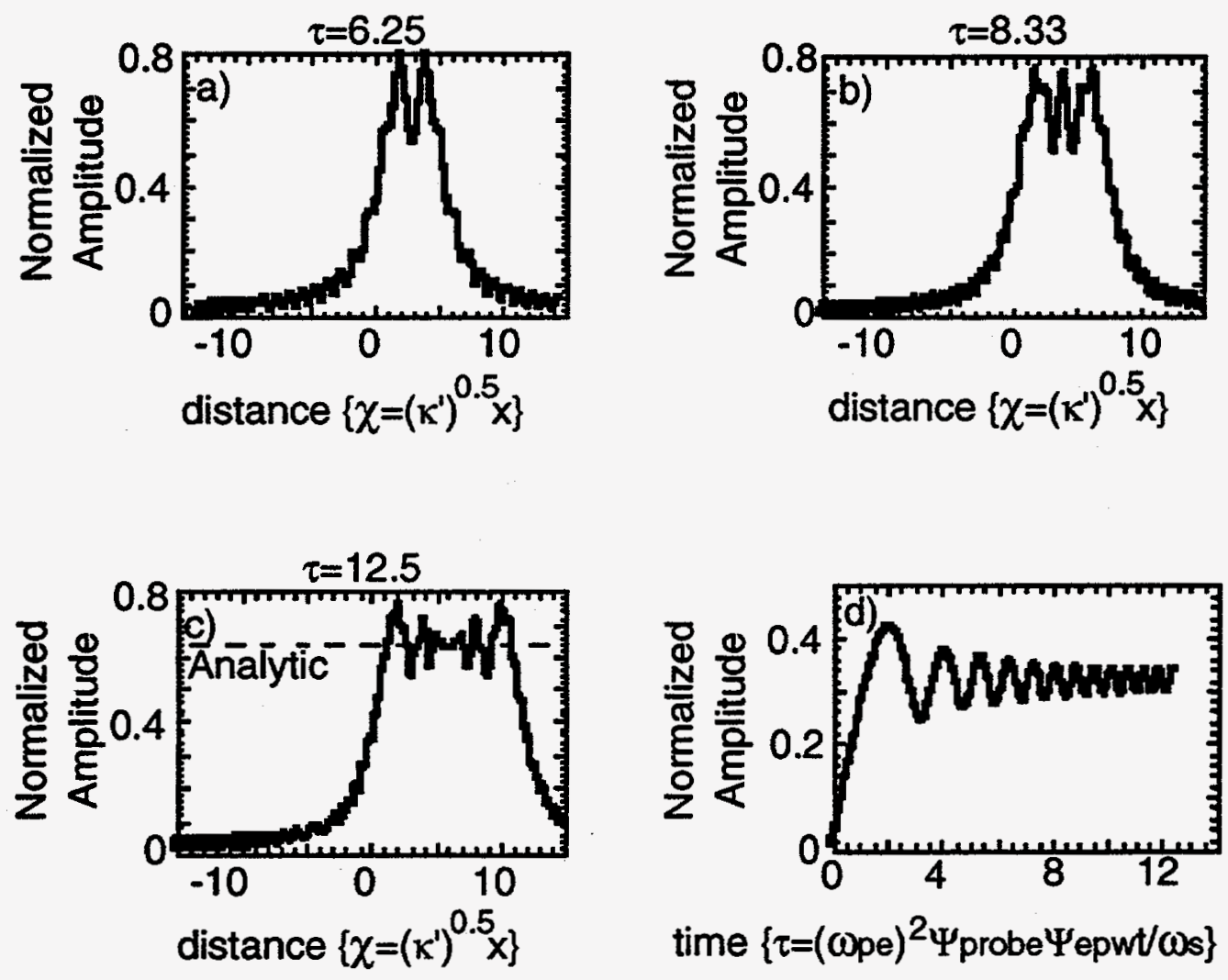

Figure 4.3.1 Numerical evaluation of the Thomson scattered vector potential for the case of a linear phase mismatch between the interacting waves. The scattered vector potential as a function of distance is shown in figure $4.3 .1 \mathrm{a}-\mathrm{c}$ each representing a different time. The vertical axis on all of these graphs represents the amplitude of the Thomson scattered vector potential. The horizontal axis on figures a-c represent the distance along the profile with $\mathrm{x}=0$ representing the perfect phase matching point. The horizontal axis in figure 4.3.1 d represents time. 
lead to asymmetric Landau damping on the ion waves, as discussed above. In fact if the drift velocity between the ions and electrons is greater than the phase velocity of the ion waves, the ion waves become unstable. The majority of the thermal Thomson scattering spectra found in the literature have interpreted any asymmetry in the peaks as caused by a relative drift between the ions and electrons, however, in some of these cases the Thomson probe intensity is above the homogeneous threshold for stimulated Brillouin scattering. Using the results in chapter 2 from the convective amplification section, the absolute magnitude of the square of the downscattered vector potential can be written as

$$
\left|\Psi_{s c}\right|^{2}=\left(\frac{V_{\text {gion }}}{V_{d s}}\right) \sqrt{\frac{Z T_{e}}{M_{i}}} \frac{\omega_{p e}^{2}\left(\hat{e}_{p r} \cdot \hat{e}_{s c}\right) M_{i}}{\omega_{s} c^{2} k_{i a} Z m_{e}}\left|\Psi_{e p w}\right|^{2}\left[e^{G_{s s s}}-1\right] .
$$

When the gain for stimulated Brillouin scattering is small, the exponential may be expanded and equation 4.3.4 reduces to equation 4.3.3. The ratio of the stimulated downscattered feature, $\left|\Psi_{\text {sc }}\right|_{\text {down' }}^{2}$ to that of the Thomson upscattered feature, $\left|\Psi_{\mathrm{sc}}\right|_{\mathrm{up}}^{2}$, is

$$
\frac{\left|\Psi_{\text {sc }}\right|_{\text {down }}^{2}}{\left|\Psi_{\text {sc }}\right|_{\text {up }}^{2}}=\frac{e^{G_{\text {sss }}}-1}{G_{\text {sbs }}}
$$

where $G_{s b s}$ is the Rosenbluth gain defined in chapter 2, section 2.2. This is then another mechanism which can lead to an asymmetry in the Thomson scattered peaks. This process could possibly be used as a diagnostic to determine the "local" flow gradient scalelength, the scalelength within the 
area of the probe beam, due to the linear dependence on $L_{V}$ contained in $G_{s b s}$. The primary problem with this diagnostic would be the difficulty in monitoring the speckle pattern of the intensity present in the probe beam, as well as an uncertainty in the plasma frequency. The flow velocity and sound speed, at least, would be known due to the location of the peaks relative to the probe frequency and relative to each other, respectively. 


\section{Chapter 5}

\section{Initial Thomson scattering experiments; verification of Raman scattering models}

\subsection{Introduction}

In this experiment, Thomson scattering was used to measure the Langmuir wave spectrum driven by stimulated Raman scattering. These measurements provided a direct comparison between stimulated Raman scattering and the enhanced Thomson scattering model for the electromagnetic emission between $\omega_{0}$ and $\omega_{0} / 2$ observed in laser-produced plasmas. The Thomson scattering measurements showed that the Langmuir wave spectrum resulted from stimulated Raman scattering and not from enhanced Thomson scattering. The width in k-space of the measured Thomson scattering signals also has implications for the saturation of the Langmuir waves.

Many experiments have measured the scattered electromagnetic wave associated with stimulated Raman scattering and observed features which are difficult to explain using linear theory[21,55]. 
In these experiments stimulated Raman scattering was often observed at levels significantly above the spectral intensity calculated assuming convective amplification from thermal noise levels. Another feature observed in many of the experiments is a gap in the Raman spectra, discussed in section 2.8, extending from close to the quarter critical surface to somewhere below twenty percent of the critical density. In addition, the spectral intensity in these experiments typically peaked at long wavelengths, corresponding to lower densities, even though linear convective theory would predict higher gain with increasing density, providing the density scalelength does not change. These discrepancies with linear theory have led to several hypotheses concerning the Raman spectrum observed in experiments[21,39].

One of the hypotheses used to explain the discrepancies cited above was the "enhanced Thomson scattering" model[21]. This model assumes that hot electron pulses moving through the plasma excite large level electron plasma waves via the bump-on-tail instability. The electron distribution in such a case is shown in figure 5.1.1. The Langmuir waves whose phase velocity falls on the reversed side of the hot electron distribution, as drawn in gray in figure 5.1.1, are unstable and amplify at the appropriate growth rate. These waves are unstable because those electrons traveling close to the phase velocity of a particular wave experience an almost constant potential from the wave. When there are more particles traveling slightly faster than the wave, then the wave gains energy from the electrons. On the other hand, when there are more particles traveling slightly slower than the phase velocity of the wave, the particles gain energy from the wave and the wave damps. As 
shown in figure 5.1.1, the Langmuir waves whose phase velocity fall on the reversed side of the hot electron distribution have more electrons traveling slightly faster than the phase velocity of the waves and therefore these waves experience a net amplification at the expense of the hot electrons.

The scattered electromagnetic wave measured in experiments would then result from Thomson scattering of the pump beam from these enhanced electron plasma waves. The authors proposed that the hot electrons could originate from the two plasmon decay instability near the quarter critical surface. In the case of stimulated Raman scattering, the wavevectors of the electron plasma waves being scattered from travel in the same direction as the incident laser or probe beam. For solid target experiments, this requires that the beam of electrons leaving the quarter critical surface travel down the density gradient to a sheath region in the plasma and reflect. The reflected electrons then travel up the density gradient where they can excite Langmuir waves which the probe beam can scatter from, producing the backscattered electromagnetic wave observed in experiments.

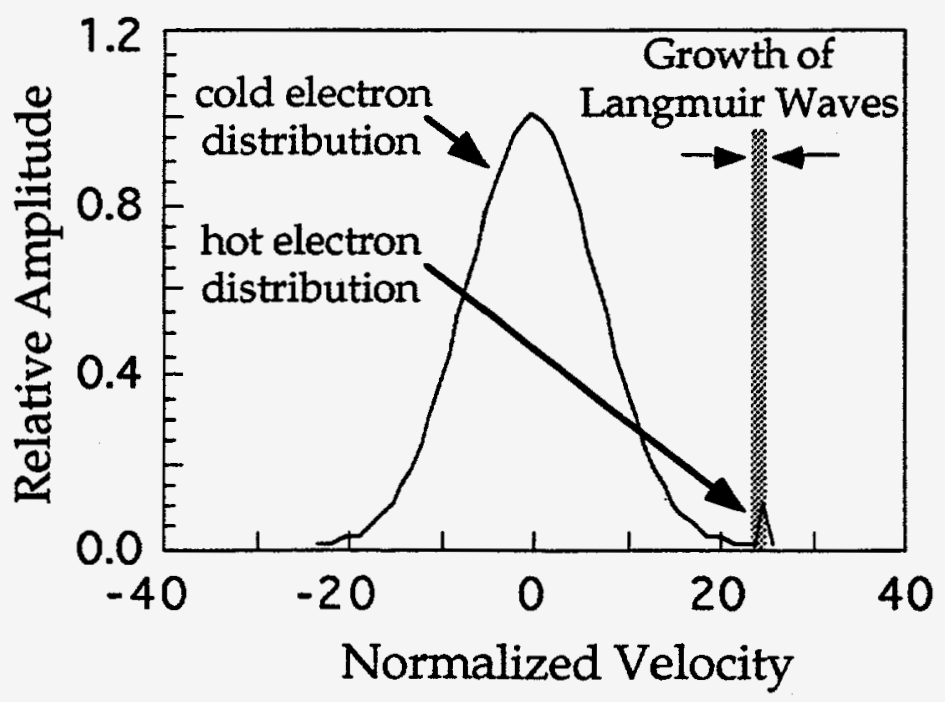


Figure 5.1.1 Model distribution of the electrons in a plasma containing a hot electron distribution. In this case, the distribution contains a cold Maxwellian component and also a hot electron distribution with a normalized velocity centered at 25 .

This experiment was designed to look at the Langmuir wave spectrum in an attempt to distinguish between the enhanced Thomson scattering model and stimulated Raman scattering[40]. In the next section, the experimental setup is presented along with the plasma parameters present during the experiment. Thomson scattering is discussed following the experimental description. The measured spectrum observed in the experiments is discussed following the experimental setup and these observations are compared to stimulated Raman scattering and the enhanced Thomson scattering model.

\subsection{Experimental description}

The experimental setup is shown in figure 5.2.1. The plasma was formed by a $1.064 \mu \mathrm{m}, 2.5 \mathrm{~ns}$ FWHM Nd:Glass laser. The focal spot of the interaction beam was varied between 80 to $400 \mu \mathrm{m}$ in diameter and the laser energy ranged from 100 to 160 Joules, resulting in average intensities on target between $3 \times 10^{13}$ and $1 \times 10^{15} \mathrm{~W} / \mathrm{cm}^{2}$. The targets consisted of Collodium thin foils mounted on $5 \mathrm{~mm}$ diameter rings with thicknesses ranging between 100 and $500 \mathrm{~nm}$, as well as solid Carbon targets which retained a critical surface for the entire duration of the interaction beam. 
The Thomson probe beam was formed by taking ten percent of the energy from the $1.064 \mu \mathrm{m}$ interaction beam. This energy was then frequency upconverted, resulting in a $355 \mathrm{~nm}$ Thomson probe beam with a temporal duration slightly less than $1.5 \mathrm{~ns}$. The Thomson probe beam was focused using an $f / 10$ lens which was masked to approximately $f / 100$ giving the probe beam an angular spread of less than 0.6 degrees. The probe beam was incident at 63 degrees from the interaction beam such that the Langmuir waves corresponding to Raman backscattering could be observed.

The Thomson scattering diagnostic, used to measure the Langmuir wave spectrum, is shown in Figure 5.2.1. The Thomson scattered light was collected over a range from 15 degrees to approximately 40 degrees from the probe beam. This was the angular range corresponding to the Thomson scattered signal from the Langmuir waves responsible for Raman backscatter of the incident beam. This scattered light was sent to a spectrometer where the Fourier transform plane was imaged onto the entrance slit of the spectrometer. Because the spectrometer was a 1:1 imaging device, the angular information focused on the entrance slit of the spectrometer was also imaged at the output of the spectrometer. This allowed for the angular measure in one direction and wavelength resolution in the perpendicular direction. At the exit plane of the spectrometer, an S-20 Kentech gated optical imager(GOI) was used to record the wavelength versus angle of the Thomson scattered signal. The GOI served two purposes. The microchannel plate amplified the Thomson scattered signal and the gate allowed the Thomson scattered signal to be discriminated from the Bremsstrahlung emission which although had much less intensity, it occurred over a much longer period of time. The 
output phosphor on the GOI was imaged onto a CCD camera which recorded each shot. Ultimately the CCD camera recorded wavelength versus angle of the Thomson scattered light emitted from the plasma. Assuming a resonant scattering process, the angle which the light exits the plasma can be used to determine the wavelength of the Langmuir wave from which the probe beam scatters. This diagnostic then provides a direct measurement of $\omega$ versus $k$ of the Langmuir waves corresponding to resonant scatter from the probe beam.

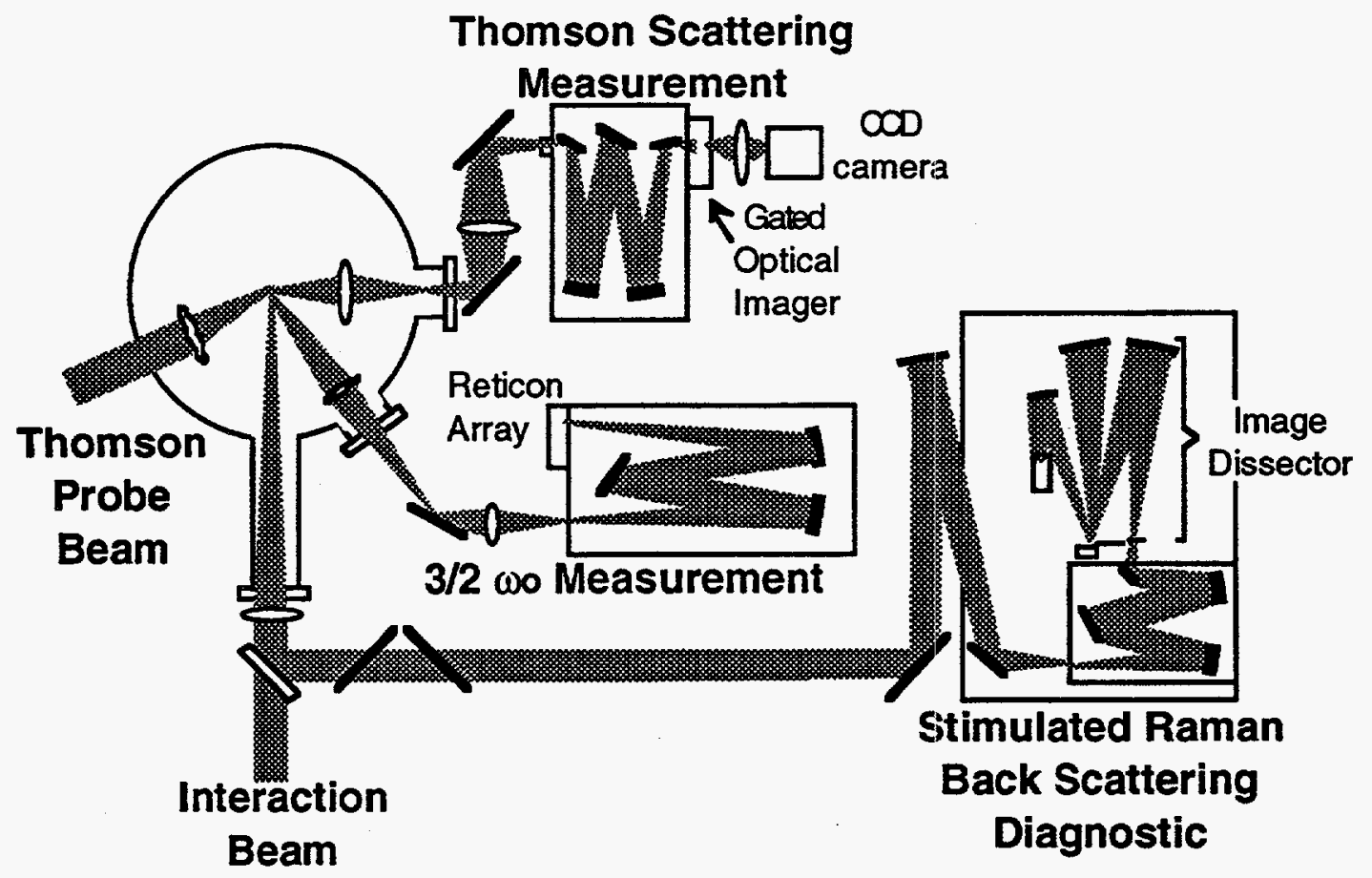

Figure 5.2.1 Experimental layout showing the interaction and probe beams, along with the interaction chamber. This figure also shows the three primary diagnostics used in this experiment; the Thomson scattering diagnostic, the stimulated Raman backscattering measurement, and the diagnostic used to measure the $3 / 2 \omega_{0}$ emission from the plasma. 
The Raman backscatter diagnostic, used to measure the backscattered light above $1.064 \mu \mathrm{m}$, is also shown in Figure 5.2.1. This diagnostic measured the light which was backscattered into the focusing lens. A glass plate was placed in the incident beamline which reflected approximately eight percent of the backscattered Stimulated Raman scattered light. This reflected light was focused onto the entrance slit of a Czurney-Turner spectrometer which was used to spectrally resolve the signal. The spectrometer had an $85 \mathrm{gr} / \mathrm{mm}$ grating and the focusing mirrors had a radius of curvature of $0.25 \mathrm{~m}$. The output plane of the spectrometer formed the input into an image dissector which was used to obtain a time integrated spectrum of backscattered light of wavelengths greater than $1.064 \mu \mathrm{m}[94]$. The output of the spectrometer provided a continuous frequency versus position measurement which the image dissector then turned into a sequence of discrete frequency bands versus time at its output. The output of the image dissector was focused onto a Au:Ge detector which provided a time integrated signal in discrete frequency bands. The output of the gold germanium detector was sent to an oscilloscope and recorded on film. Figure 5.2.2 shows a schematic of the detector circuit. The Au:Ge detector was cooled with liquid Nitrogen to $77^{\circ} \mathrm{K}$.

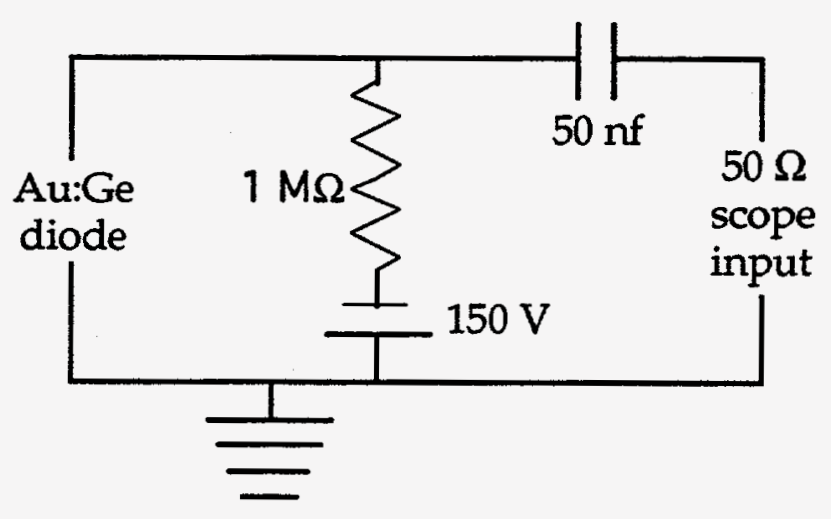


Figure 5.2.2 Schematic of the detector circuit used for the Au:Ge detector. The $50 \Omega$ input from the oscilloscope, in conjunction with the $70 \mathrm{pf}$ junction capacitance of the Au:Ge detector, gave an RC decay time of approximately 3.5 ns.

The oscillator for the interaction beam was used to calibrate the loss of signal as a function of round trips in the image dissector. This method was also used to determine the wavelength range contained in each wavelength band sent to the detector. Figure 5.2.3 shows the attenuation as a function of round trip passes inside the image dissector. The time between signals is approximately $13 \mathrm{~ns}$ and the decay time for an individual signal is approximately $3.5 \mathrm{~ns}$. Each signal represents a frequency bin of approximately $55 \mathrm{~nm}$.

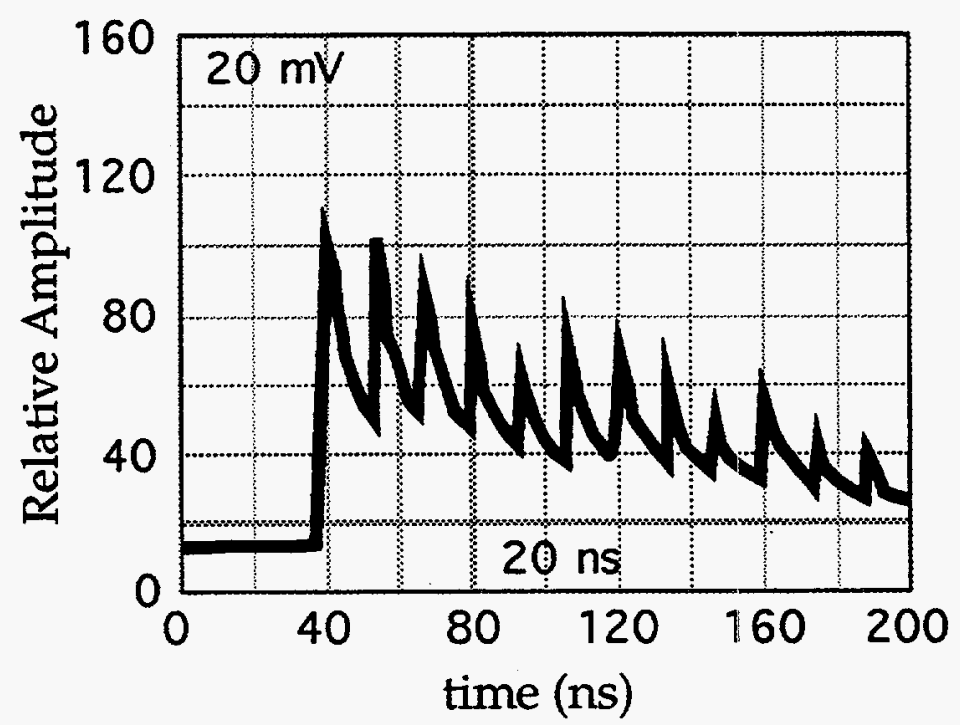

Figure 5.2.3 Signal attenuation as a function of round trip passes through the image dissector. 
The $3 / 2 \omega_{0}$ emission driven during these experiments was measured using a spectrometer and a Reticon array as shown in Figure 5.2.1. As explained in chapter 3 , the $3 / 2 \omega_{0}$ emission results from Thomson scattering of the incident pump beam from Langmuir waves produced by two plasmon decay near the quarter critical surface[76]. The image plane of the plasma was focused onto the slit of the spectrometer, and the spectrally dispersed light was then focused onto a reticon array providing a time integrated measurement of the spectral intensity near $709 \mathrm{~nm}$.

\subsection{Collective Thomson scattering}

Collective Thomson scattering was the primary diagnostic used in this experiment. The Thomson scattering diagnostic was used to obtain the spectrum of Langmuir waves which had been driven by the interaction beam. Resonant Thomson scattering satisfies the frequency and wavenumber matching conditions $\omega_{\text {scatt }}=\omega_{\mathrm{pr}} \pm \omega_{\mathrm{ia}}$ and $\overrightarrow{\mathrm{k}}_{\mathrm{scatt}}=\overrightarrow{\mathrm{k}}_{\mathrm{pr}} \pm \overrightarrow{\mathrm{k}}_{\mathrm{ia}}$ where $\mathrm{k}_{\text {scatt }}\left(\omega_{\text {scatt }}\right), \mathrm{k}_{\mathrm{pr}}\left(\omega_{\mathrm{pr}}\right)$, and $\mathrm{k}_{\mathrm{ia}}\left(\omega_{\mathrm{ia}}\right)$ are the wavenumber(frequency) of the Thomson scattered wave, the probe beam, and the ion acoustic wave, respectively. Through these matching conditions, the frequency and angle that the Thomson scattered signal is observed determines the frequency and wavevector of the Langmuir wave being probed.

In this experiment, Thomson scattering was used to examine the spectrum of Langmuir waves driven in an inhomogeneous plasma. A model can be constructed which gives the electromagnetic wave amplitude produced by the Thomson scattering of a electromagnetic wave from a fixed amplitude 
Langmuir wave in an inhomogeneous plasma[7,27,80]. This model is useful in predicting the magnitude of the signal generated from Thomson scattering off of Langmuir waves whose amplitude has saturated. This model is also useful in determining the amplitude of the scattered vector potential present in the enhanced Thomson scattering model. The vector potential of the scattered light wave, $A_{s c}$, is driven due to the transverse current formed by the coupling of the incident electromagnetic wave, $\mathrm{A}_{\mathrm{pr}}$, and the Langmuir wave, $n_{\text {epw }} / n_{\mathrm{oe}}$, which is given by

$$
\left(\frac{\partial^{2}}{\partial t^{2}}+\omega_{p e}^{2}-c^{2} \frac{\partial^{2}}{\partial x^{2}}\right) \frac{q A_{s c}(x)}{m_{e} c^{2}}=-\omega_{p e}^{2} \frac{n_{e p w}(x)}{n_{o e}} \frac{q A_{p r}(x)}{m_{e} c^{2}}
$$

where $\omega_{\mathrm{pe}}$ is the plasma frequency, $\mathrm{c}$ is the speed of light, $\mathrm{m}_{\mathrm{e}}$ and $\mathrm{q}$ are the mass and charge of an electron respectively. The wave amplitudes can be expressed in the form $\frac{\mathrm{qA}_{\alpha}(\mathrm{x})}{\mathrm{m}_{\mathrm{e}} \mathrm{c}^{2}}=\frac{1}{2} \Psi_{\alpha}(\mathrm{x}) \exp \left[\mathrm{i}\left(\int \overrightarrow{\mathrm{k}}_{\alpha} \cdot \mathrm{d} \overrightarrow{\mathrm{x}}-\omega_{\alpha} \mathrm{t}\right)\right]+\mathrm{c}$.c. [27].

Equation 5.3.1 is reduced to a first order differential equation under the assumption that the wave amplitudes are slowly varying with respect to the wavenumber and frequency of the wave, or $k_{\alpha} \Psi_{\alpha}(x) \ll<\frac{d \Psi_{\alpha}(x, t)}{d x}$, and $\omega_{\alpha} \Psi_{\alpha}(x) \ll \frac{d \Psi_{\alpha}(x, t)}{d t}$. In the case of Langmuir waves in a linear profile, or far from the apex of a parabolic profile, the steady state form of equation 5.3.1 can be written as

$$
\frac{\mathrm{d} \Psi_{\mathrm{sc}}}{\mathrm{dx}}=\frac{\omega_{\mathrm{pe}}^{2}\left(\hat{\mathrm{e}}_{\mathrm{pr}} \cdot \hat{\mathrm{e}}_{\mathrm{sc}}\right)}{4 \mathrm{ic}^{2} \mathrm{k}_{\mathrm{sc}}}\left(\Psi_{\mathrm{epw}} \Psi_{\mathrm{pr}}\right) \exp \left[\frac{\mathrm{i} \kappa^{\prime} \mathrm{x}^{2}}{2}\right]
$$


where the wavenumber mismatch, $\left(\vec{k}_{\mathrm{sc}}(\mathrm{x})-\overrightarrow{\mathrm{k}}_{\mathrm{pr}}(\mathrm{x}) \pm \overrightarrow{\mathrm{k}}_{\mathrm{epw}}(\mathrm{x})\right) \cdot \mathrm{d} \overrightarrow{\mathrm{x}} \approx \kappa^{\prime} \mathrm{x}$, has been Taylor expanded about the perfect phase matching point, $x=0$. Contour integration can be used to obtain the magnitude of the scattered vector potential as shown in Appendix 4.5. The resultant scattered vector potential, assuming a linear phase mismatch, is

$$
\left|\Psi_{\mathrm{sc}}\right|=\frac{\sqrt{2 \pi}}{\sqrt{\kappa^{\prime}} 4} \frac{\omega_{\mathrm{pe}}^{2}\left(\hat{\mathrm{e}}_{\mathrm{pr}} \cdot \hat{\mathrm{e}}_{\mathrm{sc}}\right)}{\mathrm{c}^{2} \mathrm{k}_{\mathrm{sc}}}\left|\Psi_{\mathrm{epw}}\right|\left|\Psi_{\mathrm{pr}}\right| .
$$

For Langmuir waves driven at the top of a parabolic profile, the steady state equation can be written in the form

$$
\frac{\mathrm{d} \Psi_{\mathrm{sc}}}{\mathrm{dx}}=\frac{\omega_{\mathrm{pe}}^{2}\left(\hat{\mathrm{e}}_{\mathrm{pr}} \cdot \hat{\mathrm{e}}_{\mathrm{sc}}\right)}{4 \mathrm{ic}^{2} \mathrm{k}_{\mathrm{sc}}}\left(\Psi_{\mathrm{epw}} \Psi_{\mathrm{pr}}\right) \exp \left[\frac{\mathrm{i} \kappa^{\prime} \mathrm{x}^{3}}{6}\right]
$$

where $\left(\vec{k}_{s c}(x)-\vec{k}_{p r}(x) \pm \vec{k}_{e p w}(x)\right) \cdot d \vec{x} \approx k^{\prime \prime} x^{2} / 2$ is the wavenumber mismatch at the top of a parabolic profile. Again contour integration can be used to obtain the magnitude of the scattered vector potential as shown in Appendix 4.5. The scattered vector potential at the top of a parabolic profile is

$$
\left|\Psi_{\mathrm{sc}}\right|=\left(\frac{6}{\kappa^{\prime \prime}}\right)^{1 / 3} \frac{\Gamma(1 / 3)}{\sqrt{3}} \frac{\omega_{\mathrm{pe}}^{2}\left(\hat{\mathrm{e}}_{\mathrm{pr}} \cdot \hat{\mathrm{e}}_{\mathrm{sc}}\right)}{4 \mathrm{c}^{2} \mathrm{k}_{\mathrm{sc}}}\left|\Psi_{\mathrm{epw}}\right|\left|\Psi_{\mathrm{pr}}\right|
$$

\subsection{Plasma Characterization}


The hydrodynamics code, LASNEX, was run by Kent Estabrook to model the plasmas used in this experiment. This modeling is important especially for thin foil targets used in the experiments described in this chapter. It is important that the peak of the laser intensity occur when the foil has burned through such that the maximum of the electron density is below the quarter critical surface where stimulated Raman scattering can be driven absolute on the top of the density profile. The LASNEX simulation used the incident laser power profile shown in figure 5.4.1 below. The maximum laser intensity occurred at $4.2 \mathrm{~ns}$ and the FWHM of the laser pulse was $2.5 \mathrm{~ns}$.

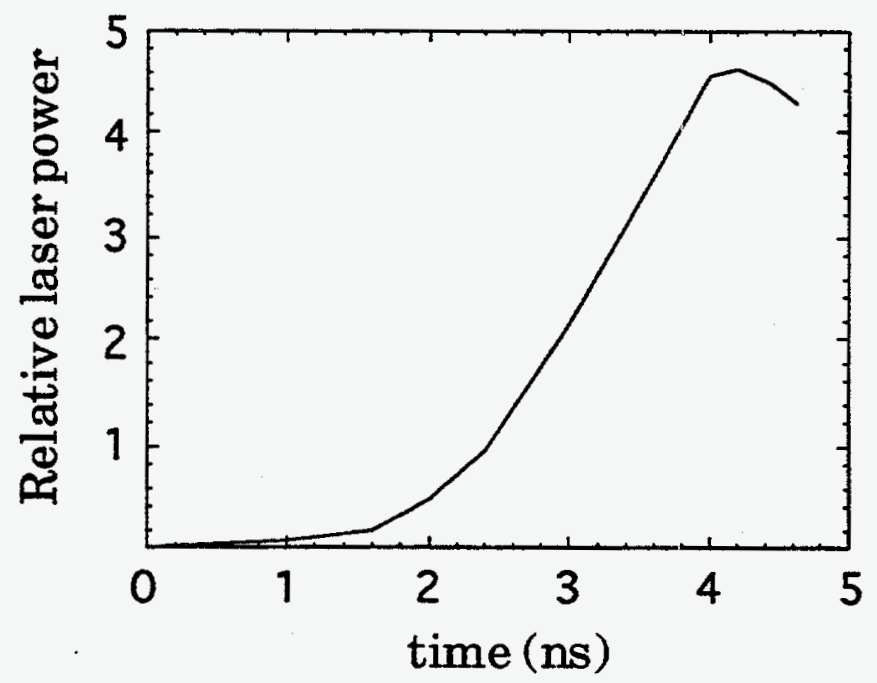

Figure 5.4.1 LASNEX model for the incident laser. The FWHM of the laser pulse is approximately $2.5 \mathrm{~ns}$ and the peak laser intensity occurs at $4.2 \mathrm{~ns}$.

Figure 5.4.2 shows the LASNEX prediction for the maximum electron density averaged over a $200 \mu \mathrm{m}$ radius as a function of time. This represents the case of a $0.40 \mu \mathrm{m}$ Collodium foil. In this simulation, by the time the laser reaches its maximum amplitude at 4.2 ns, the density has dropped to 
approximately $2.5 \%$ of the critical density. Most of the Raman signals seen in this experiment occurred below about $8 \%$ of the critical density for the 0.35 $\mu \mathrm{m}$ and below $5 \%$ of the critical density for $0.25 \mu \mathrm{m}$ targets. The scalelength, $L_{2}$, where $n_{e}=n_{o e} \exp \left[-\left(x / L_{2}\right)^{2}\right]$, is nearly $100 \mu m$ when the maximum density is approximately $6 \% \mathrm{n}_{\mathrm{cr}}$ to nearly $150 \mu \mathrm{m}$ when the maximum density has dropped to around $2 \% \mathrm{n}_{\mathrm{Cr}}$ at around $4.6 \mathrm{~ns}$ into the simulation.

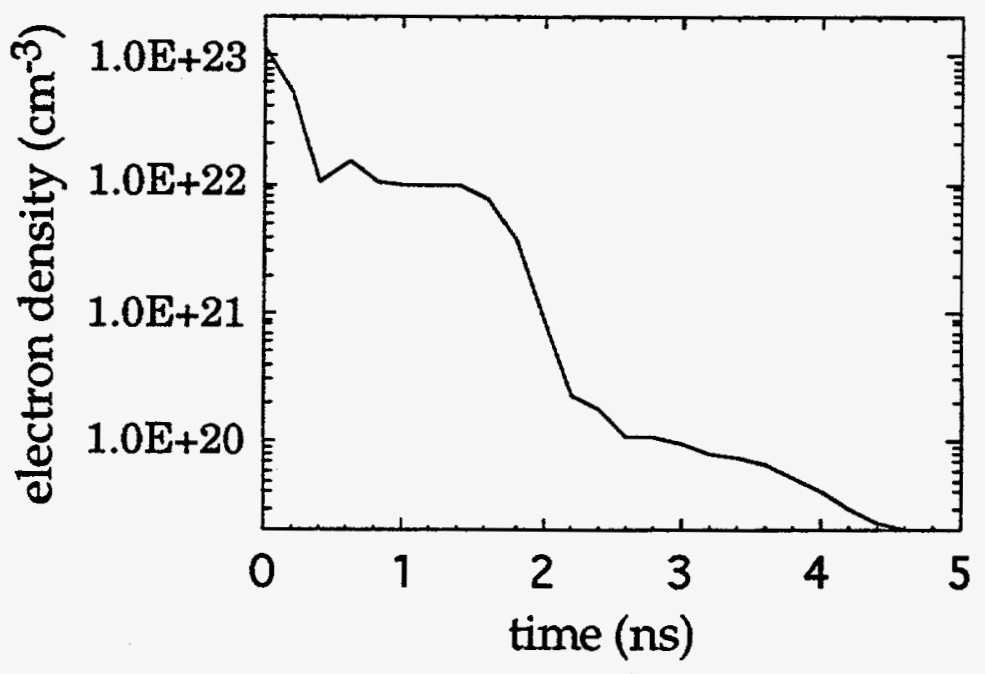

Figure 5.4.2 LASNEX prediction for the maximum electron density averaged over a $200 \mu \mathrm{m}$ radius cylinder as a function of time for a $0.40 \mu \mathrm{m}$ Collodium foil. The vertical axis shows the electron density while the horizontal axis represents time in ns.

Figure 5.4.3 shows the LASNEX prediction for the electron temperature as a function of time. The simulations placed the temperature of the plasma at approximately $350 \mathrm{eV}$ during the maximum of the laser intensity. The electron temperature peaks before the laser has reached the point of half the laser intensity, however, by this point the maximum density is dropping 
quickly. Because the laser absorption coefficient is quadratically dependent on the density, even though the laser intensity is increasing, less laser energy is being absorbed by the plasma and the electron temperature begins to cool even before the laser has reached half of its maximum intensity. Thomson scattering from thermal level ion acoustic waves was used in previous experiments to determine the electron temperature. These measurements estimated the temperature to be in the range $300-700 \mathrm{eV}$, consistent with the LASNEX results shown in figure 5.4.3[95]. The simulations also show that the plasma ions are fully stripped resulting in an average $\mathrm{Z}$ of approximately 5.29 .

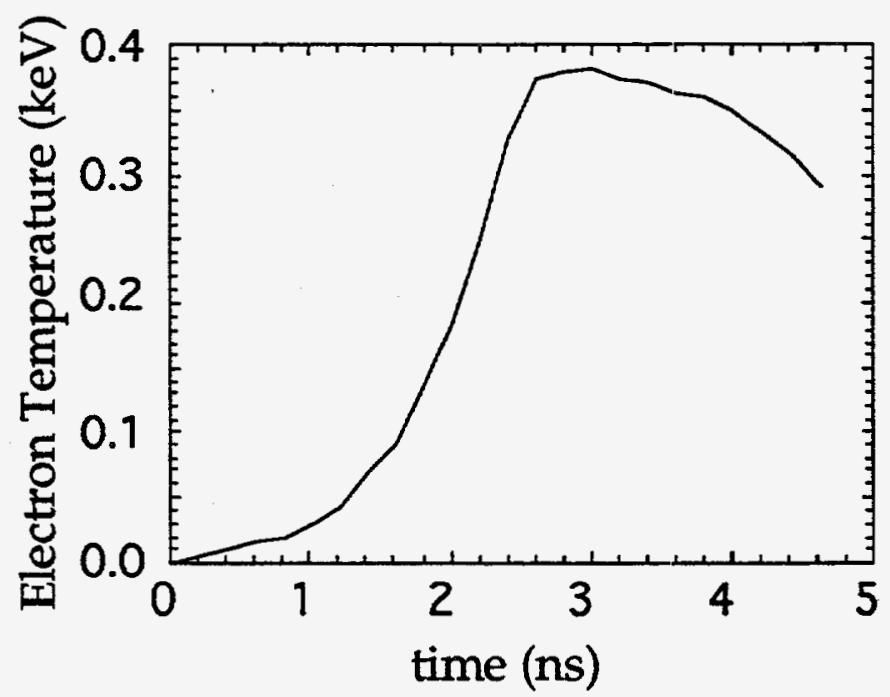

Figure 5.4.3 LASNEX estimation for the electron temperature averaged over a $200 \mu \mathrm{m}$ radius cylinder as a function of time for a $0.40 \mu \mathrm{m}$ Collodium foil target. The vertical axis shows the electron temperature while the horizontal axis represents time in ns.

The ion wave damping as a function of the ratio of ion temperature to electron temperature is shown in figure 5.4.4. The dispersion relation, 
$\varepsilon(\omega, \overrightarrow{\mathrm{k}})=1+\chi_{\mathrm{e}}(\omega, \overrightarrow{\mathrm{k}})+\sum_{\alpha} \chi_{\mathrm{i} \alpha}(\omega, \overrightarrow{\mathrm{k}})=0$, was solved to determine the damping on the ion acoustic waves present in the plasma. The dispersion relation was written in terms of complex error functions and solved using Mathematica. The simulations showed that the ratio of the ion temperature, $T_{i}$, to the electron temperature, $T_{e}$, was approximately 0.6 near the maximum of the electron density. According to figure 5.4.4, this ratio of temperatures would predict a damping of approximately $5 \%$ of the real component of the ion wave frequency.

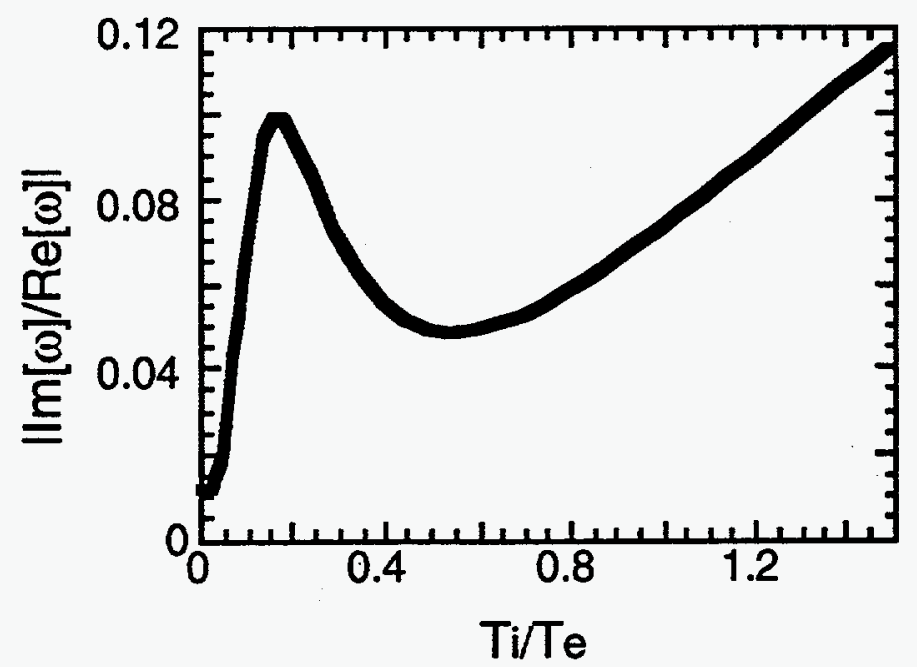

Figure 5.4.4 Ion wave damping as a function of the ratio of ion temperature, $T_{i}$, to electron temperature, $T_{e}$, for a Collodium plasma. The vertical shows the absolute magnitude of the ion wave damping normalized to the real part of the ion acoustic frequency and the horizontal axis represents the ratio of ion temperature, $T_{i}$, to electron temperature, $T_{e}$. This calculation assumed $k \lambda_{D e}=0.1$ and that the ions were completely ionized. 


\subsection{Experimental observations}

Figure 5.5.1 a shows the Thomson scattered measurement of the Langmuir wave spectrum obtained from a $0.35 \mu \mathrm{m}$ Collodium foil. The
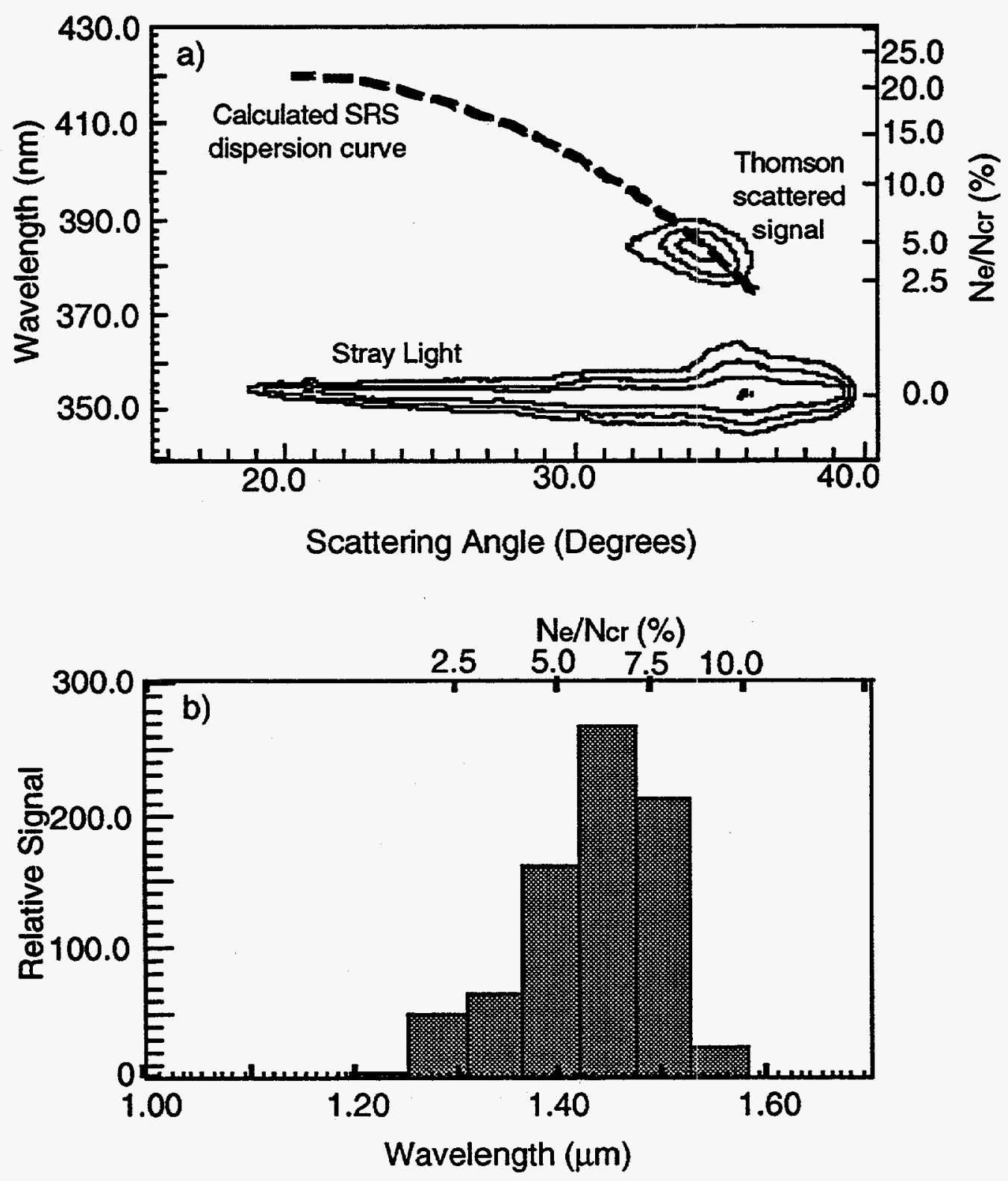
Figure 5.5.1 Thomson scattering and stimulated Raman scattering observed from a $0.35 \mu \mathrm{m}$ Collodium thin foil. In (a), the Thomson scattered signal is plotted along with the dispersion curve showing the expected position of the Thomson scattered signal as a function of wavelength. The contours in 5.5.1 a represent factors of two in intensity. In (b), the corresponding measurement of stimulated Raman scattering from the $1.064 \mu \mathrm{m}$ interaction beam is shown. The percentage of critical density from which the signals in (a) and (b) originated are drawn on each of the graphs for comparison.

feature near $355 \mathrm{~nm}$ represents stray probe light and Thomson scattering from ion acoustic waves. The dashed line represents the location which the Thomson scattered signal should lie assuming that the Langmuir waves are participating in resonant Raman scattering of the interaction beam. The feature from 380 to $390 \mathrm{~nm}$ represents Thomson scattering of the probe beam from Langmuir waves. This signal lies along the dashed curve expected for Langmuir waves participating in Raman scattering of the interaction beam. Figure 5.5.1 $\mathrm{b}$ represents the time-integrated measurement of the backscattered electromagnetic wave participating in stimulated Raman scattering. The inferred densities from which the Raman signal originated are drawn at the top of figure 5.5.1 b. This range of densities agrees with the inferred densities at which the Thomson scattered signal originated.

Figure 5.5.2 is a composite graph showing the Thomson scattering spectrum obtained from six different shots. These shots were taken from Collodium thin foil targets which ranged in thickness from $300 \mathrm{~nm}$ to 350 nm. In general, the Thomson scattered signal and the corresponding 
stimulated Raman signal resulting from the thicker targets originated from higher densities. The dashed line again represents the calculated curve corresponding to Langmuir waves participating in resonant scattering of the

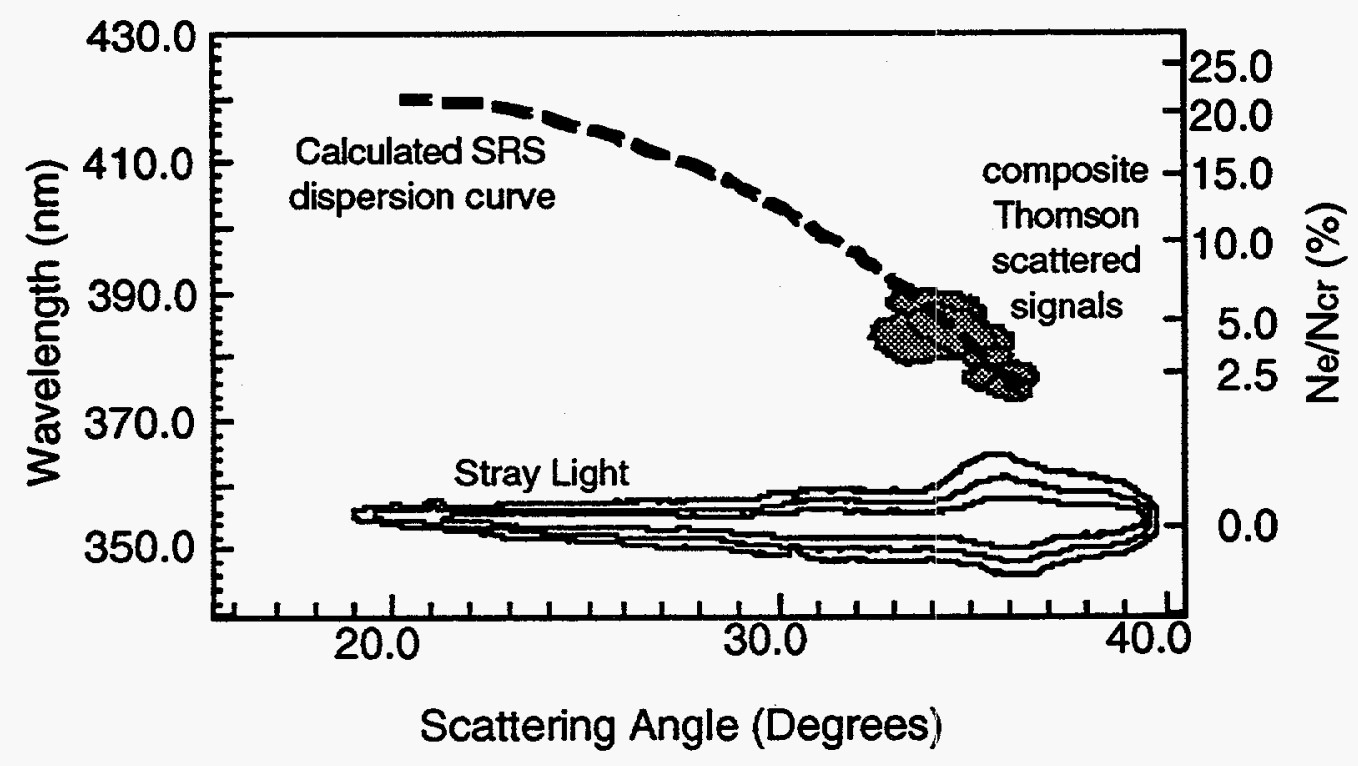

Figure 5.5.2 Composite graph showing the Thomson scattered signal from the Langmuir waves driven in six different shots. These signals all fall along the dispersion curve expected for resonant scattering of the interaction beam. The horizontal axis shows the angle which the light was scattered relative to the probe beam. The left vertical axis shows the wavelength of the scattered light and the right vertical axis shows the percent of critical density relative to the interaction beam which the signals originated, assuming an electron temperature of $350 \mathrm{eV}$.

incident pump beam. In all the cases in which Thomson scattering was observed, the Langmuir wave spectrum was found to lie along this dispersion 
curve. The filled contours represent the half intensity contour of the Thomson scattered signal for the respective shot.

The modulational instability, described in section 2.6, drives a range of ion wavenumbers unstable, causing a broadening in the Langmuir wave spectrum. For the experiments described in chapters 5 and 6 which saw a broadening in the Langmuir wave spectrum, the product of the wavenumber of the Langmuir waves and the Debye length was approximately $k_{e p w} \lambda_{D e} \approx 0.15$ to 0.3 . Figure 5.5 .3 below shows the range of unstable ion waves driven as a function of the amplitude of the pump Langmuir wave. The

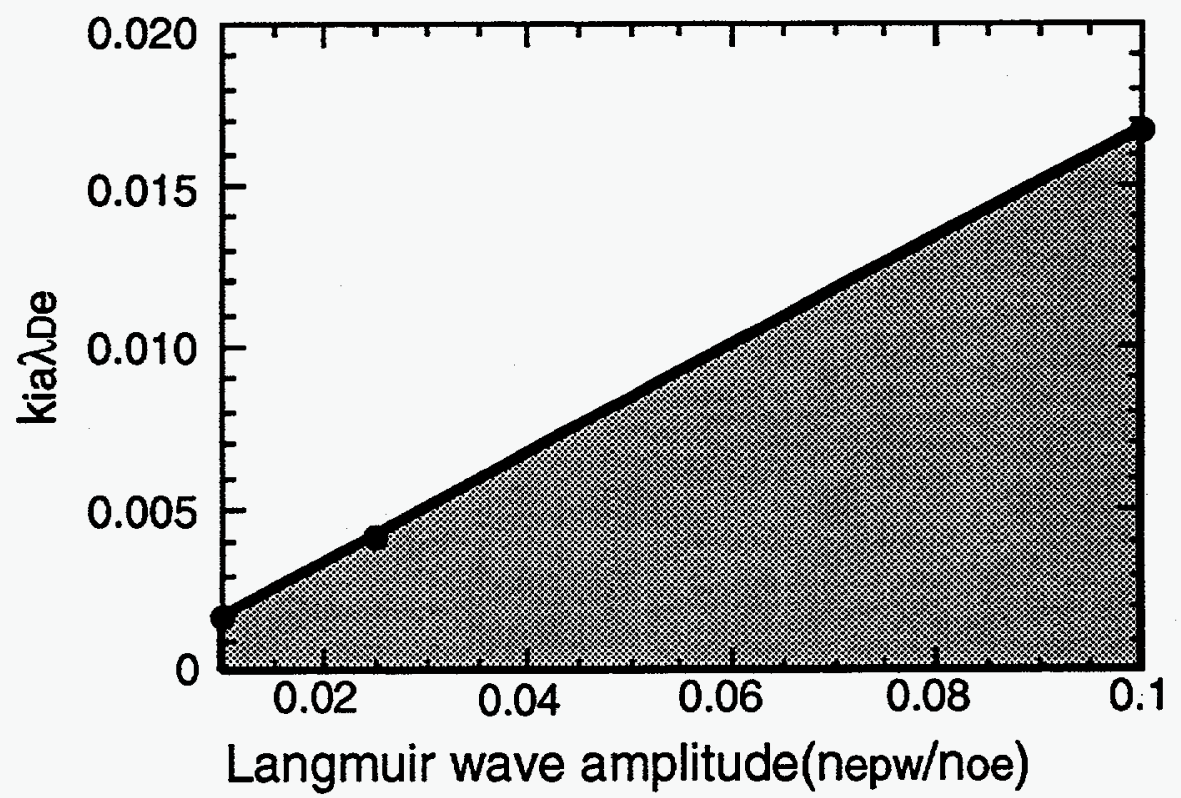

Figure 5.5.3 Range of unstable ion wavenumbers, $k_{i a}$, driven by the modulational instability. The horizontal axis represents the amplitude of the pump Langmuir wave while the vertical axis represents the wavenumber of the ion wave. The shaded region shows the unstable ion wavenumbers. This 
graph was made assuming that the phase velocity of the pump Langmuir was five times the thermal velocity of the plasma or $k_{e p w} \lambda_{D e}=0.2$.

broadest signal observed represented an $8 \%$ change in wavenumber across the angular width of the signal with the majority of the shots having an angular width corresponding to only a $2 \%$ change in wavenumber. For $k_{e p w} \lambda_{D e} \approx 0.2$, the modulational instability would predict a Langmuir wave amplitude of approximately $n_{\text {epw }} / n_{0 e} \approx 9.5 \%$ for the broadest case, $8 \%$, and an amplitude of approximately $n_{\text {epw }} / n_{0 e} \approx 2.4 \%$ for the average case, $2 \%$. The broadest signal shown in composite graph showed a weaker Thomson scattering and Stimulated Raman scattering signal than observed in figure 5.5.1 above indicating that the broadening of the Langmuir wave spectrum is not correlated with the Langmuir wave amplitude as measured by the intensity of the Thomson scattering spectrum.

\subsection{Discussion}

The broadest signal shown in figure 5.5 .2 represents less than an 8 percent change in the Langmuir wavevector, which is much narrower than the spectrum of Langmuir waves expected from Langmuir waves driven due to the bump-on-tail instability. In addition, the largest percentage of shots showed less than a 2 percent change in the magnitude of the Langmuir wavenumber. It is unlikely that the spectrum of Langmuir waves driven by the bump-on-tail instability could be this narrow. The bump-on-tail instability should drive a very broad spectrum of Langmuir waves, only a 
small fraction of which would be resonant for Raman scattering[96]. That is not to say that enhanced Langmuir waves were not present, but that the amplitude of such Langmuir waves would be down in magnitude by at least a factor of 20 from the stimulated-Raman-scattering-driven Langmuir waves detected in this experiment. The bump-on-tail instability can also drive quasiLangmuir waves which do not satisfy the normal dispersion relation for a Langmuir wave in a plasma with a monotonically decreasing distribution function. These quasi-Langmuir waves which do not obey the normal dispersion relation cannot decay easily through wave-wave interaction. These waves would likely be driven to a larger amplitude than their normal mode counterparts, producing a larger Thomson scattering signal. These quasimodes were not observed in this experiment.

The enhanced Thomson scattering model requires a source of hot electrons from which the Langmuir waves are driven. The source most frequently assumed is the two plasmon decay instability near the quarter critical surface. In the case of the majority of the targets used in this experiment, the center of the foil burned through the quarter critical surface before the end of the incident laser pulse, although there remained a quarter critical surface near the edges of the interaction beam. For the $0.4 \mu \mathrm{m}$ thick targets, predicted that the foils went below quarter critical approximately $\mathbf{2 . 2}$ ns into the laser pulse. A more thorough test of the enhanced Thomson scattering model would involve using targets which retained their quarter critical surface the entire time the laser is interacting with the plasma. With the given combination of laser energy and focusing optic, it was not possible to drive stimulated Raman scattering from a solid Carbon target even using a 
5 ns prepulse in an attempt to increase the scalelength. It was possible, however, to drive two plasmon decay from these targets which was inferred from the $3 / 2 \omega_{0}$ emission and $5 / 2 \omega_{0}$ emission measured from the solid targets. Figure 5.6.1 shows a time integrated $3 / 2 \omega_{0}$ spectrum which resulted from a solid Carbon target. The $3 / 2 \omega_{0}$ light is produced when the incident laser Thomson scatters from Langmuir waves produced near the quarter critical surface[76]. Therefore, although it was not possible to observe Raman signals from these solid targets, it was inferred that two plasmon decay was occurring. Therefore, the lack of Raman in the solid target experiments indicated that the Langmuir waves were not driven sufficiently by the bumpon-tail instability to be observed with the Au:Ge detector.

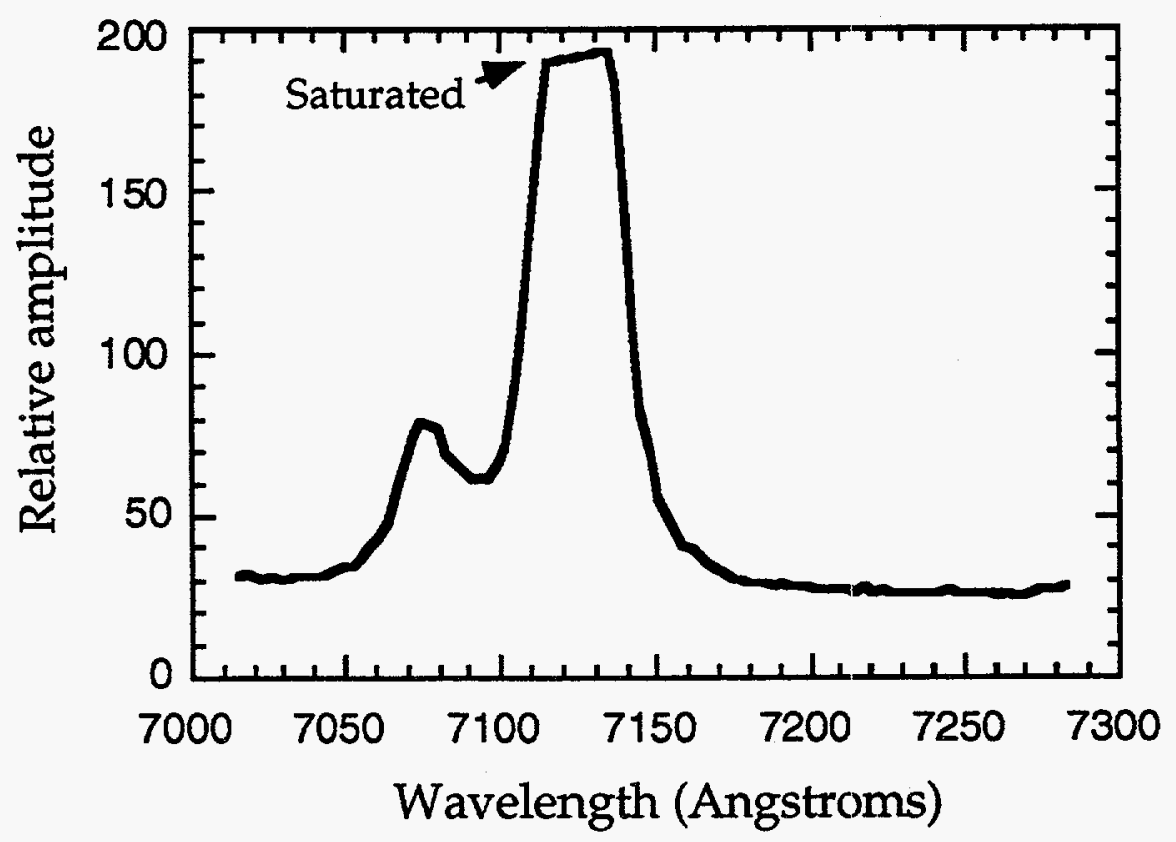

Figure 5.6.1 The 3/2 $\omega_{0}$ emission obtained from a solid Carbon target which maintained a critical surface throughout the duration of the interaction beam. The vertical axis shows the relative amplitude to which the peaks were 
driven, while the horizontal axis represents the wavelength of the scattered light.

The Thomson scattered vector potential resulting from a parabolic profile can be much larger than the vector potential resulting from a linear profile, assuming comparable scalelengths and Langmuir wave amplitudes. It is therefore probable that the Raman signal produced by the enhanced Thomson scattering model would be much larger in the case of a parabolic profile than in the case of a linear profile. The ratio of the scattered vector potentials produced in a linear profile, $\left|\Psi_{\mathrm{sc}}\right|_{\text {lin }}$, to that produced in a parabolic profile, $\left|\Psi_{\text {sc }}\right|_{\text {par }}$ is $\left|\Psi_{\text {sclin }} /\right| \Psi_{\text {sclpar }} \approx 1.5\left(\mathrm{k} \lambda_{\text {De }}^{2}\right)^{1 / 6} \sqrt{L_{\text {lin }}} /\left(L_{\text {par }}\right)^{2 / 3}$, where Llin and $L_{\text {par }}$ are the scalelengths for the linear and parabolic profiles respectively. For the parameters present in this experiment, the vector potential produced from the linear profiles present in the solid Carbon targets should have been about one fourth of the amplitude of the vector potential produced in the Collodium foil targets. This signal level should still have been measurable with the backscatter diagnostic, provided the Langmuir waves had been driven to the same amplitude by the enhanced Thomson scattering model.

\subsection{Summary}

In summary, Thomson scattering was used to measure the Langmuir wave spectrum associated with Raman backscatter. This measurement showed that the Langmuir waves obeyed the dispersion relation for Langmuir waves driven by stimulated Raman scattering. The Thomson 
scattered signal was observed over a narrow angular range which is consistent with the assumption that the Langmuir waves were driven by stimulated Raman scattering and inconsistent with the assumption that they were driven by the bump-on-tail instability predicted in the enhanced Thomson scattering model. In the solid Carbon target experiments, two plasmon decay was driven, as inferred from measurements of $3 / 2 \omega_{0}$ emission. Stimulated Raman scattering, however, was not detected in these experiments which retained a quarter-critical surface for the entire duration of the interaction beam. The lack of stimulated Raman scattering was expected due to the relatively steep density gradient[97]. The enhanced Thomson scattering model, however, would predict the observation of signals in this case due to the presence of two plasmon decay. Therefore, the lack of Raman signals in the solid target experiments shows that the Langmuir waves were not driven sufficiently by the bump-on-tail instability. 


\section{Chapter 6}

\section{Thomson scattering measurements of the Langmuir wave spectra resulting from stimulated Raman scattering}

\subsection{Introduction}

This chapter shows the results of an experiment in which Thomson scattering was used to measure the Langmuir wave spectrum generated by stimulated Raman scattering. This experiment detected Langmuir waves with components both parallel and antiparallel to the incident laser's wavevector, $\overrightarrow{\mathrm{k}}_{\mathrm{o}}$. The parallel component was attributed to stimulated Raman scattering. However, the Langmuir waves moving antiparallel to $\vec{k}_{0}$, which cannot be explained by stimulated Raman scattering, were attributed to the Langmuir decay instability.

As discussed in chapter 1, saturation of the amplitude of Langmuir waves is an important issue in many applications involving plasmas. These 
applications include inertial confinement fusion[98], particle accelerators[2], current-drive in Tokamaks[5], and X-ray lasers[1]. In laser-produced plasmas, Langmuir waves may be driven by several instabilities, one of which is stimulated Raman scattering(SRS). In SRS, an incident electromagnetic wave resonantly drives a Langmuir wave and a scattered electromagnetic wave[16,17]. Much of the prior work on SRS has been focused on its onset, which proved difficult to understand[67,99-102]. Few experiments have looked directly for these saturation mechanism. $s[3,40,56]$. Many recent simulations and analyses have indicated that the Langmuir decay instability may be responsible for the saturation of $\operatorname{SRS}[9,12,13,39]$. The present work is the first experimental study of the three wave process known as the Langmuir decay instability in laser-produced plasmas.

The Langmuir decay instability involves the decay of a Langmuir wave into a second Langmuir wave and an ion acoustic wave. This instability can be driven by the Langmuir waves produced by stimulated Raman scattering, two plasmon decay, the ion acoustic decay instability, and other mechanisms, as well. For the present experiment stimulated Raman scattering was chosen to provide the Langmuir pump wave. The electromagnetic daughter wave associated with stimulated Raman scattering is easily diagnosed for a direct comparison to the measured Langmuir waves. The primary Langmuir wave spectrum driven by stimulated Raman scattering is also much simpler than the primary spectrum driven by either the ion acoustic decay instability or two plasmon decay. Specifically, two plasmon decay and the ion acoustic decay instability generate Langmuir waves which have components both parallel and antiparallel to the incident laser wavevector. In contrast, 
stimulated Raman scattering only drives Langmuir waves with components in the same direction as the incident laser wavevector. Thus, any Langmuir waves traveling antiparallel to the laser wavevector must be due to the Langmuir decay instability or another mechanism which can reverse the wavevector of the Langmuir wave.

\subsection{Experimental layout}

The layout of the experiment is shown in figure 6.2.1. The plasma was formed by two counterpropagating $0.526 \mu \mathrm{m}$ beams impinging upon a $1.2 \mu \mathrm{m}$ thick, $440 \mu \mathrm{m}$ diameter, $\mathrm{CH}$ foil. These $\mathrm{f} / 6$ plasma formation beams contained Random Phase Plates with $0.75 \mathrm{~mm}$ elements which produced an approximate FWHM focal spot size on target of $360 \mu \mathrm{m}$. The average intensity on target for the preform beams was approximately $5 \times 10^{13} \mathrm{~W} / \mathrm{cm}^{2}$. An $\mathrm{f} / 6$ heater beam at $0.526 \mu \mathrm{m}$, delayed by $1.1 \mathrm{~ns}$ from the preform beams, provided a measurement of the temporal evolution of the plasma density, as well as a temperature diagnostic. The heater beam contained a Random Phase Plate with $1.5 \mathrm{~mm}$ elements which produced an approximate FWHM focal spot size of $180 \mu \mathrm{m}$. The average intensity on target for the heater beam was approximately $2 \times 10^{14} \mathrm{~W} / \mathrm{cm}^{2}$. The interaction beam at $1.053 \mu \mathrm{m}$ was incident upon the plasma approximately $1.7 \mathrm{~ns}$ after the plasma formation beams. The $\mathrm{f} / 6$ interaction beam had a temporal length of approximately $600 \mathrm{ps}$. Without a Random Phase Plate, the average intensity on target for the interaction beam was approximately $3 \times 10^{14} \mathrm{~W} / \mathrm{cm}^{2}$. When a Random Phase Plate was used on the interaction beam, its element size was $2 \mathrm{~mm}$ producing an 
approximate FWHM focal spot size of $260 \mu \mathrm{m}$. This resulted in a lower intensity on target of approximately $5 \times 10^{13} \mathrm{~W} / \mathrm{cm}^{2}$.

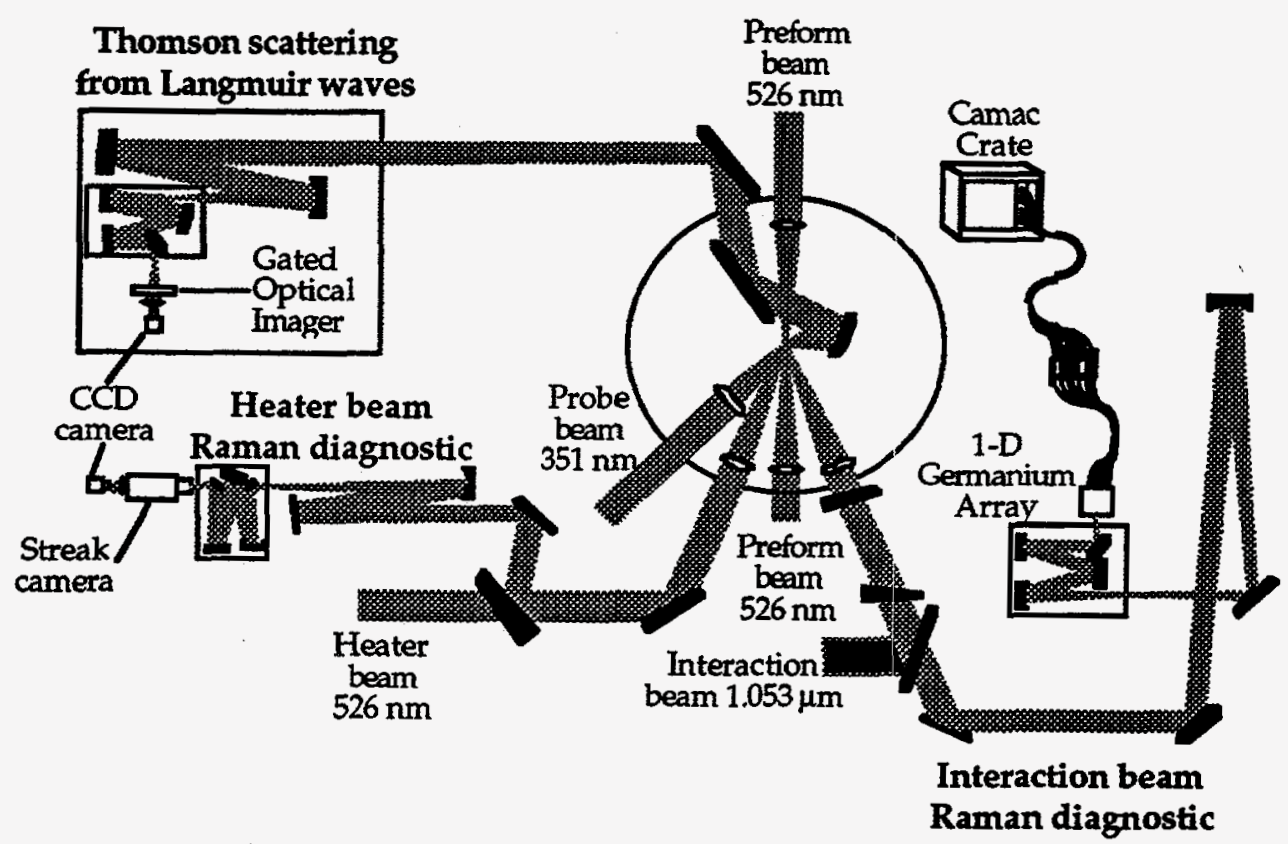

Figure 6.2.1 Experimental setup showing the Thomson scattering diagnostic, as well as the stimulated Raman scattering diagnostics from the $526 \mathrm{~nm}$ heater beam and the $1.053 \mu \mathrm{m}$ interaction beam.

The Thomson scattering probe beam at $351 \mathrm{~nm}$ was incident on the plasma approximately $1.4 \mathrm{~ns}$ after the plasma formation beams. The use of a 3 to 1 ratio for the probe beam frequency to interaction beam frequency allowed the Thomson downscattered signal from the Langmuir waves traveling in the same direction as the laser wavevector to be scattered at nearly the same angle as the Thomson upscattered signal from the Langmuir waves traveling in the opposite direction to the laser wavevector. The Thomson scattered beam had an $f / \#$ of 3.33 which in some shots was changed to an $f / \#$ of 25 
through the use of an aperture. The angular spread of the Thomson probe beams and interaction beams are important in analyzing the angular extent of the Thomson scattered signals as discussed below. With an $f / \#$ of 3.33 the probe beam had an angular spread of approximately 17 degrees, however, with the use of the aperture, the angular spread was reduced to approximately 2.3 degrees. The interaction beam had an f/\# of 6 which resulted in an angular spread of approximately 9.5 degrees.

The stimulated Raman backscatter from the $1.053 \mu \mathrm{m}$ interaction beam was monitored to provide a comparison with the Thomson scattered signal resulting from the driven Langmuir wave spectrum. The wavelength range corresponding to stimulated Raman scatter from the interaction beam ranges from $2 \mu \mathrm{m}$ near the quarter critical surface to approximately $1 \mu \mathrm{m}$ at very low densities. The time integrated stimulated Raman backscatter from the $1 \mu \mathrm{m}$ interaction beam was recorded using a room-temperature 1-D Germanium photodiode array, which was sensitive from $800 \mathrm{~nm}$ to approximately $1.8 \mu \mathrm{m}$. The Germanium photodiode array consisted of 16 elements, each element having a width of $1 \mathrm{~mm}$. This array was placed at the output plane of a 0.35 meter Czurney-Turner spectrometer. Each of the channels in the array was biased positive to 3.2 volts to improve linearity and response time(lower the junction capacitance). The diagram of the detector circuit is shown in figure 6.2.2. The output of the diode was sent through a high-pass filter and read out using LeCroy 2249A charge integrators. The Raman backscatter spectrometer had a $100 \mathrm{gr} / \mathrm{mm}$ grating which yielded a dispersion of approximately 30.3 $\mathrm{nm} / \mathrm{mm}$. Therefore, each channel of the detector sampled $30.3 \mathrm{~nm}$. An example of the Raman spectrum can be seen in figure $6.4 .1 \mathrm{~b}$. The output 
signal from the 2249A charge integrator was then read using LabView on the Macintosh.

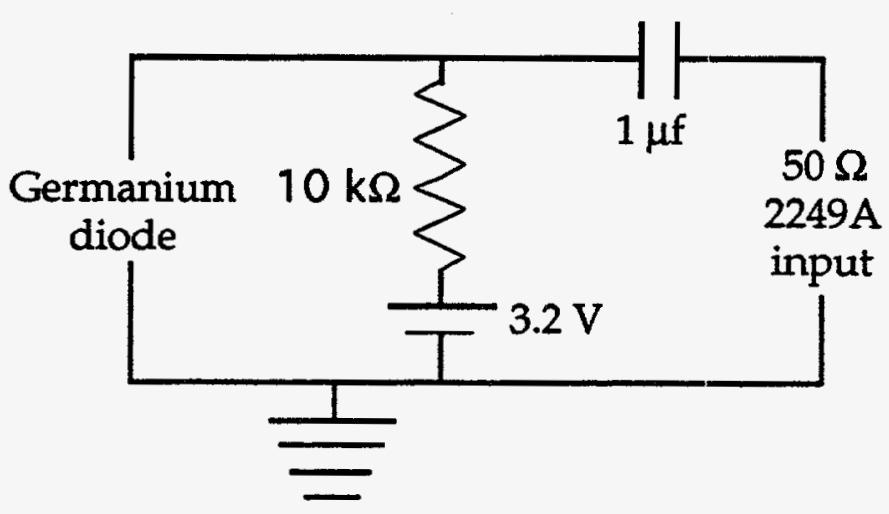

Figure 6.2.2 Schematic of one of the detector circuits used for the Germanium diode array. The $50 \Omega$ input from the oscilloscope, in conjunction with the 0.6 nf junction capacitance of the Germanium diodes, gave an RC decay time of approximately $33 \mathrm{~ns}$.

Figure 6.2.3 shows a trace of one of the channels of the diode array, as well as the gate signal for the 2249A. The 2249A collected charge from the diode array during the $200 \mathrm{~ns}$ gate signal. The junction capacitance of the diodes was reduced to approximately $0.6 \mathrm{nf}$ when the diode was biased to 3.2 volts. This gave the detector circuit a RC decay time of approximately $33 \mathrm{~ns}$ which was sufficiently short that the signal could be integrated over the 200 ns gate time.

The backscattered stimulated Raman scattering from the heater beam provided information on the peak plasma density as a function of time. This signal ranged from $1053 \mathrm{~nm}$ at the quarter critical surface to around $526 \mathrm{~nm}$ at very low densities. This diagnostic could also measure the $3 / 2 \omega_{0}$ emission, at $702 \mathrm{~nm}$, generated from the $1053 \mathrm{~nm}$ interaction beam, as well as Thomson 
upscatter from the interaction beam. The Raman backscattered signal was dispersed using a $0.25 \mathrm{~m}$ Czurney-Turner spectrometer and temporally

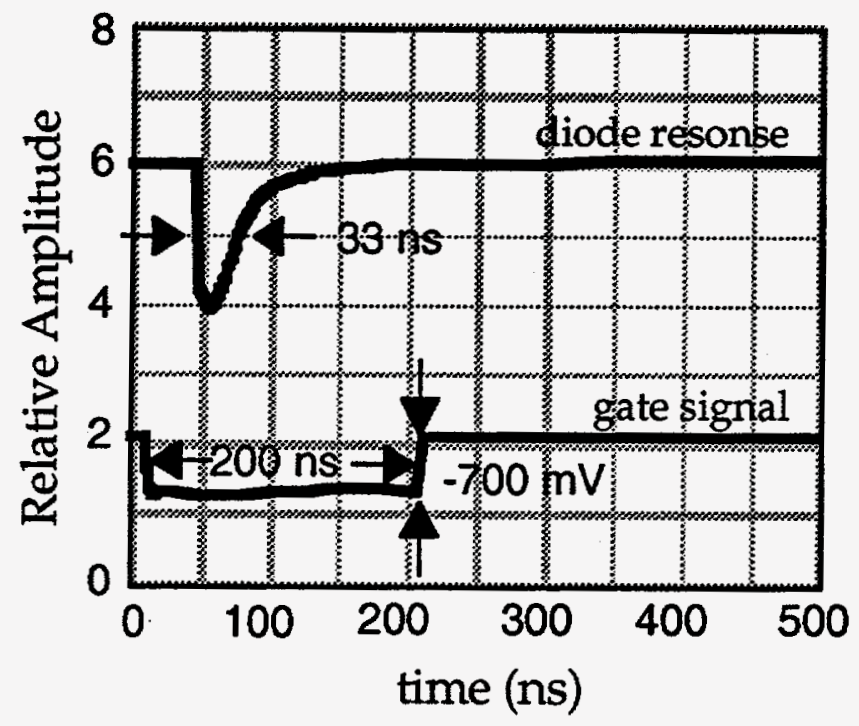

Figure 6.2.3 Oscilloscope trace showing the gate signal, lower trace, which was sent to the 2249 A charge integrator and the diode response, higher trace, to a $600 \mathrm{ps} 1 \mu \mathrm{m}$ laser impinging upon its surface. The $2249 \mathrm{~A}$ integrated the current from the diode over the $200 \mathrm{~ns},-700 \mathrm{mV}$ gate signal.

dispersed by a S-20 Livermore streak camera placed at the output plane of the spectrometer. The streak camera had the $3 X$ card which provided a temporal window of approximately $3 \mathrm{~ns}$. By carefully choosing the filtering to allow through a small amount of $526 \mathrm{~nm}$ light, the streak camera provided information on the relative timing of the beams. This technique also allowed for the wavelength calibration on each shot. The first order signal from the grating provided wavelength for $526 \mathrm{~nm}$ and the second order signal from the grating provided the location of the $1053 \mathrm{~nm}$ wavelength. The output 
phosphor of the streak camera was imaged onto a Photometrics CCD camera which recorded each shot. The Stimulated Raman signal was also sent to a photodiode for absolute measurement of the backscattered signal.

The Thomson scattered signals from the Langmuir waves and the ion acoustic waves were relayed out of the chamber using the same optical path. A beamsplitter was used to send the Thomson scattered signals to two different optical tables. One which observed Thomson scattering from the Langmuir waves and the other which measured Thomson scattering from the ion acoustic waves. The Langmuir wave optical table, shown in figure 6.2.1, consisted of an S-20 gated optical imager, and a $0.45 \mathrm{~m}$ Czurney-Turner spectrometer. The grating used in the spectrometer was a $300 \mathrm{gr} / \mathrm{mm}$ grating which resulted in a dispersion of $10 \mathrm{~nm} / \mathrm{mm}$ at the output plane of the spectrometer. As discussed in chapter 5, the Fourier transform plane of the Thomson scattered signal from the Langmuir waves was focused onto the entrance slit of the spectrometer which allowed for the angular measure in one direction and wavelength resolution in the perpendicular direction[40,103]. The S-20 gated optical imager was placed at the output of the spectrometer to provide a time integrated measurement of $\omega \mathrm{vs}$. $k$ of the Langmuir waves present in the plasma. The output phosphor of the gated optical imager was recorded with a CCD camera.

The ion acoustic optical table consisted of a $1.2 \mathrm{~m}$ Ebert-Fastie spectrometer and an S-20 Imacon 500 streak camera. The image plane of the plasma was focused onto the entrance slit of the spectrometer. The spectrometer had a $1200 \mathrm{gr} / \mathrm{mm}$ grating which produced a $0.7 \mathrm{~nm} / \mathrm{mm}$ dispersion at its output. A spherical lens was used to image the output of the 
spectrometer onto the entrance slit of the streak camera for frequency vs. time measurements. The output phosphor of the streak camera was then recorded on hard film.

\subsection{Plasma Characterization}

The hydrodynamics code, LASNEX, was run by Kent Estabrook to model the $\mathrm{CH}$ plasmas used in this experiment. This modeling was essential in determining the proper target thickness and beam timing such that the correct range of maximum electron densities would overlap temporally with the interaction beam. The LASNEX run described below assumed that the plasma was formed by two $0.527 \mu \mathrm{m}$ preform beams at time $t=0$ which were $640 \mathrm{ps}$ in duration and contained 26 Joules of energy. The heater beam in the simulation was 640 ps in duration and contained 26 Joules of energy. The heater beam was modeled as a $0.527 \mu \mathrm{m}$ beam which was incident $1 \mathrm{~ns}$ after the preform beams. Both the preform beams and the heater beam were simulated as $f / 6$ beams. The interaction beam was also $640 \mathrm{ps}$ in temporal width and was modeled as an $\mathrm{f} / 12$ beam. The interaction beam was a $1.05 \mu \mathrm{m}$ beam which was delayed 2 ns from the preform beams. The FWHM of the spot size on target of the beams in the simulation were $393 \mu \mathrm{m}, 450 \mu \mathrm{m}$, and $250 \mu \mathrm{m}$ for the preform beams, the heater beam, and the interaction beam, respectively. The on target intensities in the simulations were $2.5 \times 10^{13}$ $\mathrm{W} / \mathrm{cm}^{2}, 1.9 \times 10^{13} \mathrm{~W} / \mathrm{cm}^{2}$, and $1.2 \times 10^{14} \mathrm{~W} / \mathrm{cm}^{2}$ for the preform beams, the heater beam, and the interaction beam, respectively. The $\mathrm{CH}$ target was 1.2 
$\mu \mathrm{m}$ thick and approximately $400 \mu \mathrm{m}$ in diameter. The flux limit used in the simulations was 0.03 .

The LASNEX prediction for the maximum density as a function of time near the center of the target is shown below in figure 6.3.1. In this figure the beam timing is drawn in at the bottom of the graph showing the preform beams at $t=0 \mathrm{~ns}$, the heater beam at $\mathrm{t}=1 \mathrm{~ns}$, and the interaction beam at $\mathrm{t}=2 \mathrm{~ns}$. The solid line represents the LASNEX prediction for the density evolution in time, while the gray striped line shows the density versus time inferred from Raman scattering measurements taken during the experiment. The targets used in these experiments are similar to those used in previous

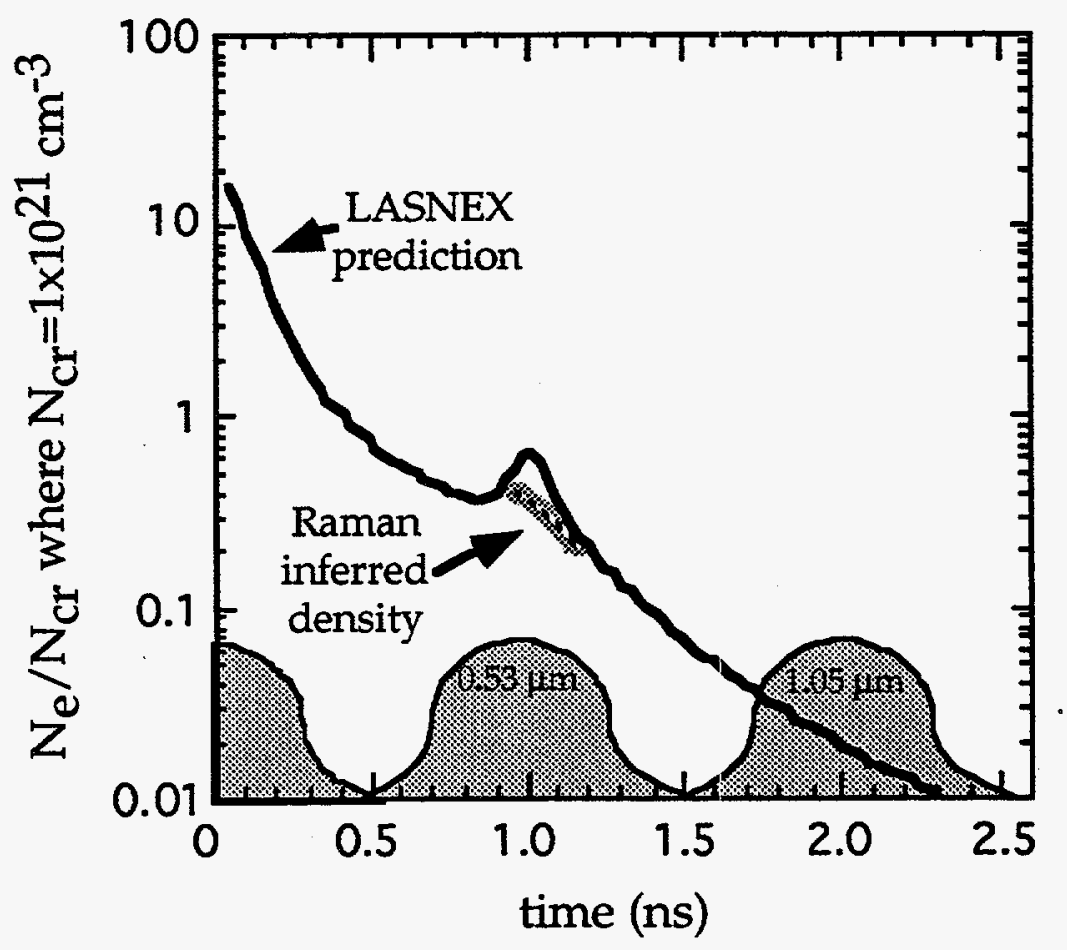

Figure 6.3.1 LASNEX predicted evolution of the maximum of the plasma density as a function of time. The vertical axis shows the electron density normalized to the critical density for a $1 \mu \mathrm{m}$ interaction beam. The horizontal 
axis represents the time in the simulation. The beam timing used in the simulation is drawn at the bottom of the figure.

experiments[104]. A brief density increase in time was also seen in the simulations described in the reference above and interpreted as an inflow of plasma from the edges of the target stagnating at the center of the target. This interpretation is consistent with the reaction of the ion temperature in these simulations as discussed below. Over the density range where the maximum density falls from approximately 16 percent of the critical density for the interaction beam to approximately 1 percent of the critical density, the LASNEX prediction for the density evolution as a function of time can be fit by the relation $n_{e} / n_{C r} \approx 0.415(t)^{-4.43}$.

The LASNEX prediction for the evolution of the plasma temperature is shown in figure 6.3.2 below. The electron temperature follows closely the time history of the incident beams. The collisional damping of the incident beams due to inverse bremsstrahlung strongly affects the temperature of the electrons as can be seen in figure 6.3.2. The ion temperature experiences a large spike near $1 \mathrm{~ns}$ and becomes larger than the electron temperature in the simulation. This occurs at the same time as the appearance of the density increase in time at the center of the target shown in figure 6.3.1 above. The interpretation of the density hump as being produced by plasma driven from the edges is consistent with the jump in ion temperature. As the plasma begins to interpenetrate at the center of the plasma, the plasma stagnates causing a large heating of the ions which might or might not be realistic[105]. 


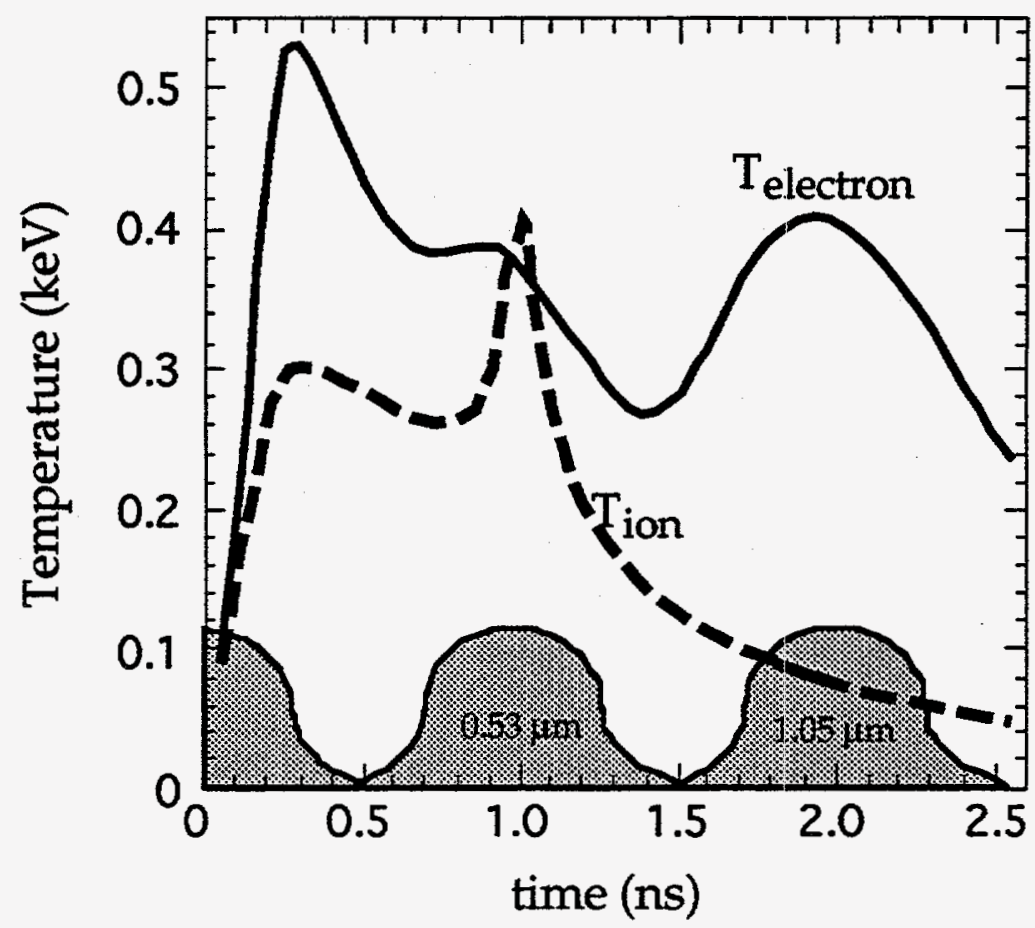

Figure 6.3.2 LASNEX prediction for time evolution of the electron and ion temperatures present in the plasma. This represents the temperatures at the center of the targets. The vertical axis is temperature while the horizontal axis is the time of the simulation. Again the beam timing used in the simulation is drawn at the bottom of the figure.

The LASNEX prediction for the linear flow velocity scalelength at the time of the interaction beam was approximately $400 \mu \mathrm{m}$ out to about $\mathrm{z}=500$ $\mu \mathrm{m}$ beyond which the scalelength goes to approximately $700 \mu \mathrm{m}$. The spatial density profile is not well characterized by a gaussian density profile. However, the density scalelength is very large being on the order of $700 \mu \mathrm{m}$.

The maximum density of the plasma was determined from measurements of the stimulated Raman scattering from the heater beam, as shown in figure 6.3.3. The beam timing as measured by this diagnostic is 
drawn to the right of the figure. The features near $t=0$ are the two preform beams at $526 \mathrm{~nm}$ appearing on the first and second order of the grating. The interaction beam drives two plasmon decay when densities higher than approximately twenty percent of the critical density for the interaction beam are present[76]. The feature at $702 \mathrm{~nm}$, driven from $t=1.2$ to $t=1.6 \mathrm{~ns}$, represents the Thomson scattering of the interaction beam from the Langmuir waves associated with two plasmon decay as discussed in chapter 3 . The feature above $750 \mathrm{~nm}$, driven from $\mathrm{t}=1.45$ to $\mathrm{t}=1.65 \mathrm{~ns}$, represents Thomson upscattering of the interaction beam from non-thermal-level Langmuir waves. The feature above $700 \mathrm{~nm}$, driven from $t=0.8$ to $t=1.25 \mathrm{~ns}$, represents stimulated Raman scattering which is driven when the heater beam is turned on. The density inferred from the measurement of the maximum of the stimulated Raman scattering spectrum, from 0.9 to $1.2 \mathrm{~ns}$, as a function of time is drawn on figure 6.3.1, which shows the LASNEX prediction for the density evolution as a function of time. Although the density deduced from the Raman scattering does not follow the brief temporal density increase shown in the simulation, it does agree with the temporal slope of the LASNEX density profile after the brief temporal density increase.

This diagnostic can also be used as a rough estimate of the electron temperature of the plasma[95,99]. The idea behind this temperature diagnostic is the rapid increase in the Landau damping of the Langmuir wave as a function of the parameter $k \lambda_{D e}$, the product of the Langmuir wave's wavenumber with the Debye length. The short wavelength limit of the scattered spectrum would then be interpreted as being the result of the increased Landau damping on the Langmuir wave. As shown in figure 2.10.1 
from chapter 2, the shorter wavelengths of Raman scattered light correspond to larger values of $k \lambda_{\mathrm{De}}$. For absolute instability, the homogeneous damping threshold occurs when the homogeneous growth rate, $\gamma_{\text {OSRS, }}$ is equal to one

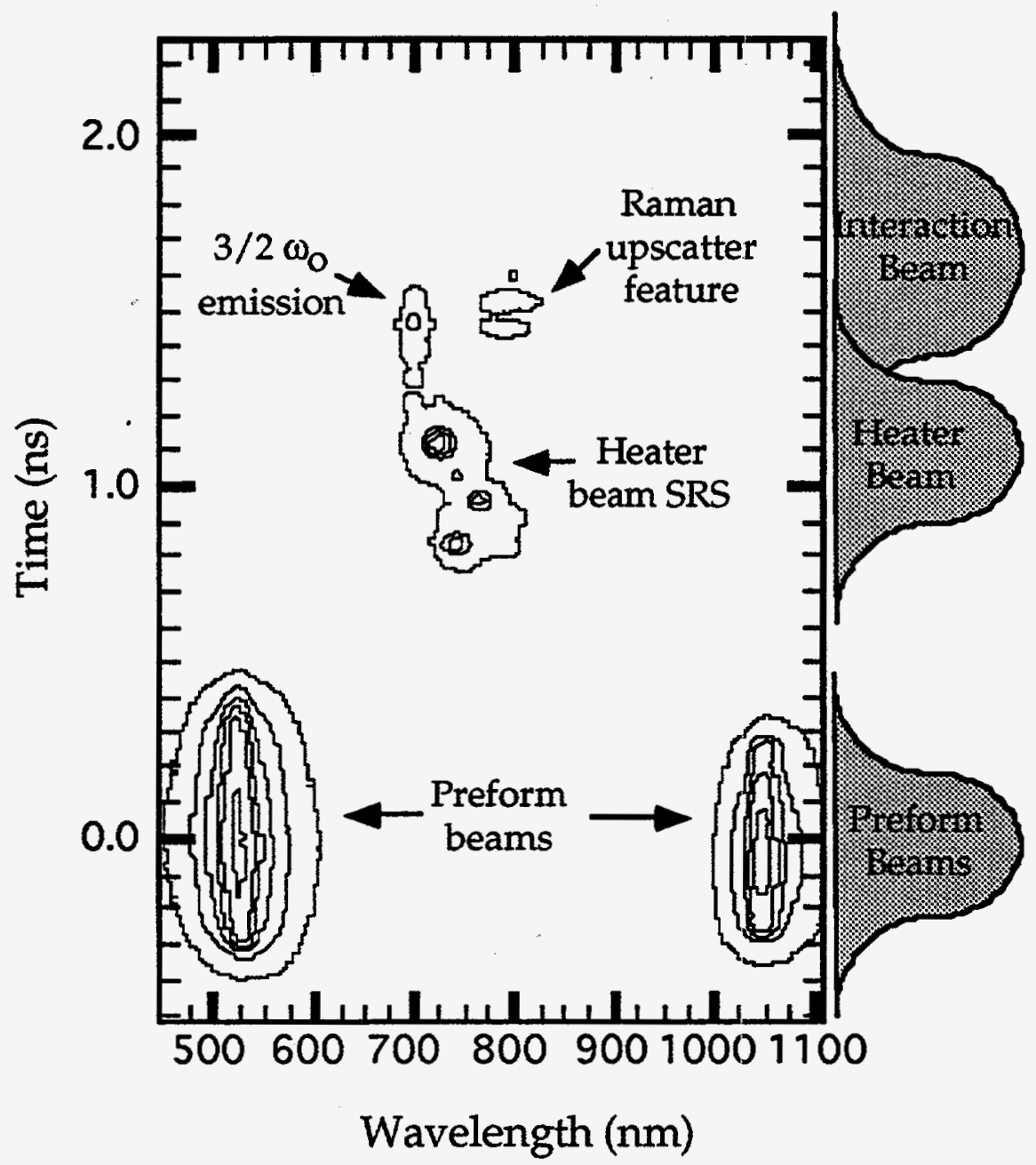

Figure 6.3.3 Spectrum from the heater beam stimulated Raman scattering diagnostic. Relative beam timing is drawn along the right side. The vertical axis represents relative time, while the horizontal axis shows the wavelength of the scattered light. 
half the damping on the Langmuir wave, vepwSRs, multiplied by the square root of the ratio of the light wave group velocity, $\mathrm{V}_{\mathrm{SSRS}}$, over the Langmuir wave's group velocity, $V_{\text {epwSRS, }}$ or $\gamma_{\text {OSRS }}>0.5 v_{\text {epwSRs }} \sqrt{V_{\text {sSRS }} / V_{\text {epwSRS }}}[106]$. As discussed in chapter 2, for a convective instability the homogeneous damping threshold depends upon the growth rate, YoSRS, being larger than the product of the Langmuir wave's damping, VepwSRS, and the scattered electromagnetic wave's damping, $v_{\text {SSRS }}$ or $\gamma_{\text {OSRS }}>\sqrt{v_{\text {SSRS }} v_{\text {epwSRS }}}$. Although the Landau damping on the Langmuir wave is increasing as density decreases, the light wave's damping is decreasing making the use of this diagnostic questionable in the case of convective instabilities.

Figure 6.3.4 a shows lines of constant $k \lambda_{\text {De }}$ for the Langmuir waves participating in resonant stimulated Raman scattering. These lines are plotted as a function of the wavelength of the scattered light detected in the experiment. The line labeled $k \lambda_{D e}=0.3$ shows that for a Langmuir wave to have a $k \lambda_{\mathrm{De}}=0.3$ and be in a resonant Raman process with a $700 \mathrm{~nm}$ scattered light wave that the electron temperature would have to be approximately 800 $\mathrm{eV}$. Likewise, for a Langmuir wave with a $\mathrm{k} \lambda_{\mathrm{De}}=0.3$ to be in a resonant Raman process with a $645 \mathrm{~nm}$ scattered light wave, the electron temperature would have to be approximately $400 \mathrm{eV}$. The Landau damping of the Langmuir wave as a function of the parameter $k \lambda_{\text {De }}$ is shown in figure $6.3 .4 \mathrm{~b}$. The gray line represents the analytic damping on the Langmuir wave valid for $k \lambda_{\mathrm{De}}<<1$. The solid line represents the numerical damping calculated solving the dispersion relation for Langmuir waves in the same manner as discussed in chapter 4 . For the parameters of this experiment, the threshold for absolute instability occurs for $k \lambda_{D e} \approx 0.25$. When this is used in conjunction 
with the cutoff of the Raman spectrum shown in figure 6.3.3, the estimated electron temperature at the time of the heater beam is approximately $600 \mathrm{eV}$ which is higher than the LASNEX prediction of $400 \mathrm{eV}$ shown in figure 6.3.2. One of the possible reasons for this discrepancy is that comparing the beam intensities above. The LASNEX simulation assumed an intensity of $2 \times 10^{13}$ $\mathrm{W} / \mathrm{cm}^{2}$ for the heater beam while in the experiment, the heater beam had an intensity of approximately $2 \times 10^{14} \mathrm{~W} / \mathrm{cm}^{2}$. This undoubtedly resulted in a higher temperature than the prediction of $400 \mathrm{eV}$.
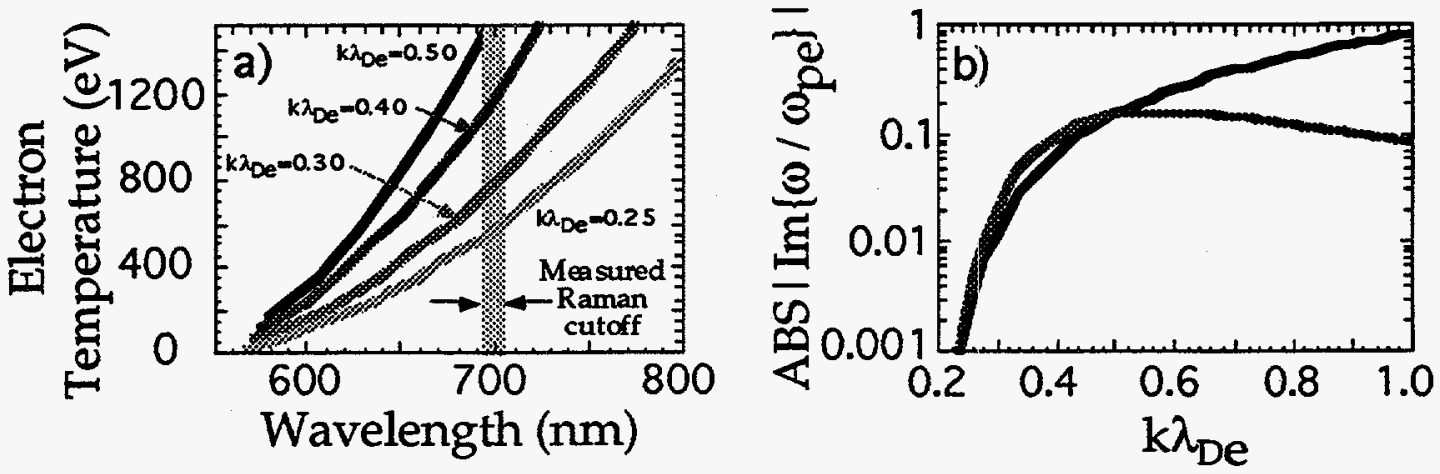

Figure 6.3.4 Concept behind the use of stimulated Raman scattering as a temperature diagnostic. Figure 6.3.4 a shows the corresponding temperature of the plasma such that a Langmuir wave with a given $k \lambda_{\text {De }}$ product participates in a resonant Raman scattering processes with a given wavelength scattered electromagnetic wave. Figure $6.3 .4 \mathrm{~b}$ displays the Landau damping of a Langmuir wave normalized to the real part of the frequency as a function of $k \lambda_{\text {De. }}$ Figure $6.3 .4 \mathrm{~b}$ includes Landau damping of the Langmuir wave only, although typically collisional damping becomes larger than Landau damping when $k \lambda_{D e}$ is less than 0.24 , however, this is dependent on the parameter $n_{o e} Z /\left(T_{e}\right)^{1.5}$. The solid black line represents the numerically 
calculated Landau damping while the gray line represents the analytic expression for the Landau damping which is valid for small $k \lambda_{D e}$.

This diagnostic can also be used in the case of stimulated Raman scattering from the $1.053 \mu \mathrm{m}$ interaction beam. In this case, the experiment showed that the short wavelength cutoff of the Raman spectrum occurred at approximately $1330 \mathrm{~nm}$ as shown in figure 6.4.1. The graph corresponding to figure 6.3.4 a for the case of the $1.053 \mu \mathrm{m}$ interaction beam is shown below in figure 6.3.5. In the case of the interaction beam, this diagnostic would predict a temperature for the plasma of 400 to $500 \mathrm{eV}$ during the time that the interaction beam is incident on the plasma.

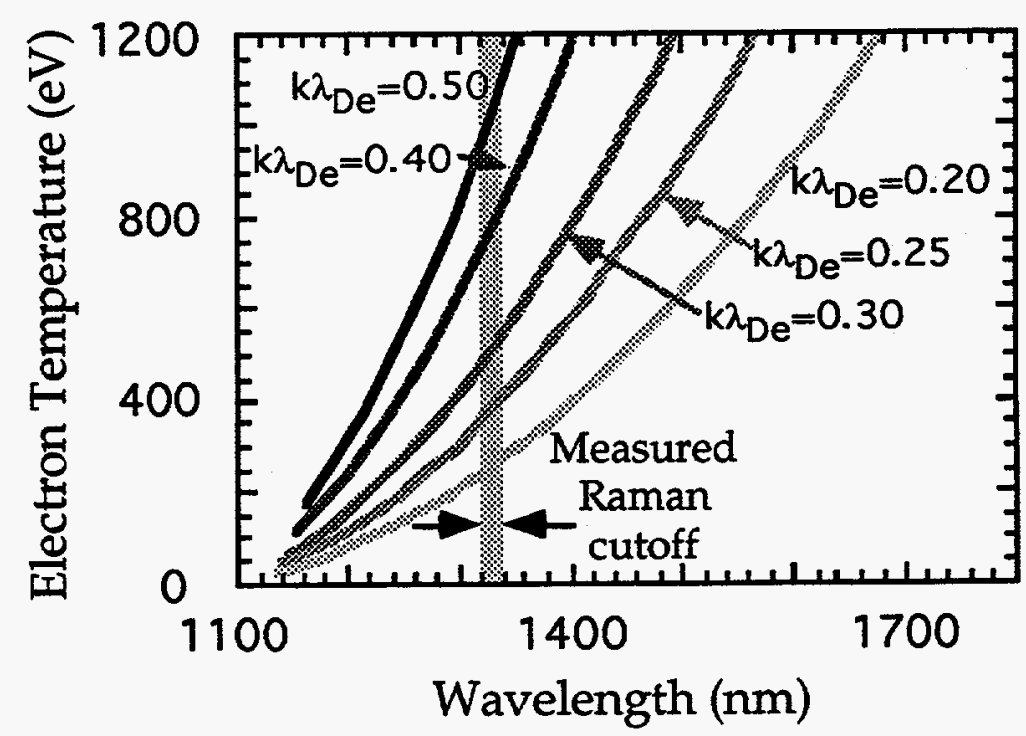

Figure 6.3.5 Corresponding temperature of the plasma such that a Langmuir wave with a given $k \lambda_{\text {De }}$ product participates in a resonant Raman scattering processes with a given wavelength scattered electromagnetic wave. This graph corresponds to Raman scattering with a $1.053 \mu \mathrm{m}$ interaction beam. 
The temperature of the plasma was measured by collective Thomson scattering from the assumed thermal level ion acoustic waves, the principles of which are discussed in detail in chapter 4[107]. Figure 6.3 .6 a below shows the Thomson scattering measurement from the ion acoustic waves while figure 6.3.6 b shows a lineout of the Thomson scattered spectrum which can be compared to figure 4.2.1 from chapter 4. The spectrum shown in figure 6.3.6 $\mathrm{b}$ has a peak to minimum of approximately 1.5 and a FWHM greater than the ion acoustic frequency as discussed in chapter 4. A comparison of these numbers with figure $4.2 .3 \mathrm{c}$ and $\mathrm{d}$ in chapter 4 shows that this spectrum exhibits too much broadening for any useful information concerning the ratio of the ion to electron temperature. Without any information regarding
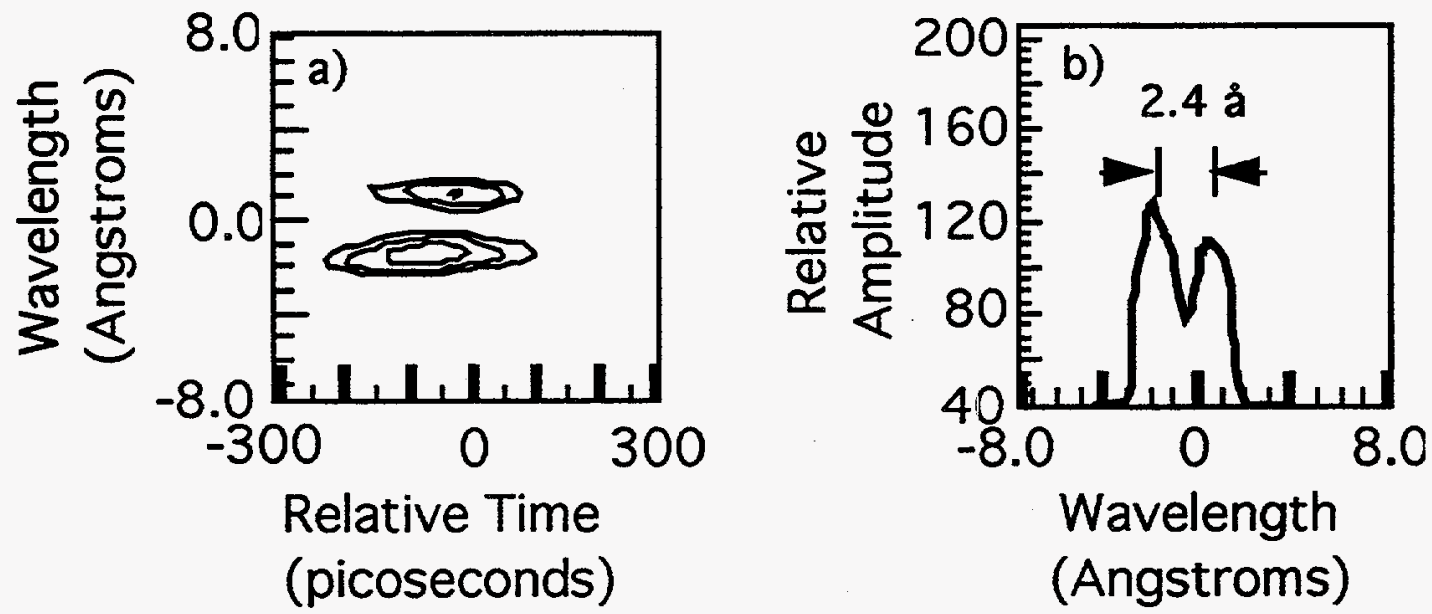

Figure 6.3.6 Thomson scattering spectrum taken with an interaction beam intensity of approximately $5 \times 10^{12} \mathrm{~W} / \mathrm{cm}^{2}$. Figure 6.3 .6 a displays the contour plots of the Thomson scattered spectrum showing the peak separation as a function of time. Figure $6.3 .6 \mathrm{~b}$ shows a lineout of the Thomson scattered spectrum which can be compared to the $\mathrm{CH}$ Thomson scattered spectrum generated in Chapter 4 in figure 4.2.1. 
the ratio of the ion to electron temperature there are considerable error bars on the estimation of the electron temperature due to the dependence of the spectrum on this ratio as can be seen in figure 4.2 .2 a of chapter 4 . This measurement would place the electron temperature at approximately $400 \mathrm{eV}$ with a $200 \mathrm{eV}$ uncertainty in either direction. In addition, this measurement was taken with the incident beam intensity of $5 \times 10^{12} \mathrm{~W} / \mathrm{cm}^{2}$. With a higher interaction beam intensity, the temperature of the plasma will increase as well.

The upscattering process shown in figure 6.3.3 has been observed in several experiments with different interpretations given as to the origins of the non-thermal-level Langmuir waves[63,108]. This process brings up the interesting point that it could be used as a temporal density diagnostic for thin foil $1 \mu \mathrm{m}$ interaction beam experiments. This could be very useful in diagnosing such experiments which typically must rely on time integrated measurements of the stimulated downscattering process. With this in mind, the short wavelength cutoff of the upscattering feature indicates that the maximum density near $t=1.52 \mathrm{~ns}$ is greater than $12 \%$ of the critical density, corresponding to a $k \lambda_{D e}$ of approximately 0.19 . Although there are higher densities present as indicated by the $3 / 2 \omega_{0}$ emission, the cutoff in this case is likely due to the cutoff of the $1 \mu \mathrm{m}$ Raman spectrum rather than the maximum density in the plasma. The wavelength response of the S-20 streak camera used in this experiment falls rapidly for wavelengths greater than 800 $\mathrm{nm}$. It is possible, therefore that the long wavelength cutoff of the upscatter feature is due to the streak camera, however, this cutoff of $820 \mathrm{~nm}$ also 
corresponds to $k \lambda_{\text {De }}$ of approximately 0.25 . With the use of a dedicated streak camera, the short wavelength cutoff of the upscattering feature could provide a useful measure of the maximum density present in the plasma as a function of time.

\subsection{Experimental results}

Measurements of the Langmuir wave spectra by Thomson scattering and the corresponding Raman spectrum are shown in figure 6.4.1. This figure was taken with a probe f/\# of approximately 3.33 which allowed for a large sampling of the wavenumber spectrum of the Langmuir waves. The interaction beam had an $\mathrm{f} / \#$ of approximately 6 which set the geometrical spread in the Thomson scattering angle of approximately 9.5 degrees. The angular spread measured in figure $6.4 .1 \mathrm{a}$ is close to approximately 8 degrees which is slightly less than the expected geometrical spread set by the assumption that the entire angular spread of the interaction beam is driving stimulated Raman scattering. In figure 6.4.1 a, the longer wavelength feature represents the Thomson downscattering from the waves that copropagate with the laser, referred to as SRS produced Langmuir waves[109]. The central feature at $351 \mathrm{~nm}$ is a combination of stray probe light and Thomson scattering from ion acoustic waves whose frequency shift is less than the resolution of the spectrometer. The shorter-wavelength feature represents Thomson scattering from Langmuir waves traveling opposite to the laser wavevector, referred to as counterpropagating Langmuir waves. These counterpropagating Langmuir waves have been interpreted as due to the 

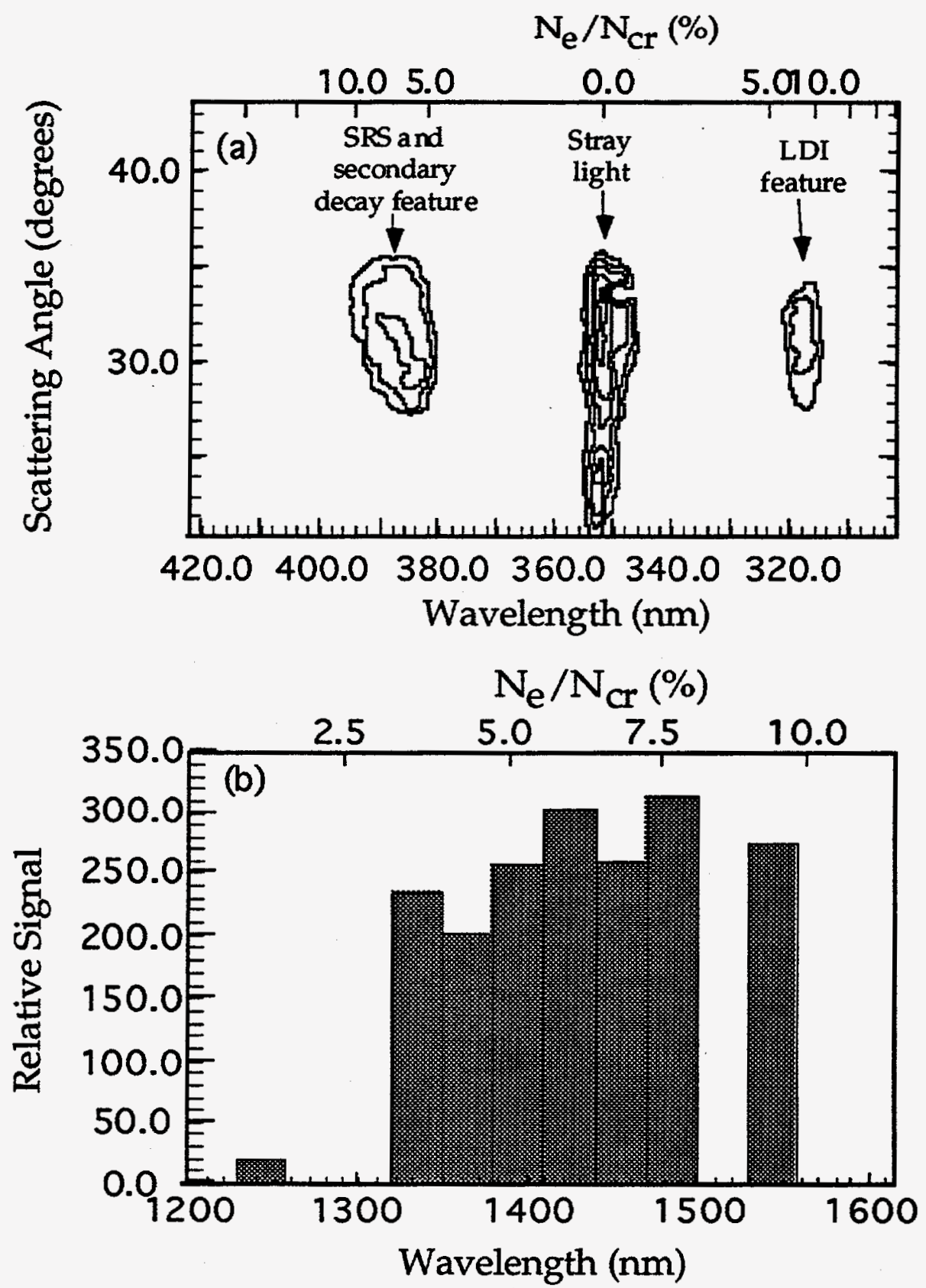

Figure 6.4.1 Thomson scattering measurement of the Langmuir wave spectrum using a $f / 3.3$ probe beam (a) and the backscattered electromagnetic waves (b) driven by the $1 \mu \mathrm{m}$ interaction beam. 
Langmuir decay instability, as discussed below. Figure $6.4 .1 \mathrm{~b}$ shows the corresponding time-integrated measurement of the scattered electromagnetic wave driven by stimulated Raman scattering. In figure $6.4 .1 \mathrm{~b}$, the channels centered at 1275, 1305, and $1510 \mathrm{~nm}$ were not functional.

For the purpose of comparing the different processes observed in the experiment it is useful to look at the density range over which they occurred. In figure 6.4.1, the Thomson downscatter feature, assuming an electron temperature of $500 \mathrm{eV}$, extends from $n_{e} / n_{c r}=0.045$ to $n_{e} / n_{c r}=0.11$, corresponding to values of $k \lambda_{\text {De }}$ ranging from 0.24 to 0.14 , respectively. Likewise, the Thomson upscatter feature in figure 6.4.1 extends from $n_{e} / n_{C r}=0.07$ to $n_{e} / n_{C r}=0.11$, corresponding to values of $k \lambda_{D e}$ ranging from 0.19 to 0.14 , respectively. The Raman upscatter feature shown in figure 6.3.3 extends from $n_{e} / n_{C r}=0.065$ to $n_{e} / n_{C r}=0.12$, corresponding to values of $k \lambda \lambda_{D e}$ ranging from 0.25 to 0.12 , respectively.

Figure 6.4.2 displays the results from a separate shot in which an aperture was placed in front of the Thomson probe beam resulting in a $\mathrm{f} / \#$ of approximately 25 . In this case, the geometrical spread in the Thomson scattering angle was determined by the $\mathrm{f} / \#$ of the probe beam which produced an angular spread of approximately 2.3 degrees. The angular spread measured in figure 6.4.2 is approximately $2-4$ degrees for the SRS feature. The explanation of the Thomson scattered features in figure 6.4.2 is the same as discussed for figure 6.4.1. In this case the measured signal was just above the detection threshold of the detector which resulted in a reduced signal-to-noise ratio. An angular broadening of 2 degrees would indicate a broadening in the wavenumber spectrum of the Langmuir waves of approximately 5.5 percent. 
The modulational instability would predict a broadening in the Langmuir wave spectrum proportional to the Langmuir wave amplitude for $k_{e p w} \lambda_{D e} \approx 0.2[49]$. For $k_{e p w} \lambda_{D e} \approx 0.2$, a broadening in the Langmuir wave spectrum of $5.5 \%$ would suggest a Langmuir wave amplitude of approximately $n_{e p w} / n_{o e} \approx 6.5 \%$, which is in reasonable agreement with the estimation given below in section 6.5. However, as discussed in chapter 5, the broadening of the Langmuir wave spectrum in that experiment did not appear to be correlated with the Langmuir wave amplitude as measured by the intensity of the Thomson scattering spectrum.

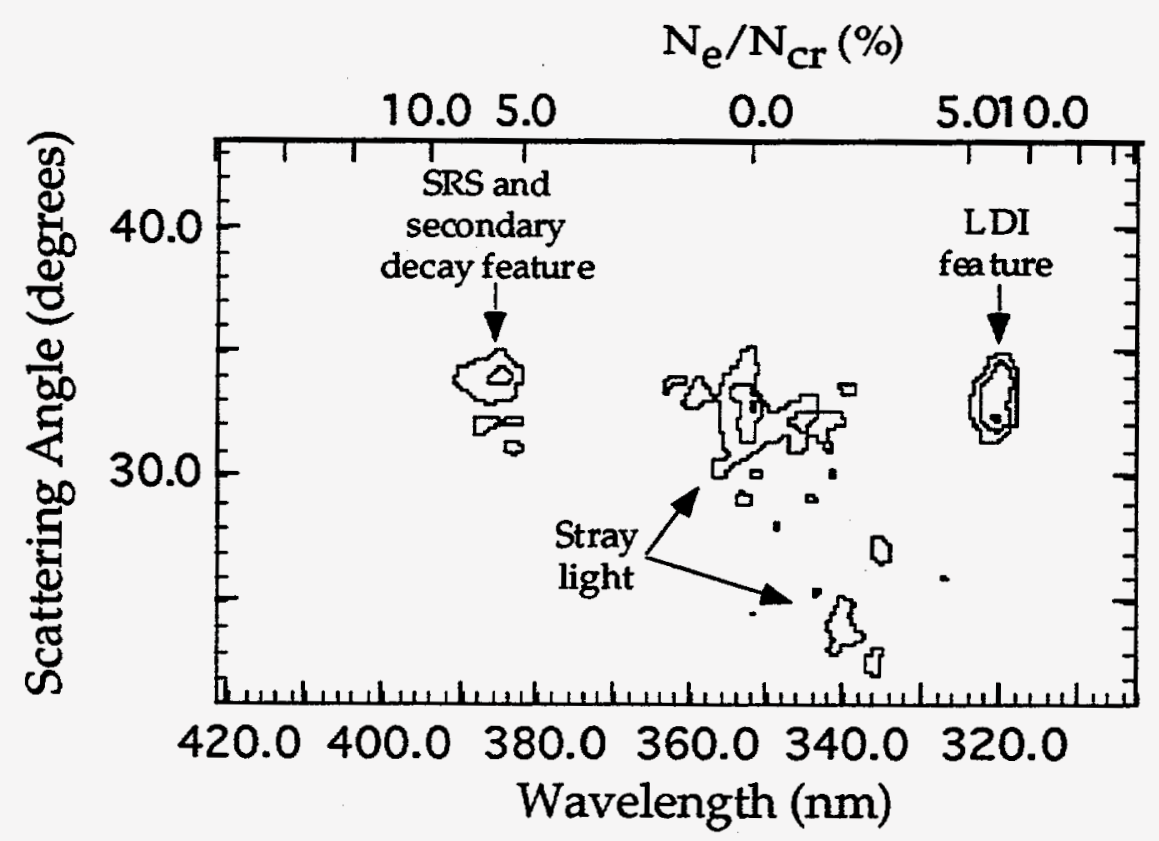

Figure 6.4.2 Thomson scattering measurement of the Langmuir wave spectrum using a f/ 25 probe beam. The vertical axis represents the scattering angle relative to the center of the aperture on the probe beam. The bottom horizontal axis represents the wavelength of the Thomson scattered signals while the top horizontal axis shows the approximate percent of critical density corresponding to the frequency shift of the Thomson scattered signal. 
One of the more interesting features observed in figure 6.3 .3 was the signal level of the Raman upscatter and $3 / 2 \omega_{0}$ emission. These different processes are both attributed to Thomson scattering of the incident laser from Langmuir waves, although the explanations for these processes differ in the source and mechanisms responsible for the Langmuir waves as discussed below and in chapter 3. These two features have the same approximate spectral energy, showing that although the Langmuir waves are driven at different densities, the amplitude of the Langmuir waves being scattered from have approximately the same magnitude.

With the addition of a random phase plate, Thomson scattered signals were observed on only one shot in which the stimulated Raman signal from the interaction beam saturated the Germanium detector array. This shot did not show any evidence of the Raman upscatter feature even though there was a short $3 / 2 \omega_{0}$ emission feature near the beginning of the interaction beam. The heater beam Raman diagnostic indicated that the maximum density fell below approximately $0.2 \mathrm{n}_{\mathrm{e}} / \mathrm{n}_{\mathrm{cr}}$ early on in the interaction beam in contrast to the data shown in figure 6.3.3. The lack of a quarter critical surface makes it more difficult for the enhanced Thomson model, which was also discussed in chapter 5, to explain either the Thomson upscatter feature seen in figure 6.4.1 a or the Raman upscatter feature shown in figure 6.3.3. The absence of the Raman upscatter feature in this case could be attributed to a number of reasons ranging from secondary effects of the random phase plates to the absence of the quarter critical surface. It would be very interesting to look at the effect of Random phase plates on the Raman upscatter feature 
relative to the stimulated downscatter process in an attempt to better understand the Raman upscatter feature.

\subsection{Discussion}

The measured ratio of the amplitude of the SRS Langmuir wave, $\mathrm{n}_{\text {epwSRs }} / \mathrm{n}_{\mathrm{Oe}}$, to the amplitude of the counterpropagating Langmuir waves, $\mathrm{n}_{\text {epwLDI }} / \mathrm{n}_{\mathrm{Oe}}$, is $0.5 \pm 0.2$. The coupled equations describing SRS and the Langmuir decay instability[9] can be used to infer the relationship between the amplitudes of the participating waves. With a $\mathrm{CH}$ plasma, the Landau damping on the ion acoustic waves is approximately ten percent of the ion acoustic frequency[110]. The strong damping approximation reduces the ion wave equation to an algebraic equation relating the ion wave amplitude to the product of the Langmuir pump and daughter wave amplitudes,

$$
\frac{\mathrm{n}_{\mathrm{iaLDI}}}{\mathrm{n}_{\mathrm{oe}}} \approx \frac{1}{2}\left(\frac{\omega_{\mathrm{ia}}}{v_{\mathrm{ia}}}\right) \frac{1}{\mathrm{k}_{\mathrm{epwSRS}}^{2} \lambda^{2}}\left(\frac{\mathrm{n}_{\mathrm{epwSRS}}}{\mathrm{n}_{\mathrm{oe}}} \frac{\mathrm{n}_{\mathrm{epwLDI}}}{\mathrm{n}_{\mathrm{oe}}}\right),
$$

where kepwSRS is the SRS Langmuir wavenumber, niaLDI/noe is the ion wave amplitude, $\lambda_{\mathrm{De}}$ is the Debye length, and $\omega_{\mathrm{ia}} / v_{\mathrm{ia}}$ is the ratio of the real part of the frequency to the damping of the ion acoustic wave.

The Langmuir decay instability can saturate the Raman process by increasing the damping on the Langmuir wave[9], driving the Raman process below threshold for absolute instability. The threshold for absolute instability is given by 


$$
\begin{aligned}
& \gamma_{\text {oSRS }}>0.5 v_{\text {epwSRS }} \sqrt{\frac{V_{\text {SSRS }}}{V_{\text {epwSRS }}} \text { with }} \\
& v_{\text {epwSRS }} \approx 0.5 \omega_{\mathrm{pe}}\left(\frac{n_{\text {iaLDI }}}{n_{\text {oe }}} \frac{n_{\text {epwLDI }}}{n_{\text {oe }}}\right),
\end{aligned}
$$

where $\omega_{\text {pe }}$ is the plasma frequency, $\gamma_{\text {OSRS }}$ is the homogeneous growth rate for Raman scattering and $V_{S S R S}$ and $V_{e p w S R S}$ are the group velocities of the Raman scattered light wave and SRS Langmuir wave respectively. The damping is due primarily to the pump depletion term in the SRS Langmuir wave equation. Upon combining equation 6.5 .1 and 6.5 .2 in conjunction with the measured ratio of the pump Langmuir wave and the counterpropagating Langmuir wave, the SRS Langmuir wave's amplitude was inferred to be $n_{\text {epw }} / n_{0 e}=5.0 \%$,

In order for the Langmuir decay instability to explain the results of this experiment, this amplitude must be above the homogeneous damping threshold for LDI[39],

$$
\gamma_{\text {OLDI }}>\sqrt{v_{\text {epwLDI }} v_{\text {iaLDI }}} \Rightarrow \frac{n_{\text {epwSRS }}}{n_{\mathrm{oe}}}>4 k_{\text {epwSRS }} \lambda_{\text {De }} \sqrt{\frac{v_{\text {epwLDI }} \frac{v_{\text {iaLDI }}}{\omega_{\text {ia }}}}{\omega_{\text {pe }}}}
$$

where $\gamma_{\text {OLDI }}$ is the homogeneous growth rate for the Langmuir decay instability and $v_{\text {epw }}$ is the damping on the counterpropagating Langmuir wave. Ignoring the damping effects of cascading, the homogeneous damping threshold for the Langmuir decay instability near seven percent of critical is approximately 0.005 . This amplitude is well below the value obtained above 
suggesting that the Raman Langmuir wave was above the damping threshold for Langmuir decay.

Other explanations for the counterpropagating Langmuir waves have been considered. The most straightforward way of producing counterpropagating Langmuir waves involves the propagation of the Langmuir waves produced by stimulated Raman scattering up the density gradient to their reflection point and back. This process, as pointed out in chapter 3, results in too much damping of the Langmuir waves to explain the observed ratio of amplitudes. Another mechanism involving the reflection of Langmuir waves from their critical surface is the direct reflection of Langmuir waves from an ion wave whose amplitude is sufficient to cause the local plasma density to become overdense to the plasma wave. While very large ion waves might in principle produce densities large enough to directly reflect SRS Langmuir waves, this would require large ion wave amplitudes $(\sim 10 \%)$ which would likely quench the SRS itself[56].

Another mechanism capable of producing counterpropagating Langmuir waves involves the quasiresonant mode coupling of Langmuir waves with ion waves[62,64]. In this process a Langmuir wave can couple with an ion wave generating Langmuir waves which have a wavevector, $\mathrm{k}_{\mathrm{L}}$, equal to the original Langmuir wavevector, $\mathrm{k}_{\mathrm{epw}}$, plus an integer number of the ion wave's wavevector or $\vec{k}_{L}=\vec{k}_{\text {epw }} \pm n k_{i o n} \hat{x}$. For this process to be efficient, the resultant mismatch between the Langmuir waves must be less than the frequency variation, $\Delta \omega$, produced by the ion wave, $\Delta \omega / \omega_{\mathrm{pe}}=0.5 \mathrm{n}_{\mathrm{ia}} / \mathrm{n}_{\mathrm{oi}}[62]$. Assuming that the two Langmuir wave features differed by an ion acoustic frequency, their frequency mismatch would be 
approximately $\Delta \omega / \omega_{\mathrm{pe}}=4 \sqrt{\mathrm{Zm_{ \textrm {e } }} / \mathrm{M}_{\mathrm{i}}} \mathrm{k} \lambda_{\mathrm{De}} \approx 0.1 \mathrm{k} \lambda_{\mathrm{De}}$. Therefore, for this process to be efficient the ion wave fluctuation would have to be approximately $4 \%$ based on the parameters inferred from the above measurements. In addition, the Thomson scattered data from the counterpropagating Langmuir waves had nearly the same magnitude wavevector as the copropagating Langmuir waves, the two wavevectors being antiparallel. The ion wave which provided this quasiresonant coupling would be required to have a wavevector equal to $\overrightarrow{\mathrm{k}}_{\text {ion }}=2 \overrightarrow{\mathrm{k}}_{\text {epw }} / \mathrm{n}$, where $\mathrm{n}$ is an integer. The Thomson scattered data shown in figure 6.4.1 indicates that the copropagating Langmuir waves observed in the experiment ranged from $1.7 \overrightarrow{\mathrm{k}}_{\mathrm{o}}$ at the lower densities to $1.6 \overrightarrow{\mathrm{k}}_{\mathrm{o}}$ at the highest density where $k_{0}$ is the wavenumber of the interaction beam. That would suggest that the ion waves would require a wavenumber between $3.4 \vec{k}_{0} / n$ and $3.2 \overrightarrow{\mathrm{k}}_{\mathrm{o}} / \mathrm{n}$. The source of ion waves typically proposed for this process is stimulated Brillouin scattering[64]. Only extreme forward scatter of SBS could satisfy the wavenumber conditions above. In that case $n$ would become very large and consequently this process would drive a broad range of Langmuir waves only a small fraction of which would be observed as the counterpropagating feature. Again the measured difference between these signals precludes this possibility.

The enhanced Thomson scattering model[67,96] would predict Langmuir waves traveling in both directions of the incident laser. According to the enhanced Thomson scattering model, Langmuir waves are produced by the bump-on-tail instability between hot electrons and the background electron distribution. The electromagnetic wave attributed to stimulated Raman scattering would then represent the incident pump beam scattering 
off these enhanced plasma waves. As discussed in chapter 5 , the enhanced Thomson scattering model would, however, predict a much broader spectrum of Langmuir waves than observed in this experiment. In addition, the Thomson scattered signal from the counterpropagating Langmuir waves was unaffected by the absence of the quarter critical surface indicating that the enhanced Thomson scattering model was not responsible for the production of the counterpropagating Langmuir waves.

\subsection{Summary}

In summary, the Langmuir-wave spectra measured by Thomson scattering show that Langmuir waves are present which propagate both parallel and antiparallel to the laser wavevector. The Langmuir waves propagating parallel to the laser's wavevector are expected as they are being driven directly by stimulated Raman scattering. The counterpropagating waves are not predicted directly from the Raman process itself. A number of mechanisms were reviewed as possible candidates for the production of such waves, however, as discussed in section 6.5 most of these processes could not explain the data observed in the experiment. The presence and properties of the Langmuir waves traveling antiparallel to the incident laser's wavevector can be explained by the Langmuir decay of the Langmuir waves traveling parallel to the laser's wavevector, but not by any other mechanism identified in section 6.5. This thus represents the first experimental observation of the Langmuir decay instability. 



\section{Chapter 7}

\section{Summary}

The saturation of stimulated Raman scattering, as well as other instabilities, is a difficult problem which will require a great deal of effort in experiments, theory, and simulations to understand all of the mechanisms at work and their relative importance. This dissertation dealt primarily with the experimental observation of the Langmuir decay instability and identified a number of possible experiments which could be used to look for other saturation mechanisms. The following paragraphs give a summary of the results obtained in the previous chapters of this dissertation.

Chapter 1 reviewed a number of applications in which Raman scattering was an important process. The success or optimization of these applications is dependent on the saturation of the Raman process. However, very little experimental work has been done specifically considering what mechanisms are occurring and contributing to the saturation of Raman scattering. The goal of this dissertation was to evaluate and observe these saturation 
mechanisms. The experiments performed for this dissertation, described in chapters 5 and 6, looked specifically for the Langmuir decay instability described in section 2.5 .

Chapter 2 provided an introduction to a number of saturation mechanisms and in some cases experimental evidence for the occurrence of these mechanisms. It also attempted to show cases when a particular saturation mechanism did not explain certain details from experimental data, indicating that the mechanism was not responsible for the saturation of the amplitude of the daughter waves in that case. The saturation of stimulated Raman scattering is clearly dependent on the intensity regime. For example, in the short-pulse high-intensity regime, the reflectivity of the Raman scattered light was found to be close to ten percent[37]. In contrast, for comparable plasmas in the long-pulse moderate-intensity regime, the reflectivity would likely saturate at a value closer to 0.1 to 0.01 percent of the laser energy.

In the long-pulse regime, the saturation mechanisms responsible for limiting the amplitude of the daughter waves depend on a number of parameters. The saturation mechanisms affecting the Langmuir waves tend to group most easily by the parameter $k \lambda_{D e}$, which is simply the thermal velocity of the plasma divided by the phase velocity of the Langmuir wave. Convective instabilities such as forward Raman scattering can saturate by plasma inhomogeneity at low laser intensities, however, for higher laser intensities other saturation mechanisms limit the amplitude of the daughter waves. For large values of $k \lambda_{D e}$, greater than 0.3 to 0.4 , saturation mechanisms such as particle trapping or wave breaking are likely candidates 
which is consistent with experimental results such as shown in figure 2.10.3. For values of $k \lambda_{\text {De }}$ between 0.3 to 0.05 , saturation of the daughter wave amplitudes is likely dominated by wave-wave saturation mechanisms such as the Langmuir decay instability or the electromagnetic decay instability as discussed in chapter 6 and 3 , respectively. This is supported by the experiments described in chapter 6 which observed the Langmuir decay instability over the range of $k \lambda_{\text {De }}$ from approximately 0.25 to 0.15 , as well as by numerous simulations. For smaller values of $k \lambda_{D e}$, less than 0.05 , modulational instability and collapse are predicted from simulations to dominate the saturation of stimulated Raman scattering. As shown in section 2.6, the broadening in the spectrum of the Langmuir waves due to the modulational instability increases rapidly for $k \lambda_{\text {De }}$ less than 0.1 which is qualitatively consistent with simulations.

The experiments performed for this dissertation involved the saturation of Raman scattering in the long-pulse regime, however, simulations and experiments performed in the short pulse regime allow some deductions to be made. For short pulses at moderate intensities, the saturation of the Raman process likely results from the limited gain obtainable from the pulse width itself. For higher intensities, however, the daughter waves have ample time to grow to large amplitudes. In this shortpulse high-intensity regime, particle-in-cell simulations have shown that the saturation level of stimulated Raman scattering is not dependent on ion dynamics[111]. This indicates that the Raman saturation is not dominated by parametric instabilities involving ion waves or by mode coupling or pump depletion resulting from stimulated Brillouin scattering. The saturation 
likely resulted from mechanisms which did not depend on ion waves, such as particle trapping, wave breaking, relativistic effects, or pump depletion which were discussed in section $2.10,2.9$, and 2.4 respectively. In chapter 2 , an experiment was proposed to verify the saturation of Raman due to particle trapping or wave breaking in this regime.

Chapter 3 examined the electromagnetic decay instability, specifically looking for evidence that this instability is occurring in laser-produced plasmas. The most compelling evidence that the electromagnetic decay instability occurs in laser-produced plasmas comes from $\omega_{0} / 2$ emission observed in experiments. This $\omega_{0} / 2$ emission had been attributed to Thomson scattering and inverse resonance absorption in previous papers. It was shown in chapter 3 that the ratio of energy between the $3 \omega_{0} / 2$ emission and the $\omega_{0} / 2$ emission measured in previous experiments was inconsistent with the interpretation that the $\omega_{0} / 2$ emission from these experiments was the result of Thomson scattering. In chapter 3, and in previous work, it was shown that without significant perturbations to the background density distribution, inverse resonance absorption could not explain the levels of $\omega_{0} / 2$ emission measured in the experiments discussed in chapter 3. To explain the levels of $\omega_{0} / 2$ emission, inverse resonance absorption, therefore, requires hotspots or large level ion fluctuations to convert a larger phase space of Langmuir waves into electromagnetic emission. In addition, the observed $\omega_{\mathrm{O}} / 2$ spectra showed a slight red shift which is not predicted by inverse resonance absorption. Experiments measuring hot electrons have shown that enough energy is contained in the two plasmon decay daughter waves to account for the $\omega_{0} / 2$ emission. By using the electromagnetic decay 
instability to explain $\omega_{0} / 2$ emission, the relative levels of $3 \omega_{0} / 2$ emission and $\omega_{0} / 2$ emission can be explained by attributing these features to independent mechanisms, using the electromagnetic decay instability to explain the $\omega_{0} / 2$ emission. In addition, the electromagnetic decay instability also allows for a slight red shifting of the $\omega_{0} / 2$ spectrum seen in the experiments and is consistent with the amount of energy found in the $\omega_{0} / 2$ feature.

Near the critical surface, Langmuir waves and ion acoustic waves can generate transverse currents which drive electromagnetic waves close to the frequency of the incident laser. In chapter 3 , a model was constructed which shows the ratio of the vector potential resulting from the coupling between Langmuir and ion acoustic waves driven by the ion acoustic decay instability relative to the vector potential driven by Thomson scattering of the incident pump from thermal level ion acoustic waves. It was shown that these electromagnetic waves can be several orders of magnitude higher than Thomson scattering from thermal levels. They can, therefore, provide an enhanced noise source from which stimulated Brillouin scattering can grow.

Chapter 3 also examined the conditions under which the electromagnetic decay instability could be driven absolute. The electromagnetic decay instability can be driven absolute when its daughter electromagnetic wave is driven perpendicular to the density gradient and also when the pump Langmuir wave is sufficiently localized. For the case of a Raman generated Langmuir wave, these conditions were obtained from previous calculations of the Langmuir wave localization distance[78] and the general condition for absolute instability given a localized pump[28,87]. In addition, the electromagnetic decay instability can also be driven absolute due 
to the coupling of the daughter wave energy back into the interaction region which can occur when the Raman process is being driven primarily by hotspots in the laser. For moderate hotspot intensities, the daughter electromagnetic wave can become trapped in the resultant density cavern and consequently couple back into the interaction region driving the instability absolute.

Chapter 4 presented a review of Thomson scattering in homogeneous plasmas and then looked at the effects of inhomogeneities on the Thomson scattered vector potential. It also discussed the implications which inhomogeneities introduced into the interpretation of Thomson scattering experiments. The inhomogeneities primarily caused a broadening in the spectrum limiting the information which could be extracted from the Raman spectrum.

Chapter 5 described an experiment which was used to distinguish between several models for the electromagnetic emission between $\omega_{0} / 2$ and $\omega_{0}$ usually attributed to stimulated Raman scattering. The alternative model used to explain this emission is known as the enhanced Thomson scattering model. In the experiment described in chapter 5, Thomson scattering was used to measure the Langmuir wave spectrum associated with Raman backscatter. This measurement showed that the Langmuir waves obeyed the dispersion relation for Langmuir waves driven by stimulated Raman scattering. This experiment found that the Thomson scattered signal was observed over a narrow angular range which was consistent with the assumption that the Langmuir waves were driven by stimulated Raman scattering and inconsistent with the assumption that they were driven by the 
bump-on-tail instability predicted in the enhanced Thomson scattering model. The two plasmon decay instability was driven in solid Carbon target experiments, as inferred from measurements of $3 / 2 \omega_{0}$ emission. Stimulated Raman scattering, however, was not detected in these experiments which retained a quarter-critical surface for the entire duration of the interaction beam. The lack of stimulated Raman scattering was expected due to the low convective gain resulting from the relatively steep density gradient. The enhanced Thomson scattering model, however, would predict stimulated Raman scattering in this case due to the presence of two plasmon decay. Therefore, the lack of stimulated Raman scattering in the solid target experiments shows that the Langmuir waves were not driven sufficiently by the bump-on-tail instability to be measured.

Chapter 6 described an experiment which was designed to look for one particular saturation mechanism of stimulated Raman scattering known as the Langmuir decay instability. Again the Langmuir-wave spectra were measured by Thomson scattering. In this experiment, the Thomson scattering measurements showed that Langmuir waves were present which propagated both parallel and antiparallel to the laser wavevector. The Langmuir waves propagating parallel to the laser's wavevector were directly driven by stimulated Raman scattering, however, the counterpropagating waves are not predicted directly from the Raman process itself. These waves require another mechanism to reverse the wavenumber of the Langmuir waves directly driven in the Raman process itself. A number of mechanisms were reviewed as possible candidates for the production of such waves, however, as discussed in section 6.5, most of these processes could not explain the data 
observed in the experiment. The presence and properties of the Langmuir waves traveling antiparallel to the incident laser's wavevector can be explained by the Langmuir decay of the Langmuir waves traveling parallel to the laser's wavevector, but not by any other mechanism identified in section 6.5. This measurement provided the first experimental observation of the Langmuir decay instability and provided a measurement of the relative energy contained in the counterpropagating Langmuir waves. 


\section{Appendix 2.1}

\section{Derivation of equations describing stimulated Raman scattering}

This appendix reviews the derivation of the equations describing the three waves participating in stimulated Raman scattering.

The first step is to derive the equation describing the growth of the Thomson scattered vector potential[16]. The derivation begins with Ampere's law

$$
\nabla \times \vec{B}=\frac{4 \pi}{c} \vec{J}+\frac{1}{c} \frac{\partial \vec{E}}{\partial t}
$$

which relates the magnetic field, $\overrightarrow{\mathrm{B}}$, to the current, $\overrightarrow{\mathrm{J}}$, and the rate of change of the electric field, $\vec{E}$. After inserting the relations $\vec{B}=\nabla x \vec{A}$, and $\vec{E}=-\nabla \phi-\frac{1}{c} \frac{\partial \vec{A}}{\partial t}$ above, equation 2.1.1 may be expressed as

$$
\frac{\partial^{2} \overrightarrow{\mathrm{A}}}{\partial \mathrm{t}^{2}}-c^{2} \nabla^{2} \overrightarrow{\mathrm{A}}=4 \pi c \overrightarrow{\mathrm{J}}-c^{2} \nabla\left(\nabla \cdot \overrightarrow{\mathrm{A}}+\frac{1}{c} \frac{\partial \phi}{\partial \mathrm{t}}\right)
$$


relating the vector potential, $\vec{A}$, to the current and the scalar potential, $\phi$. The transverse component of the scattered vector potential, $\vec{A}_{t}$, can be written as

$$
\frac{\partial^{2} \overrightarrow{\mathrm{A}}_{\mathrm{t}}}{\partial \mathrm{t}^{2}}-c^{2} \nabla^{2} \overrightarrow{\mathrm{A}}_{\mathrm{t}}=4 \pi c \vec{J}_{\mathrm{t}}
$$

The transverse current, $\overrightarrow{\mathrm{J}}_{t}$, is found from the electron momentum equation

$$
\begin{aligned}
& \frac{\partial \overrightarrow{\mathrm{u}}}{\partial \mathrm{t}}+\frac{1}{2} \nabla(\overrightarrow{\mathrm{u}} \cdot \overrightarrow{\mathrm{u}})-\overrightarrow{\mathrm{u}} \mathrm{x}(\nabla \times \overrightarrow{\mathrm{u}})= \\
& \frac{-\mathrm{e}}{\mathrm{m}_{\mathrm{e}}}\left[-\nabla \phi-\frac{1}{\mathrm{c}} \frac{\partial \overrightarrow{\mathrm{A}}}{\partial \mathrm{t}}+\frac{\overrightarrow{\mathrm{u}}}{\mathrm{c}} \times(\nabla \times \overrightarrow{\mathrm{A}})\right]-\frac{\nabla \mathrm{p}_{\mathrm{e}}}{\mathrm{n}_{\mathrm{e}} \mathrm{m}_{\mathrm{e}}},
\end{aligned}
$$

without damping, where $\vec{u}, p_{e}$, and $n_{e}$ are the electron fluid velocity, pressure, and density respectively. By inspection of equation 2.1.4, the transverse electron fluid velocity, $\vec{u}_{t}$, is equal to $\vec{u}_{t}=e \vec{A} / \mathrm{cm}_{e}$ where e and $m_{e}$ are the magnitude of the electron's charge and mass respectively. The scattered vector potential can then be written as

$$
\frac{\partial^{2} \overrightarrow{\mathrm{A}}_{t}}{\partial \mathrm{t}^{2}}-c^{2} \nabla^{2} \overrightarrow{\mathrm{A}}_{\mathrm{t}}=-\frac{4 \pi \mathrm{e}^{2}}{\mathrm{~m}_{\mathrm{e}}} \mathrm{n}_{\mathrm{e}} \overrightarrow{\mathrm{A}}_{\mathrm{t}}
$$

The electron fluid density, $n_{\mathbf{e}}$, can be expressed as a zero order background density, $n_{O e}$, and a first order perturbation, $n_{\text {epw }}$, representing the Langmuir wave fluctuation present in the plasma. Next the transverse vector potential, $\vec{A}_{t}$, can be separated into components representing the interaction beam, $A_{0}$, and the vector potential of the scattered electromagnetic wave, Ascatt. The 
resulting equation describing the growth of the scattered vector potential, without damping, is

$$
\begin{aligned}
& \left(\frac{\partial^{2}}{\partial t^{2}}+\omega_{\mathrm{pe}}^{2}-c^{2} \nabla^{2}\right) \frac{q A_{\text {scatt }}}{m_{e} c^{2}}= \\
& -\frac{4 \pi n_{o e} e^{2}}{m_{e}}\left(\hat{e}_{s c a t t} \cdot \hat{e}_{o}\right) \frac{n_{e p w}}{n_{o e}} \frac{q A_{o}}{m_{e} c^{2}},
\end{aligned}
$$

where $\hat{e}_{\text {scatt }}$ and $\hat{e}_{\mathrm{o}}$ are unit vectors in the direction of the scattered and interaction vector potentials, respectively. The electron fluid momentum equation, equation 2.1.4, also contains the longitudinal component of the electron fluid representing the Langmuir wave. The longitudinal electron velocity is given by

$$
\frac{\partial \vec{u}_{L}}{\partial t}+\frac{1}{2} \nabla(\vec{u} \cdot \vec{u})=-\frac{e \vec{E}_{L}}{m_{e}}-\frac{\nabla p_{e}}{n_{e} m_{e}}
$$

Equation 2.1.7 can used in conjunction with the continuity equation for the electron fluid,

$$
\frac{\partial n_{e}}{\partial t}+\nabla \cdot\left(n_{e} \vec{u}\right)=0.0
$$

to obtain the equation describing the growth of the Langmuir wave. Combining a partial with respect to $t$ of equation 2.1.8 and a divergence of equation 2.1.7 produces 


$$
\begin{aligned}
& \frac{\partial^{2} n_{e}}{\partial t^{2}}-\frac{e n_{e} \nabla \cdot \vec{E}_{L}}{m_{e}}-n_{e} \nabla \cdot\left(\frac{\nabla p_{e}}{n_{e} m_{e}}\right)= \\
& \frac{1}{2} n_{e} \nabla^{2}(\vec{u} \cdot \vec{u})-\frac{\partial n_{e}}{\partial t} \nabla \cdot \vec{u}-\nabla n_{e} \cdot \frac{\partial \vec{u}}{\partial t}-\vec{u} \cdot \frac{\partial \nabla n_{e}}{\partial t} .
\end{aligned}
$$

The pressure, $\mathrm{pe}_{\mathrm{e}}$, is assumed to follow the one dimensional adiabatic equation of state, $\mathrm{p}_{\mathrm{e}} / \mathrm{n}_{\mathrm{e}}^{3}=$ constant. The electron fluid density, $\mathrm{n}_{\mathrm{e}}$, can be expressed as a zero order background density, $\mathrm{n}_{\mathrm{O}}$, and a first order perturbation, $\mathrm{n}_{\mathrm{epw}}$, representing the Langmuir wave fluctuation present in the plasma. The velocity components can also be expressed as a zero order velocity, $\mathrm{u}_{\mathrm{o}}=\mathrm{qA}_{\mathrm{o}} / \mathrm{m}_{\mathrm{e}} \mathrm{c}$, representing the interaction beam and two first order components $u_{s c a t t}=\mathrm{qA}_{\mathrm{scatt}} / \mathrm{m}_{\mathrm{e}} \mathrm{c}$ and $\mathrm{u}_{\mathrm{epw}}$ representing the scattered electromagnetic wave and the Langmuir wave, respectively. Keeping only first order components, equation 2.1 .9 can be expressed as

$$
\begin{aligned}
& \frac{\partial^{2} n_{e p w}}{\partial \mathrm{t}^{2}}+\frac{4 \pi \mathrm{e}^{2} n_{\mathrm{oe}} \mathrm{n}_{\mathrm{epw}}}{\mathrm{m}_{\mathrm{e}}}-3 v_{\mathrm{th}}^{2} \nabla^{2} \mathrm{n}_{\mathrm{epw}}= \\
& \mathrm{n}_{\mathrm{oe}} \mathrm{c}^{2} \nabla^{2}\left(\frac{\mathrm{q} \overrightarrow{\mathrm{A}}_{\mathrm{o}}}{\mathrm{m}_{\mathrm{e}} \mathrm{c}^{2}} \cdot \frac{\mathrm{q} \overrightarrow{\mathrm{A}}_{\mathrm{scatt}}}{\mathrm{m}_{\mathrm{e}} \mathrm{c}^{2}}\right)
\end{aligned}
$$




\section{Appendix 2.2}

\section{Rosenbluth model for parametric instabilities in inhomogeneous plasmas}

This appendix reviews the calculation of the overall gain for convective parametric instabilities in inhomogeneous plasmas[27]. Appendix 2.4 shows the substitutions required to transform equation 2.1.6 and 2.1.10 in Appendix 2.1 to the steady-state equations describing parametric instabilities in the case of a linear phase mismatch between the waves which are

$$
\begin{aligned}
& \left(v_{\mathrm{s}}+\mathrm{V}_{\mathrm{s}} \frac{\mathrm{d}}{\mathrm{dx}}\right) \Psi_{\mathrm{s}}=\gamma_{\mathrm{o}} \Psi_{\mathrm{e}} \exp \left(\frac{-\mathrm{i} \kappa^{\prime} \mathrm{x}^{2}}{2}\right) \text { and } \\
& \left(v_{\mathrm{e}}-\mathrm{V}_{\mathrm{e}} \frac{\mathrm{d}}{\mathrm{dx}}\right) \Psi_{\mathrm{e}}=\gamma_{\mathrm{o}} \Psi_{\mathrm{s}} \exp \left(\frac{\mathrm{i} \kappa^{\prime} x^{2}}{2}\right) .
\end{aligned}
$$

With the following substitutions

$$
\Psi_{\mathrm{s}}=\Phi_{\mathrm{s}} \exp \left(\frac{-\mathrm{i} \kappa^{\prime} \mathrm{x}^{2}}{4}\right) \text { and } \Psi_{\mathrm{e}}=\Phi_{\mathrm{e}} \exp \left(\frac{\mathrm{i} \kappa^{\prime} \mathrm{x}^{2}}{4}\right)
$$


the previous equations may be put in a form which can be Fourier transformed easily. With the above substitutions, equations 2.2.1 and 2.2.2 become

$$
\begin{aligned}
& v_{\mathrm{s}} \Phi_{\mathrm{s}}+\mathrm{V}_{\mathrm{s}} \frac{\mathrm{d} \Phi_{\mathrm{s}}}{\mathrm{dx}}-\frac{\mathrm{i} \kappa^{\prime} \mathrm{x}}{2} \mathrm{~V}_{\mathrm{s}} \Phi_{\mathrm{s}}=\gamma_{\mathrm{o}} \Phi_{\mathrm{e}} \text { and } \\
& v_{\mathrm{e}} \Phi_{\mathrm{e}}-\mathrm{V}_{\mathrm{e}} \frac{\mathrm{d} \Phi_{\mathrm{e}}}{\mathrm{dx}}-\frac{\mathrm{i} \kappa^{\prime} \mathrm{x}}{2} \mathrm{~V}_{\mathrm{e}} \Phi_{\mathrm{e}}=\gamma_{\mathrm{o}} \Phi_{\mathrm{s}}
\end{aligned}
$$

These equations can then be Fourier transformed into the following equations

$$
\begin{aligned}
& {\left[v_{\mathrm{s}}+\mathrm{ikV} \mathrm{V}_{\mathrm{s}}+\frac{\kappa^{\prime} \mathrm{V}_{\mathrm{s}}}{2} \frac{\mathrm{d}}{\mathrm{dk}}\right] \Phi_{\mathrm{s}}(\mathrm{k})=\gamma_{\mathrm{o}} \Phi_{\mathrm{e}}(\mathrm{k}) \text { and }} \\
& {\left[v_{\mathrm{e}}-\mathrm{ikV} \mathrm{V}_{\mathrm{e}}+\frac{\kappa^{\prime} \mathrm{V}_{\mathrm{e}}}{2} \frac{\mathrm{d}}{\mathrm{dk}}\right] \Phi_{\mathrm{e}}(\mathrm{k})=\gamma_{\mathrm{o}} \Phi_{\mathrm{s}}(\mathrm{k})}
\end{aligned}
$$

These two equations can then be combined into a single equation describing the gain for the coupled modes participating in the parametric instability. This equation is

$$
\begin{aligned}
& {\left[\frac{\kappa^{\prime 2}}{4} \frac{\mathrm{d}^{2}}{\mathrm{dk}^{2}}+\frac{\kappa^{\prime}}{2}\left(\frac{v_{\mathrm{e}}}{\mathrm{V}_{\mathrm{e}}}+\frac{v_{\mathrm{s}}}{\mathrm{V}_{\mathrm{s}}}\right) \frac{\mathrm{d}}{\mathrm{dk}}-\mathrm{i} \frac{\kappa^{\prime}}{2}+\mathrm{k}^{2}\right.} \\
& \left.-\frac{\gamma_{0}^{2}}{\mathrm{~V}_{\mathrm{s}} \mathrm{V}_{\mathrm{e}}}+\frac{v_{\mathrm{e}} v_{\mathrm{s}}}{\mathrm{V}_{\mathrm{s}} \mathrm{V}_{\mathrm{e}}}+\mathrm{ik}\left(\frac{v_{\mathrm{e}}}{\mathrm{V}_{\mathrm{e}}}-\frac{v_{\mathrm{s}}}{\mathrm{V}_{\mathrm{s}}}\right)\right] \Phi_{\mathrm{e}}(\mathrm{k})=0 .
\end{aligned}
$$

Neglecting damping on the daughter waves, $v_{\mathrm{e}}=v_{\mathrm{s}}=0$, this equation reduces to 


$$
\frac{\mathrm{d}^{2} \Phi_{\mathrm{e}}(\mathrm{k})}{\mathrm{dk}^{2}}+\frac{4}{\kappa^{\prime 2}}\left[-\mathrm{i} \frac{\kappa^{\prime}}{2}+\mathrm{k}^{2}-\frac{\gamma_{0}^{2}}{\mathrm{~V}_{\mathrm{s}} \mathrm{V}_{\mathrm{e}}}\right] \Phi_{\mathrm{e}}(\mathrm{k})=0
$$

This is then solved by the WKB method[27] integrating between the zeros

$$
\Phi_{\mathrm{e}}(\mathrm{k}) \propto \exp \left\{\frac{2 \gamma_{0}^{2}}{\mathrm{~V}_{\mathrm{s}} \mathrm{V}_{\mathrm{e}} \kappa^{\prime}} \int_{-1}^{1} \sqrt{1-\mathrm{j}^{2}} \mathrm{dj}\right\} .
$$

The amplitude gain, assuming a linear phase mismatch, for the coupled mode described by the above equations is then given by

$$
\Phi_{\mathrm{e}}(\mathrm{k}) \propto \exp \left\{\frac{\pi \gamma_{0}^{2}}{\mathrm{~V}_{\mathrm{s}} \mathrm{V}_{\mathrm{e}} \kappa^{\prime}}\right\}
$$

This approach has also been used to calculate the gain at the top of a parabolic profile in which the wavenumber mismatch does not contain a nonzero derivative in the wavenumber mismatch. This is the case for stimulated Raman forward scatter on the top of a parabolic profile. The equations describe forward stimulated Raman scattering from the top of a parabolic density profile. The inhomogeneity in the light wave equation has been ignored in comparison with the inhomogeneity effects in the Langmuir wave equation so that the resulting equations,

$$
\left(v_{\mathrm{s}}+\mathrm{V}_{\mathrm{s}} \frac{\mathrm{d}}{\mathrm{dx}}\right) \Psi_{\mathrm{s}}=\gamma_{\mathrm{o}} \Psi_{\mathrm{e}} \text { and }
$$




$$
\left(v_{e}+\frac{i \omega_{p e}^{2} x^{2}}{2 \omega_{e} L_{n}^{2}}+V_{e} \frac{d}{d x}\right) \Psi_{e}=\gamma_{o} \Psi_{s}
$$

can be Fourier transformed into a single second-order differential equation which can be solved using WKB theory. The above two equations can then be Fourier transformed into the following equations

$$
\begin{aligned}
& {\left[v_{s}+i k V_{s}\right] \Phi_{s}(k)=\gamma_{o} \Phi_{e}(k) \text { and }} \\
& {\left[v_{e}+i k V_{e}+\frac{i \omega_{p e}^{2}}{2 \omega_{e} L_{n}^{2}} \frac{d^{2}}{d k^{2}}\right] \Phi_{e}(k)=\gamma_{o} \Phi_{s}(k)}
\end{aligned}
$$

These two equations can then be combined into a single equation describing the gain for the coupled modes participating in the parametric instability. This equation is

$$
\left[\frac{\mathrm{d}^{2}}{\mathrm{dk}^{2}}-\frac{1}{\kappa^{\prime \prime}}\left\{\frac{-\mathrm{k}^{2}+\mathrm{ik}\left(\frac{v_{\mathrm{e}}}{\mathrm{V}_{\mathrm{e}}}+\frac{v_{\mathrm{s}}}{\mathrm{V}_{\mathrm{s}}}\right)+\frac{v_{\mathrm{e}} v_{\mathrm{s}}-\gamma_{0}^{2}}{\mathrm{~V}_{\mathrm{s}} \mathrm{V}_{\mathrm{e}}}}{\left(\mathrm{i} \frac{v_{\mathrm{s}}}{\mathrm{V}_{\mathrm{s}}}-\mathrm{k}\right)}\right\} \Phi_{\mathrm{e}}(\mathrm{k})=0 .\right.
$$

Neglecting damping on the daughter waves, $v_{\mathrm{e}}=v_{\mathrm{S}}=0$, this equation reduces down to

$$
\left[\frac{\mathrm{d}^{2}}{\mathrm{dk}^{2}}-\frac{1}{\mathrm{k}^{\prime \prime}}\left(\mathrm{k}+\frac{\gamma_{0}^{2}}{\mathrm{kV}_{\mathrm{s}} \mathrm{V}_{\mathrm{e}}}\right)\right] \Phi_{\mathrm{e}}(\mathrm{k})=0
$$


This is then solved by the WKB method integrating between the zeros

$$
\Phi_{\mathrm{e}}(\mathrm{k}) \propto \exp \left\{\frac{4 \gamma_{\mathrm{o}}^{1.5}}{\left(\mathrm{~V}_{\mathrm{s}} \mathrm{V}_{\mathrm{e}}\right)^{0.75} \sqrt{\mathrm{K}^{\prime \prime}}} \int_{0}^{1} \sqrt{1-\mathrm{j}^{4}} \mathrm{dj}\right\}
$$

The amplitude gain, assuming a linear phase mismatch, for the coupled mode described by the above equations is then given by[29]

$$
\Phi_{\mathrm{e}}(\mathrm{k}) \propto \exp \left\{\frac{3.496 \gamma_{\circ}^{1.5}}{\left(\mathrm{~V}_{\mathrm{s}} \mathrm{V}_{\mathrm{e}}\right)^{0.75} \sqrt{\kappa^{\prime \prime}}}\right\}
$$





\section{Appendix 2.3}

\section{K-space resonance width for steady state}

The k-space resonance width is determined by Fourier transforming the coupled homogeneous equations describing parametric instabilities

$$
\begin{aligned}
& v_{\text {epw }} \Psi_{\text {epw }}+V_{\text {gepw }} \frac{d \Psi_{\text {epw }}}{d x}=\gamma_{1} \Psi_{\mathrm{s}}^{*}+\delta(x) \text { and } \\
& v_{s} \Psi_{s}^{*}+V_{g s} \frac{d \Psi_{s}^{*}}{d x}=\gamma_{2} \Psi_{\text {epw }} .
\end{aligned}
$$

The Fourier transformed amplitude is given by

$$
\Psi_{\text {epw }}(\mathrm{k})=\frac{\frac{v_{\mathrm{s}}}{\mathrm{V}_{\mathrm{gs}}}+\mathrm{ik}}{\mathrm{V}_{\mathrm{gepw}}\left(-\mathrm{k}^{2}+\mathrm{ik}\left(\frac{v_{\mathrm{s}}}{\mathrm{V}_{\mathrm{gs}}}+\frac{v_{\mathrm{epw}}}{\mathrm{V}_{\mathrm{gepw}}}\right)+\frac{-\gamma_{o}^{2}+v_{\mathrm{s}} v_{\mathrm{epw}}}{\mathrm{V}_{\mathrm{gepw}} \mathrm{V}_{\mathrm{gs}}}\right)}
$$

The square of the absolute magnitude of the Fourier transformed amplitude is then 


$$
\begin{aligned}
& \mathrm{g}(\mathrm{k})= \Psi_{\text {epw }}(\mathrm{k}) \Psi_{\text {epw }}^{*}(\mathrm{k})= \\
& \frac{\left(\frac{v_{\mathrm{s}}}{\mathrm{V}_{\mathrm{gs}}}\right)^{2}+\mathrm{k}^{2}}{\mathrm{~V}_{\text {gepw }}^{2}\left(\left(\mathrm{k}^{2}+\frac{\gamma_{0}^{2}-v_{\mathrm{s}} v_{\text {epw }}}{\mathrm{V}_{\text {gepw }} \mathrm{V}_{\mathrm{gs}}}\right)^{2}+\mathrm{k}^{2}\left(\frac{v_{\mathrm{s}}}{\mathrm{V}_{\mathrm{gs}}}+\frac{v_{\text {epw }}}{\mathrm{V}_{\text {gepw }}}\right)^{2}\right)}
\end{aligned}
$$

Provided $v_{\mathrm{s}} \mathrm{V}_{\mathrm{gs}}^{-1} \ll \sqrt{\gamma_{0}^{2}-v_{\mathrm{s}} v_{\mathrm{epw}}}\left(\mathrm{V}_{\mathrm{gepw}} \mathrm{V}_{\mathrm{gs}}\right)^{-0.5}, \mathrm{~g}(\mathrm{k})$ has it's maximum value when $\mathrm{k}=\sqrt{\gamma_{0}^{2}-v_{\mathrm{s}} v_{\text {epw }}}\left(\mathrm{V}_{\mathrm{gepw}} \mathrm{V}_{\mathrm{gs}}\right)^{-0.5}$ at which point $\mathrm{g}(\mathrm{k})$ has the value

$$
\mathrm{g}(\mathrm{k})=\Psi_{\text {epw }}(\mathrm{k}) \Psi_{\text {epw }}^{*}(\mathrm{k})=\frac{1}{\mathrm{~V}_{\mathrm{gepw}}^{2}\left(4\left(\frac{\gamma_{o}^{2}-v_{\mathrm{s}} v_{\text {epw }}}{\mathrm{V}_{\text {gepw }} \mathrm{V}_{\mathrm{gs}}}\right)+\left(\frac{v_{\mathrm{s}}}{\mathrm{V}_{\mathrm{gs}}}+\frac{v_{\text {epw }}}{\mathrm{V}_{\mathrm{gepw}}}\right)^{2}\right)}
$$

The k-space resonance width is now found by looking at the full width in $k$ space at half maximum of $g(k)$ given in 2.3.5. This $k$-space resonance width is then

$$
\begin{aligned}
& \Delta \mathrm{k} \approx\left\{\frac{3\left(\gamma_{0}^{2}-v_{\mathrm{s}} v_{\mathrm{epw}}\right)}{\mathrm{V}_{\mathrm{gepw}} \mathrm{V}_{\mathrm{gs}}}+\frac{1}{2}\left(\frac{v_{\mathrm{s}}}{\mathrm{V}_{\mathrm{gs}}}+\frac{v_{\mathrm{epw}}}{\mathrm{v}_{\mathrm{gepw}}}\right)^{2}+\right. \\
& \left.\sqrt{\frac{1}{4}\left[\frac{6\left(\gamma_{0}^{2}-v_{\mathrm{s}} v_{\text {epw }}\right)}{\mathrm{V}_{\mathrm{gepw}} \mathrm{V}_{\mathrm{gs}}}+\left(\frac{v_{\mathrm{s}}}{\mathrm{v}_{\mathrm{gs}}}+\frac{v_{\text {epw }}}{\mathrm{V}_{\text {gepw }}}\right)^{2}\right]^{2}-\left(\frac{\gamma_{0}^{2}-v_{\mathrm{s}} v_{\text {epw }}}{\mathrm{v}_{\mathrm{gepw}} \mathrm{V}_{\mathrm{gs}}}\right)^{2}}\right\}^{0.5} .
\end{aligned}
$$


With no damping $\Delta \mathrm{k} \propto \gamma_{\mathrm{o}}\left(\mathrm{V}_{\mathrm{epw}} \mathrm{V}_{\mathrm{s}}\right)^{-0.5}$ and in the strong damping limit where $v_{\text {epw }} \gg \gamma_{0} \mathrm{v}_{\text {gepw }}^{0.5} \mathrm{v}_{\mathrm{gs}}^{-0.5}, \Delta \mathrm{k} \propto v_{\mathrm{epw}} \mathrm{V}_{\mathrm{gepw}}^{-1}$.

The length over which the parametric instability can grow is determined by plasma inhomogeneities which dictate the length over which the waves become detuned. The wavenumber mismatch between the waves participating in the parametric process can be Taylor expanded about the perfect-phase-matching-point, $x_{0}$, to see how the how the phase changes with distance. Using this prescription, the wavenumber mismatch is given by

$$
\begin{aligned}
& \Delta k(x)=\left[k_{o}(x)-k_{s}(x)-k_{e}(x)\right] \approx \\
& \Delta k\left(x_{0}\right)+\left.\left(x-x_{0}\right) k^{\prime}\right|_{x_{o}}+\left.0.5\left(x-x_{0}\right)^{2} k^{\prime \prime}\right|_{x_{o}}{ }^{\prime}
\end{aligned}
$$

where it is assumed that at the perfect-phase-mismatch-point, $x_{0}, \Delta \mathbf{k}\left(x_{0}\right)=0$.

The interaction length, $x-x_{0}$, is found by equating the wavenumber resonance width to the Taylor expanded wavenumber mismatch, $\left.\gamma_{\mathrm{o}}\left(\mathrm{V}_{\mathrm{gepw}} \mathrm{V}_{\mathrm{gs}}\right)^{-0.5} \propto\left(\mathrm{x}-\mathrm{x}_{\mathrm{o}}\right) \mathrm{K}^{\prime}\right|_{\mathrm{x}_{\mathrm{o}}}+\left.0.5\left(\mathrm{x}-\mathrm{x}_{\mathrm{o}}\right)^{2} \mathrm{~K}^{\prime \prime}\right|_{\mathrm{x}_{\mathrm{o}}}$. In the case of a linear mismatch, neglecting damping, $\kappa^{\prime}>>\kappa^{\prime \prime}$ and $\mathrm{L}_{\text {lin }}=\left(x-x_{0}\right) \propto \gamma_{0} \kappa^{\prime-1}\left(V_{\text {gepw }} V_{g s}\right)^{-0.5}$. However, in the case where $\kappa^{\prime}=0$, the interaction distance is given by $\mathrm{L}_{\mathrm{par}}=\left(\mathrm{x}-\mathrm{x}_{\mathrm{o}}\right) \propto \gamma_{0}^{0.5} \kappa^{\prime,-0.5}\left(\mathrm{~V}_{\mathrm{gepw}} \mathrm{V}_{\mathrm{gs}}\right)^{-0.25}$ in the undamped case. In the strong damping limit, $v_{\text {epw }} \gg \gamma_{0} \mathrm{~V}_{\mathrm{gepw}}^{0.5} \mathrm{~V}_{\mathrm{gs}}^{-0.5}$, the interaction distance in the case of a linear phase mismatch is given by $\mathrm{L}_{\text {lin }} \propto v_{\text {epw }} \mathrm{V}_{\text {gepw }}^{-1} \kappa^{-1}$ and in the case of a parabolic phase mismatch is given by $\mathrm{L}_{\text {par }} \propto v_{\text {epw }}^{0.5} \mathrm{~V}_{\text {gepw }}^{-0.5} \kappa^{\prime \prime-0.5}$. 
The homogeneous solution to equation 2.1 and 2.2 is given by $\Psi_{\text {epw }} \propto \exp \left[x\left\{-0.5\left(\frac{v_{\text {epw }}}{V_{\text {gepw }}}+\frac{v_{\mathrm{s}}}{V_{\text {gs }}}\right)+\sqrt{0.25\left(\frac{v_{\text {epw }}}{V_{\text {gepw }}}+\frac{v_{\mathrm{s}}}{V_{\text {gs }}}\right)^{2}+\frac{\gamma_{\mathrm{o}}^{2}-v_{\text {epw }} v_{\mathrm{s}}}{\mathrm{V}_{\mathrm{gs}} \mathrm{V}_{\mathrm{gepw}}}}\right\}\right]$

Neglecting damping, the homogeneous amplitude is reduced to $\Psi_{\mathrm{s}} \alpha \exp \left[\gamma_{\mathrm{o}} \mathrm{x}\left(\mathrm{V}_{\mathrm{gepw}} \mathrm{V}_{\mathrm{gs}}\right)^{-0.5}\right]$. In the strong damping limit, $v_{\text {epw }} \gg \gamma_{0} \mathrm{v}_{\text {gepw }}^{0.5} \mathrm{v}_{\mathrm{gs}}^{-0.5}$, the homogeneous amplitude is given by $\Psi_{\text {epw }} \propto \exp \left[x \gamma_{0}^{2} v_{\mathrm{epw}}^{-1} \mathrm{~V}_{\mathrm{gs}}^{-1}\right]$. The parameter dependence of the inhomogeneous gain is found by inserting the interaction length into the expression for the homogeneous gain above. The parameter dependence for the gain in a plasma with a linear phase mismatch neglecting damping is therefore given by $\mathrm{G}_{\mathrm{srs}} \propto \gamma_{\mathrm{o}}^{2}\left(\mathrm{~V}_{\mathrm{gs}} \mathrm{V}_{\mathrm{gepw}} \kappa^{\prime}\right)^{-1}$. As shown in Appendix $2.2, \pi$ is the correct numerical coefficient giving the expression for the amplitude gain with a linear phase mismatch between the waves as $\Psi_{\text {epw }} \propto \exp \left[\pi \gamma_{0}^{2}\left(\mathrm{~V}_{\mathrm{gs}} \mathrm{V}_{\mathrm{gepw}} \kappa^{\prime}\right)^{-1}\right]$. In the strong damping limit, the parameter dependence for the gain in a plasma with a linear phase mismatch is also given by $G_{\text {srs }} \propto \gamma_{0}^{2}\left(V_{\text {gs }} V_{\text {gepw }} \kappa^{\prime}\right)^{-1}$ which has the same parameter dependence as the undamped case. The parameter dependence for the gain in a plasma with a quadratic phase mismatch neglecting damping is given by $\mathrm{G}_{\mathrm{srs}} \propto \gamma_{\mathrm{o}}^{1.5}\left(\mathrm{~V}_{\mathrm{gs}} \mathrm{V}_{\mathrm{gepw}}\right)^{-0.75} \kappa^{\prime \prime}-0.5$. As shown in Appendix 2.2, 3.496 is the correct numerical coefficient giving the expression for the amplitude gain with a quadratic phase mismatch between the waves as $\Psi_{\text {epw }} \propto \exp \left[3.496 \gamma_{0}^{1.5}\left(\mathrm{~V}_{\mathrm{gs}} \mathrm{V}_{\text {gepw }}\right)^{-0.75} \kappa^{\prime \prime-0.5}\right]$. In the strong damping limit, the parameter dependence for the gain in a plasma with a quadratic phase 
APPENDIX 2.3 K-SPACE RESONANCE WIDTH FOR STEADY . . . . . 196

mismatch is given by $\mathrm{G}_{\mathrm{srs}} \propto \gamma_{\mathrm{o}}^{2}\left(v_{\mathrm{epw}} \mathrm{V}_{\mathrm{gepw}} \kappa^{\prime \prime}\right)^{-0.5} \mathrm{~V}_{\mathrm{gs}}^{-1}$ with 4.45 giving the correct numerical coefficient for the amplitude gain. 


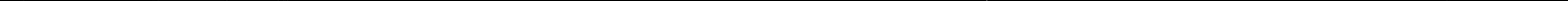




\section{Appendix 2.4}

\section{Numerical solution of Rosenbluth equations}

This appendix details the numerical calculation for parametric instabilities driven from a linear phase mismatch. This calculation solves two first-order coupled partial differential equations by the method of characteristics which has been used previously to investigate these equations[112]. With very slight modification, the code given below can treat forward and backward scattering, quadratic phase mismatches, background density perturbations, convective or absolute instabilities, etc..

From Appendix 2.1, the equations describing the growth of the daughter waves participating in stimulated Raman scattering are

$$
\begin{aligned}
& \left(\frac{\partial^{2}}{\partial t^{2}}+\omega_{p e}^{2}-c^{2} \nabla^{2}\right) \frac{q \vec{A}_{s c a t t}}{m_{e} c^{2}}=-\omega_{p e}^{2} \frac{n_{e p w}}{n_{o e}} \frac{q \vec{A}_{p u m p}}{m_{e} c^{2}} \text { and } \\
& \left(\frac{\partial^{2}}{\partial t^{2}}+\omega_{p e}^{2}-3 v_{t h}^{2} \nabla^{2}\right) \frac{n_{e p w}}{n_{o e}}=c^{2} \nabla^{2}\left(\frac{q \vec{A}_{s c a t t}}{m_{e} c^{2}} \cdot \frac{q \vec{A}_{p u m p}}{m_{e} c^{2}}\right) .
\end{aligned}
$$


These equations can be further simplified by writing the vector potentials and the Langmuir wave fluctuation as a slowly varying component multiplied by a rapid oscillation, or more precisely $\frac{\mathrm{qA}_{\alpha}}{\mathrm{m}_{\mathrm{e}} \mathrm{c}^{2}}=\frac{1}{2} \Psi_{\alpha}(\mathrm{x}, \mathrm{t}) \exp \left(\mathrm{i} \int \overrightarrow{\mathrm{k}}_{\alpha} \cdot \mathrm{d} \overrightarrow{\mathrm{x}}-\mathrm{i} \omega \mathrm{t}\right)+$ c.c.. $\quad$ The assumption that $\frac{\partial \Psi_{\alpha}(x, t)}{\partial x} \ll k \Psi_{\alpha}(x, t)$ and $\frac{\partial \Psi_{\alpha}(x, t)}{\partial t} \ll \omega \Psi_{\alpha}(x, t)$ reduces the above equations to the following first-order partial differential equations

$$
\begin{aligned}
& \left(\frac{\partial}{\partial \mathrm{t}}+v_{\text {scatt }}+\overrightarrow{\mathrm{V}}_{\text {scatt }} \cdot \frac{\partial}{\partial \vec{x}}\right) \Psi_{\text {scatt }}^{*}= \\
& \frac{-\omega_{\text {pe }}^{2}}{4 i \omega_{\text {scatt }}} \Psi_{\text {epw }} \Psi_{\text {pump }} \exp \left(\mathrm{i} \int \Delta \mathrm{k}(\mathrm{x}) \mathrm{dx}\right) \text { and } \\
& \left(\frac{\partial}{\partial \mathrm{t}}+v_{\mathrm{epw}} \pm \overrightarrow{\mathrm{V}}_{\mathrm{epw}} \cdot \frac{\partial}{\partial \overrightarrow{\mathrm{x}}}\right) \Psi_{\text {epw }}= \\
& \frac{-\mathrm{c}^{2} \mathrm{k}_{\mathrm{epw}}^{2}}{4 \mathrm{i} \omega_{\text {epw }}} \Psi_{\text {scatt }}^{*} \Psi_{\text {pump }} \exp \left(-\mathrm{i} \int \Delta \mathrm{k}(\mathrm{x}) \mathrm{dx}\right)
\end{aligned}
$$

where $v_{\text {epw }}$ and $v_{\text {scatt }}$ are the damping of the Langmuir wave and the scattered electromagnetic wave respectively. The Taylor expanded phase mismatch for a linear profile is $\Delta k(x)=k_{\text {pump }}-k_{\text {epw }} \mp k_{\text {scatt }} \approx \kappa^{\prime}\left(x_{0}\right) x$. Making the further substitutions $\Psi_{\text {epw }}=\phi_{\text {epw }}(-\mathrm{i}) \sqrt{c^{2} k_{\text {epw }}^{2} / 4 \omega_{\text {epw }}}$ and $\Psi_{\text {scatt }}^{*}=\phi_{\text {scatt }} \sqrt{\omega_{\text {pe }}^{2} / 4 \omega_{\text {scatt }}}$ allows equations 2.4 .3 and 2.4.4 to be written in the form

$$
\left(\frac{\partial}{\partial \mathrm{t}}+v_{\text {scatt }}+\mathrm{V}_{\text {scatt }} \frac{\partial}{\partial \mathrm{x}}\right) \phi_{\text {scatt }}=\gamma_{\mathrm{o}} \phi_{\mathrm{epw}} \exp \left(\mathrm{i} \frac{\kappa^{\prime} x^{2}}{2}\right) \text { and }
$$




$$
\left(\frac{\partial}{\partial t}+v_{\text {epw }} \pm \mathrm{V}_{\mathrm{epw}} \frac{\partial}{\partial \mathrm{x}}\right) \phi_{\mathrm{epw}}=\gamma_{\mathrm{o}} \phi_{\mathrm{scatt}} \exp \left(-\mathrm{i} \frac{\kappa^{\prime} x^{2}}{2}\right)
$$

where $\gamma_{0}$ is the homogeneous growth rate for stimulated Raman scattering defined as $\gamma_{\mathrm{o}}=\mathrm{k}_{\mathrm{epw}} \mathrm{c} \Psi_{\text {pump }} \omega_{\mathrm{pe}} /\left(4 \sqrt{\omega_{\mathrm{epw}} \omega_{\text {scatt }}}\right)$. The above equations are then normalized with the variable substitutions $\tau=\gamma_{0} t$ and $\chi=\gamma_{\mathrm{o}} \mathrm{x} / \sqrt{\mathrm{V}_{\text {epw }} \mathrm{V}_{\text {scatt }}}$. These variable substitutions allow equations 2.4 .5 and 2.4.6 to be written as

$$
\begin{aligned}
& \left(\frac{\partial}{\partial \tau}+\sqrt{\frac{\mathrm{V}_{\text {scatt }}}{V_{\text {epw }}}} \frac{\partial}{\partial \chi}\right) \phi_{\text {scatt }}= \\
& -\frac{v_{\text {scatt }}}{\gamma_{0}} \phi_{\text {scatt }}+\phi_{\text {epw }} \exp \left(\frac{i}{2} \frac{\kappa^{\prime} V_{\text {epw }} \mathrm{V}_{\text {scatt }}}{\gamma_{o}^{2}} \chi^{2}\right) \text { and } \\
& \left(\frac{\partial}{\partial \tau} \pm \sqrt{\frac{V_{\text {epw }}}{V_{\text {scatt }}}} \frac{\partial}{\partial \chi}\right) \phi_{\text {epw }}= \\
& -\frac{v_{\text {epw }}}{\gamma_{\mathrm{o}}} \phi_{\text {epw }}+\phi_{\text {scatt }} \exp \left(\frac{-i}{2} \frac{\kappa^{\top} \mathrm{V}_{\text {epw }} \mathrm{V}_{\text {scatt }}}{\gamma_{o}^{2}} \chi^{2}\right)
\end{aligned}
$$

The method of characteristics can be used to numerically solve equations 2.4.7 and 2.4 .8 by integrating along the characteristic. The substitutions required for the method of characteristics are $\xi=\chi+\tau \sqrt{\mathrm{V}_{\text {epw }} / \mathrm{V}_{\text {scatt }}}$ and $\eta=\chi-\tau \sqrt{\mathrm{V}_{\text {scatt }} / \mathrm{V}_{\text {epw }}}$. Equations 2.4.7 and 2.4.8 can now be written as 


$$
\begin{aligned}
& \frac{\partial \phi_{\text {epw }}}{\partial \xi}=\frac{1}{\left(\sqrt{\frac{V_{\text {scatt }}}{V_{\text {epw }}}}+\sqrt{\frac{V_{\text {epw }}}{V_{\text {scatt }}}}\right)} \times \\
& \left\{-\frac{v_{\text {epw }}}{\gamma_{o}} \phi_{\text {epw }}+\phi_{\text {scatt }} \exp \left(\frac{-i}{2} \frac{\kappa^{\prime} V_{\text {epw }} \mathrm{V}_{\text {scatt }}}{\gamma_{o}^{2}} \chi^{2}\right)\right\} \text { and } \\
& \frac{\partial \phi_{\text {scatt }}}{\partial \eta}=\frac{ \pm 1}{\left(\sqrt{\frac{V_{\text {scatt }}}{V_{\text {epw }}}}+\sqrt{\frac{V_{\text {epw }}}{V_{\text {scatt }}}}\right)} \times \\
& \left\{-\frac{v_{\text {scatt }}}{\gamma_{\text {o }}} \phi_{\text {scatt }}+\phi_{\text {epw }} \exp \left(\frac{i}{2} \frac{\kappa^{\prime} V_{\text {epw }} V_{\text {scatt }}}{\gamma_{o}^{2}} \chi^{2}\right)\right\}
\end{aligned}
$$

Figure A.2.4.1 shows a graphical representation of the method of characteristics. The spatial and temporal step used in the numerical evaluation of equation 2.4 .9 and 2.4.10 are chosen to fall along the intersection of the characteristics as shown in figure A.2.4.1. The relationship between the spatial step, $\Delta \chi$, and the temporal step, $\Delta \tau$, is given by $\Delta \chi=\Delta \tau \sqrt{\mathrm{V}_{\mathrm{epw}} / \mathrm{V}_{\text {scatt }}}$. The relationship between the change in the characteristic variables is given by $\Delta \eta=-\Delta \xi$, and the relationship between the characteristic variable and the temporal time step is given by $\Delta \xi=\Delta \tau\left(\sqrt{\frac{V_{\text {scatt }}}{V_{\text {epw }}}}+\sqrt{\frac{V_{\text {epw }}}{V_{\text {scatt }}}}\right)$. The differenced equations used in the program are

$$
\begin{aligned}
& \phi_{\text {epw }}(\eta+\Delta \eta, \xi)=\phi_{\text {epw }}(\eta, \xi)+\Delta \tau * \\
& \left\{-\frac{v_{\text {epw }}}{\gamma_{\mathrm{o}}} \phi_{\text {epw }}(\eta, \xi)+\phi_{\text {scatt }}(\eta, \xi) \exp \left(\frac{-i}{2} \frac{\kappa V_{\text {epw }} V_{\text {scatt }}}{\gamma_{o}^{2}} \chi^{2}\right)\right\} \text { and }
\end{aligned}
$$




$$
\begin{aligned}
& \phi_{\text {scatt }}(\eta, \xi+\Delta \xi)=\phi_{\text {scatt }}(\eta, \xi)+\Delta \tau * \\
& \left\{-\frac{v_{\text {scatt }}}{\gamma_{o}} \phi_{\text {scatt }}(\eta, \xi)+\phi_{\text {epw }}(\eta, \xi) \exp \left(\frac{i}{2} \frac{\kappa^{\top} V_{\text {epw }} \mathrm{V}_{\text {scatt }}}{\gamma_{o}^{2}} \chi^{2}\right)\right\}
\end{aligned}
$$

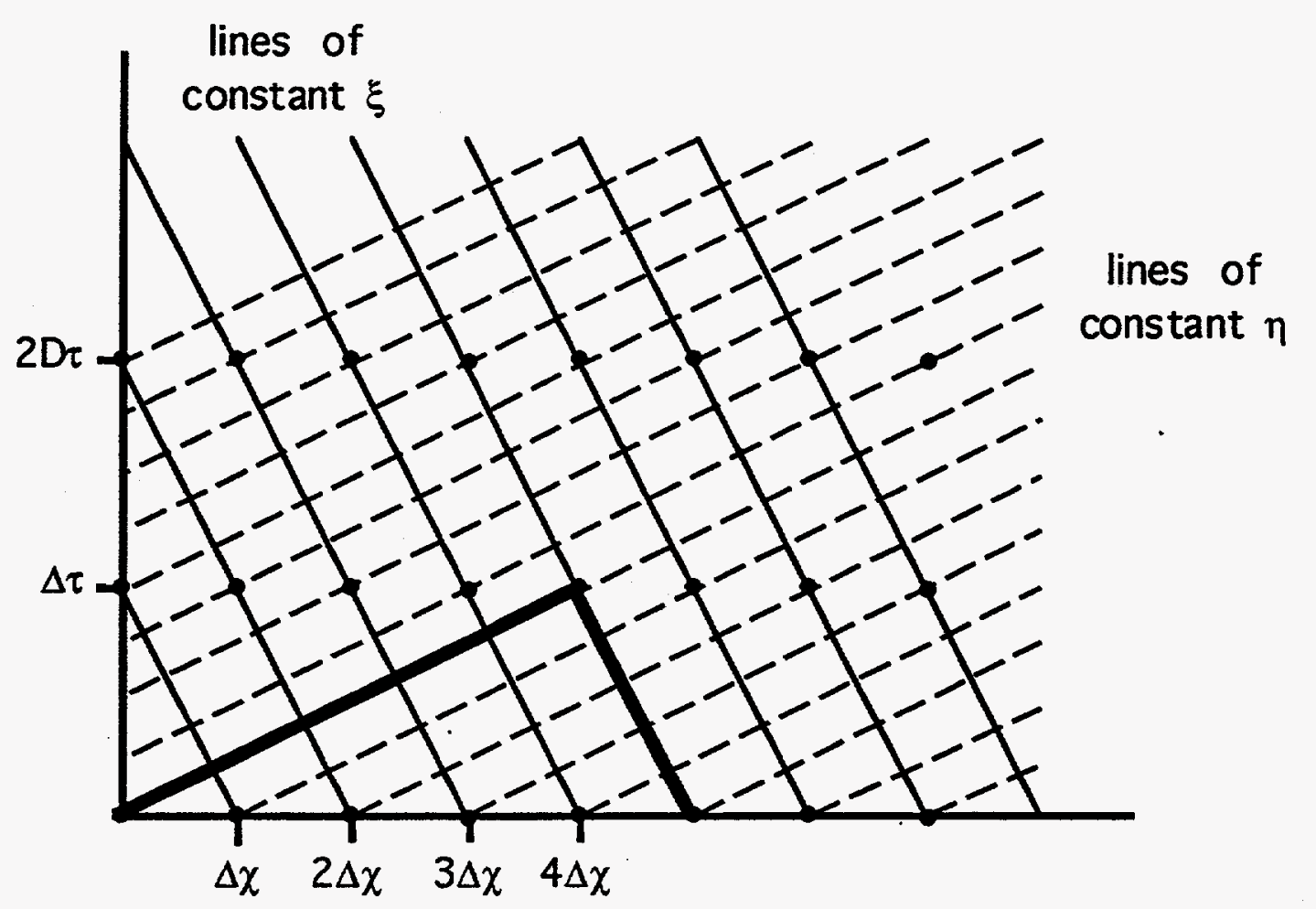

Figure A.2.4.1 Grid spacing used in the numerical program in the case of backscatter where the group velocity of the two waves are opposing.

The actual program follows:

C This program will look at the overall Rosenbluth gain 
$C$ for forward scattered daughter waves.

$C$ This version uses method of characteristics.

PARAMETER (NSTEP $=2000$ )

COMPLEX ScaNew(-NSTEP:NSTEP),ScaOld(-NSTEP:NSTEP), !EpwNew(-NSTEP:NSTEP),EpwOld(-NSTEP:NSTEP),i,

!Disp(-NSTEP:NSTEP),NuL,NuE,DTs,DTe, !ScaTime(0:NSTEP)

REAL Gamma, NuLight, NuEpw, Vratio, Time, VLight, !DT,DX, VEPW

INTEGER NITER, Vrat open(unit=13,file='Expdet0',status='unknown') close(unit $=13$,status $=$ 'delete') open(unit=13,file='Expdet0',status='unknown') open(unit=14,file='Expdet1',status='unknown') close (unit=14,status='delete') open(unit=14,file='Expdet1',status='unknown') open (unit $=15$,file $=$ 'Expdet2', status='unknown') close(unit $=15$,status='delete') open(unit=15,file='Expdet2',status='unknown') open(unit=16,file ='Expdet3', status='unknown') close (unit=16,status='delete') open(unit=16,file='Expdet3',status='unknown') open(unit=17, file='Expdet4', status='unknown') close (unit=17,status='delete') open(unit=17,file='Expdet4', status='unknown') open(unit=18,file='Exptime',status='unknown') close(unit=18,status='delete') open (unit=18,file='Exptime',status='unknown') $\mathrm{i}=\mathrm{CMPLX}(0 ., 1$.

10 PRINT *, 'Enter THE TOTAL NUMBER OF TIME STEPS (0 TO STOP)' 12 PRINT *, 'SUGGEST 1200.'

READ *,NITER IF (NITER .EQ. 0.) STOP

14 PRINT *, 'ENTER Gamma, NuLight, NuEpw, and Vratio'

16 PRINT *, 'SUGGEST 1.0, 0.0, 0.0, 1.0'

READ *, Gamma, NuLight, NuEpw, Vratio

IF (Gamma .EQ. 0.) STOP

VLight $=$ SQRT (Vratio)

$C$ forward scattering uses +VEpw

VEpw $=1.0 / \mathrm{SQRT}$ (Vratio)

$\mathrm{DT}=0.00125$

$\mathrm{DX}=\mathrm{DT} / \mathrm{SQRT}$ (Vratio)

Vrat $=$ Vratio 
$C$ setup initial wave amplitudes

DO 20 IX=-NSTEP,NSTEP

ScaOld $(\mathrm{IX})=$ CMPLX $(0.000,0$.

$\operatorname{ScaNew}(\mathrm{IX})=\operatorname{CMPLX}(0.000,0$.

EpwOId(IX) $=$ CMPLX $(0.000,0$.

$\operatorname{EpwNew}(\mathrm{IX})=\mathrm{CMPLX}(0.000,0$.

$\operatorname{Disp}(\mathrm{IX})=0.5^{*} \mathrm{i}^{*}\left(\operatorname{REAL}\left(\mathrm{DX}{ }^{*} \mathrm{IX}\right)\right)^{* *} 2.0 /$ Gamma

20 CONTINUE

DO 25 IM=-NSTEP,NSTEP

$C$ this ensures a smooth output

EpwOld(IM) $=$ CMPLX $(1,0$.

25 CONTINUE

$C$ end setup initial wave amplitudes

$C$ verify initial wave amplitudes

DO $30 \mathrm{IV}=-\mathrm{NSTEP}, \mathrm{NSTEP}$

C PRINT *,Epw=',EpwOld(IV),' PmpMag=',CABS(EpwOld(IV))

C PRINT *',Disp=',Disp(IV)

30 CONTINUE

$C$ end verify initial wave amplitudes

Time $=0$.

C time loop

$\mathrm{NuL}=\mathrm{CMPLX}\left(\mathrm{DT}^{*}\right.$ NuLight, 0.0$)$

$\mathrm{NuE}=\mathrm{CMPL} X\left(\mathrm{DT} \mathrm{T}^{*} \mathrm{NuEpw}, 0.0\right)$

$\mathrm{DTs}=\mathrm{CMPLX}(\mathrm{DT}, 0.0)$

$\mathrm{DTe}=\mathrm{CMPLX}(\mathrm{DT}, 0.0)$

C PRINT *, NuL,NuE,Dts,Dte

$C$ take care of boundary conditions

DO 35 IP=-NSTEP, -NSTEP+Vrat

$\operatorname{EpwNew}(\mathrm{IP})=\mathrm{CMPLX}(1.0,0.0)$

35 CONTINUE

$C$ end boundary conditions

DO 40 ITER=1, NITER

Time $=$ Time + DT

ScaTime $($ ITER $)=$ ScaOld $(0)$

$C$ fill out amplitude arrays at new time step

DO 50 IX=-NSTEP+Vrat, NSTEP

ScaNew(IX) $=$ ScaOld(IX-Vrat)-

1 ScaOld(IX-Vrat)*NuL+

! DTs*EpwOld(IX-Vrat) ${ }^{*}$ CEXP(

1 -Disp(IX-Vrat))

EpwNew(IX)=EpwOld(IX-1)-

! EpwOld(IX-1)*NuE+

! DTe*ScaOld(IX-1)*CEXP(Disp(IX-1)) 
50 CONTINUE

DO $60 \mathrm{JX}=-\mathrm{NSTEP}, \mathrm{NSTEP}$

$\mathrm{ScaOld}(\mathrm{JX})=\mathrm{ScaNew}(\mathrm{JX})$

EpwOld(JX) $=$ EpwNew (TX)

60 CONTINUE

IF (TTER.EQ. 3) THEN write $\left(13,{ }^{*}\right)$,'Mag. of As', ',','Mag. of Epw',,',',position'

DO $70 \mathrm{JX}=-$ NSTEP, NSTEP, 4 write $(13, *)$, CABS(ScaOld(JX)), ,',

$!$ CABS(EpwOld(JX)), ,',JX*DX

70 Continue close(unit=13)

Else IF (TTER.EQ.NITER/2) THEN write $\left(14,{ }^{*}\right)$, 'Mag. of As',,','Mag. of Epw',,',',position' DO 75 JX=-NSTEP, NSTEP, 4 write $(14, *), \mathrm{CABS}(\mathrm{ScaOld}(\mathrm{JX})),,$, ,

$!$ CABS(EpwOld(JX)), ', JJ $X^{* D X}$

75 Continue close(unit=14)

Else IF (ITER.EQ.2*NITER/3) THEN write $\left(15,{ }^{*}\right)$, Mag. of As',,, ,'Mag. of Epw',,',',position' DO 80 JX=-NSTEP, NSTEP, 4 write $\left(15,{ }^{*}\right)$, CABS(ScaOld(JX)), ,',

! CABS(EpwOld(TX)), ,',JX*DX

80 Continue close(unit=15)

Else IF (ITER.EQ. $5^{*}$ NITER/6) THEN write $\left(16,{ }^{*}\right)$, 'Mag. of As', ',','Mag. of Epw',,',',position' DO 85 JX=-NSTEP, NSTEP, 4 write $(16, *), \mathrm{CABS}(\mathrm{ScaOld}(\mathrm{JX})),,$,

! CABS(EpwOld(JX)), , ',JX*DX

85 Continue close(unit=16)

Else IF (ITER.EQ.NITER-1) THEN write $(17, *)$, 'Mag. of As', ',','Mag. of Epw',',','position' DO $90 \mathrm{JX}=-\mathrm{NSTEP}$, NSTEP, 4 write $\left(17,{ }^{*}\right), \mathrm{CABS}(\mathrm{ScaOld}(\mathrm{JX})),,$, ,

! CABS(EpwOld(JX)),;,jX*DX

90 Continue close (unit=17)

END IF

40 CONTINUE

write $\left(18,{ }^{*}\right)$, 'Mag. of As',,',',time' 
APPENDIX 2.4 NUMERICAL SOLUTION OF ROSENBLUTH . . . . . 205

DO 95 IL $=1$, NITER, 5

write $(18, *), \mathrm{CABS}(\mathrm{ScaTime}(\mathrm{IL})),,,, \mathrm{IL}{ }^{*} \mathrm{DT}$

95 CONTINUE

close(unit=18)

END 



\section{Appendix 2.5}

\section{Saturation of forward Raman scattering due to ponderomotive detuning}

This chapter details the derivation of the Langmuir wave's saturated amplitude due to ponderomotive detuning. This saturation mechanism was proposed as a saturation mechanism for stimulated Raman forward scattering and the derivation contained in this appendix follows the original paper [38]. The approximations made in this derivation, as well as their consequences, are discussed in chapter 2 section 2.7 .

The derivation begins with the equations describing the low frequency response of the ion and electrons due to the ponderomotive pressure of the high frequency waves. This equation can be derived from the momentum equation for the electrons and the continuity and momentum equations for the ions. Upon neglect of the electron inertial term in the electron momentum equation, the ion density perturbation, $\mathrm{n}_{\mathrm{i} 1}$, can be written in terms of the electron density perturbation, $\mathrm{n}_{\mathrm{el}}$, as 


$$
\mathrm{Zn}_{\mathrm{il}}=\mathrm{n}_{\mathrm{el}}-\left(\frac{\mathrm{v}_{\mathrm{th}}}{\omega_{\mathrm{pe}}}\right)^{2} \nabla^{2} \mathrm{n}_{\mathrm{el}}-\frac{\mathrm{n}_{\mathrm{oe}}}{2 \omega_{\mathrm{pe}}^{2}} \nabla^{2}\left(\mathrm{u}_{\mathrm{eh}}^{2}+\mathrm{u}_{20 \mathrm{~s}}^{2}\right),
$$

where $\mathbf{Z}$ is the charge state, $\mathfrak{u}_{\mathrm{eh}}$ is the oscillation velocity from the high frequency Langmuir wave, and $\mathrm{u}_{208}$ is the oscillation velocity from the high frequency electromagnetic daughter wave. The continuity and momentum equations for the ions can be combined to relate the ion density perturbation to the low frequency electron perturbation and the results combined with equation 2.5.1 to give the equation expressing the low frequency response of the electrons to the high frequency fields. This equation can be written as

$$
\left(\frac{\partial^{2}}{\partial t^{2}}-c_{s}^{2} \nabla^{2}\right) \frac{n_{e t}}{n_{o e}}=\frac{Z m_{e}}{2 M_{i}} \nabla^{2}\left(\left|u_{e h}\right|^{2}+\left|u_{2 o s}\right|^{2}\right),
$$

where $n_{e l} / n_{o e}$ is the amplitude of the low frequency response, $m_{e}$ is the mass of the electron, $\mathrm{M}_{\mathrm{i}}$ is the mass of the ion species, and $c_{\mathrm{S}}$ is the sound speed. Equation 2.5.2 is essentially the low frequency Zakharov equation. Making the quasi-static approximation, $\partial / \partial t<<c_{s} \partial / \partial x$, equation 2.5 .2 becomes

$$
\frac{\mathrm{n}_{\mathrm{el}}}{\mathrm{n}_{\mathrm{oe}}}=-\frac{1}{2}\left[\frac{\left|\mathrm{u}_{\mathrm{eh}}\right|^{2}}{\mathrm{v}_{\mathrm{th}}^{2}}+\frac{\left|\mathrm{u}_{2 \mathrm{os}}\right|^{2}}{\mathrm{v}_{\mathrm{th}}^{2}}\right],
$$

where 2.5.3 has been averaged over time removing high frequency components. The equation representing the amplitude of the Langmuir 
wave, $n_{\text {epw }} / \mathrm{n}_{\mathrm{oe}}$, participating in Raman scattering is derived in the same manner as shown in appendix 2.1 and is given by

$$
\left[\frac{\partial^{2}}{\partial t^{2}}+\omega_{p e}^{2}\left(1+\frac{n_{e l}}{n_{o e}}\right)-\frac{3 \kappa T_{e}}{m_{e}} \nabla^{2}\right] \frac{n_{e p w}}{n_{o e}}=c^{2} \nabla^{2}\left(\frac{q A_{s c a t t}}{m_{e} c^{2}} \cdot \frac{q A_{p u m p}}{m_{e} c^{2}}\right)
$$

The equation representing the scattered vector potential, $\mathrm{A}_{\text {scatt, }}$ is given by

$$
\left[\frac{\partial^{2}}{\partial \mathrm{t}^{2}}+\omega_{\mathrm{pe}}^{2}\left(1+\frac{\mathrm{n}_{\mathrm{el}}}{\mathrm{n}_{\mathrm{oe}}}\right)-c^{2} \nabla^{2}\right] \frac{\mathrm{qA}_{\mathrm{scatt}}}{\mathrm{m}_{\mathrm{e}} \mathrm{c}^{2}}=-\omega_{\mathrm{pe}}^{2} \frac{\mathrm{n}_{\mathrm{epw}}}{\mathrm{n}_{\mathrm{oe}}} \frac{\mathrm{qA} \mathrm{A}_{\mathrm{pump}}}{\mathrm{m}_{\mathrm{e}} \mathrm{c}^{2}}
$$

The low frequency response shown in equation 2.5 .3 can be inserted into equations 2.5.4 and 2.5.5 to produce the equations

$$
\begin{aligned}
& {\left[\frac{\partial^{2}}{\partial \mathrm{t}^{2}}+\omega_{\mathrm{pe}}^{2}\left\{1-\frac{1}{2}\left(\frac{1}{k^{2} \lambda_{\mathrm{oe}}^{2}}\left|\frac{\mathrm{n}_{\mathrm{epw}}}{\mathrm{n}_{\mathrm{oe}}}\right|^{2}+\frac{c^{2}}{v_{t h}^{2}}\left|\frac{\mathrm{qA} \mathrm{A}_{\text {scatt }}}{\mathrm{m}_{\mathrm{e}} \mathrm{c}^{2}}\right|^{2}\right)\right\}-\frac{3 \kappa \mathrm{T}_{\mathrm{e}}}{\mathrm{m}_{\mathrm{e}}} \nabla^{2}\right] \frac{\mathrm{n}_{\mathrm{epw}}}{\mathrm{n}_{\mathrm{oe}}}} \\
& =\mathrm{c}^{2} \nabla^{2}\left(\frac{\mathrm{qA}}{\mathrm{m}_{\mathrm{e}} \mathrm{c}^{2}} \cdot \frac{\mathrm{qA} \mathrm{A}_{\mathrm{pump}}}{\mathrm{m}_{\mathrm{e}} \mathrm{c}^{2}}\right) \text { and } \\
& {\left[\frac{\partial^{2}}{\partial \mathrm{t}^{2}}+\omega_{\mathrm{pe}}^{2}\left\{1-\frac{1}{2}\left(\frac{1}{k^{2} \lambda_{\mathrm{oe}}^{2}}\left|\frac{\mathrm{n}_{\mathrm{epw}}}{\mathrm{n}_{\mathrm{oe}}}\right|^{2}+\frac{c^{2}}{v_{t h}^{2}}\left|\frac{\mathrm{qA}_{\mathrm{scatt}}}{\mathrm{m}_{\mathrm{e}} \mathrm{c}^{2}}\right|^{2}\right)\right\}-c^{2} \nabla^{2}\right] \frac{\mathrm{qA}_{\text {scatt }}}{\mathrm{m}_{\mathrm{e}} \mathrm{c}^{2}}} \\
& =-\omega_{\mathrm{pe}}^{2} \frac{\mathrm{n}_{\mathrm{epw}}}{\mathrm{n}_{\mathrm{oe}}} \frac{\mathrm{qA} \mathrm{Aump}_{\mathrm{pump}}}{\mathrm{m}_{\mathrm{e}} \mathrm{c}^{2}}
\end{aligned}
$$


These equations are reduced by assuming that the Langmuir wave amplitude and the vector potentials can be written as a slowly varying component multiplied by a fast oscillation or

$$
\begin{aligned}
& \Psi_{\text {pump }}=\left(q A_{\text {pump }} / m_{e} c^{2}\right)=0.5 \Psi_{o}(x, t)\left[\exp \left(i k_{o} x-i \omega_{o} t\right)+c . c .\right], \\
& \Psi_{\text {scatt }}=\left(\mathrm{qA}_{\text {scatt }} / \mathrm{m}_{\mathrm{e}} \mathrm{c}^{2}\right)=0.5\left[\Psi_{\mathrm{s}}(\mathrm{x}, \mathrm{t}) \exp \left(\mathrm{ik_{s }} \mathrm{x}-\mathrm{i} \omega_{\mathrm{s}} \mathrm{t}\right)+\mathrm{c} . \mathrm{c} .\right] \text {, and } \\
& \Psi_{\text {epw }}=\left(n_{\text {epw }} / n_{\text {oe }}\right)=0.5\left[\Psi_{\text {epw }}(x, t) \exp \left(i k_{\text {epw }} x-i \omega_{\text {epw }} t\right)+c . c .\right] \text {. }
\end{aligned}
$$

When the equations in 2.5.7 are inserted into 2.5.6 and the dispersion relation for the waves are used, the equations can be written as

$$
\begin{aligned}
& -\frac{\omega_{\mathrm{pe}}^{2}}{8}\left(\frac{1}{k_{\mathrm{epw}}^{2} \lambda_{\mathrm{De}}^{2}}\left|\Psi_{\mathrm{epw}}\right|^{2}+\frac{c^{2}}{\mathrm{v}_{\mathrm{th}}^{2}}\left|\Psi_{\mathrm{s}}\right|^{2}\right) \Psi_{\mathrm{epw}}-\mathrm{i} 3 \mathrm{v}_{\mathrm{th}}^{2} \mathrm{k}_{\mathrm{epw}} \frac{\partial \Psi_{\mathrm{epw}}}{\partial \mathrm{x}} \\
& =\frac{-\mathrm{c}^{2} \mathrm{k}_{\mathrm{epw}}^{2}}{4} \Psi_{\mathrm{o}} \Psi_{\mathrm{s}}^{*} \text { and } \\
& -\frac{\omega_{\mathrm{pe}}^{2}}{8}\left(\frac{1}{k_{\mathrm{epw}}^{2} \lambda_{\mathrm{De}}^{2}}\left|\Psi_{\mathrm{epw}}\right|^{2}+\frac{\mathrm{c}^{2}}{\mathrm{v}_{\mathrm{th}}^{2}}\left|\Psi_{\mathrm{s}}\right|^{2}\right) \Psi_{\mathrm{s}}^{*}-\mathrm{ic}{ }^{2} \mathrm{k}_{\mathrm{s}} \frac{\partial \Psi_{\mathrm{s}}^{*}}{\partial \mathrm{x}} \\
& =\frac{-\omega_{\mathrm{pe}}^{2}}{4} \Psi_{0} \Psi_{\text {epw }} .
\end{aligned}
$$

The above two equations plus their complex conjugates represent the four equations which must be solved. These equations can be written as 


$$
\begin{aligned}
& \alpha \Psi_{\mathrm{epw}}-\mathrm{i} \beta \frac{\partial \Psi_{\mathrm{epw}}}{\partial \mathrm{x}}=\gamma_{1} \Psi_{\mathrm{s}^{\prime}}^{*} \\
& \alpha \Psi_{\mathrm{epw}}^{*}+\mathrm{i} \beta \frac{\partial \Psi_{\mathrm{epw}}^{*}}{\partial \mathrm{x}}=\gamma_{1} \Psi_{s^{\prime}} \\
& \alpha \Psi_{\mathrm{s}}^{*}-\mathrm{i} \eta \frac{\partial \Psi_{\mathrm{s}}^{*}}{\partial \mathrm{x}}=\gamma_{2} \Psi_{\mathrm{epw}}, \text { and } \\
& \alpha \Psi_{\mathrm{s}}+\mathrm{i} \eta \frac{\partial \Psi_{\mathrm{s}}}{\partial \mathrm{x}}=\gamma_{2} \Psi_{\mathrm{epw}}^{*}
\end{aligned}
$$

where $\quad \alpha=-\frac{\omega_{\mathrm{pe}}^{2}}{8}\left(\frac{1}{k_{\mathrm{epw}}^{2} \lambda_{\mathrm{pe}}^{2}}\left|\Psi_{\mathrm{epw}}\right|^{2}+\frac{\mathrm{c}^{2}}{\mathrm{v}_{\mathrm{th}}^{2}}\left|\Psi_{\mathrm{s}}\right|^{2}\right), \quad \beta=3 \mathrm{v}_{\mathrm{th}}^{2} \mathrm{k}_{\mathrm{epw}}, \quad \gamma_{1}=\frac{-\mathrm{c}^{2} \mathrm{k}_{\mathrm{epw}}^{2}}{4} \Psi_{0}$ ， $\eta=c^{2} k_{s}$, and $\gamma_{2}=\frac{-\omega_{\mathrm{pe}}^{2}}{4} \Psi_{\circ}$.

$$
\begin{aligned}
& \frac{\partial\left|\Psi_{\mathrm{epw}}\right|^{2}}{\partial \mathrm{x}}=\frac{-\eta}{\beta} \frac{\gamma_{1}}{\gamma_{2}} \frac{\partial\left|\Psi_{s}\right|^{2}}{\partial \mathrm{x}}, \\
& \mathrm{i} \frac{\partial}{\partial \mathrm{x}}\left(\Psi_{\mathrm{s}}^{*} \Psi_{\mathrm{epw}}^{*}+\Psi_{\mathrm{s}} \Psi_{\mathrm{epw}}\right)=\alpha\left(\frac{1}{\eta}-\frac{1}{\beta}\right)\left(\Psi_{\mathrm{s}}^{*} \Psi_{\mathrm{epw}}^{*}-\Psi_{s} \Psi_{\mathrm{epw}}\right), \\
& \mathrm{i} \frac{\partial}{\partial \mathrm{x}}\left(\Psi_{\mathrm{s}}^{*} \Psi_{\mathrm{epw}}^{*}-\Psi_{\mathrm{s}} \Psi_{\mathrm{epw}}\right)-\alpha\left(\frac{1}{\eta}-\frac{1}{\beta}\right)\left(\Psi_{\mathrm{s}}^{*} \Psi_{\mathrm{epw}}^{*}+\Psi_{\mathrm{s}} \Psi_{\mathrm{epw}}\right)= \\
& 2 \frac{\gamma_{1}}{\beta}\left|\Psi_{s}\right|^{2}-2 \frac{\gamma_{2}}{\eta}\left|\Psi_{\text {epw }}\right|^{2} \text {, and } \\
& \mathrm{i} \beta \frac{\partial\left|\Psi_{\mathrm{epw}}\right|^{2}}{\partial \mathrm{x}}=-\gamma_{1}\left(\Psi_{\mathrm{s}}^{*} \Psi_{\mathrm{epw}}^{*}-\Psi_{\mathrm{s}} \Psi_{\mathrm{epw}}\right) \text {. }
\end{aligned}
$$

These equations can be combined into a single equation

$$
\frac{\partial^{2}\left|\Psi_{\text {epw }}\right|^{2}}{\partial \mathrm{x}^{2}}+\frac{\alpha^{2}}{\beta^{2}}\left|\Psi_{\mathrm{epw}}\right|^{2}-\frac{4 \gamma_{1} \gamma_{2}}{\beta \eta}\left|\Psi_{\mathrm{epw}}\right|^{2}=0
$$


APPENDIX 2.5 SATURATION OF FORWARD RAMA . . . . . . . . 211

For $\left|\Psi_{\text {epw }}\right|^{2}<<1$, the solution to the above equation is approximately $\left|\Psi_{\text {ep }}\right|^{2} \propto \exp \left(2 \times \gamma_{o} / \sqrt{V_{s} V_{\text {ep }}}\right)$ where $\gamma_{o} / \sqrt{V_{s} V_{\text {ep }}}=\sqrt{\gamma_{1} \gamma_{2} /(\beta \eta)}$. Saturation happens when the left most terms in equation 2.5 .11 balance each other or when

$$
\left|\mathrm{n}_{\mathrm{epw}} / \mathrm{n}_{\mathrm{oe}}\right|=0.5 \mathrm{k} \lambda_{\mathrm{pe}} \sqrt{\frac{\gamma_{\mathrm{oSRS}}}{\omega_{\mathrm{pe}}}}\left(\frac{\mathrm{V}_{\mathrm{epw}}}{\mathrm{V}_{\mathrm{s}}}\right)^{0.25}
$$




\section{Appendix 2.6}

\section{Saturation of forward Raman scattering due to relativistic detuning}

This appendix details the derivation of the Langmuir wave's saturated amplitude due to relativistic detuning. The derivation presented in this appendix assumes that the laser intensity is small enough that the electrons do not experience relativistic motion due to the laser itself.

The equations describing stimulated Raman scattering can be derived from Maxwell's equations in conjunction with the relativistically correct fluid equations. The starting point for this derivation will be the relativistic momentum equation given by

$$
\frac{\partial \vec{\rho}}{\partial \mathrm{t}}+\overrightarrow{\mathrm{u}} \cdot \nabla \vec{\rho}=\mathrm{q}\left(\overrightarrow{\mathrm{E}}+\frac{\overrightarrow{\mathrm{u}}}{\mathrm{c}} \times \overrightarrow{\mathrm{B}}\right)-\frac{\nabla \mathrm{p}}{\mathrm{n}}
$$

where $\bar{\rho}=\frac{m_{0} \vec{u}}{\sqrt{1-u^{2} / c^{2}}}$. The resultant derivation will consider Raman forward scatter as a three wave process. Forward Raman scattering can involve four or more waves when this process occurs at low densities where the plasma 
frequency is much less than the incident laser frequency. The transverse components of the momentum equation above show that the transverse momentum, $\rho_{\perp}$, may expressed in terms of the vector potential, $A_{\perp}$, as $\rho_{\perp}=-q A_{\perp} / c$.

The equation representing the daughter Langmuir wave is

$$
\begin{aligned}
& \frac{\ddot{\zeta}\left(1-\left(\mathrm{u}_{\mathrm{os}} / \mathrm{c}\right)^{2}\right)}{\left(1-(\dot{\zeta} / c)^{2}-\left(\mathrm{u}_{\mathrm{os}} / \mathrm{c}\right)^{2}\right)^{3 / 2}}+\frac{\dot{\zeta}\left(\mathrm{u}_{\mathrm{os}} / \mathrm{c}\right)^{2} \partial\left(\mathrm{u}_{\mathrm{os}} / \mathrm{c}\right)^{2} / \partial t}{2\left(1-(\dot{\zeta} / c)^{2}-\left(\mathrm{u}_{\mathrm{os}} / \mathrm{c}\right)^{2}\right)^{3 / 2}}+ \\
& \left(\omega_{\mathrm{pe}}^{2}-3 \mathrm{v}_{\mathrm{th}}^{2} \nabla^{2}\right) \zeta=\frac{-\mathrm{q}^{2}}{\mathrm{~m}_{\mathrm{o}}^{2} \mathrm{c}^{2}} \sqrt{1-(\dot{\zeta} / c)^{2}-\left(\mathrm{u}_{\mathrm{os}} / \mathrm{c}\right)^{2}} \nabla\left(\dot{\mathrm{A}}_{\perp} \cdot \overrightarrow{\mathrm{A}}_{\mathrm{os}}\right),
\end{aligned}
$$

where $\zeta$ is the spatial displacement of the electron fluid in the wave and is related to the Langmuir wave amplitude by $\left|n_{\mathrm{re}} / \mathrm{n}_{\mathrm{oe}}\right|=|\nabla \zeta|$. The equation describing the electromagnetic daughter wave may be expressed as

$$
\begin{aligned}
& \left(\frac{\partial^{2}}{\partial \mathrm{t}^{2}}+\omega_{\mathrm{pe}}^{2} \sqrt{1-(\dot{\zeta} / c)^{2}-\left(\mathrm{u}_{\mathrm{os}} / \mathrm{c}\right)^{2}}-c^{2} \nabla^{2}\right) \mathrm{A}_{\perp}= \\
& -\omega_{\mathrm{pe}}^{2} \sqrt{1-(\dot{\zeta} / c)^{2}-\left(\mathrm{u}_{\mathrm{os}} / \mathrm{c}\right)^{2} \frac{\mathrm{n}_{\mathrm{e}}}{\mathrm{n}_{\mathrm{oe}}}} A_{o s}
\end{aligned}
$$

where $u_{O S}$ is the oscillation velocity of the electrons in the electromagnetic wave. Specifically, $u_{O}$ may be written in terms of the vector potential of the incident electromagnetic wave,

$$
\left(\mathrm{u}_{\mathrm{os}} / \mathrm{c}\right)^{2}=\left(1-(\dot{\zeta} / \mathrm{c})^{2}\right)\left(\mathrm{qA}_{\mathrm{os}} / \mathrm{m}_{\mathrm{e}} \mathrm{c}^{2}\right)^{2} /\left[1+\left(\mathrm{qA}_{\mathrm{os}} / \mathrm{m}_{\mathrm{e}} \mathrm{c}^{2}\right)^{2}\right] .
$$


After making the substitutions $\eta=\omega_{\mathrm{pe}} \zeta / \mathrm{c}, \tau=\omega_{\mathrm{pet}}, \chi=\omega_{\mathrm{pex}} / \mathrm{c}$, the vector potentials and Langmuir wave amplitude is written as a slowly varying component multiplied by a fast oscillating term,

$$
\begin{aligned}
& \Psi_{o s}=\left(q A_{o s} / m_{e} c^{2}\right)=0.5 * \Psi_{o}(t)\left[\exp \left(\mathrm{ik}_{o} c \chi / \omega_{p e}-i \omega_{o} t / \omega_{p e}\right)+c . c .\right] \\
& \Psi_{\perp}=\left(q A_{\perp} / m_{e} c^{2}\right)=0.5\left[\Psi_{s}(t)^{*} \exp \left(\mathrm{ik}_{\mathrm{s}} c \chi / \omega_{p e}-i \omega_{s} t / \omega_{p e}\right)+c . c .\right] \\
& \text { and } \eta=0.5\left[\xi(t)^{*} \exp \left(\mathrm{ik}_{\mathrm{p}} c \chi / \omega_{p e}-i \omega_{p} t / \omega_{p e}\right)+c . c .\right]
\end{aligned}
$$

Using the dispersion relations of the waves, equations $2.6 .2,2.6 .3$, and 2.6 .5 can be reduced to

$$
\begin{aligned}
& -\left(\frac{\omega_{p}}{\omega_{p e}}\right)^{2} \frac{|\xi|^{2}}{8} \Psi_{s}-i \frac{\omega_{s}}{\omega_{p e}} \dot{\Psi}_{s}=\frac{-i k_{p} c}{4 \omega_{p e}} \xi^{*} \Psi_{o}\left(1-\left(\frac{\omega_{p}}{\omega_{p e}}\right)^{2} \frac{|\xi|^{2}}{4}\right), \\
& -\left(\frac{\omega_{p}}{\omega_{p e}}\right)^{2} \frac{|\xi|^{2}}{8} \Psi_{s}^{*}+i \frac{\omega_{s}}{\omega_{p e}} \dot{\Psi}_{s}^{*}=\frac{i k_{p} c}{4 \omega_{p e}} \xi \Psi_{o}\left(1-\left(\frac{\omega_{p}}{\omega_{p e}}\right)^{2} \frac{|\xi|^{2}}{4}\right), \\
& -\frac{3}{8}\left(\frac{\omega_{p}}{\omega_{p e}}\right)^{4}|\xi|^{2} \xi-i \frac{\omega_{p}}{\omega_{p e}} \dot{\xi}=\frac{-i\left(k_{o}-k_{s}\right) c}{\omega_{p e}} \frac{\Psi_{o} \Psi_{s}^{*}}{4}\left(1-\left(\frac{\omega_{p}}{\omega_{p e}}\right)^{2} \frac{|\xi|^{2}}{4}\right) \text {, and } \\
& -\frac{3}{8}\left(\frac{\omega_{p}}{\omega_{p e}}\right)^{4}|\xi|^{2} \xi^{*}+i \frac{\omega_{p}}{\omega_{p e}} \dot{\xi}^{*}=\frac{i\left(k_{o}-k_{s}\right) c}{\omega_{p e}} \frac{\Psi_{0} \Psi_{s}}{4}\left(1-\left(\frac{\omega_{p}}{\omega_{p e}}\right)^{2} \frac{|\xi|^{2}}{4}\right) .
\end{aligned}
$$

These four equations may be simplified combined and written as 


$$
\begin{aligned}
& \frac{d A}{d \tau}=\alpha C \\
& \frac{d A}{d \tau}=\frac{\omega_{s}}{\omega_{p}} \frac{d B}{d \tau} \\
& \frac{d C}{d \tau}-i \pi A D=2 \alpha \frac{\omega_{p}}{\omega_{s}}\left[\frac{\omega_{s}}{\omega_{p}} B+A\right], \text { and } \\
& \frac{d D}{d \tau}-i \pi A C=0 .
\end{aligned}
$$

In equation 2.6.7 above, $\mathrm{A}=|\xi|^{2}, \mathrm{~B}=\left|\Psi_{\mathrm{s}}\right|^{2}, \mathrm{C}=\left[\xi \Psi_{\mathrm{s}}+\xi^{*} \Psi_{\mathrm{s}}^{*}\right], \mathrm{D}=\left[\xi \Psi_{\mathrm{s}}-\xi^{*} \Psi_{\mathrm{s}}^{*}\right]$, $\alpha(\mathrm{A})=\left(1-\left(\frac{\omega_{\mathrm{p}}}{\omega_{\mathrm{pe}}}\right)^{2} \frac{|\xi|^{2}}{4}\right) \frac{\mathrm{k}_{\mathrm{p}} \mathrm{c} \Psi_{\mathrm{o}}}{4 \omega_{\mathrm{p}}}$, and $\pi=\left[\frac{3}{8}\left(\frac{\omega_{\mathrm{p}}}{\omega_{\mathrm{pe}}}\right)^{3}+\frac{1}{8}\left(\frac{\omega_{\mathrm{p}}}{\omega_{\mathrm{pe}}} \frac{\omega_{\mathrm{p}}}{\omega_{\mathrm{s}}}\right)\right]$. The second equation in 2.6.7 expresses conservation of action. The four equation in 2.6.7 can be used to eliminate all of the variables except $A$, resulting in the equation

$$
\frac{\mathrm{d}^{2} \mathrm{~A}}{\mathrm{~d} \tau^{2}}-4 \alpha^{2} \frac{\omega_{\mathrm{p}}}{\omega_{\mathrm{s}}} \mathrm{A}+\frac{\pi^{2}}{2} \mathrm{~A}^{3}=0
$$

For $A<<1$, the Langmuir wave amplitude grows like $A \approx A_{o} e^{2 n}$, where the homogeneous growth rate $\gamma=\alpha \omega_{\mathrm{pe}} \sqrt{\frac{\omega_{\mathrm{p}}}{\omega_{\mathrm{s}}}}$. Saturation happens when the left most terms in equation 2.6 .8 are equal or when $A=\frac{\sqrt{8}}{\pi} \frac{\gamma}{\omega_{\mathrm{pe}}}$. The saturation amplitude of the Langmuir wave is therefore,

$$
\frac{n_{1 e}}{n_{o e}}=\frac{k_{p} c 8^{0.25}}{\sqrt{2} \omega_{p e}}\left[\frac{3}{8}\left(\frac{\omega_{p}}{\omega_{p e}}\right)^{3}+\frac{1}{8}\left(\frac{\omega_{p}}{\omega_{p e}} \frac{\omega_{p}}{\omega_{s}}\right)\right]^{-1} \sqrt{\frac{\gamma}{\omega_{p e}}} \approx 2 \sqrt{\frac{\gamma}{\omega_{p e}}}
$$




\section{Appendix 3.1}

\section{Numerical calculation of the scattering version of EDI from an inhomogeneous plasma}

This appendix details the numerical calculation for the scattering version of the electromagnetic decay instability. From chapter 3 , the equation describing the growth of the scattered vector potential is

$$
\left(\frac{\partial^{2}}{\partial t^{2}}+\omega_{\mathrm{pe}}^{2}\left(1+\frac{x}{L_{n}}\right)-c^{2} \frac{\partial^{2}}{\partial x^{2}}\right) \frac{q A_{s c}}{m_{e} c^{2}}=-\omega_{p e}^{2} \frac{n_{i a}}{n_{o e}}\left(\frac{\omega_{\text {epw }}}{c k_{\text {epw }}} \frac{n_{e p w}}{n_{o e}}\right)
$$

where $n_{e p w} / n_{o e}$ and $n_{i a} / n_{o e}$ are the pump Langmuir wave amplitude and the ion acoustic amplitude, respectively and $A_{S C}$ is the vector potential of the scattered light wave. The pump Langmuir wave and ion acoustic wave amplitudes are assumed to be of the form $\frac{\mathrm{n}_{\alpha}}{\mathrm{n}_{\mathrm{oe}}}=\frac{1}{2} \Psi_{\alpha}(\mathrm{x}) \exp \left[\mathrm{i}\left(\int \overrightarrow{\mathrm{k}}_{\alpha} \cdot \mathrm{d} \overrightarrow{\mathrm{x}}-\omega_{\alpha} \mathrm{t}\right)\right]+$ c.c. The scattered vector potential is assumed to be of the form $\frac{\mathrm{qA}_{\mathrm{sc}}}{\mathrm{m}_{\mathrm{e}} \mathrm{c}^{2}}=\frac{1}{2} \Psi_{s}(\mathrm{x}) \exp \left[-\mathrm{i} \omega_{\mathrm{s}} t\right]+\mathrm{c}$.c.. The steady state form of equation 3.1.1 can be written as 


$$
\left(\frac{\mathrm{d}^{2}}{d x^{2}}-\frac{\omega_{\mathrm{pe}}^{2} \mathrm{x}}{\mathrm{c}^{2} \mathrm{~L}_{\mathrm{n}}}\right) \Psi_{\mathrm{s}}(\mathrm{x})=\frac{\omega_{\mathrm{pe}}^{2} \omega_{\mathrm{epw}}}{2 \mathrm{c}^{3} \mathrm{k}_{\mathrm{epw}}}\left(\Psi_{\mathrm{epw}} \Psi_{\mathrm{ia}}\right) \exp \left[\frac{\mathrm{i} \kappa^{\prime} \mathrm{x}^{2}}{2}\right]
$$

where the wavenumber mismatch of the pump Langmuir wave and the ion acoustic wave has been Taylor expanded, $\left(\vec{k}_{i a}(x)+\vec{k}_{\text {epw }}(x)\right) \cdot d \vec{x} \approx K^{\prime} x$, about the perfect phase matching point. The spatial coordinate is normalized according to $\chi=k^{\prime 0.5} x$. Equation 3.1 .2 can then be written as

$$
\left(\frac{\mathrm{d}^{2}}{\mathrm{~d} \chi^{2}}-\xi \chi\right) \Psi_{s}(\chi)=\sigma \exp \left[\frac{\mathrm{i} \chi^{2}}{2}\right]
$$

where $\sigma=\omega_{\mathrm{pe}}^{2} \omega_{\mathrm{epw}}\left(\Psi_{\mathrm{epw}} \Psi_{\mathrm{ia}}\right)\left(2 c^{3} \mathrm{k}_{\mathrm{epw}} \kappa^{\prime}\right)^{-1} \quad$ and $\quad \xi=\omega_{\mathrm{pe}}^{2}\left(c^{2} \mathrm{~L}_{\mathrm{n}} \kappa^{\prime 1.5}\right)^{-1}$. Equation 3.1.3 was treated as a boundary value problem in which $\Psi_{S}=0$ at the right hand boundary and is an outgoing wave at the left hand boundary. Equation 3.1 .3 can be differenced into the equation

$$
\begin{aligned}
& \left(1-0.25(\Delta \chi)^{2} \xi \chi_{\mathrm{n}}\right) \Psi_{\mathrm{n}+1}+\left(-2-0.5(\Delta \chi)^{2} \xi \chi_{\mathrm{n}}\right) \Psi_{\mathrm{n}}+ \\
& \left(1-0.25(\Delta \chi)^{2} \xi \chi_{\mathrm{n}}\right) \Psi_{\mathrm{n}-1}=\sigma \exp \left[\frac{\mathrm{i} \chi_{\mathrm{n}}^{2}}{2}\right]
\end{aligned}
$$

Equation 3.1.4 represents a tri-diagonal matrix which can be solved assuming a one term backward recursion relation of the form $\Psi_{n-1}=\alpha_{n} \Psi_{n}+\beta_{n}$. Substitution of the recurrence relation into equation 3.1.4 allows for the determination of the recursion relations for the $\alpha$ and $\beta$ coefficients. This procedure yields 


$$
\begin{aligned}
& \alpha_{\mathrm{n}}=\frac{-\left(1-0.25(\Delta \chi)^{2} \xi \chi_{\mathrm{n}-1}\right)}{\left(1-0.25(\Delta \chi)^{2} \xi \chi_{\mathrm{n}-1}\right) \alpha_{\mathrm{n}-1}+\left(-2-0.5(\Delta \chi)^{2} \xi \chi_{\mathrm{n}-1}\right)} \text { and } \\
& \beta_{\mathrm{n}}=\frac{\sigma \exp \left[\mathrm{i} 0.5 \chi_{\mathrm{n}-1}^{2}\right]-\left(1-0.25(\Delta \chi)^{2} \xi \chi_{\mathrm{n}-1}\right) \beta_{\mathrm{n}-1}}{\left(1-0.25(\Delta \chi)^{2} \xi \chi_{\mathrm{n}-1}\right) \alpha_{\mathrm{n}-1}+\left(-2-0.5(\Delta \chi)^{2} \xi \chi_{\mathrm{n}-1}\right)} .
\end{aligned}
$$

The outgoing boundary condition on the left hand side fixes the value of $\alpha_{\text {-nstep }+1}=\left(1+\mathrm{i} 0.5(\Delta \chi) \sqrt{\xi \chi_{\text {nstep-1 }}}\right) /\left(1-\mathrm{i} 0.5(\Delta \chi) \sqrt{\xi \chi_{\text {nstep-1 }}}\right)$ and $\beta_{\text {-nstep+1 }}=0$ with the inclusion of a gaussian damping factor on the right hand side of equation 3.1.3 as shown in the program.

The actual program follows:

C This program will look at the amplitude of the C scattered signal due to a linear phase mismatch

$C$ when the scattered signal is near its turning point.

PARAMETER (NSTEP $=6000$ )

COMPLEX ScaOld(-NSTEP:NSTEP),ScaNew(0:51),

!i,Disp(-NSTEP:NSTEP),

!Alpha(-NSTEP:NSTEP),Beta(-NSTEP:NSTEP)

REAL sigma,eta,DX,cie

Integer $\mathrm{k}$

open(unit=13,file='steady', status='unknown')

close(unit=13,status='delete')

open(unit=13,file='steady', status='unknown')

open(unit $=14$,file='sigmavary',status='unknown')

close(unit=14,status='delete')

open(unit=14,file='sigmavary',status='unknown')

$\mathrm{i}=\mathrm{CMPLX}(0 ., 1$.)

14 PRINT *, 'ENTER sigma,eta,cie'

16 PRINT *, 'SUGGEST 0.005,0.1,0.0'

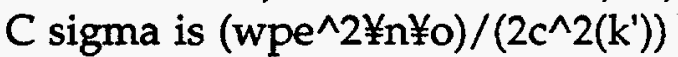

$C$ eta is wpe^ $2 /\left(c^{\wedge} 2 \operatorname{Ln}\left(k^{\prime}\right)^{\wedge} 1.5\right)$

$C$ cie is normalized $k s^{\wedge} 2=\left(w s^{\wedge} 2-w p e^{\wedge} 2\right) /\left(c^{\wedge} 2\left(k^{\prime}\right)\right)$ 
C normalized time $t^{\prime}=t\left(w p e^{\wedge} 2 ¥ n ¥ o\right) /(4 w s)$

$C$ normalized space $x^{\prime}=x\left(k^{\prime}\right)^{\wedge} 0.5$

READ *, sigma,eta,cie

$\mathrm{DX}=0.01$

$C$ outgoing boundary conditions on right-hand side

$\mathrm{C}$ alpha(i) $=-(\mathrm{A}+) /\left(\right.$ alpha $\left.(\mathrm{i}-1)^{*}(\mathrm{~A}-)+(\mathrm{A} 0)\right)$

$\mathrm{CA}-=$ sigma $/ \mathrm{DX}{ }^{\wedge} 2$

$\mathrm{C} \mathrm{A} 0=-2$ sigma $/ \mathrm{DX} \wedge 2+\mathrm{i} / \mathrm{DT}$

$\mathrm{CA}+=$ sigma $/ \mathrm{DX} \wedge 2$

Alpha $(-N S T E P+1)=(1.0+$

!i*0.5*DX*(eta*DX*Real(NSTEP-1) $\left.)^{* *} 0.5\right) /$

! $\left(1.0-\mathrm{i}^{*} 0.5^{*} \mathrm{DX} \mathrm{X}^{*}\left(\mathrm{eta} \mathrm{DX}^{*}\right.\right.$

(Real(NSTEP-1)) ${ }^{* *} 0.5$ )

C Alpha(-NSTEP+1) $=$ CMPLX $(0.000,0$.)

$C$ end boundary conditions

$C$ setup initial wave amplitudes

DO 20 IX $=-$ NSTEP,NSTEP

ScaOld(IX) $=$ CMPLX $(0.000,0$.

$\operatorname{Disp}(\mathrm{IX})=\mathrm{i}^{*} \mathrm{DX}^{* *} 2.0^{*}(\mathrm{REAL}(\mathrm{IX}))^{* *} 2.0 / 2.0$

! $-\left(4.8^{*}(\right.$ REAL(IX)) $/$ REAL(NSTEP) $) * * 2.0$

20 CONTINUE

$C$ end setup initial wave amplitudes

DO $30 \mathrm{~J}=-\mathrm{NSTEP}+2$,NSTEP, 1

Alpha $(J)=\left(-1.0+i^{*} 0.5^{*} \mathrm{cie}^{*} \mathrm{DX}+\right.$

! $\mathrm{DX}^{* * 2.0^{*} \text { eta* }}$

! REAL(J-1)*DX/4.0)/

! (Alpha(J-1)*(1.0+i*0.5* cie*DX-

! DX*2.0*eta*

! REAL(J-1)*DX/4.0)-2.0-

! $\mathrm{DX}^{* *} 2.0^{*}$ eta*

! REAL(J-1)*DX/2.0)

30 CONTINUE

$\mathrm{C}$ beta $(\mathrm{i})=(\mathrm{b}(\mathrm{i}-1)-(\mathrm{A}-) \mathrm{beta}(\mathrm{i}-1))$

$\mathrm{C} /($ alpha(i-1)*(A-)+(A0))

$C b(\mathrm{i}-1)=(\mathrm{i} / \mathrm{DT}$-cie+eta*DX*(i-1))ScaOld(i-1)+

$C \operatorname{cexp}\left(0.5 i x^{\wedge} 2+\mathrm{ix}(\mathrm{cie} / \mathrm{sigma})^{\wedge} 0.5\right)$

Beta(-NSTEP+1) $=$ CMPLX $(0.000,0$.)

DO 35 sigma $=0.001,0.05,0.001$

DO $45 \mathrm{~J}=-\mathrm{NSTEP}+2, \mathrm{NSTEP}, 1$

$\operatorname{Beta}(\mathrm{J})=\left(\mathrm{DX} * 2.0^{*}\right.$ sigma*CEXP(Disp $\left.(\mathrm{J}-1)\right)-$

! Beta $(\mathrm{J}-1)^{*}\left(1.0+\mathrm{i}^{*} 0.5^{*} \mathrm{Cie}^{*} \mathrm{DX}-\right.$

! $\mathrm{DX}^{* *} 2.0^{*} \mathrm{eta}^{*}$

! REAL(J-1)*DX/4.0))/ 
! (Alpha(J-1)* $\left(1.0+\mathrm{i}^{*} 0.5^{*} \mathrm{cie}^{*} \mathrm{DX}-\right.$

! DX*2.0*eta*

! REAL(J-1)*DX/4.0)-2:0-

! DX*2.0*eta*

! REAL(J-1)*DX/2.0)

45 CONTINUE

$C$ fill out amplitude arrays at new time step

C $¥(\mathrm{i}-1)=$ alpha $(\mathrm{i}) ¥(\mathrm{i})+$ beta(i)

DO 50 IX=NSTEP, -NSTEP+1, -1

ScaOld(IX-1) $=$ Alpha(IX)*ScaOld(IX)+

! Beta(IX)

50 CONTINUE

$\mathrm{k}=1000^{*}$ sigma

$\mathrm{ScaNew}(\mathrm{k})=\mathrm{ScaOld}(0)$

35 Continue

write $\left(13,{ }^{*}\right)$,'Mag. of As',',',',position'

DO $70 \mathrm{JX}=-\mathrm{NSTEP}$, NSTEP, 20

write $(13, *), \mathrm{CABS}(\mathrm{ScaOld}(\mathrm{JX})),,^{\prime}$,

! JX*DX

70 Continue write $\left(14,{ }^{*}\right)$,'Mag. of As',',','sigma'

DO 80 sigma $=0.001,0.05,0.001$

$\mathrm{k}=1000^{*}$ sigma

write $(14, *), \mathrm{CABS}(\mathrm{ScaNew}(\mathrm{k})),,$, ,

! sigma

80 Continue

close(unit=13)

close(unit=14)

END 



\section{Appendix 4.1}

\section{Derivation of the Thomson scattered signal using the fluid equations}

This appendix details the derivation of the equation describing the vector potential of the Thomson scattered signal using Maxwell's equations in conjunction with the electron fluid equations. This is a simpler approach showing the important parameters for Thomson scattering in plasmas. To correctly recover the Thomson scattering features, a kinetic approach must be used to recover the correct spectral features of the density fluctuations. This kinetic approach is reviewed in Appendix 4.2.

The first step is to derive the equation describing the Thomson scattered vector potential[16]. The derivation begins with Ampere's law,

$$
\nabla \times \vec{B}=\frac{4 \pi}{c} \vec{J}+\frac{1}{c} \frac{\partial \vec{E}}{\partial t}
$$


which relates the magnetic field, $\vec{B}$, to the current, $\vec{J}$, and the rate of change of the electric field, $\overrightarrow{\mathrm{E}}$. Inserting the relations $\overrightarrow{\mathrm{B}}=\nabla \times \overrightarrow{\mathrm{A}}$, and $\overrightarrow{\mathrm{E}}=-\nabla \phi-\frac{1}{\mathrm{c}} \frac{\partial \overrightarrow{\mathrm{A}}}{\partial \mathrm{t}}$ into Ampere's law yields

$$
\frac{\partial^{2} \vec{A}}{\partial t^{2}}-c^{2} \nabla^{2} \vec{A}=4 \pi c \vec{\jmath}-c^{2} \nabla\left(\nabla \cdot \vec{A}+\frac{1}{c} \frac{\partial \phi}{\partial t}\right)
$$

relating the vector potential, $\overrightarrow{\mathrm{A}}$, to the current and the scalar potential, $\phi$. From equation 4.1.2, the transverse component of the scattered vector potential, $\vec{A}_{t}$, can be written as

$$
\frac{\partial^{2} \overrightarrow{\mathrm{A}}_{\mathrm{t}}}{\partial \mathrm{t}^{2}}-c^{2} \nabla^{2} \overrightarrow{\mathrm{A}}_{\mathrm{t}}=4 \pi c \overrightarrow{\mathrm{J}}_{\mathrm{t}}
$$

The transverse current, $\overrightarrow{\mathrm{J}}_{\mathrm{t}}$, is found from the electron momentum equation

$$
\frac{\partial \overrightarrow{\mathrm{u}}}{\partial \mathrm{t}}-\frac{1}{2} \nabla(\overrightarrow{\mathrm{u}} \cdot \overrightarrow{\mathrm{u}})-\overrightarrow{\mathrm{u}} \times(\nabla \times \overrightarrow{\mathrm{u}})=\frac{-\mathrm{e}}{\mathrm{m}_{\mathrm{e}}}\left[-\nabla \phi-\frac{1}{\mathrm{c}} \frac{\partial \overrightarrow{\mathrm{A}}}{\partial \mathrm{t}}+\frac{\overrightarrow{\mathrm{u}}}{\mathrm{c}} \times(\nabla \times \overrightarrow{\mathrm{A}})\right]-\frac{\nabla \mathrm{p}_{\mathrm{e}}}{\mathrm{n}_{\mathrm{e}} \mathrm{m}_{\mathrm{e}}},
$$

where $\vec{u}, p_{e}$, and $n_{e}$ are the electron fluid velocity, pressure, and density respectively. Upon inspection of equation 4.1 .4 , it is apparent that the transverse electron fluid velocity, $\vec{u}_{t}$, is equal to $\vec{u}_{t}=e \vec{A} / \mathrm{cm}_{e}$, provided the damping is small, where $e$ and $m_{e}$ are the magnitude of the electron's charge and mass respectively. Combining the above formula for the transverse current with equation 4.1 .3 , the scattered vector potential can then be written as 


$$
\frac{\partial^{2} \overrightarrow{\mathrm{A}}_{t}}{\partial \mathrm{t}^{2}}-c^{2} \nabla^{2} \overrightarrow{\mathrm{A}}_{\mathrm{t}}=-\frac{4 \pi \mathrm{e}^{2}}{\mathrm{~m}_{\mathrm{e}}} \mathrm{n}_{\mathrm{e}} \overrightarrow{\mathrm{A}}_{\mathrm{t}}
$$

The electron fluid density, $n_{e}$, is separated into a zero-order background density, $n_{o e}$, and a first-order perturbation, $n_{e p w}$, representing the Langmuir wave fluctuation present in the plasma. Next the transverse vector potential, $\vec{A}_{t}$, is separated into a zero-order component representing the incident Thomson probe beam, Aprobe, and a first-order component representing the Thomson scattered vector potential, Ascatt. The resulting equation describing the growth of the Thomson scattered vector potential is

$$
\begin{aligned}
& \left(\frac{\partial^{2}}{\partial t^{2}}+v \frac{\partial}{\partial t}+\omega_{p e}^{2}-c^{2} \nabla^{2}\right) \frac{q A_{s c a t t}}{m_{e} c^{2}}= \\
& -\frac{4 \pi n_{o e} e^{2}}{m_{e}}\left(\hat{e}_{\text {scatt }} \cdot \hat{e}_{\text {probe }}\right) \frac{n_{\text {epw }}}{n_{o e}} \frac{\mathrm{qA}_{\text {probe }}}{m_{e} c^{2}},
\end{aligned}
$$

where $\hat{\mathrm{e}}_{\text {scatt }}$ and $\hat{\mathrm{e}}_{\text {probe }}$ are unit vectors in the direction of the scattered and probe vector potential respectively.

Equation 4.1 .6 can be simplified by assuming that the vector potentials and density fluctuation can be written in the form $\mathrm{qA}_{\text {scatt }} / \mathrm{m}_{\mathrm{e}} \mathrm{c}^{2}=0.5 \Psi_{\mathrm{s}}(\mathrm{x}) \exp \left(\mathrm{i}_{\mathrm{s}} \cdot \overrightarrow{\mathrm{x}}-\mathrm{i} \omega_{\mathrm{s}} \mathrm{t}\right)+\mathrm{c.c}$. $\mathrm{n}_{\mathrm{epw}} / \mathrm{n}_{\mathrm{oe}}=0.5 \Psi_{\mathrm{e}}(\mathrm{x}) \exp \left(\mathrm{i} \overrightarrow{\mathrm{k}}_{\mathrm{e}} \cdot \overrightarrow{\mathrm{x}}-\mathrm{i} \omega_{\mathrm{e}} \mathrm{t}\right)+$ c.c., a $\quad n \quad d$ $\mathrm{qA}_{\text {probe }} / \mathrm{m}_{\mathrm{e}} \mathrm{c}^{2}=0.5 \Psi_{\mathrm{pr}}(\mathrm{x})\left\{\exp \left(\mathrm{i} \overrightarrow{\mathrm{k}}_{\mathrm{pr}} \cdot \overrightarrow{\mathrm{x}}-\mathrm{i} \omega_{\mathrm{pr}} \mathrm{t}\right)+\mathrm{c} . \mathrm{c}\right\}$. The equation describing the scattered vector potential can be written as 


$$
\left[\beta+v_{\mathrm{gs}} \frac{\mathrm{d}}{\mathrm{dx}}\right] \Psi_{\mathrm{s}}(\mathrm{x})=\frac{-\omega_{\mathrm{pe}}^{2}\left(\hat{\mathrm{e}}_{\mathrm{sc}} \cdot \hat{\mathrm{e}}_{\mathrm{pr}}\right)}{-4 i \omega} \Psi_{\mathrm{pr}} \Psi_{\mathrm{e}}(\mathrm{x})
$$

where $\beta=v+\left(-\omega^{2}+\omega_{\mathrm{pe}}^{2}-c^{2} \mathrm{k}^{2}\right) /-4 \mathrm{i} \omega$. Assuming that the density fluctuation is independent of $x$, this scattered vector potential from a finite plasma of length $L$ is given by

$$
\Psi_{s}(\mathrm{~L})=\left[\frac{\omega_{\mathrm{pe}}^{2}\left(\hat{\mathrm{e}}_{\mathrm{sc}} \cdot \hat{\mathrm{e}}_{\mathrm{pr}}\right)}{4 \mathrm{i} \omega \beta} \Psi_{\mathrm{pr}} \Psi_{\mathrm{e}}\right]\left[1-\exp \left(-\mathrm{L} \beta / \mathrm{v}_{\mathrm{gs}}\right)\right]
$$

For a finite plasma with small damping on the scattered electromagnetic wave, such that $L \beta / v g s<<1$, the scattered vector potential can be written as

$$
\Psi_{\mathrm{s}}(\mathrm{L})=\frac{\omega_{\mathrm{pe}}^{2}\left(\hat{\mathrm{e}}_{\mathrm{sc}} \cdot \hat{\mathrm{e}}_{\mathrm{pr}}\right)}{4 \mathrm{i} \omega \mathrm{v}_{\mathrm{gs}}} \Psi_{\mathrm{pr}} \Psi_{\mathrm{e}} \mathrm{L}
$$

which is independent of the damping on the light wave. In the limit that the parameter $L \beta / v g s>>1$, the scattered vector potential can be written as

$$
\Psi_{\mathrm{s}}(\mathrm{L})=\frac{\omega_{\mathrm{pe}}^{2}\left(\hat{\mathrm{e}}_{\mathrm{sc}} \cdot \hat{\mathrm{e}}_{\mathrm{pr}}\right)}{4 \mathrm{i} \omega \beta} \Psi_{\mathrm{pr}} \Psi_{\mathrm{e}}
$$

The intensity of Thomson scattered light from a plasma is given by

$$
I(\vec{x}, t)=v_{g} \frac{\left\langle E(\vec{x}, t) E^{*}(\vec{x}, t)\right\rangle}{8 \pi}=k_{s} \omega_{s}\left(\frac{m_{e} c^{2}}{q}\right)^{2} \frac{\left\langle A_{s}(\vec{x}, t) A_{s}^{*}(\vec{x}, t)\right\rangle}{8 \pi} .
$$


The expectation value of the vector potential product can be written in terms of the expectation of the Fourier transformed vector potentials, or

$$
\begin{array}{r}
\left\langle A_{s}(\vec{x}, t) A_{s}^{*}(\vec{x}, t)\right\rangle=\left(\frac{1}{2 \pi}\right)^{8} \iiint \int\left\langle A_{s}(\omega, \vec{k}) A_{s}^{*}\left(\omega^{\prime}, \vec{k}^{\prime}\right)\right\rangle \\
\left.e^{i\left[\left(\vec{k}-\vec{k}^{\prime}\right) \cdot \vec{x}-\left(\omega-\omega^{\prime}\right) t\right.}\right]_{d \vec{k} d \omega d \vec{k}^{\prime} d \omega^{\prime} .}
\end{array}
$$

As discussed in Oberman and Auer[90], the ensemble average of quantities may be written as

$$
\begin{aligned}
& \left\langle\alpha_{\sigma}\left(x_{i o}, v_{i}\right) \alpha_{\sigma^{\prime}}\left(x_{j o}, v_{j}\right)\right\rangle=\iiint \int\left(\frac{d^{3} x_{i}}{v}\right)\left(\frac{d^{3} x_{j}}{v}\right) d^{3} v_{i} d^{3} v_{j} \\
& \alpha_{\sigma}\left(x_{i o}, v_{i}\right) \alpha_{\sigma^{\prime}}\left(x_{j o}, v_{j}\right) f_{\sigma}\left(\vec{v}_{i}\right) f_{\sigma^{\prime}}\left(\vec{v}_{j}\right) .
\end{aligned}
$$

From equation 4.2.7 in Appendix 4.2, the Fourier transformed scattered vector potential is proportional to

$$
\begin{aligned}
\mathrm{A}_{s}(\omega, \overrightarrow{\mathrm{k}}) \propto & \mathrm{n}_{1 \mathrm{e}}(\omega, \overrightarrow{\mathrm{k}})=\left\{1-\frac{\chi_{\mathrm{e}}(\omega, \overrightarrow{\mathrm{k}})}{\varepsilon(\omega, \overrightarrow{\mathrm{k}})}\right\} \sum_{\text {electrons }} \frac{\mathrm{e}^{-\mathrm{i} \overrightarrow{\mathrm{k}} \cdot \overrightarrow{\mathrm{x}}_{\circ}} \delta\left(\omega-\overrightarrow{\mathrm{k}} \cdot \overrightarrow{\mathrm{v}}_{\mathrm{o}}\right)}{(2 \pi)^{3}}+ \\
& \left\{\frac{-\chi_{\mathrm{e}}(\omega, \overrightarrow{\mathrm{k}})}{\varepsilon(\omega, \overrightarrow{\mathrm{k}})}\right\} \sum_{\alpha} \sum_{\text {ions }} \frac{\mathrm{Z}_{\alpha} \mathrm{e}^{-\mathrm{i} \overrightarrow{\mathrm{k}} \cdot \overrightarrow{\mathrm{x}}_{\circ}} \delta\left(\omega-\overrightarrow{\mathrm{k}} \cdot \overrightarrow{\mathrm{v}}_{\mathrm{o}}\right)}{(2 \pi)^{3}}
\end{aligned}
$$

Because the electrons and ions, as well as different ion species, are assumed to be uncorrelated, each species can be treated separately. The ensemble average 
of the product of the Fourier transformed density fluctuations can be written as

$$
\begin{aligned}
& \left.\left\langle n_{1}(\omega, \vec{k}) n_{1}^{*}\left(\omega^{\prime}, \vec{k}^{\prime}\right)\right\rangle=\frac{1}{(2 \pi)^{3}} \iiint \int \frac{d^{3} x_{i}}{v}\right)\left(\frac{d^{3} x_{j}}{v}\right) d^{3} v_{i} d^{3} v_{j} f_{\sigma}\left(\vec{v}_{i}\right) \\
& \mathbf{f}_{\sigma^{\prime}}\left(\overrightarrow{\mathrm{v}}_{\mathbf{j}}\right)\left[\left\{1-\frac{\chi_{\mathrm{e}}(\omega, \overrightarrow{\mathrm{k}})}{\varepsilon(\omega, \overrightarrow{\mathrm{k}})}\right\}^{2} \sum_{\mathbf{i}} \mathrm{e}^{-\mathrm{i} \overrightarrow{\mathrm{k}} \cdot \overrightarrow{\mathrm{x}}_{i}} \delta\left(\omega-\overrightarrow{\mathrm{k}} \cdot \overrightarrow{\mathrm{v}}_{\mathbf{i}}\right) \sum_{\mathbf{j}} \mathrm{e}^{-\mathrm{i} \overrightarrow{\mathrm{k}}^{\prime} \cdot \overrightarrow{\mathrm{x}}_{\mathrm{i}}} \delta\left(\omega^{\prime}-\overrightarrow{\mathrm{k}}^{\prime} \cdot \overrightarrow{\mathrm{v}}_{\mathbf{j}}\right)+\right. \\
& \left.\left\{\frac{-\chi \mathrm{e}(\omega, \overrightarrow{\mathrm{k}})}{\varepsilon(\omega, \overrightarrow{\mathrm{k}})}\right\}^{2} \sum_{\alpha} \sum_{\mathrm{i}} \mathrm{z}_{\alpha} \mathrm{e}^{-\mathrm{i} \overline{\mathrm{k}} \cdot \overrightarrow{\mathrm{x}}_{\mathrm{i}}} \delta\left(\omega-\overrightarrow{\mathrm{k}} \cdot \overrightarrow{\mathrm{v}}_{\mathrm{i}}\right) \sum_{\mathrm{j}} \mathrm{z}_{\alpha} \mathrm{e}^{-\mathrm{i} \overrightarrow{\mathrm{k}}^{\prime} \cdot \overrightarrow{\mathrm{x}}_{\mathrm{i}}} \delta\left(\omega^{\prime}-\overrightarrow{\mathrm{k}}^{\prime} \cdot \overrightarrow{\mathrm{v}}_{\mathrm{j}}\right)\right]
\end{aligned}
$$

Equation 4.1 .15 can be reduced to

$$
\begin{aligned}
& \left\langle\mathrm{n}_{1}(\omega, \overrightarrow{\mathrm{k}}) \mathrm{n}_{1}^{*}\left(\omega^{\prime}, \overrightarrow{\mathrm{k}}^{\prime}\right)\right\rangle=\frac{\mathrm{n}_{\mathrm{oe}}}{(2 \pi)^{3} \mathrm{k}} \delta\left(\overrightarrow{\mathrm{k}}-\overrightarrow{\mathrm{k}}^{\prime}\right) \delta\left(\omega-\omega^{\prime}\right) \\
& {\left[\left\{1-\frac{\chi_{\mathrm{e}}(\omega, \overrightarrow{\mathrm{k}})}{\varepsilon(\omega, \overrightarrow{\mathrm{k}})}\right\}^{2} \mathrm{f}_{\mathrm{oe}}\left(\frac{\omega}{\mathrm{k}}\right)+\left\{\frac{-\chi_{\mathrm{e}}(\omega, \overrightarrow{\mathrm{k}})}{\varepsilon(\omega, \overrightarrow{\mathrm{k}})}\right\}^{2} \sum_{\alpha} \mathrm{z}_{\alpha}^{2} \mathrm{f}_{\mathrm{oi} \alpha}\left(\frac{\omega}{\mathrm{k}}\right)\right] .}
\end{aligned}
$$

Using the equations above, the Thomson scattered intensity can be written as

$$
\begin{aligned}
& \frac{\text { Flux }}{\mathrm{d} \omega \mathrm{dkd} \Omega}=\frac{\mathrm{v}_{\mathrm{gs}}\left\langle\mathrm{E}^{2}(\overrightarrow{\mathrm{x}}, \mathrm{t})\right\rangle}{8 \pi \mathrm{d} \omega \mathrm{dkd} \Omega}=\frac{\mathrm{v}_{\mathrm{gs}} \mathrm{n}_{\mathrm{oe}}}{8 \pi(2 \pi)^{3} 16 \omega_{\mathrm{o}}^{2}}\left(\frac{\omega_{\mathrm{pe}}^{2}}{\mathrm{n}_{\mathrm{oe}}}\right)^{2} \frac{\mathrm{E}_{\mathrm{o}}^{2}\left(\hat{\mathrm{e}}_{\mathrm{sc}} \cdot \hat{\mathrm{e}}_{\mathrm{pr}}\right)^{2}}{|\beta|^{2} \mathrm{k}} \\
& {\left[\left\{1-\frac{\chi_{\mathrm{e}}(\omega, \overrightarrow{\mathrm{k}})}{\varepsilon(\omega, \overrightarrow{\mathrm{k}})}\right\}^{2} \mathrm{f}_{\mathrm{oe}}\left(\frac{\omega}{\mathrm{k}}\right)+\left\{\frac{-\chi_{\mathrm{e}}(\omega, \overrightarrow{\mathrm{k}})}{\varepsilon(\omega, \overrightarrow{\mathrm{k}})}\right\}^{2} \sum_{\alpha} \mathrm{z}_{\alpha}^{2} \mathrm{f}_{\mathrm{oi} \alpha}\left(\frac{\omega}{\mathrm{k}}\right)\right] .}
\end{aligned}
$$




\section{Appendix 4.2}

\section{Derivation of the Thomson scattering form factor in a homogeneous plasma}

This appendix details the calculation of the Thomson scattered spectrum in a homogeneous plasma.

The detected Thomson scattered spectrum is proportional to the ensemble average of the product of the spectral scattered vector potential $\left\langle A_{s c}(\omega, \vec{k}) A_{s c}^{*}(\omega, \vec{k})\right)$. It was shown in Appendix 4.1 that $\left\langle A_{s c}(\omega, \vec{k}) A_{s c}^{*}(\omega, \vec{k})\right\rangle$ is proportional to $\left\langle\frac{n_{\text {epw }}}{n_{\mathrm{oe}}}\left(\omega+\omega_{\mathrm{pr}}, \overrightarrow{\mathrm{k}}+\overrightarrow{\mathrm{k}}_{\mathrm{pr}}\right) \frac{\mathrm{n}_{\mathrm{epw}}^{*}}{\mathrm{n}_{\mathrm{oe}}}\left(\omega+\omega_{\mathrm{pr}}, \overrightarrow{\mathrm{k}}+\overrightarrow{\mathrm{k}}_{\mathrm{pr}}\right)\right\rangle$. A detailed picture of the scattered spectrum, therefore, requires a kinetic calculation of the spectrum of electrostatic waves driven in the plasma. The kinetic calculation permits the treatment of the Landau damping of the ion acoustic waves.

The derivation of the electrostatic waves spectrum begins with the Vlasov equation, also known as the "correlationless" Boltzman equation. The Vlasov equation[91], 


$$
\frac{\partial f_{\alpha}}{\partial t}+\vec{v} \cdot \frac{\partial f_{\alpha}}{\partial \vec{x}}+\vec{a} \cdot \frac{\partial f_{\alpha}}{\partial \vec{v}}=0
$$

is very similar to the Boltzman equation from statistical mechanics, showing that the total derivative of the particle distribution function, $f_{\alpha}$, in phase space is constant, ignoring collisions. The acceleration, $\vec{a}$, comes from the Lorentz force law, $\vec{a}=\left(q / m_{e}\right)(\vec{E}+\vec{v} \times \vec{B} / c)$, and represents the average fields generated in the plasma, as well as external fields imposed on the plasma.

The Vlasov equation allows the determination of the velocity distribution for the particles in the plasma, as well as the collective oscillations. From this information, along with Maxwell's equations, the Thomson scattered spectrum can be derived. The first step is to linearize the Vlasov equation by assuming the particle distribution contains a zero-order component which is a function of velocity only, as well as a first-order part which is a function of position, time, and velocity. This leads to a set of linearized equations,

$$
\begin{aligned}
& \frac{\partial \mathrm{f}_{1 \mathrm{e}}}{\partial \mathrm{t}}+\overrightarrow{\mathrm{v}} \cdot \frac{\partial \mathrm{f}_{1 \mathrm{e}}}{\partial \overrightarrow{\mathrm{x}}}-\frac{\mathrm{e} \overrightarrow{\mathrm{E}}}{\mathrm{m}_{\mathrm{e}}} \cdot \frac{\partial \mathrm{f}_{0 \mathrm{e}}}{\partial \overrightarrow{\mathrm{v}}}=0 \text { and } \\
& \sum_{\alpha} \frac{\partial \mathrm{f}_{1 \mathrm{i} \alpha}}{\partial \mathrm{t}}+\overrightarrow{\mathrm{v}} \cdot \frac{\partial \mathrm{f}_{1 \mathrm{i} \alpha}}{\partial \overrightarrow{\mathrm{x}}}+\frac{\mathrm{Z}_{\alpha} \mathrm{e} \overrightarrow{\mathrm{E}}}{\mathrm{m}_{\mathrm{i} \alpha}} \cdot \frac{\partial \mathrm{f}_{0 \mathrm{i} \alpha}}{\partial \overrightarrow{\mathrm{v}}}=0,
\end{aligned}
$$

one for the electrons and one for each of the different ion species. These equations in conjunction with Gauss' law, 


$$
\nabla \cdot \overrightarrow{\mathrm{E}}_{\beta}=4 \pi \rho=4 \pi\left(-\mathrm{en}_{1 \mathrm{e}}+\sum_{\alpha} \mathrm{z}_{\alpha} \mathrm{en}_{1 \mathrm{i} \alpha}+\mathrm{q}_{\beta} \delta\left[\overrightarrow{\mathrm{x}}-\left(\overrightarrow{\mathrm{x}}_{\mathrm{o}}+\overrightarrow{\mathrm{v}}_{\mathrm{o}} \mathrm{t}\right)\right]\right)
$$

determine the electrostatic fluctuations in a magnetic field-free homogeneous plasma. The electric field, $\overrightarrow{\mathrm{E}}_{\beta}$, in Gauss' law above represents the electric field produced by the shielding of a given test particle in the plasma. The testparticle approach allows for the determination of the fluctuation level using each of the particles in the plasma as a test charge to find the fields generated by the shielding of the individual charges. These shielding fields are then added to the electric field produced by the charges themselves to obtain the total field generated in the plasma[89-93].

The electric field due to a test particle can be found by Fourier transforming Gauss's law along with the linearized version of Vlasov's equations. The electric field due to the shielding cloud surrounding a given test particle is then

$$
E_{\beta}(\omega, \vec{k})=\frac{-i}{k} \frac{4 \pi q_{\beta}}{(2 \pi)^{3}} \frac{e^{-i \vec{k} \cdot \vec{x}_{\circ}} \delta\left(\omega-\vec{k} \cdot \vec{v}_{o}\right)}{\varepsilon(\omega, \vec{k})}
$$

where $\varepsilon(\omega, \vec{k})=1+\frac{4 \pi e^{2}}{k^{2} m_{e}} \int \frac{\vec{k} \cdot \nabla_{v} f_{o e}}{(\omega-\vec{k} \cdot \vec{v})} d^{3} \vec{v}+\sum_{\alpha} \frac{4 \pi e^{2} z_{\alpha}^{2}}{k^{2} m_{\alpha}} \int \frac{\vec{k} \cdot \nabla_{v} f_{o i \alpha}}{(\omega-\vec{k} \cdot \vec{v})} d^{3} \vec{v} . \quad$ The electron density fluctuation is obtained by integrating the first-order electron distribution function, present in equation 4.2.2, over all velocity space. Taking into account the Fourier transform of the test electrons themselves, the spectral electron density fluctuation is 


$$
\begin{aligned}
\mathrm{n}_{1 \mathrm{e}}(\omega, \overrightarrow{\mathrm{k}}) & =\int \mathrm{f}_{1 \mathrm{e}}(\omega, \overrightarrow{\mathrm{k}}) \mathrm{d}^{3} \overrightarrow{\mathrm{v}}+\sum_{\text {electrons }} \text { particles } \\
& =\frac{\operatorname{ieE}(\omega, \overrightarrow{\mathrm{k}})}{|\mathrm{k}| \mathrm{m}_{\mathrm{e}}} \int \frac{\overrightarrow{\mathrm{k}} \cdot \nabla_{\mathrm{v}} \mathrm{f}_{\mathrm{oe}}}{(\omega-\overrightarrow{\mathrm{k}} \cdot \overrightarrow{\mathrm{v}})} \mathrm{d}^{3} \overrightarrow{\mathrm{v}}+\sum_{\text {electrons }} \frac{\mathrm{e}^{-\mathrm{i} \overrightarrow{\mathrm{k}} \cdot \overrightarrow{\mathrm{x}}_{\circ}} \delta\left(\omega-\overrightarrow{\mathrm{k}} \cdot \overrightarrow{\mathrm{v}}_{\mathrm{o}}\right)}{(2 \pi)^{3}}
\end{aligned}
$$

The electric field, $\mathrm{E}(\omega, \overrightarrow{\mathrm{k}})=\sum_{\text {electrons }} \mathrm{E}_{\beta}(\omega, \overrightarrow{\mathrm{k}})+\sum_{\text {species }} \sum_{\text {ions }} \mathrm{E}_{\beta}(\omega, \overrightarrow{\mathrm{k}})$, in equation 4.2.6 is composed of the shielding cloud fields produced from each of the individual test particles. Inserting the total shielding electric field calculated in 4.2.5 into 4.2 .6 shows that the spectral electron density fluctuation may be written as

$$
\begin{aligned}
\mathrm{n}_{1 \mathrm{e}}(\omega, \overrightarrow{\mathrm{k}})= & \left\{1-\frac{\chi_{\mathrm{e}}(\omega, \overrightarrow{\mathrm{k}})}{\varepsilon(\omega, \overrightarrow{\mathrm{k}})}\right\} \sum_{\text {electrons }} \frac{\mathrm{e}^{-\mathrm{i} \overrightarrow{\mathrm{k}} \cdot \overrightarrow{\mathrm{x}}_{\circ}} \delta\left(\omega-\overrightarrow{\mathrm{k}} \cdot \overrightarrow{\mathrm{v}}_{\mathrm{o}}\right)}{(2 \pi)^{3}}+ \\
& \left\{\frac{-\chi_{\mathrm{e}}(\omega, \overrightarrow{\mathrm{k}})}{\varepsilon(\omega, \overrightarrow{\mathrm{k}})}\right\} \sum_{\alpha} \sum_{\text {ions }} \frac{\mathrm{Z}_{\alpha} \mathrm{e}^{-\mathrm{i} \overrightarrow{\mathrm{k}} \cdot \overrightarrow{\mathrm{x}}_{\circ}} \delta\left(\omega-\overrightarrow{\mathrm{k}} \cdot \overrightarrow{\mathrm{v}}_{\mathrm{o}}\right)}{(2 \pi)^{3}}
\end{aligned}
$$

Assuming that the electrons and ions are uncorrelated and that the individual ion species are uncorrelated with each other, then all cross terms vanish and the

$$
\begin{gathered}
\left\langle\mathrm{n}_{1 \mathrm{e}}(\omega, \overrightarrow{\mathrm{k}}) \mathrm{n}_{1 \mathrm{e}}^{*}(\omega, \overrightarrow{\mathrm{k}})\right\rangle=\left\langle\left|1-\frac{\chi_{\mathrm{e}}(\omega, \overrightarrow{\mathrm{k}})}{\varepsilon(\omega, \overrightarrow{\mathrm{k}})}\right|^{2} \frac{1}{(2 \pi)^{6}}\left(\sum_{\text {electrons }} \delta\left(\omega-\overrightarrow{\mathrm{k}} \cdot \overrightarrow{\mathrm{v}}_{\mathrm{o}}\right)\right)^{2}+\right. \\
\left.\left|\frac{-\chi_{\mathrm{e}}(\omega, \overrightarrow{\mathrm{k}})}{\varepsilon(\omega, \overrightarrow{\mathrm{k}})}\right|^{2} \frac{1}{(2 \pi)^{6}} \sum_{\alpha}\left(\sum_{\text {ions }} \mathrm{z}_{\alpha} \delta\left(\omega-\overrightarrow{\mathrm{k}} \cdot \overrightarrow{\mathrm{v}}_{\mathrm{o}}\right)\right)^{2}\right\rangle
\end{gathered}
$$


The scattering form factor for a uniform plasma is typically defined as[92]

$$
\mathrm{s}(\overrightarrow{\mathrm{k}}, \omega)=\frac{(2 \pi)^{8}}{n_{\mathrm{e}} \mathrm{TV}}\left\langle\mathrm{n}_{1 \mathrm{e}}(\omega, \overrightarrow{\mathrm{k}}) \mathrm{n}_{1 \mathrm{e}}^{*}(\omega, \overrightarrow{\mathrm{k}})\right\rangle
$$

In equation 4.2.9 above, the square of the delta function can be interpreted as $\delta^{2}=\frac{T \delta}{(2 \pi)}$ and the ensemble average of the delta function can be interpreted as

$$
\left\langle\sum_{\text {particles }} \delta\left(\omega-\overrightarrow{\mathrm{k}} \cdot \overrightarrow{\mathrm{v}}_{\mathrm{o}}\right)\right\rangle=\left\langle\iint \mathrm{f}_{1 \alpha}(\overrightarrow{\mathrm{v}}) \mathrm{d}^{3} \overrightarrow{\mathrm{v}} \mathrm{d}^{3} \overrightarrow{\mathrm{x}}\right\rangle=\frac{\mathrm{V}}{\mathrm{k}} \mathrm{f}_{1 \alpha}(\omega / \mathrm{k})
$$

Therefore, the scattering form factor for a homogeneous plasma may be defined as[92]

$$
\mathrm{s}(\overrightarrow{\mathrm{k}}, \omega)=\frac{2 \pi}{n_{\mathrm{e}} \mathrm{k}}\left[\left|1-\frac{\chi_{\mathrm{e}}(\omega, \overrightarrow{\mathrm{k}})}{\varepsilon(\omega, \overrightarrow{\mathrm{k}})}\right|^{2} f_{1 \mathrm{e}}(\omega / \mathrm{k})+\left|\frac{-\chi_{\mathrm{e}}(\omega, \overrightarrow{\mathrm{k}})}{\varepsilon(\omega, \overrightarrow{\mathrm{k}})}\right|^{2} \sum_{\alpha} \mathrm{Z}_{\alpha}^{2} \mathrm{f}_{1 \mathrm{i} \alpha}(\omega / \mathrm{k})\right]
$$





\section{Appendix 4.3}

\section{Numerical calculation of Thomson scattering from an inhomogeneous plasma}

This appendix details the numerical calculation for the level of Thomson scattering produced from a fixed amplitude Langmuir wave located on the top of a parabolic profile. This is in fact applicable to many scattering processes present in inhomogeneous plasmas such as the generation of electromagnetic waves from the coupling of Langmuir waves, ion waves produced by the coupling of Langmuir waves, scattering of an electromagnetic wave from electrostatic waves present in the plasma(Thomson scattering), etc.

From Appendix 4.1, the equation describing the growth of the Thomson scattered vector potential is

$$
\begin{aligned}
& \left(\frac{\partial^{2}}{\partial t^{2}}+\omega_{p e}^{2}-c^{2} \nabla^{2}\right) \frac{q A_{\text {scatt }}}{m_{e} c^{2}}= \\
& -\frac{4 \pi n_{o e} e^{2}}{m_{e}}\left(\hat{e}_{\text {scatt }} \cdot \hat{e}_{\text {probe }}\right) \frac{n_{\text {epw }}}{n_{o e}} \frac{q A_{\text {probe }}}{m_{e} c^{2}},
\end{aligned}
$$


where $\hat{e}_{\text {scatt }}$ and $\hat{e}_{\text {probe }}$ are unit vectors in the direction of the scattered and probe vector potential respectively. This equation can be further simplified by writing the vector potentials and the Langmuir wave fluctuation as a slowly varying component multiplied by a rapid oscillation, or more precisely $\frac{\mathrm{qA}_{\alpha}}{\mathrm{m}_{\mathrm{e}} \mathrm{c}^{2}}=\frac{1}{2} \Psi_{\alpha}(\mathrm{x}, \mathrm{t}) \exp \left(\mathrm{i} \int \overrightarrow{\mathrm{k}}_{\alpha} \cdot \mathrm{d} \overrightarrow{\mathrm{x}}-\mathrm{i} \omega \mathrm{t}\right)+$ c.c.. $\quad$ The assumption that $\frac{\partial \Psi_{\alpha}(\mathrm{x}, \mathrm{t})}{\partial \mathrm{x}} \ll<\mathrm{k} \Psi_{\alpha}(\mathrm{x}, \mathrm{t})$ and $\frac{\partial \Psi_{\alpha}(\mathrm{x}, \mathrm{t})}{\partial \mathrm{t}} \ll \omega \Psi_{\alpha}(\mathrm{x}, \mathrm{t})$ reduces equation 4.3.1 to the first order partial differential equation

$$
\begin{aligned}
& \left(\frac{\partial}{\partial t}+\vec{v}_{\text {gscatt }} \cdot \frac{\partial}{\partial \vec{x}}\right) \Psi_{\text {scatt }}(x, t)= \\
& \frac{\omega_{\text {pe }}^{2} \mathrm{i}\left(\hat{\mathrm{e}}_{\text {scatt }} \cdot \hat{\mathrm{e}}_{\text {probe }}\right)}{4 \omega_{\mathrm{s}}}\left(\Psi_{\text {probe }} \Psi_{\text {epw }}\right) \exp \left[i K^{\prime \prime} x^{3} / 6\right]
\end{aligned}
$$

where the Taylor expanded phase mismatch on the top of the parabolic profile, $x_{0}$, is $k_{\text {probe }} \pm k_{\text {epw }}-k_{\text {scatt }}=\kappa(x) \approx \kappa^{\prime \prime}\left(x_{0}\right) x^{2} / 2$. Making the substitutions $\chi=\left(\kappa^{\prime \prime}\right)^{1 / 3} \mathrm{x}$ and $\tau=\frac{\omega_{\mathrm{pe}}^{2} \Psi_{\mathrm{probe}} \Psi_{\mathrm{epw}}}{\omega_{\mathrm{s}}} \mathrm{t}$ transforms equation 4.3.2 into

$$
\begin{aligned}
& \left(\frac{\partial}{\partial \tau}+\frac{\omega_{\mathrm{s}}\left(\kappa^{\prime \prime}\right)^{1 / 3} \mathrm{v}_{\text {gscatt }}}{\omega_{\mathrm{pe}}^{2} \Psi_{\text {probe }} \Psi_{\text {epw }}} \frac{\partial}{\partial \chi}\right) \Psi_{\text {scatt }}(\chi, \tau)= \\
& \frac{\mathrm{i}\left(\hat{\mathrm{e}}_{\text {scatt }} \cdot \hat{\mathrm{e}}_{\text {probe }}\right)}{4} \exp \left[\mathrm{i} \chi^{3} / 6\right] .
\end{aligned}
$$


The method of characteristics is used to numerically solve equation 4.3.3. Two new variables are introduced $\zeta=\chi+\frac{\omega_{\text {pe }}^{2} \Psi_{\text {probe }} \Psi_{\text {epw }}}{\omega_{s}\left(\kappa^{\prime \prime}\right)^{1 / 3} v_{\text {gscatt }}} \tau$ and $\eta=\chi-\frac{\omega_{\mathrm{pe}}^{2} \Psi_{\mathrm{probe}} \Psi_{\mathrm{epw}}}{\omega_{\mathrm{s}}\left(\kappa^{\prime \prime}\right)^{1 / 3} \mathrm{v}_{\mathrm{gscatt}}} \tau$. Upon substitution of the above new variables, equation 4.3.3 transforms into

$$
\begin{aligned}
& \frac{\partial \Psi_{\mathrm{s}}}{\partial \zeta}\left(\frac{\partial \zeta}{\partial \tau}+\frac{\omega_{\mathrm{s}}\left(\kappa^{\prime \prime}\right)^{1 / 3} \mathrm{v}_{\mathrm{gscatt}}}{\omega_{\mathrm{pe}}^{2} \Psi_{\text {probe }} \Psi_{\text {epw }}} \frac{\partial \zeta}{\partial \chi}\right)+ \\
& \frac{\partial \Psi_{\mathrm{s}}}{\partial \eta}\left(\frac{\partial \eta}{\partial \tau}+\frac{\omega_{\mathrm{s}}\left(\kappa^{\prime \prime}\right)^{1 / 3} \mathrm{v}_{\mathrm{gscatt}}}{\omega_{\mathrm{pe}}^{2} \Psi_{\text {probe }} \Psi_{\mathrm{epw}}} \frac{\partial \eta}{\partial \chi}\right)=\frac{\mathrm{i}\left(\hat{\mathrm{e}}_{\mathrm{scatt}} \cdot \hat{\mathrm{e}}_{\mathrm{probe}}\right)}{4} \exp \left[\mathrm{i} \chi^{3} / 6\right] .
\end{aligned}
$$

Equation 4.3 .4 can then be written as

$$
\frac{\partial \Psi_{\text {scatt }}}{\partial \zeta}=\frac{\mathrm{i}\left(\hat{\mathrm{e}}_{\text {scatt }} \cdot \hat{\mathrm{e}}_{\text {probe }}\right) \exp \left[\mathrm{i} \chi^{3} / 6\right]}{4\left[\frac{\omega_{\text {pe }}^{2} \Psi_{\text {probe }} \Psi_{\text {epw }}}{\omega_{\mathrm{s}}\left(\kappa^{\prime \prime}\right)^{1 / 3} \mathrm{v}_{\text {gscatt }}}+\frac{\omega_{\mathrm{s}}\left(\kappa^{\prime \prime}\right)^{1 / 3} \mathrm{v}_{\mathrm{gscatt}}}{\omega_{\mathrm{pe}}^{2} \Psi_{\text {probe }} \Psi_{\text {epw }}}\right]^{\prime}}
$$

where $\Delta \zeta=\Delta \tau\left[\frac{\omega_{\text {pe }}^{2} \Psi_{\text {probe }} \Psi_{\text {epw }}}{\omega_{s}\left(\kappa^{\prime \prime}\right)^{1 / 3} v_{\text {gscatt }}}+\frac{\omega_{s}\left(\kappa^{\prime \prime}\right)^{1 / 3} v_{\text {gscatt }}}{\omega_{\text {pe }}^{2} \Psi_{\text {probe }} \Psi_{\text {epw }}}\right]$ and $\Delta \eta=-\Delta \zeta$. Given the above two variable relations, the change in spatial scale can be expressed in terms of the change in time or $\Delta \chi=\Delta \tau \frac{\omega_{\mathrm{s}}\left(\kappa^{\prime \prime}\right)^{1 / 3} \mathrm{v}_{\mathrm{gscatt}}}{\omega_{\mathrm{pe}}^{2} \Psi_{\text {probe }} \Psi_{\mathrm{epw}}}$.

Figure A.4.3.1a shows the amplitude of the scattered vector potential from a plasma containing a quadratic phase mismatch. This situation is found when Thomson scattering from Langmuir waves located at the top of a 
parabolic profile. The initial conditions are that at $t=0$, the amplitude of the scattered vector potential is zero everywhere is space. In this figure, the
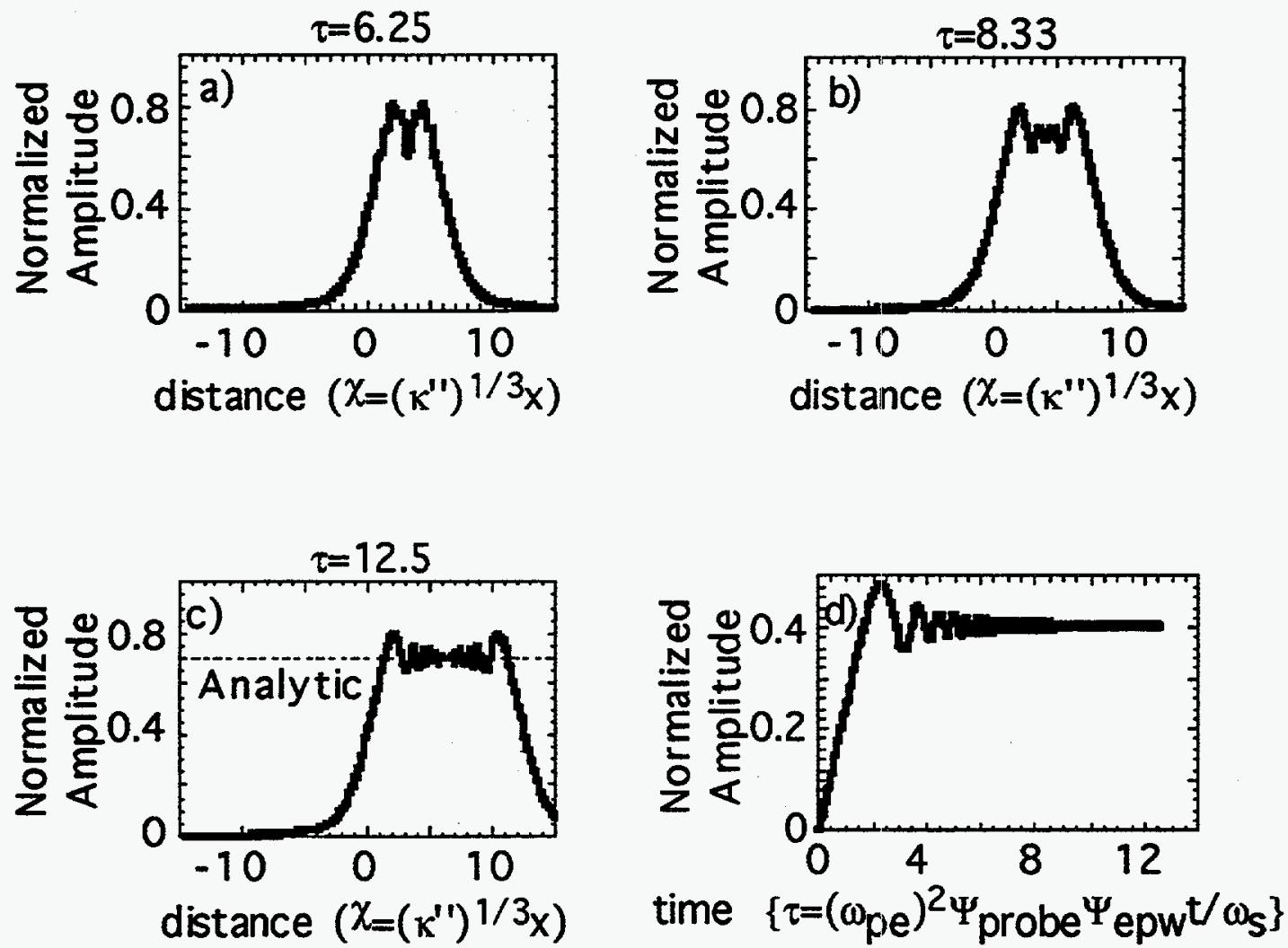

Figure A.4.3.1 Thomson scattered vector potential produced in a plasma with a quadratic phase mismatch between the interacting waves. The scattered vector potential as a function of distance is shown in figure A.4.3.1 a-c each representing a different time. The vertical axis on all of these graphs represents the amplitude of the Thomson scattered vector potential. The horizontal axis on figures a-c represent the distance along the profile with $\mathrm{x}=0$ representing the perfect phase matching point. The horizontal axis in figure A.4.3.1 d represents time. 
Thomson scattered signal has reached the saturation amplitude in figure A.4.3.1 $\mathrm{a}$ and the signal is traveling at the group velocity in figures A.4.3.1 b and A.4.3.1 c. The dashed line in figure A.4.3.1 c shows the amplitude of the steady state signal calculated in Chapter 5. Figure A.4.3.1 d shows the growth of the scattered vector potential at the perfect phase matching point. This graph shows the amplitude growing linearly in time at approximately the homogeneous growth rate, $\Psi_{\text {scatt }}=t \omega_{\text {pe }}^{2} \Psi_{\text {probe }} \Psi_{\text {epw }} / 4 \omega_{\mathrm{s}}$ with $\omega_{\text {pe }}^{2} \Psi_{\text {probe }} \Psi_{\text {epw }} / \omega_{\mathrm{s}}=1$ used in the simulation.

C This program will look at the amplitude of the Thomson

C scattered vector potential due to a parabolic phase mismatch.

$C$ This version uses method of characteristics.

PARAMETER (NSTEP=1200)

COMPLEX ScaNew(-NSTEP:NSTEP),ScaOld(-NSTEP:NSTEP),

!i,Disp(-NSTEP:NSTEP),DTs,ScaTime(0:NSTEP)

REAL ParDrive, Time, DT,DX

INTEGER NITER

open(unit=13,file='Expdet0',status='unknown')

close (unit $=13$,status ='delete')

open(unit $=13$,file $=$ 'Expdet0', status='unknown')

open(unit $=14$,file $=$ 'Expdet1',status='unknown')

close(unit=14,status='delete')

open(unit=14,file='Expdet1', status='unknown')

open(unit=15, file='Expdet2',status='unknown')

close(unit=15,status='delete')

open(unit $=15$,file $=$ 'Expdet2',status='unknown')

open (unit $=16$, file $=$ 'Expdet3', status='unknown')

close(unit=16,status='delete')

open(unit=16,file='Expdet3',status='unknown')

open(unit=17, file='Expdet4',status='unknown')

close (unit=17,status='delete')

open(unit=17, file='Expdet4',status='unknown')

open(unit=18,file='Exptime',status='unknown')

close(unit=18,status='delete')

open(unit=18,file='Exptime',status='unknown')

$\mathrm{i}=\operatorname{CMPL} X(0 ., 1$. 
10 PRINT *, 'Enter THE TOTAL NUMBER OF TIME STEPS (0 TO STOP)'

12 PRINT *, 'SUGGEST 1000.'

READ *,NTTER

IF (NITER .EQ. 0.) STOP

14 PRINT *, 'ENTER parabolic driving term'

16 PRINT *, 'SUGGEST 1.0'

READ *, ParDrive

$\mathrm{DT}=0.0125$

DX $=\mathrm{DT} /$ ParDrive

$C$ setup initial wave amplitudes

DO 20 IX=-NSTEP,NSTEP

ScaOld(IX) $=$ CMPLX $(0.000,0$.

ScaNew $($ IX $)=$ CMPLX $(0.000,0$.)

$\operatorname{Disp}(\mathrm{IX})=\mathrm{i}^{*}\left(\operatorname{REAL}\left(\mathrm{DX} \mathrm{I}^{*} \mathrm{IX}\right)\right)^{* * 3.0 / 6}$.

20 CONTINUE

$C$ end setup initial wave amplitudes

Time $=0$.

$C$ time loop

$\mathrm{DTs}=\mathrm{CMPLX}\left(\mathrm{DT}^{*} 0.25,0.0\right)$

$C$ take care of boundary conditions

ScaNew $(-N S T E P)=$ CMPLX $(0.000,0$.

$C$ end boundary conditions

DO 40 ITER=1, NITER

Time $=$ Time + DT

ScaTime(ITER) $=$ ScaOld $(0)$

$C$ fill out amplitude arrays at new time step

DO 50 IX=-NSTEP+1, NSTEP

ScaNew $(\mathrm{IX})=$ ScaOld(IX-1)-

! $\mathrm{i}^{*} \mathrm{DTs} \mathrm{s}^{*} \mathrm{CEXP}(\mathrm{Disp}(\mathrm{IX}-1)$ )

50 CONTINUE

DO $60 \mathrm{JX}=-\mathrm{NSTEP}$, NSTEP

ScaOld(JX) $=$ ScaNew(JX)

60 CONTINUE

IF (ITER.EQ. 3) THEN write $\left(13,{ }^{*}\right)$, 'Mag. of As',',','position'

DO $70 \mathrm{JX}=$-NSTEP, NSTEP, 4 write $(13, *), \mathrm{CABS}($ ScaOld(JX)),, ,

! JX*DX

70 Continue

close(unit=13)

Else IF (ITER.EQ.NITER/2) THEN write $\left(14,{ }^{*}\right)$, 'Mag. of As', ',',position' DO 75 JX=-NSTEP, NSTEP, 4 


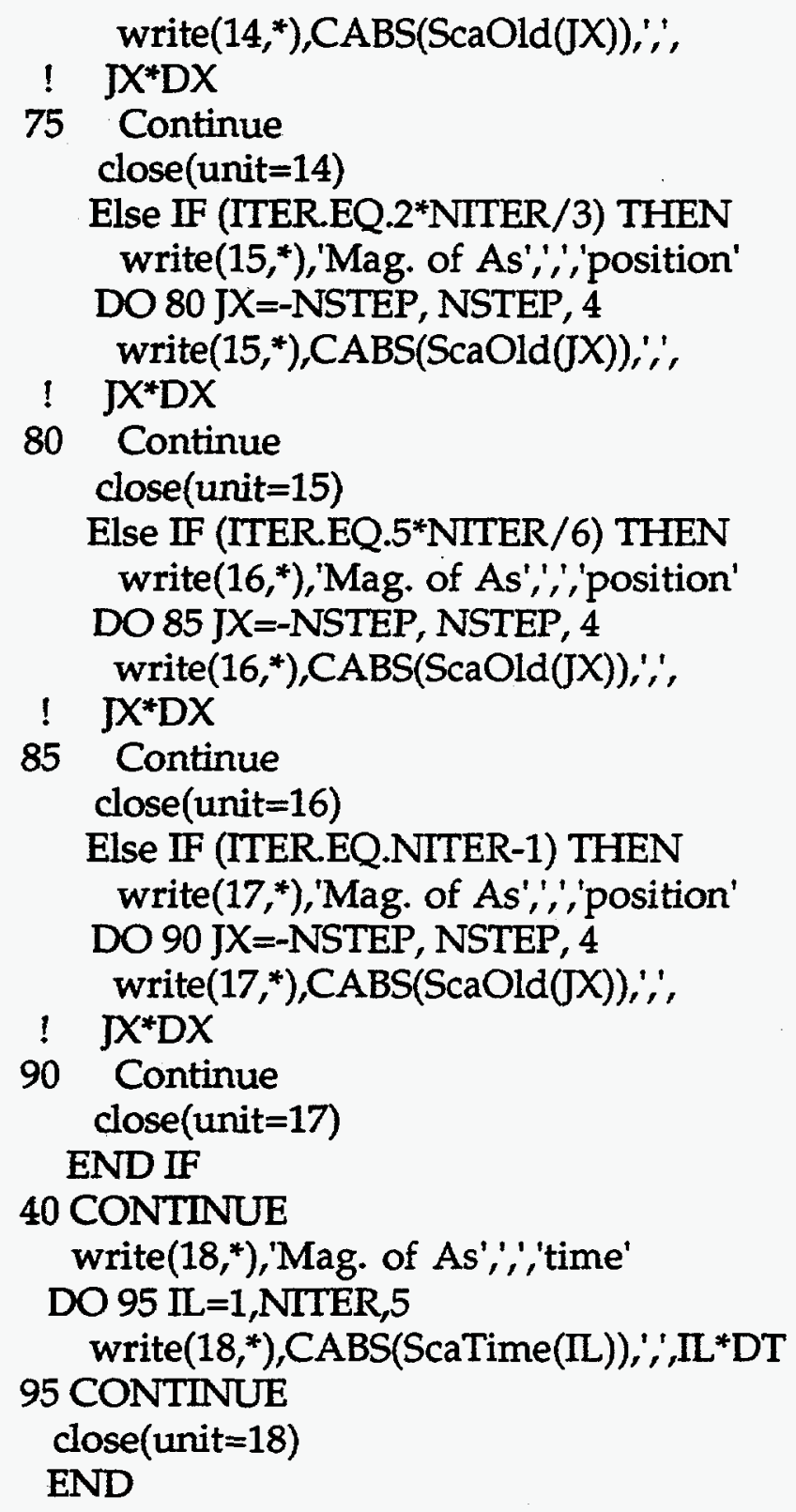





\section{Appendix 4.4}

\section{Graphical solution of the roots of the dispersion relation corresponding to ion acoustic waves}

This Appendix contains plots showing the roots corresponding to the solution to the dispersion relation $\varepsilon(\omega, \overrightarrow{\mathrm{k}})=1+\chi_{\mathrm{e}}(\omega, \overrightarrow{\mathrm{k}})+\sum_{\alpha} \chi_{\mathrm{i} \alpha}(\omega, \overrightarrow{\mathrm{k}})=0$ for the plasmas used in the experiments performed for this dissertation. In Figures A.4.4.1-A.4.4.3, the solid contours represent the zero contours of the $\operatorname{Im}\{\varepsilon(\omega, k)\}=0$ and the dashed lines represent the zero contours of the $\operatorname{Re}\{\varepsilon(\omega, \mathrm{k})\}=0$. The roots of the dispersion relation are then given by the intersection between the real and imaginary zero contours. Figure A.4.4.4 a-d shows the damping of the ion wave as a function of the ratio of ion temperature to electron temperature for the four different ion-species plasmas used in the experiments performed for this dissertation. 

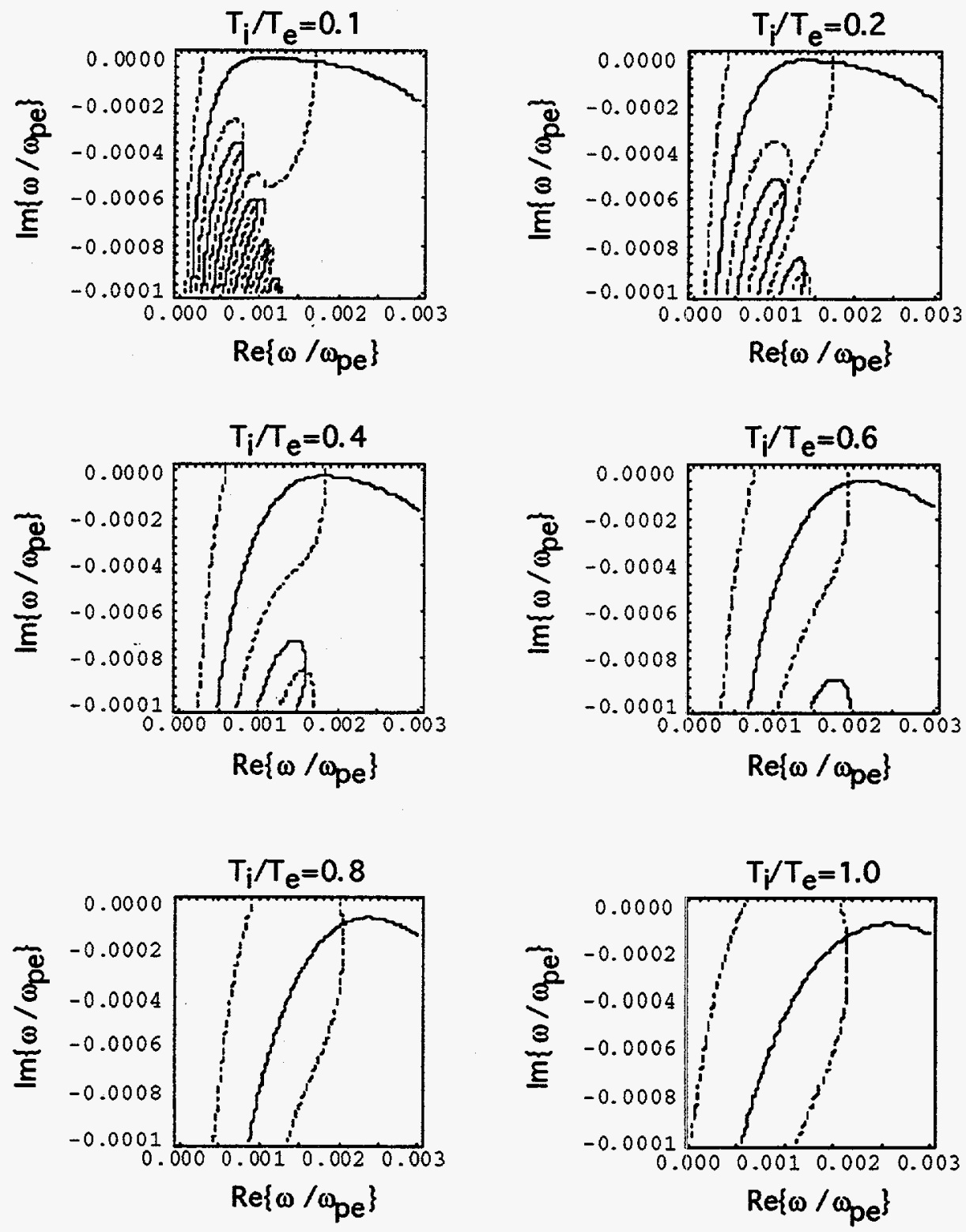

Figure A.4.4.1 Graphical solution of the roots of the dispersion relation in a Carbon plasma. Each figure represents the solution at a different ratio of ion to electron temperature. The vertical axes represent the imaginary component of the frequency while the horizontal axis represents the real component of the frequency. 

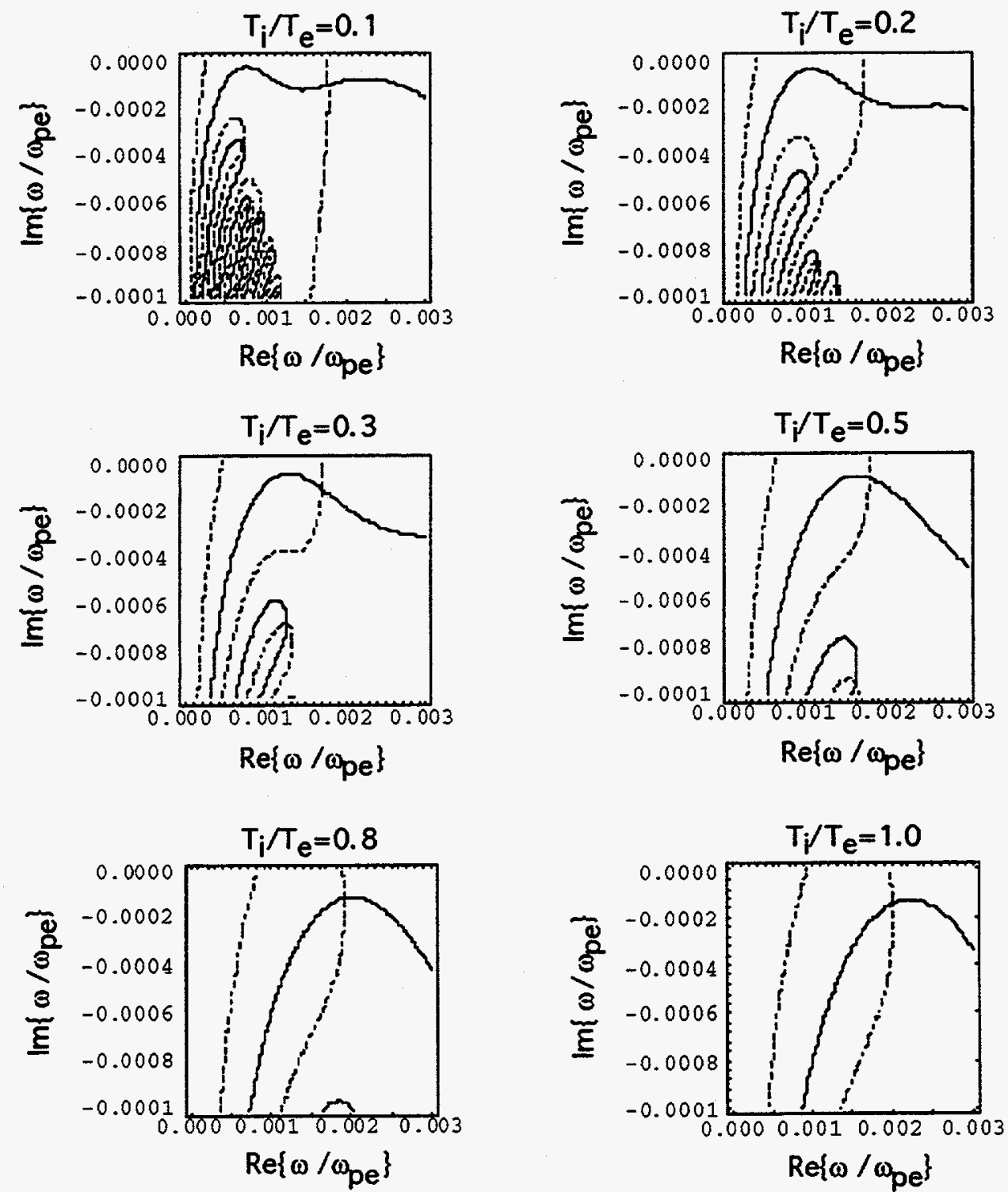

Figure A.4.4.2 Graphical solution of the roots of the dispersion relation in a Collodium plasma. Each figure represents the solution at a different ratio of ion to electron temperature. The vertical axes represent the imaginary component of the frequency while the horizontal axis represents the real component of the frequency. 

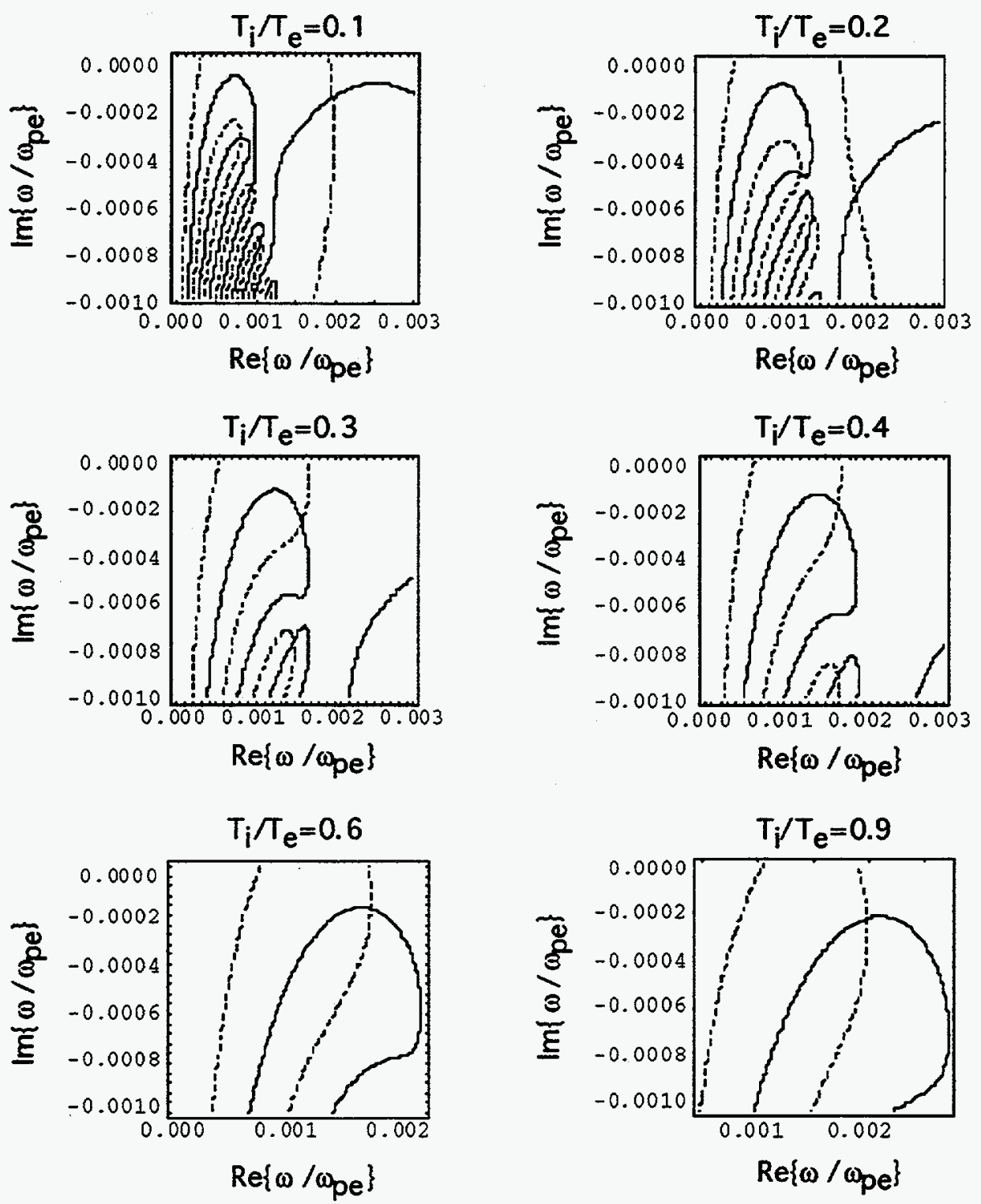

Figure A.4.4.3 Graphical solution of the roots of the dispersion relation in a $\mathrm{CH}$ plasma. Each figure represents the solution at a different ratio of ion to electron temperature. The vertical axes represent the imaginary component of the frequency while the horizontal axis represents the real component of the frequency. 

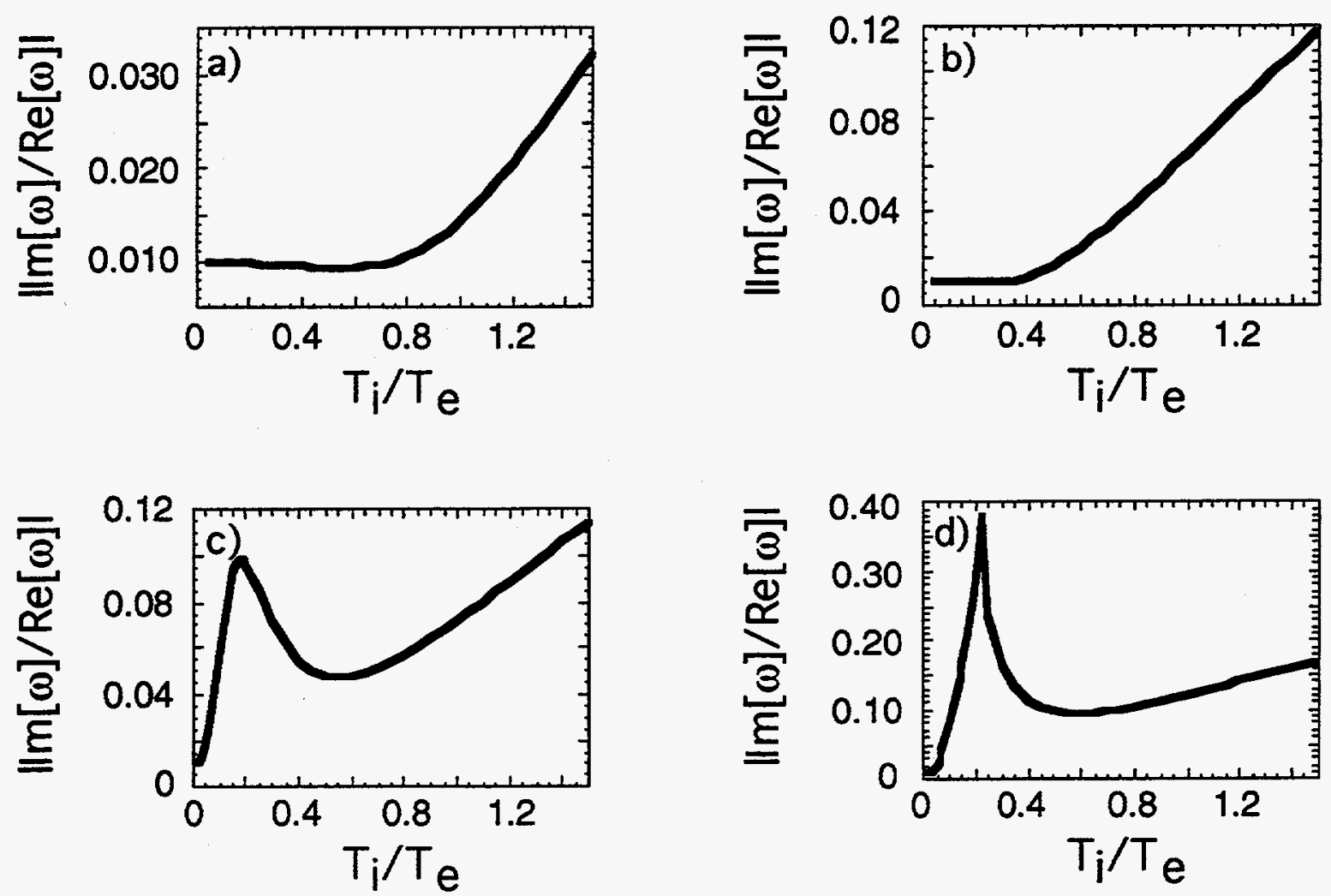

Figure A.4.4.4 Absolute magnitude of the ion wave damping normalized to the real part of the ion acoustic frequency for four different ion species plasmas. Each of these graphs were generated assuming $k \lambda_{D e}=0.1$ and that the ions were completely ionized. Figure 4.4.4 a-d represent the damping as a function of the ratio of ion temperature, $T_{i}$, to electron temperature, $T_{e}$, for an Aluminum plasma, a Carbon plasma, a Collodium plasma, and a $\mathrm{CH}$ plasma, respectively. 



\section{Appendix 4.5}

\section{Contour integrations for Thomson scattering}

The equation describing Thomson scattering from an inhomogeneous plasma with a linear phase mismatch between the waves is given by

$$
\frac{\mathrm{d} \Psi_{\mathrm{sc}}}{\mathrm{dx}}=\frac{\omega_{\mathrm{pe}}^{2}\left(\hat{\mathrm{e}}_{\mathrm{pr}} \cdot \hat{\mathrm{e}}_{\mathrm{sc}}\right)}{4 \mathrm{ic}^{2} \mathrm{k}_{\mathrm{sc}}}\left(\Psi_{\mathrm{epw}} \Psi_{\mathrm{pr}}\right) \exp \left[\frac{\mathrm{i} \kappa^{\prime} x^{2}}{2}\right]
$$

This equation can be written as

$$
\Psi_{\mathrm{sc}}(\infty)=\frac{\omega_{\mathrm{pe}}^{2}\left(\hat{\mathrm{e}}_{\mathrm{pr}} \cdot \hat{\mathrm{e}}_{\mathrm{sc}}\right)}{4 \mathrm{ic}^{2} \mathrm{k}_{\mathrm{sc}}} \sqrt{\frac{2}{\kappa^{\prime}}}\left(\Psi_{\mathrm{epw}} \Psi_{\mathrm{pr}}\right) 2 \int_{0}^{\infty} \exp \left[i m^{2}\right] d m
$$

The integral in equation 4.5.2 can be solved using the contour shown below in figure A.4.5.1 


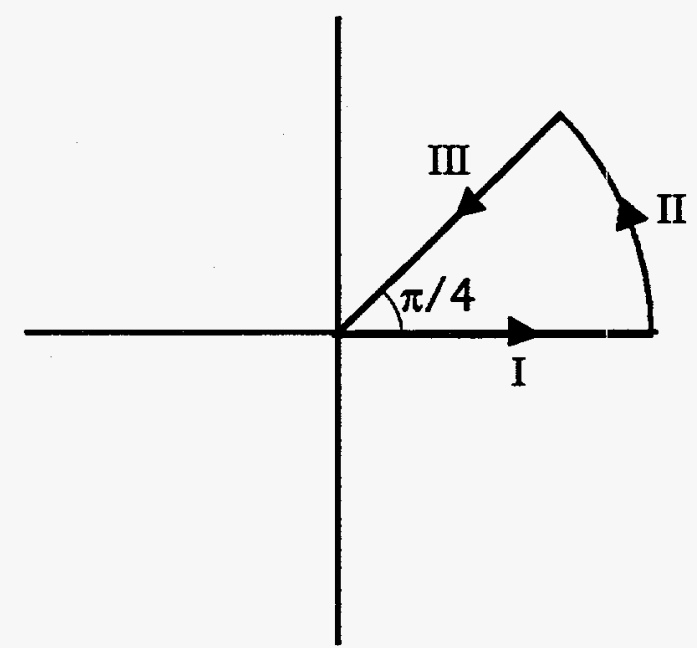

Figure A.4.5.1 Contour used to evaluate $\int \exp \left(\mathrm{im}^{2}\right) \mathrm{dm}$.

The contour is equal to

$$
\oint=\int_{\mathrm{I}}+\int_{\mathrm{II}}+\int_{\mathrm{II}}=2 \pi \mathrm{i} \sum \text { residues }=0,
$$

as labeled in figure 4.5.1. These integrals may be written as

$$
\begin{aligned}
& 0=\int_{0}^{\infty} \exp \left[i x^{2}\right] d x+\frac{\lim i t}{R \Rightarrow \infty} \int_{0}^{\pi / 4} \exp \left[i R^{2} \exp (i 2 \theta)\right] i \operatorname{Re} \exp (i \theta) d \theta \\
& +\int_{\infty}^{0} \exp \left[-r^{2}\right] \exp (i \pi / 4) d r .
\end{aligned}
$$

The integral is then equal to

$$
\int_{0}^{\infty} \exp \left[\mathrm{ix} \mathrm{x}^{2}\right] \mathrm{dx}=\exp (\mathrm{i} \pi / 4) \int_{0}^{\infty} \exp \left[-\mathrm{r}^{2}\right] \mathrm{dr}=\exp (\mathrm{i} \pi / 4) \sqrt{\pi} / 2
$$


The absolute magnitude of the Thomson scattered vector potential, with a linear phase mismatch between the interacting waves, is

$$
\left|\Psi_{s c}(\infty)\right|=\frac{\omega_{\mathrm{pe}}^{2}\left(\hat{\mathrm{e}}_{\mathrm{pr}} \cdot \hat{\mathrm{e}}_{\mathrm{sc}}\right)}{4 \mathrm{c}^{2} \mathrm{k}_{\mathrm{sc}}} \sqrt{\frac{2 \pi}{\kappa^{\prime}}}\left|\Psi_{\mathrm{epw}}\right| \Psi_{\mathrm{pr}} \mid
$$

The equation describing Thomson scattering from an inhomogeneous plasma with a quadratic phase mismatch between the waves is given by

$$
\frac{\mathrm{d} \Psi_{\mathrm{sc}}}{\mathrm{dx}}=\frac{\omega_{\mathrm{pe}}^{2}\left(\hat{\mathrm{e}}_{\mathrm{pr}} \cdot \hat{\mathrm{e}}_{\mathrm{sc}}\right)}{4 \mathrm{ic}^{2} \mathrm{k}_{\mathrm{sc}}}\left(\Psi_{\mathrm{epw}} \Psi_{\mathrm{pr}}\right) \exp \left[\frac{\mathrm{i} \kappa^{\prime \prime} x^{3}}{6}\right]
$$

This equation can be written as

$$
\Psi_{\mathrm{sc}}(\infty)=\frac{\omega_{\mathrm{pe}}^{2}\left(\hat{\mathrm{e}}_{\mathrm{pr}} \cdot \hat{\mathrm{e}}_{\mathrm{sc}}\right)}{4 \mathrm{ic}^{2} \mathrm{k}_{\mathrm{sc}}}\left(\frac{6}{\kappa^{\prime \prime}}\right)^{1 / 3}\left(\Psi_{\mathrm{epw}} \Psi_{\mathrm{pr}}\right) \int_{-\infty}^{\infty} \exp \left[i m^{3}\right] d m
$$

The integral in equation 4.5 .8 can be solved using the contours shown below in figure A.4.5.2 


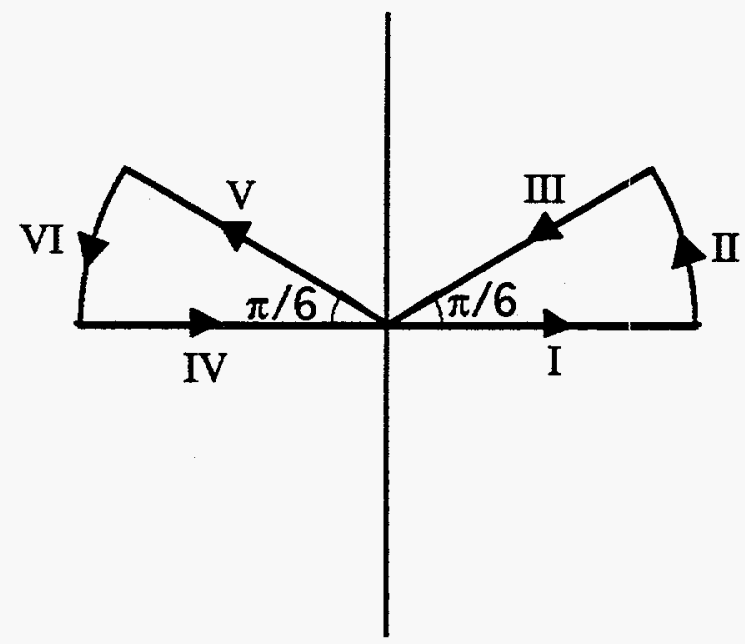

Figure A.4.5.2 Contours used to evaluate $\int \exp \left(\mathrm{im}^{3}\right) \mathrm{dm}$.

The contours shown in figure A.4.5.2 can be written as

$$
\begin{aligned}
& \oint_{\mathrm{a}}=\int_{\mathrm{I}}+\int_{\mathrm{II}}+\int_{\mathrm{II}}=2 \pi \mathrm{i} \sum \text { residues }=0 \text { and } \\
& \oint_{\mathrm{b}}=\int_{\mathrm{IV}}+\int_{\mathrm{V}}+\int_{\mathrm{VI}}=2 \pi \mathrm{i} \sum \text { residues }=0 .
\end{aligned}
$$

These contours may be written as

$$
\begin{aligned}
& \oint_{a}=0=\int_{0}^{\infty} \exp \left[i x^{3}\right] d x+\frac{\lim i t}{R \Rightarrow \infty} \int_{0}^{\pi / 6} \exp \left[i R^{3} \exp (i 3 \theta)\right] i \operatorname{Rexp}(i \theta) d \theta \\
& +\int_{\infty}^{0} \exp \left[-r^{3}\right] \exp (i \pi / 6) d r \text { and }
\end{aligned}
$$




$$
\begin{aligned}
& \oint_{b}=0=\int_{-\infty}^{0} \exp \left[i x^{3}\right] d x+\int_{0}^{\infty} \exp \left[-r^{3}\right] \exp (i 5 \pi / 6) d r \\
& +\frac{\lim i t}{R \Rightarrow \infty} \int_{5 \pi / 6}^{\pi} \exp \left[i R^{3} \exp (i 3 \theta)\right] i \operatorname{Rexp}(i \theta) d \theta
\end{aligned}
$$

Equations 4.5.11 and 4.5.12 reduce to

$$
\begin{aligned}
& \int_{0}^{\infty} \exp \left[\mathrm{ix} \mathrm{x}^{3}\right] \mathrm{dx}=\exp (\mathrm{i} \pi / 6) \int_{0}^{\infty} \exp \left[-\mathrm{r}^{3}\right] \mathrm{dr} \text { and } \\
& \int_{-\infty}^{0} \exp \left[\mathrm{ix}{ }^{3}\right] \mathrm{dx}=-\exp (\mathrm{i} 5 \pi / 6) \int_{0}^{\infty} \exp \left[-\mathrm{r}^{3}\right] \mathrm{dr}
\end{aligned}
$$

respectively. The original integral of interest is then equal to

$$
\begin{aligned}
& \int_{-\infty}^{\infty} \exp \left[i x^{3}\right] d x=[\exp (i \pi / 6)-\exp (i 5 \pi / 6)] \int_{0}^{\infty} \exp \left[-r^{3}\right] d r= \\
& \sqrt{3} \int_{0}^{\infty} \exp \left[-r^{3}\right] d r
\end{aligned}
$$

The remaining integral can be written in terms of a Gamma function, $\Gamma$,

$$
\int_{0}^{\infty} \exp \left[-\mathrm{r}^{3}\right] \mathrm{dr}=(3)^{-1} \int_{0}^{\infty}(J)^{-2 / 3} \exp [-\mathrm{J}] \mathrm{dJ}=\Gamma(1 / 3) / 3
$$

The absolute magnitude of the Thomson scattered vector potential, with a quadratic phase mismatch between the interacting waves, is 


$$
\left|\Psi_{\mathrm{sc}}(\infty)\right|=\frac{\omega_{\mathrm{pe}}^{2}\left(\hat{\mathrm{e}}_{\mathrm{pr}} \cdot \hat{\mathrm{e}}_{\mathrm{sc}}\right)}{4 \mathrm{c}^{2} \mathrm{k}_{\mathrm{sc}}}\left(\frac{6}{\kappa^{\prime \prime}}\right)^{1 / 3} \frac{\Gamma(1 / 3)}{\sqrt{3}}\left|\Psi_{\mathrm{epw}}\right| \Psi_{\mathrm{pr}} \mid
$$




\section{Bibliography}

1. P. Amendt, D.C. Eder, and S.C. Wilks, "X-ray Lasing by Optical-FieldInduced Ionization," Phys. Rev. Lett. 66, 2589 (1991).

2. C. Joshi, W.B. Mori, T. Katsouleas, J.M. Dawson, J.M. Kindel, and D.W. Forslund, "Ultrahigh gradient particle acceleration by intense laser-driven plasma density waves," Nature 311, 525 (1984).

3. F. Amiranoff, M. Laberge, J.R. Marques, F. Moulin, E. Fabre, B. Cros, G. Matthieussent, P. Benkheiri, F. Jacquet, J. Meyer, Ph. Mine, C. Stenz, and P. Mora, "Observation of Modulational Instability in Nd-Laser Beat-Wave Experiments," Phys. Rev. Lett. 68, 3710 (1992).

4. C. Darrow, W. B. Mori, T. Katsouleas, C. Joshi, D. Umstader, and C. E. Clayton, "Electrostatic Mode Coupling of Beat-Excited Electron Plasma Waves," IEEE Trans. on Plasma Science PS-15, 107 (1987). 
5. J.H. Rogers, D.Q. Hwang, J.C. Thomas, R.L. Horton, J. Killeen, and G. Dimonte, "Beat wave excitation of electron plasma waves in a toroidal magnetized plasma," Phys. Fluids B 4, 1920 (1992).

6. B.I. Cohen, R.H. Cohen, B.G. Logan, W. McCay Nevins, G.R. Smith, A.V. Kluge, and A.H. Kritz, "Beat Wave Current Drive with Intense Pulsed Free-Electron Lasers," Nuclear Fusion 28, 1519 (1988).

7. M.N. Rosenbluth and C.S. Liu, "Excitation of plasma waves by two laser beam," Phys. Rev. Lett. 29, 701 (1972).

8. P. Bertrand, A. Ghizzo, S.J. Karttunen, T.J.H. Pattikangas, R.R.E. Salomaa, and M. Shoucri, "Two-stage electron acceleration by simultaneous stimulated Raman backward and forward scattering," Phys. of Plasmas 2, 3115 (1995).

9. T. Kolber, W. Rozmus, and V.T. Tikhonchuk, "Saturation of stimulated Raman scattering by Langmuir and ion-acoustic wave coupling," Phys. Fluids B 5, 138 (1993).

10. D. F. Dubois, D. A. Russel, and Harvey A. Rose, "Saturation Spectra of the Two-Plasmon Decay instability," Phys. Rev. Lett. 74, 3983 (1995). 
11. H. A. Rose, D. F. DuBois, and B. Bezzerides, "Nonlinear coupling of stimulated Raman and Brillouin scattering in laser-plasma interactions," Phys. Rev. Lett. 58, 2547 (1987).

12. Guy Bonnaud, Denis Pesme, and Rene Pellat, "Nonlinear Raman scattering behavior with Langmuir and sound waves coupling in a homogeneous plasma," Phys. Fluids B 2, 1618 (1990).

13. B. Bezzerides, D.F. DuBois, and H.A. Rose, "Saturation of Stimulated Raman scattering by the excitation of strong Langmuir turbulence," Phys. Rev. Lett. 70, 2569 (1993).

14. Francis F. Chen, Introduction to Plasma Physics and Controlled Fusion (Plenum Press, New York, 1984).

15. Dwight R. Nicholson, Introduction to Plasma Theory (John Wiley \& Sons, New York, 1983).

16. William L. Kruer, The Physics of Laser Plasma Interactions (AddisonWesley Publishing Company, Inc., Redwood City, California, 1988).

17. H. A. Baldis, E. M. Campbell, and W. L. Kruer, "Laser plasma Interactions," in Handbook of Plasma Physics, edited by A. Rubenchik and S. Witkowski (North-Holland, Amsterdam, 1991), pp. 404. 
18. Alan C. Newell and Jerome V. Moloney, Nonlinear Optics (AddisonWesley Publishing Company, Redwood City, 1992).

19. C. E. Max, Theory of the Coronal Plasma in Laser Fusion Targets (Lawrence Livermore Laboratory, University of California, Livermore, California, 1981).

20. R. P. Drake, H. A. Baldis, R. L. Berger, W. L. Kruer, E. A. Williams, Kent Estabrook, T. W. Johnston, and P. E. Young, "Observation of Stimulated Compton Scattering from Resonant Electrons in a Laser-Produced Plasma," Phys. Rev. Lett. 64, 423 (1990).

21. A. Simon, W. Seka, L. M. Goldman, and R. W. Short, "Raman scattering in inhomogeneous laser-produced plasma," Phys. Fluids 29, 1704 (1986).

22. R. P. Drake, E. A. Williams, P. E. Young, Kent Estabrook, W. L. Kruer, H. A. Baldis, and T. W. Johnston, "Reduction of emission in the stimulated Raman scattering frequency band to thermal noise levels by collisional damping in a laser-produced plasma," Phys. Rev. A 39, 3536 (1989).

23. R. E. Turner, Kent Estabrook, R. L. Kauffman, D. R. Bach, R. P. Drake, D. W. Phillion, B. F. Lasinski, W. L. Kruer, E. A. Williams, and E. M. Campbell, "Evidence for Collisional Damping in High-Energy Raman Scattering Experiments at 0.26 Microns," Phys. Rev. Lett. 54, 189 (1985). 
24. R. P. Drake, E. A. Williams, P. E. Young, Kent Estabrook, W. L. Kruer, and D. S. Montgomery, "Narrow Raman spectra: The competition between collisional and Landau damping," Phys. Fluids B 1, 2217 (1989).

25. D. R. Nicholson, "Parametric instabilities in plasma with sinusoidal density modulation," Phys. Fluids 19, 889 (1976).

26. K. Nishikawa and C. S. Liu, "General Formalism of Parametric Excitation," in Advances in Plasma Physics, edited by A. Simon and W. Thompson (Interscience, New York, 1976), pp. 1.

27. Marshall N. Rosenbluth, "Parametric Instabilities in Inhomogeneous Media," Phys. Rev. Lett. 29, 565 (1972).

28. R. White, P. Kaw, D. Pesme, M. N. Rosenbluth, G. Laval, R. Huff, and R. Varma, "Absolute parametric instabilities in inhomogeneous plasmas," Nucl. Fusion 24, 45 (1974).

29. E. A. Williams, in Laser Program Annual Report 85 (Lawrence Livermore National Laboratory, Livermore, CA, 1985), pp. 2.

30. S. H. Batha, D. S. Montgomery, K. S. Bradley, R. P. Drake, Kent Estabrook, and B. A. Remington, "Intensity Scaling of Stimulated Raman 
Forward Scattering in Laser-Produced Plasmas," Phys. Rev. Lett. 66, 2324 (1991).

31. C. E. Clayton, C.Joshi, and F.F.Chen, "Ion-Trapping Saturation of the Brillouin Instability," Phys. Rev. Lett. 51, 1656 (1983).

32. P. Koch and E. A. Williams, "Absolute growth of coupled forward and backward Raman scattering in inhomogeneous plasma," Phys. Fluids 27, 2346 (1984).

33. H. C. Barr, T. J. M. Boyd, and G. A. Coutts, "Nonlocal Effects and the Raman Instability," Phys. Rev. Lett. 60, 1950 (1988).

34. S. H. Batha, K. S. Bradley, R. P. Drake, Kent Estabrook, W.L. Kruer, D. S. Montgomery, and B. A. Remington, "Intensity Scaling and Saturation of Stimulated Raman Forward Scattering," Phys. Plasmas 1, 1985 (1994).

35. R. L. Berger, E. A. Williams, and A. Simon, "Effect of plasma noise spectrum on stimulated scattering in inhomogeneous plasma," Phys. Fluids B 1, 414 (1989).

36. E. A. Williams, Bull. Am. Phys. Soc. 37, 1375 (1992).

37. C. Rousseaux, G. Malka, J.L. Miquel, F. Amiranoff, S.D. Baton, and $\mathrm{Ph}$. Mounaix, "Experimental Validation of the Linear Theory of Stimulated 
Raman Scattering Driven by a 500-fs Laser Pulse in a Preformed Plasma," Phys. Rev. Lett. 74, 4655 (1995).

38. W.L. Kruer, "Interaction physics for megajoule laser fusion targets," in Laser Interaction and Related Plasma Phenomena, edited by G. H. Miley (Plenum Press, New York, 1991), pp. 503.

39. R.P. Drake and S.H. Batha, "The influence of subsidiary Langmuir decay on the spectrum of stimulated Raman scattering," Phys. Fluids B 3, 2936 (1991).

40. D.M. Villeneuve, K.L. Baker, R.P. Drake, B. Sleaford, and B. La Fontaine, "Observation of Plasma Waves by Thomson Scattering: Saturation of Stimulated Raman Scattering," Phys. Rev. Lett. 71, 368 (1993).

41. P.K. Shukla, M.Y. Yu, M. Mohan, R.K. Varma, and K.H. Spatschek, "Electromagnetic wave generation in a beam-plasma system," Phys. Rev. A 27, 552 (1983).

42. R.P. Lin, W.K. Levedahl, W. Lotko, D.A. Gurnett, and F.L. Scarf, "Evidence for nonlinear wave-wave interactions in solar type III radio bursts," Ap. J. 308, 954 (1986).

43. D.B. Melrose and M.V. Goldman, "Microstructures in Type III Events in the Solar Wind," Solar Physics 107, 329 (1987). 
44. T.B. Leyser, "Electromagnetic radiation by parametric decay of upper hybrid waves in ionospheric modification experiments," Phys. Plasmas 1, 2003 (1994).

45. S.L. Musher, A.M. Rubenchik, and V.E. Zakharo, "Weak Langmuir Turbulence," Physics Reports section 6.6 (1994).

46. K.L. Baker, B.B. Afeyan, K.G. Estabrook, R.P. Drake, and K.S. Bradley, "Langmuir-pumped plasmon-phonon decay: possible saturation mechanism for Stimulated Raman Scattering, Two Plasmon Decay, and Plasmon-Phonon Decay," presented at the Anomalous Absorption conference, 1994), p.

47. A. M. Rubenchik and V. E. Zakharov, "Strong Langmuir turbulence in laser plasma," in Physics of Laser Plasma, edited by A. Rubenchik and S. Witkowski (North-Holland, Amsterdam, 1991), pp. 335.

48. M. V. Goldman, "Strong turbulence of plasma waves," Rev. Mod. Phys. 56,709 (1984).

49. V.D. Shapiro and V.I. Shevchenko, "Strong turbulence of plasma oscillations," in Handbook of Plasma Physics, edited by A. A. Galeev and R. N. Sudan (North-Holland, Amsterdam, 1984), pp. 127. 
50. V.E. Zakharov, "Zak. eqs," Sov.Phys. - JETP 35, 908 (1972).

51. F. Moulin, F. Amiranoff, M. Laberge, J.R. Marques, B. Cros, G. Matthieussent, D. Bernard, F. Jacquet, Ph. Mine, A. Specka, C. Stenz, and P. Mora, "Coupling between electron and ion waves in Nd-laser beat-wave experiments," Phys. Plasmas 1, 1318 (1994).

52. V.E. Zakharov, "Collapse and self-focusing of Langmuir waves," in Handbook of Plasma Physics, edited by A. A. Galeev and R. N. Sudan (NorthHolland, Amsterdam, 1984), pp. 81.

53. A. Bruce Langdon, Barbara F. Lasinski, and William L. Kruer, "Nonlinear saturation and recurrence of the two-plasmon decay instability," Phys. Rev. Lett. 43, 133 (1979).

54. H. A. Baldis, D. M. Villeneuve, and C. J. Walsh, "Plasma waves in laser fusion plasmas," Can. J. Phys 64, 961 (1986).

55. C. L. Shepard, J. A. Tarvin, R. L. Berger, Gar E.Busch, R. R. Johnson, and R. J. Schroeder, "Raman scattering in experiments with planar Au targets irradiated with $0.53 \mu \mathrm{m}$ laser light," Phys. Fluids 29, 583 (1986).

56. D. M. Villeneuve, H. A. Baldis, and J. E. Bernard, "Suppression of Stimulated Raman Scattering by the Seeding of Stimulated Brillouin Scattering in a Laser-Produced Plasma," Phys. Rev. Lett. 59, 1585 (1987). 
57. L. V. Powers and R. L. Berger, "SATIN: A Fluid Code to Study the Nonlinear Evolution of Two-Plasmon Decay and Stimulated Raman Scattering," KMS Fusion, Inc. Report No. KMSF-U1757, 1985.

58. J. Meyer and H. Houtman, "Experimental investigation of the twoplasmon decay instability in a $\mathrm{CO}_{2}$-laser-produced plasma," Phys. Fluids 28, 1549 (1985).

59. D. Russel, D.F. DuBois, and H. Rose, "Enhanced low frequency density fluctuations and resulting $\omega_{0} / 2$ and $\omega_{0}$ radiation from the saturated Two Plasmon Decay instability," presented at the Anomalous Absorption conference, 1994), p.

60. C. J. Walsh, D. M. Villeneuve, and H. A. Baldis, "Electron PlasmaWave Production by Stimulated Raman Scattering: Competition with Stimulated Brillouin Scattering," Phys. Rev. Lett. 53, 1445 (1984).

61. Hugh C. Barr and Francis F. Chen, "Raman scattering in a nearly resonant density ripple," Phys. Fluids 30, 1180 (1987).

62. P. K. Kaw, A. T. Lin, and J. M. Dawson, "Quasiresonant mode coupling of electron plasma waves," Phys. Fluids 16, 1967 (1973). 
63. C. Labaune, H. A. Baldis, S. D. Baton, D. Pesme, and T. Jalinaud, "Upshifted Raman Light produced by Coupling between Stimulated Raman and Brillouin Scattering," Phys. Rev. Lett. 69, 285 (1992).

64. H. C. Barr and F. F. Chen, "SRS with SBS ion waves," Phys. Fluids 30, 1180 (1987).

65. W. Rozmus, A. A. Offenberger, and R. Fedosejevs, "Stimulated Raman backscattering in the presence of ion-acoustic fluctuations," Phys. Fluids 26, 1071 (1983).

66. W. Rozmus, R. P. Sharma, J. C. Samson, and W. Tighe, "Nonlinear evolution of stimulated Raman scattering in homogeneous plasmas," Phys. Fluids 30, 2181 (1987).

67. A. Simon and R. W. Short, "New model of Raman spectra in laserproduced plasma," Phys. Rev. Lett. 53, 1912 (1984).

68. H. Figueroa, C. Joshi, and C. E. Clayton, "Experimental studies of Raman scattering from foam targets using a $0.35 \mu \mathrm{m}$ laser beam," Phys. Fluids $30,586(1987)$.

69. E.J. Turano, C.J. McKinstrie, and W.L. Kruer, "Relativistic Saturation of Forward Stimulated Raman Scattering," presented at the Bull. Am. Phys. Soc, 1994), p. 1754. 
70. Kent Estabrook, W. L. Kruer, and B. F. Lasinski, "Heating by Raman Backscatter and Forward Scatter," Phys. Rev. Lett. 45, 1399 (1980).

71. T. P. Coffey, "Breaking of Large Amplitude Plasma Oscillations," Phys. Fluids 14, 1402 (1971).

72. Kent Estabrook and W. L. Kruer, in Laser Program Annual Report 85 (Lawrence Livermore National Laboratory, Livermore, CA, 1985), pp. 2.

73. Guy Bonnaud, Laser Part. Beams 5, 101 (1987).

74. C.A. Coverdale, C.B. Darrow, C.D. Decker, W.B. Mori, K-C. Tzeng, K.A. Marsh, C.E. Clayton, and C. Joshi, "Propagation of Intense Subpicosecond Laser Pulses through Underdense Plasmas," Phys. Rev. Lett. 74, 4659 (1995).

75. C. S. Liu and P. K. Kaw, "Parametric instabilities in homogeneous unmagnetized plasmas," in Advances in Plasma Physics, edited by A. Simon and W. B. Thompson (Wiley, New York, 1976), pp. 83.

76. W. Seka, B. B. Afeyan, R. Boni, L. M. Goldman, R. W. Short, K. Tanaka, and T. W. Johnston, "Diagnostic value of odd-integer half-harmonic emission from laser-produced plasmas," Phys. Fluids 28, 2570 (1985). 
77. L. M. Goldman, W. Seka, K. Tanaka, R. Short, and A. Simon, "The use of laser harmonic spectroscopy as a target diagnostic," Can. J. Phys. 64, 969 (1986).

78. Bedros B. Afeyan and Edward A. Williams, "Unified Theory of Stimulated Raman Scattering and Two-Plasmon Decay in Inhomogeneous Plasmas: High Frequency Hybrid Instability," Phys. Rev. Lett. 75, 4218 (1995).

79. E. F. Gabl, R. L. Berger, Gar. E. Busch, P. M. Campbell, R. J. Schroeder, C. L. Shepard, and J. A. Tarvin, "Evidence for the modification of half-harmonic spectral shifts by plasma flow," Phys. Fluids B 1, 1850 (1989).

80. R. L. Berger and L. V. Powers, "Emission of light at half-integer harmonics of the laser frequency," Phys. Fluids 28, 2895 (1985).

81. R.W. Means, L. Muschietti, M.Q. Tran, and J. Vaclavik, "Electromagnetic radiation from an inhomogeneous plasma: theory and experiment," Phys. Fluids 24, 2197 (1981).

82. Denise E. Hinkel-Lipsker, Burton D. Fried, and G. J. Morales, "Analytic Expression for Mode Conversion of Langmuir and Electromagnetic Waves," Phys. Rev. Lett. 62, 2680 (1989). 
83. M.C. Richardson, R.S. Craxton, J. Delettrez, R.L. Keck, R.L. McCroy, W. Seka, and J.M. Sources, "Absorption physics at $351 \mathrm{~nm}$ in spherical geometry," Phys. Rev. Lett. 54, 1656 (1985).

84. D. M. Villeneuve, R. L. Keck, B. B. Afeyan, W. Seka, and E. A. Williams, "Production of hot electrons by two-plasmon decay instability in uv laser plasmas," Phys. Fluids 27, 721 (1984).

85. K.L. Baker, "Convective gain in laser produced plasmas," presented at the Anomalous Absorption conference, 1995), p.

86. R.L. Berger (private communication).

87. G. Picard and T. W. Johnston, "Decay instabilities for inhomogeneous plasmas: WKB analysis and absolute instability," Phys. Fluids 28, 859 (1985).

88. John Sheffield, Plasma Scattering of Electromagnetic Radiation (Academic Press, New York, 1975).

89. D. E. Evans and J. Katzenstein, "Laser light scattering in laboratory plasmas," Rep. Prog. Phys. 32, 207 (1969).

90. C. Oberman and G. Auer, "General theory of enhanced induced emission in plasmas," Phys. of Fluids 17, 1980 (1974). 
91. Nicholas A. Krall and Alvin W. Trivelpiece, Principles of Plasma Physics (San Francisco Press, Inc., San Francisco, 1986).

92. I.H. Hutchinson, Principles of plasma diagnotics (Cambridge University Press, 1987).

93. M. N. Rosenbluth and N. Rostoker, "Scattering of electromagnetic waves by a nonequilibrium plasma," Phys. Fluids 5, 776 (1962).

94. H.A. Baldis, N.H. Burnett, and M.C. Richardson, Rev. Sci. Instruments 48, 173 (1977).

95. B. La Fontaine, D.M. Villeneuve, H.A. Baldis, R.P. Drake, and K. Estabrook, "A test of the Landau cutoff of stimulated Raman scattering spectra as an electron-temperature diagnostic in laser-produced plasmas," Phys. Rev. Lett. 68, 484 (1992).

96. A. Simon and R. W. Short, "Energy and Nonlinearity Considerations for the Enhanced Plasma Wave Model of Raman Scattering," Phys. Fluids B 1, 1073 (1989).

97. J. A. Tarvin, Gar. E. Busch, E. F. Gabl, R. J. Schroeder, and C. L. Shepard, "Laser and plasma conditions at the onset of Raman scattering in an underdense plasma," Laser Part. Beams 4, 461 (1986). 
98. W.L. Kruer, "Intense laser plasma interactions: From Janus to Nova," Phys. Fluids B 3, 2356 (1991).

99. W. Seka, E. A. Williams, R. S. Craxton, L. M. Goldman, R. W. Short, and K. Tanaka, "Convective stimulated Raman scattering instability in UV laser plasmas," Phys. Fluids 27, 2181 (1984).

100. R. P. Drake, E. A. Williams, P. E. Young, Kent Estabrook, W. L. Kruer, H. A. Baldis, and T. W. Johnston, "Evidence that Stimulated Raman Scattering in Laser-Produced Plasmas is an Absolute Instability," Phys. Rev. Lett. 60, 1018 (1988).

101. H.C. Barr, T.J.M. Boyd, and A.P. Mackwood, "Stimulated Raman scattering from density cavities in laser-produced plasmas," Phys. Fluids B 4, 2942 (1992).

102. R.P. Drake, "Three-Wave Parametric Instabilities in Long-ScaleLength, Somewhat-Planar, Laser-Produced Plasmas," Las. Part. Beams 10, 599 (1992).

103. D.M. Villeneuve, H.A. Baldis, J.E. Bernard, and R. Benesch, J. Opt. Soc. Am. B 8, 895 (1991). 
104. W. Seka, R.S. Craxton, R.E. Bahr, D.L. Brown, D.K. Bradley, P.A. Jaanimagi, B. Yaakobi, and R. Epstein, "Production and characterization of hot, long-scale-length laser plasmas," Phys. Fluids 4, 432 (1992).

105. R. L. Berger, J. R. Albritton, C. Randall, C. Hanna, W. L. Kruer, A. B. Langdon, and E. A. Williams, "Stopping and Thermalization of Interpenetrating Plasma Streams," Phys. Fluids B 3, (1991).

106. C. S. Liu, M. N. Rosenbluth, and R. B. White, "Parametric Scattering Instabilities in Inhomogeneous Plasma," Phys. Rev. Lett. 31, 697 (1973).

107. C. Labaune, H.A. Baldis, N. Renard, E. Schifano, S.D. Baton, A. Michard, W. Seka, R.E. Bahr, B.S. Bauer, K.L. Baker, and K. Estabrook, "LargeAmplitude Ion Acoustic Waves in a Laser-Produced Plasma," Phys. Rev. Lett. 75, 248 (1995).

108. S. H. Batha, D. D. Meyerhofer, A. Simon, and R. P. Drake, "Raman UpScattering in Long-Scale-Length, Laser-Produced Plasmas," Phys. Fluids B 3, 448 (1991).

109. "The downscattered feature may also include contributions from even number cascades in which further decay of the Langmuir waves driven by LDI reverses the direction of the Langmuir waves.," 
110. E.A. Williams, R.L. Berger, R.P. Drake, A.M. Rubenchik, B.S. Bauer, D.D. Meyerhofer, A.C. Gaeric, and T.W. Johnston, "The frequency and damping of ion acoustic waves in $\mathrm{CH}$ and two-ion-species plasmas," Phys. Plasmas 2, 129 (1995).

111. S. C. Wilks, W. L. Kruer, K. Estabrook, and A. B. Langdon, "Theory and simulation of stimulated Raman scatter at near-forward angles," Phys. Fluids B 4, 2794 (1993).

112. Dwight R. Nicholson and Allan N. Kaufman, "Parametric Instabilities in Turbulent, Inhomogeneous Plasma," Phys. Rev. Lett. 33, 1207 (1974). 